\title{
Revisional and complicated bariatric surgery
}

Citation for published version (APA):

van Wezenbeek, M. R. (2020). Revisional and complicated bariatric surgery. [Doctoral Thesis, Maastricht University]. ProefschriftMaken. https://doi.org/10.26481/dis.20200214mw

Document status and date:

Published: 01/01/2020

DOI:

10.26481/dis.20200214mw

Document Version:

Publisher's PDF, also known as Version of record

\section{Please check the document version of this publication:}

- A submitted manuscript is the version of the article upon submission and before peer-review. There can be important differences between the submitted version and the official published version of record.

People interested in the research are advised to contact the author for the final version of the publication, or visit the DOI to the publisher's website.

- The final author version and the galley proof are versions of the publication after peer review.

- The final published version features the final layout of the paper including the volume, issue and page numbers.

Link to publication

\footnotetext{
General rights rights.

- You may freely distribute the URL identifying the publication in the public portal. please follow below link for the End User Agreement:

www.umlib.nl/taverne-license

Take down policy

If you believe that this document breaches copyright please contact us at:

repository@maastrichtuniversity.nl

providing details and we will investigate your claim.
}

Copyright and moral rights for the publications made accessible in the public portal are retained by the authors and/or other copyright owners and it is a condition of accessing publications that users recognise and abide by the legal requirements associated with these

- Users may download and print one copy of any publication from the public portal for the purpose of private study or research.

- You may not further distribute the material or use it for any profit-making activity or commercial gain

If the publication is distributed under the terms of Article $25 \mathrm{fa}$ of the Dutch Copyright Act, indicated by the "Taverne" license above, 
Revisional and complicated bariatric surgery 


\section{Colofon}

(c) Copyright Martin van Wezenbeek, Maastricht, 2020.

All rights reserved. No part of this book may be reproduced or transmitted in any form or by any means, without prior permission in writing by the author, or when appropriate, by the publishers of the publications.

ISBN: 978-94-6380-686-2

Cover: Huib Dieltjens

Layout: Tiny Wouters

Production: ProefschriftMaken

The research presented in this thesis was conducted at CAPHRI Care and Public Health Research Institute, Department of Epidemiology, of Maastricht University. CAPHRI participates in the Netherlands School of Public Health and Care Research CaRe.

The printing of this thesis was financially supported by Maastricht University \& Catharina Ziekenhuis Eindhoven. 


\title{
Revisional and complicated bariatric surgery
}

\author{
PROEFSCHRIFT \\ ter verkrijging van de graad van doctor aan de Universiteit Maastricht, \\ op gezag van de Rector Magnificus, Prof. Dr. Rianne M. Letschert, \\ volgens het besluit van het College van Decanen, \\ in het openbaar te verdedigen \\ op vrijdag 14 februari 2020 om 14.00 uur
}

door

Martin Richard van Wezenbeek

geboren te Waalwijk op 16 november 1988 


\section{Promotor}

Prof. dr. J.A.W. Teijink

\section{Copromotor}

Dr. S.W. Nienhuijs (Catharina Ziekenhuis)

\section{Beoordelingscommissie}

Prof. dr. N.D. Bouvy (voorzitter)

Dr. F.M.H. van Dielen (Máxima Medisch Centrum)

Prof. dr. J.W. Greve

Prof. dr. E.J. Hazebroek (Wageningen Universiteit)

Prof. dr. A.T.M. Jansen 


\section{CONTENTS}

Chapter 1 General introduction \& outline of the thesis 7

$\begin{array}{lll}\text { PART ONE } & \text { Revisions in bariatric surgery }\end{array}$

Chapter 2 Long-term results of primary vertical banded gastroplasty 25

Chapter $3 \quad$ Medical and psychological predictors for long-term 39 bariatric success using primary vertical banded gastroplasty as a model

Chapter 4 Long-term results after revisions of failed primary vertical banded gastroplasty

Chapter 5 Conventional versus fast track anaesthesia in an unselected group of patients undergoing revisional bariatric surgery

Chapter 6 Conversion to gastric bypass after either failed gastric band 83 or failed sleeve gastrectomy

Chapter 7 Matched short-term results of SADI versus GBP after sleeve gastrectomy

Chapter 8 Single Anastomosis duodenoileal bypass or GAstric bypass after primary sleeve gastrectomy (SAGA-trial): a randomized controlled trial (Study protocol)

PART TWO Early complications in bariatric surgery

Chapter 9 Predictors for the occurrence of major complications 129 after primary Roux-en-Y Gastric Bypass surgery

Chapter 10 Transection versus preservation of the neurovascular 143 bundle of the lesser omentum in primary Roux-en$\mathrm{Y}$ gastric bypass surgery

Chapter 11 A specifically designed stent for anastomotic leaks after 159 bariatric surgery: Experiences in a tertiary referral hospital 
Valorisation addendum 197

Dankwoord 203

List of publications 209

Curriculum Vitae 213 


\section{CHAPTER 1}

General introduction \& outline of the thesis 



\section{GENERAL INTRODUCTION}

\section{Focus on revisions and complications}

The consequences of the global obesity pandemic are enormous. They range from associated chronic diseases to premature deaths with a high impact on quality of life, health resources and costs. Treatment of obesity is challenging since it is a complex condition with serious social and psychological dimensions, which affects all ages and socioeconomic groups. Of the available options, a surgical intervention is the only long term effective treatment. ${ }^{1}$ The transition towards laparoscopic surgery, concentrating care in high volume centers and improvements in anesthesiological approach have all attributed to the acceptance of bariatric surgery as a safe option. Even though it is the best available option in the treatment of (morbid) obesity, bariatric surgery remains associated with complications. Leakage and postoperative bleeding are shortterm examples, dysphagia or weight regain necessitating a revisional procedure can occur on the long scale. The biggest challenge is to further reduce the risk of these adverse events. For this purpose, this manuscript focuses on these short- and long-term complications.

\section{Obesity: Definition, epidemiology and etiology}

Overweight and obesity are caused by an abnormal or excessive fat accumulation which impairs general health. Body Mass Index (BMI) is the most commonly used classification. It is a simple index that defines a person's weight in kilograms divided by the square of the height in meters $\left(\mathrm{kg} / \mathrm{m}^{2}\right)$. Overweight is defined as a BMl greater than or equal to $25 \mathrm{~kg} / \mathrm{m}^{2}$ and obesity describes a $\mathrm{BMl}$ greater than or equal to $30 \mathrm{~kg} / \mathrm{m}^{2}$.

Obesity is an often preventable medical condition which increases the risk at developing various morbidities such as cardiovascular diseases, diabetes mellitus type 2, obstructive sleep apnea, depression, osteoarthritis and certain types of cancer. ${ }^{3,4}$ Furthermore, it is one of the leading preventable causes of death worldwide. ${ }^{5}$

Between 1975 and 2016, the incidence of worldwide obesity has nearly tripled. In 2016, $13 \%$ of the global adult population was obese, adding up to an astonishing 650 million obese adults worldwide. ${ }^{2}$ Furthermore, in 2016, another 340 million children and adolescents between the age of 5 and 19 years old were either overweight or obese. ${ }^{2}$ These numbers highlight the fact that obesity is a global burden which has been expanding over the last years. Consequently, this will greatly affect the health care costs both now as well as in the future. ${ }^{6,7}$ 
According to the definitions of the World Health Organization, Caucasian people are considered obese when their Body Mass Index (BMI) is over 30 $\mathrm{kg} / \mathrm{m}^{2}$. Morbid obesity is considered in case of a BMI $\geq 40 \mathrm{~kg} / \mathrm{m}^{2}$ or a BMI $\geq 35$ $\mathrm{kg} / \mathrm{m}^{2}$ while experiencing obesity-related health conditions. These conditions include diabetes mellitus type 2, hypertension, dyslipidemia, obstructive sleep apnea syndrome and osteoarthritis.

Major causes for obesity are excessive food intake and a lack of physical activity, making obesity a largely preventable disease through a combination of personal choices and social changes. ${ }^{2}$ The high number of obese patients within a community with a low societal level may be caused by the low cost of energy-dense food. This hypothesis is likely enforced by the high palatability of sugar and fat. ${ }^{8}$ Only a minor part of the obese population has a genetic, medical or psychiatric illness as the primary cause for their obesity. ${ }^{9}$

The brain-gut axis has a major role in the control of food intake. During an empty stomach, the "hunger hormone" ghrelin is excreted to increase hunger by stimulation of the hypothalamus. This effect stops when the stomach wall is stretched, inhibiting the secretion of ghrelin. ${ }^{10}$ Other hormones such as leptin, peptide-YY, glucagon-like peptide and cholecystokinin also affect the central nervous system to regulate hunger and satiety and to control food intake.

Many contributing factors result in overweight and even obesity. These factors include genetic, behavioral, environmental, hormonal, social and cultural factors, which result in an energy imbalance and promote excessive fat deposition. ${ }^{11}$ Studies show that behavioral and environmental factors play a predominant role in the current obesity epidemic. ${ }^{12}$ In order to prevent weight regain, a common complication after bariatric surgery, it is of vital importance for patients to establish the necessary lifestyle changes.

\section{Bariatric procedures}

Bariatric surgery started in the 1950's with an intestinal bypass by laparotomy. Malabsorption of food was established by diverting a large portion of the small intestine, causing weight loss. Over the last decades, a number of different procedures have been developed to achieve even more weight loss. Figure 1.1 shows some of the highlights of the history of bariatric surgery. The procedures relevant to this manuscript will be reviewed below. 


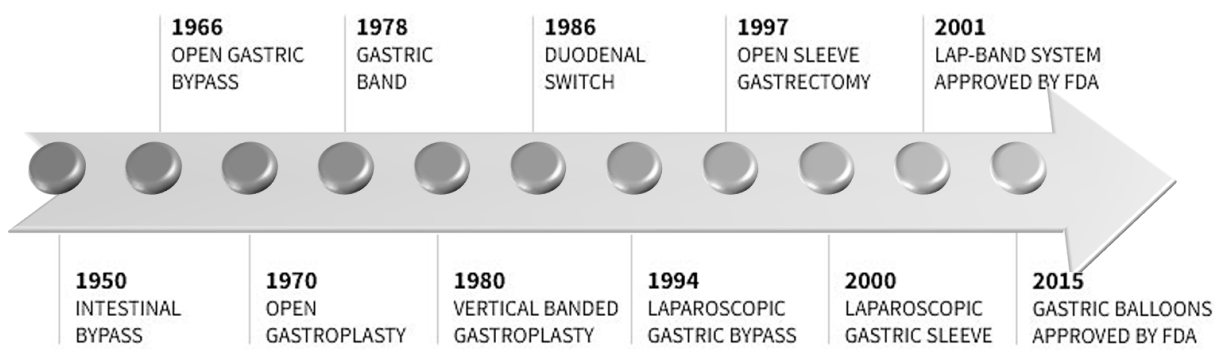

Figure 1.1 Important landmarks in the history of bariatric surgery.

\subsection{Roux-en-Y gastric bypass (RYGB)}

The original gastric bypass was introduced by dr. Mason in $1966 .{ }^{13}$ The first gastric bypass consisted of a small portion of the stomach along with a retrocolic loop gastrojejunostomy. Over the last decades, this procedure was further developed. Nowadays, the most performed type of gastric bypass is the Roux-en-Y gastric bypass. This procedure consists of a small gastric pouch from the upper stomach and a reconstruction of the gastro-intestinal tract to enable drainage of both gastric segments. Reconstruction of the intestinal tract is performed in many varieties, resulting in different lengths of the small intestine segments. There are three limbs: the alimentary limb (also known as the Roux limb; between the gastric pouch and the jejuno-jejunostomy), the biliopancreatic limb (the bypassed section including the stomach remnant, the duodenum and a portion of the proximal jejunum up to the jejunojejunostomy) and the common limb (originating distally from the jejunojejunostomy).

There are many varieties on the gastric bypass, such as the more recently introduced mini or omegaloop gastric bypass and the duodenojejunal bypass. Currently, RYGB is still considered the gold standard in bariatric surgery with good long-term results with a mean weight loss over $60 \%$, a significant reduction of obesity-related comorbidities, a low revision rate and a high patient satisfaction rate. ${ }^{14}$

\subsection{Adjustable Gastric Band (AGB)}

The first gastric band was placed by Dr. Wilkinson, who aimed to achieve weight loss without disrupting the gastro-intestinal tract. His main goal was a restriction in food intake. The first band, a non-adjustable $2 \mathrm{~cm}$ Marlex mesh, was placed in $1978 .{ }^{15}$ Later, in the mid 1980 's, both Kuzmak and Hallberg separately developed the more modern version to the band. This was a silicone band which allowed for adjustments by the use of a subcutaneous port to 
ensure comfort and effectiveness of the band. The band can be filled with a saline solution until the optimal restriction is achieved. ${ }^{16}$ Average long-term weight loss is reported to be close to $50 \%$ of all excess weight. ${ }^{17}$ In 2008 , over $40 \%$ of all bariatric procedures consisted of a gastric band, however these percentages have quickly decreased over the last years. ${ }^{18,19}$

\subsection{Vertical Banded Gastroplasty (VBG)}

This old restrictive procedure was first described by dr. Edward Mason in $1980 .^{20}$ In a VBG, a small gastric pouch is created by vertical stapling of the fundus and placing a band at the distal end of the pouch. The biggest downfall of this procedure was a high amount of gastro-gastric fistulae due to a high percentage of staple-line disruptions. Therefore, MacLean later altered the procedure by dividing this staple line. ${ }^{21,22}$

Weight loss was obtained by restricting food intake due to a small gastric pouch. This in turn established early satiety while maintaining normal passage through the intestines, avoiding any malabsorption of nutrients and medication.

In the early days, VBG has proven to provide good short-term weight loss results and a low complication rate. ${ }^{23-26}$

\subsection{Sleeve gastrectomy}

A more modern restrictive procedure is the sleeve gastrectomy. It was first described by Marceau et al. in the late 1990's as part of a biliopancreatic diversion with duodenal switch (BPD/DS) and was later popularized by Gagner et al. as standalone procedure. ${ }^{27-29}$

This procedure reduces the stomach size to about $15-20 \%$ of its original size by surgically removing a large portion of the stomach along the greater curvature, the predominant area for the production of ghrelin, the hunger regulating peptide hormone. 30,31

Adequate long-term results are scarce, but SG is showing promising results in terms of weight loss and improvement of obesity related comorbidities in the short- and mid-term follow-up. ${ }^{32,33}$ Furthermore, it has quickly become one the most performed procedures worldwide. ${ }^{19}$

\subsection{Single Anastomosis Duodenolleal bypass (SADI)}

One of the newest malabsorptive procedures which is quickly gaining in popularity is the single anastomosis duodenoileal bypass. It was first introduced by Torres et al. in 2007. ${ }^{34}$ The SADI is a derivative of the primary procedure SADI-S (SADI with sleeve gastrectomy), which can be described as a simplified duodenal switch and has demonstrated to have satisfactory short- and 
medium-term results. ${ }^{35}$ Furthermore, it may be a valuable alternative to the technically more challenging biliopancreatic diversion. Even though the SADI appears to be a valid option since the first reported results are promising, more data is necessary to fully comprehend this new procedure. ${ }^{34-37}$ In 2018, the International Federation for the Surgery of Obesity and Metabolic Disorders (IFSO), has marked SADI(-S) as a recognized bariatric procedure, but underlines the lack of long-term results and its potential unforeseeable long-term consequences such as nutrient deficiencies. ${ }^{38}$

Important note:

As mentioned earlier, obesity is caused by a large range of factors. Proper treatment of the predominant behavioral and environmental risk factors does not only require a bariatric procedure, but also demands proper screening, preeducation and postoperative follow-up by a dedicated bariatric team including dieticians, psychologists, physical therapists, surgeons, internists, gastroenterologists and specialized nurses.

\section{Benefits}

An important benchmark in research on the results of bariatric surgery is the Swedish Obese Subjects (SOS) Registry, a large, long-term, prospective study comparing several bariatric procedures with the usual (conservative) treatment reporting on a wide range of outcome parameters.

Important results were an average long-term (total) weight loss between 16 and $23 \%$ with a reduction in the overall mortality rate (hazard ratio $=0.71$ ) in favor of bariatric surgery. Furthermore, bariatric surgery establishes favorable rates of recovery from diabetes, hypertriglyceridemia, low levels of high-density lipoprotein cholesterol, hypertension, and hyperuricemia. Finally, a reduction in cancer incidence was observed in female study subjects after surgery. ${ }^{1,39-41}$

Other studies report an improvement of health-related quality of life and other psychological parameters. $^{42-44}$

\section{Complications in bariatric surgery and its provoking factors}

Even though bariatric surgery appears to be largely beneficial, one of the biggest challenges is to prevent and limit any type of complication as much as possible. The term complication is a very broad conception, due to the large amount of different causes and the word complication can be interpreted in more than one way. 


\subsection{Psychological factors}

Some of the factors that may affect the outcome after bariatric surgery are personal (social and psychological) patient factors and the commitment to make the necessary lifestyle changes after surgery. Important factors, such as eating behavior, socio-economic status and coping are known to affect these changes, thus affecting the outcome after bariatric surgery. ${ }^{45-47}$ In these cases, failure is often considered when an insufficient amount of weight loss is observed at the long-term.

The simple solution would be to identify these various factors and properly tackle them before and after surgery to ensure a good long-term result. This identification is impeded by the great diversity in results, outcome measures, diverse lengths of follow-up and small patient groups. ${ }^{46}$ This in turn leads to a lack of consensus on which factors have a predictive value on the outcome after bariatric surgery. ${ }^{48}$

\subsection{In-hospital care}

Obesity and its related anatomical changes create challenges for clinicians, as bariatric patients requires a different but still appropriate selection and dosing of medications for pain management and treatment of infectious complications, venous thromboembolisms, bleeding and other chronic diseases. ${ }^{49}$ Inappropriate treatment of these obese patients may potentially lead to a different kind of failure: an increase in peri-operative complications, such as bleeding, anastomotic leakage, wound infection, venous thromboembolisms and cardiovascular events leading to increased mortality and readmission rate. ${ }^{50}$

The annual number of performed bariatric procedures worldwide is currently over 500,000 and is still growing. ${ }^{51}$ Even though the complication rate for these type of procedures is relatively low, the high number of performed procedures still results in a high absolute number of complications. Due to these large numbers, associated costs will certainly impact health care economy. ${ }^{6,7}$ This calls for continuous efforts and research to reduce the number of complications. There are different areas to focus on, such as identifying risk factors associated with a higher postoperative complication rate. ${ }^{52}$ Even different intra-operative surgical techniques may affect the outcome. For example: usage of the pars flaccida technique versus the perigastric technique in AGB-placement or the choice of bougie caliber in sleeve gastrectomy. ${ }^{53,54}$ Nonetheless, it is unlikely that complications such as bleeding, anastomotic leakage and intra-abdominal abscesses are completely avoidable, which calls for a highly reliable treatment option. However, therapeutic options are diverse and can cause complications on their own. ${ }^{49,55,56}$ 
With the continuous growing number of bariatric procedures, fast track protocols in hospitals have become more attractive, which in theory could have led to a negative effect on the perioperative outcome after bariatric surgery. Fortunately, fast track protocols have proven to be safe and effective in primary bariatric surgery. ${ }^{57,58}$ However, evidence on its positive effect in patients undergoing more challenging and less common revisional bariatric surgery is still very scarce. This seems relevant since the number of revisional procedures is expected to increase over the next years.

\subsection{Type of procedure}

Failure depends on many factors among which the type of procedure is important. For example: literature is increasingly showing that older (restrictive) procedures such as the $A G B$ and the VBG are often not living up to expectations. Furthermore, the percentage of complications requiring revisional surgery is unacceptably high after VBG and $A G B .^{59-61}$ Recent literature reports a poor long-term outcome, mainly focusing on a variety of potential technical problems, weight loss and biomedical parameters. ${ }^{59,62,63}$ For these reasons, both procedures have been (largely) abandoned in the Netherlands for several years in favor of other bariatric techniques. ${ }^{64}$

As mentioned before, sleeve gastrectomy appears to be a promising alternative to the older restrictive procedures. However, one of the major drawbacks of the sleeve gastrectomy is similar to the older procedures: development of complaints necessitating a revisional procedure (including insufficient weight loss and complaints of gastro-esophageal reflux disease). Proper long-term data is still scarce but the currently reported revision rates are lower than both the $A G B$ and $V B G .{ }^{65,66} A$ recent study from Chang et al. reports a revision rate of almost $17 \%{ }^{67}$

\subsection{Revisional surgery}

The most common option for revisional surgery after either failed $A B G$, VBG or sleeve gastrectomy is the RYGB, with a satisfactory outcome in terms of weight loss, morbidity and reduction in obesity-related comorbidities. ${ }^{68-70}$ Nonetheless, the first results of a revisional gastric bypass after sleeve gastrectomy are often disappointing in terms of (additional) weight loss. The failure of this type of revisional surgery has led to the search for a better alternative. ${ }^{71}$ Current alternatives are the biliopancreatic diversion, the duodenal switch and the more recently introduced single anastomosis duodeno-ileal bypass (SADI). ${ }^{36,72,73}$ Data on the outcome after SADI, especially as a revisional procedure, as well as comparison with other procedures is scarce. 


\section{OUTLINE OF THE THESIS}

Different types of failure and complications will be highlighted in two main themes: revisional surgery and early postoperative complications. This thesis aims to contribute to the reduction of short- and long-term complications by analyzing current results of revisional surgery, finding predictors for failure and complications and assessing the effect of intra-operative techniques.

\section{Part one}

The first part of this thesis focuses on revisional bariatric surgery and its difficulties. Revisional procedures are used to treat several long-term complications such as weight regain, insufficient weight loss, gastro-esophageal reflux disease and dysphagia.

To fully understand the indications for revisional surgery, a proper understanding of the primary (failed) procedure is essential. Therefore, Chapter 2 focuses on the long-term medical outcome after primary VBG and its associated complications. To better understand the reason for these failures, a search for potential psychological and medical factors associated with failure has been conducted. The results will be discussed in Chapter 3.

When is revisional surgery indicated after failed VBG, what type of surgery provides the best (long-term) outcome? Chapter 4 attempts to answer these questions. Different revisional procedures after primary failed VBG are analyzed, including revision of the VBG, conversion to sleeve gastrectomy and conversion to RYGB.

As the number of revisional procedures is expected to rise over the next years, Chapter 5 evaluates the use of a fast track protocol in bariatric revisional surgery, which may increase the number of (revisional) procedures performed on a daily basis without an increase in early postoperative complications.

The thesis continues with Chapter 6, focusing on the conversion of both a failed AGB and a failed sleeve gastrectomy to RYGB. Current literature suggests RYGB is feasible as revisional procedure after almost every type of failed (primary) procedure. The comparison in Chapter 6 is made to evaluate whether this assumption is valid.

As mentioned before, new surgical techniques are arising. The first published results on the SADI are promising, however, much is unknown about this new procedure. Chapter 7 focuses on the use of SADI as a revisional procedure after primary sleeve gastrectomy to potentially find a better alternative to the RYGB. To further investigate the effects of the SADI as a revisional procedure, Chapter 8 describes a study protocol for a randomized controlled trial analyzing the outcome of SADI versus RYGB after sleeve gastrectomy. 


\section{Part two}

The second part of the thesis focuses on early postoperative complications in bariatric surgery. Different aspects are reviewed. Chapter 9 focuses on the identification of potential risk factors leading to postoperative complications after primary RYGB. Chapter 10 assesses a technique to reduce the number of postoperative complications in primary RYGB. The final chapter of part two, Chapter 11, focuses on a new (endoscopic) treatment option for one of the most difficultly manageable complications: anastomotic leakage.

The thesis will be ended by a summary of the results with a general discussion and future perspectives. 


\section{REFERENCES}

1. Sjostrom L, Lindroos AK, Peltonen M, Torgerson J, Bouchard C, Carlsson B, et al. Lifestyle, diabetes, and cardiovascular risk factors 10 years after bariatric surgery. $\mathrm{N}$ Engl J Med. 2004;351(26):2683-93.

2. WHO. Obesity and overweight fact sheet 2017 [updated October 2017; cited 2017 16-102017]. Available from: http://www.who.int/mediacentre/factsheets/fs311/en/.

3. Haslam DW, James WP. Obesity. Lancet. 2005;366(9492):1197-209.

4. Luppino FS, de Wit LM, Bouvy PF, Stijnen T, Cuijpers P, Penninx BW, et al. Overweight, obesity, and depression: a systematic review and meta-analysis of longitudinal studies. Arch Gen Psychiatry. 2010;67(3):220-9.

5. Allison $\mathrm{DB}$, Fontaine $\mathrm{KR}$, Manson JE, Stevens J, Vanltallie TB. Annual deaths attributable to obesity in the United States. JAMA. 1999;282(16):1530-8.

6. Cawley J, Meyerhoefer C. The medical care costs of obesity: an instrumental variables approach. J Health Econ. 2012;31(1):219-30.

7. Kim DD, Basu A. Estimating the Medical Care Costs of Obesity in the United States: Systematic Review, Meta-Analysis, and Empirical Analysis. Value Health. 2016;19(5):602-13.

8. Drewnowski A, Specter SE. Poverty and obesity: the role of energy density and energy costs. Am J Clin Nutr. 2004;79(1):6-16.

9. Bleich S, Cutler D, Murray C, Adams A. Why is the developed world obese? Annu Rev Public Health. 2008;29:273-95.

10. Geary N. Endocrine controls of eating: CCK, leptin, and ghrelin. Physiol Behav. 2004;81(5): 719-33.

11. Racette SB, Deusinger SS, Deusinger RH. Obesity: overview of prevalence, etiology, and treatment. Phys Ther. 2003;83(3):276-88.

12. Hill JO, Peters JC. Environmental contributions to the obesity epidemic. Science. 1998;280(5368): 1371-4.

13. Mason EE, Ito C. Gastric bypass in obesity. Surg Clin North Am. 1967;47(6):1345-51.

14. Edholm D, Svensson F, Naslund I, Karlsson FA, Rask E, Sundbom M. Long-term results 11 years after primary gastric bypass in 384 patients. Surg Obes Relat Dis. 2013;9(5):708-13.

15. Wilkinson LH, Peloso OA. Gastric (reservoir) reduction for morbid obesity. Arch Surg. 1981;116(5):602-5.

16. Steffen R. The history and role of gastric banding. Surg Obes Relat Dis. 2008;4(3 Suppl):S7-13.

17. Maggard MA, Shugarman LR, Suttorp $M$, Maglione $M$, Sugerman $H J$, Livingston $E H$, et al. Meta-analysis: surgical treatment of obesity. Ann Intern Med. 2005;142(7):547-59.

18. Buchwald H, Oien DM. Metabolic/bariatric surgery Worldwide 2008. Obes Surg. 2009;19(12):1605-11.

19. Angrisani L, Santonicola A, lovino P, Formisano G, Buchwald H, Scopinaro N. Bariatric Surgery Worldwide 2013. Obes Surg. 2015;25(10):1822-32.

20. Mason EE. Vertical banded gastroplasty for obesity. Arch Surg. 1982;117(5):701-6.

21. MacLean LD, Rhode BM, Forse RA. Late results of vertical banded gastroplasty for morbid and super obesity. Surgery. 1990;107(1):20-7.

22. MacLean LD, Rhode BM, Forse RA. A gastroplasty that avoids stapling in continuity. Surgery. 1993;113(4):380-8.

23. Buchwald $H$, Avidor $Y$, Braunwald E, Jensen MD, Pories W, Fahrbach $K$, et al. Bariatric surgery: a systematic review and meta-analysis. JAMA. 2004;292(14):1724-37.

24. Lin YC, Chou FF, Chen SM, Wu CH. Vertical banded gastroplasty: a simple, effective and safe surgery for morbid obesity. Chang Gung Med J. 2003;26(10):754-60.

25. Miller K, Pump A, Hell E. Vertical banded gastroplasty versus adjustable gastric banding: prospective long-term follow-up study. Surg Obes Relat Dis. 2007;3(1):84-90.

26. Morino M, Toppino M, Bonnet G, Rosa R, Garrone C. Laparoscopic vertical banded gastroplasty for morbid obesity. Assessment of efficacy. Surg Endosc. 2002;16(11):1566-72. 
27. Gumbs AA, Gagner M, Dakin G, Pomp A. Sleeve gastrectomy for morbid obesity. Obes Surg. 2007;17(7):962-9.

28. Marceau P, Hould FS, Simard S, Lebel S, Bourque RA, Potvin M, et al. Biliopancreatic diversion with duodenal switch. World J Surg. 1998;22(9):947-54.

29. Regan JP, Inabnet WB, Gagner M, Pomp A. Early experience with two-stage laparoscopic Roux-en-Y gastric bypass as an alternative in the super-super obese patient. Obes Surg. 2003;13(6):861-4.

30. Langer FB, Reza Hoda MA, Bohdjalian A, Felberbauer FX, Zacherl J, Wenzl E, et al. Sleeve gastrectomy and gastric banding: effects on plasma ghrelin levels. Obes Surg. 2005;15(7):1024-9.

31. Masuda $\mathrm{Y}$, Tanaka T, Inomata N, Ohnuma N, Tanaka S, Itoh Z, et al. Ghrelin stimulates gastric acid secretion and motility in rats. Biochem Biophys Res Commun. 2000;276(3):905-8.

32. van Rutte PW, Smulders JF, de Zoete JP, Nienhuijs SW. Outcome of sleeve gastrectomy as a primary bariatric procedure. Br J Surg. 2014;101(6):661-8.

33. Vidal P, Ramon JM, Goday A, Benaiges D, Trillo L, Parri A, et al. Laparoscopic gastric bypass versus laparoscopic sleeve gastrectomy as a definitive surgical procedure for morbid obesity. Mid-term results. Obes Surg. 2013;23(3):292-9.

34. Sanchez-Pernaute A, Herrera MA, Perez-Aguirre ME, Talavera P, Cabrerizo L, Matia P, et al. Single anastomosis duodeno-ileal bypass with sleeve gastrectomy (SADI-S). One to three-year follow-up. Obes Surg. 2010;20(12):1720-6.

35. Sanchez-Pernaute A, Rubio MA, Perez Aguirre E, Barabash A, Cabrerizo L, Torres A. Singleanastomosis duodenoileal bypass with sleeve gastrectomy: metabolic improvement and weight loss in first 100 patients. Surg Obes Relat Dis. 2013;9(5):731-5.

36. Sanchez-Pernaute A, Rubio MA, Conde M, Arrue E, Perez-Aguirre E, Torres A. Singleanastomosis duodenoileal bypass as a second step after sleeve gastrectomy. Surg Obes Relat Dis. 2015;11(2):351-5.

37. Sanchez-Pernaute A, Rubio MA, Cabrerizo L, Ramos-Levi A, Perez-Aguirre E, Torres A. Singleanastomosis duodenoileal bypass with sleeve gastrectomy (SADI-S) for obese diabetic patients. Surg Obes Relat Dis. 2015;11(5):1092-8.

38. Brown WA, Ooi G, Higa K, Himpens J, Torres A, SADI-S/OADS IF-atfrtlo. Single Anastomosis Duodenal-Ileal Bypass with Sleeve Gastrectomy/One Anastomosis Duodenal Switch (SADIS/OADS) IFSO Position Statement. Obes Surg. 2018;28(5):1207-16.

39. Sjostrom L. Review of the key results from the Swedish Obese Subjects (SOS) trial - a prospective controlled intervention study of bariatric surgery. J Intern Med. 2013;273(3): 219-34.

40. Sjostrom L, Gummesson A, Sjostrom CD, Narbro K, Peltonen M, Wedel H, et al. Effects of bariatric surgery on cancer incidence in obese patients in Sweden (Swedish Obese Subjects Study): a prospective, controlled intervention trial. Lancet Oncol. 2009;10(7):653-62.

41. Sjostrom L, Narbro K, Sjostrom CD, Karason K, Larsson B, Wedel H, et al. Effects of bariatric surgery on mortality in Swedish obese subjects. N Engl J Med. 2007;357(8):741-52.

42. van Hout GC, Fortuin FA, Pelle AJ, Blokland-Koomen ME, van Heck GL. Health-related quality of life following vertical banded gastroplasty. Surg Endosc. 2009;23(3):550-6.

43. van Hout GC, Fortuin FA, Pelle AJ, van Heck GL. Psychosocial functioning, personality, and body image following vertical banded gastroplasty. Obes Surg. 2008;18(1):115-20.

44. Herpertz S, Muller A, Burgmer R, Crosby RD, de Zwaan M, Legenbauer T. Health-related quality of life and psychological functioning 9 years after restrictive surgical treatment for obesity. Surg Obes Relat Dis. 2015;11(6):1361-70.

45. Hsu LK, Benotti PN, Dwyer J, Roberts SB, Saltzman E, Shikora S, et al. Nonsurgical factors that influence the outcome of bariatric surgery: a review. Psychosom Med. 1998;60(3):338-46.

46. van Hout GC, Hagendoren CA, Verschure SK, van Heck GL. Psychosocial predictors of success after vertical banded gastroplasty. Obes Surg. 2009;19(6):701-7. 
47. Mathus-Vliegen EM. Long-term health and psychosocial outcomes from surgically induced weight loss: results obtained in patients not attending protocolled follow-up visits. Int J Obes (Lond). 2007;31(2): 299-307.

48. Waumsley A, Atter N, Boyle S, et a. Obesity Working Group 2011: Obesity in the UK: A psychological perspective. In: Society TBP, editor. 2011.

49. Quidley AM, Bland CM, Bookstaver PB, Kuper K. Perioperative management of bariatric surgery patients. Am J Health Syst Pharm. 2014;71(15):1253-64.

50. Daigle CR, Brethauer SA, Tu C, Petrick AT, Morton JM, Schauer PR, et al. Which postoperative complications matter most after bariatric surgery? Prioritizing quality improvement efforts to improve national outcomes. Surg Obes Relat Dis. 2018;14(5):652-7.

51. Angrisani L, Santonicola A, lovino P, Vitiello A, Zundel N, Buchwald H, et al. Bariatric Surgery and Endoluminal Procedures: IFSO Worldwide Survey 2014. Obes Surg. 2017.

52. Finks JF, Kole KL, Yenumula PR, English WJ, Krause KR, Carlin AM, et al. Predicting risk for serious complications with bariatric surgery: results from the Michigan Bariatric Surgery Collaborative. Ann Surg. 2011;254(4):633-40.

53. Di Lorenzo N, Furbetta F, Favretti F, Segato G, De Luca M, Micheletto G, et al. Laparoscopic adjustable gastric banding via pars flaccida versus perigastric positioning: technique, complications, and results in 2,549 patients. Surg Endosc. 2010;24(7):1519-23.

54. Yuval JB, Mintz Y, Cohen MJ, Rivkind Al, Elazary R. The effects of bougie caliber on leaks and excess weight loss following laparoscopic sleeve gastrectomy. Is there an ideal bougie size? Obes Surg. 2013;23(10):1685-91.

55. Spieker H, Dietrich A. [Bleeding complications in bariatric surgery: Prophylaxis and therapy]. Chirurg. 2015;86(9):833-40.

56. Jacobsen HJ, Nergard BJ, Leifsson BG, Frederiksen SG, Agajahni E, Ekelund M, et al. Management of suspected anastomotic leak after bariatric laparoscopic Roux-en-y gastric bypass. Br J Surg. 2014;101(4):417-23.

57. Dogan K, Kraaij L, Aarts EO, Koehestanie P, Hammink E, van Laarhoven CJ, et al. Fast-track bariatric surgery improves perioperative care and logistics compared to conventional care. Obes Surg. 2015; 25(1):28-35.

58. Geubbels N, Bruin SC, Acherman Yl, van de Laar AW, Hoen MB, de Brauw LM. Fast track care for gastric bypass patients decreases length of stay without increasing complications in an unselected patient cohort. Obes Surg. 2014;24(3):390-6.

59. O'Brien PE, MacDonald L, Anderson M, Brennan L, Brown WA. Long-term outcomes after bariatric surgery: fifteen-year follow-up of adjustable gastric banding and a systematic review of the bariatric surgical literature. Ann Surg. 2013;257(1):87-94.

60. Suter M, Calmes JM, Paroz A, Giusti V. A 10-year experience with laparoscopic gastric banding for morbid obesity: high long-term complication and failure rates. Obes Surg. 2006;16(7): 829-35.

61. Vinzens F, Kilchenmann A, Zumstein V, Slawik M, Gebhart M, Peterli R. Long-term outcome of laparoscopic adjustable gastric banding (LAGB): results of a Swiss single-center study of 405 patients with up to 18 years' follow-up. Surg Obes Relat Dis. 2017;13(8):1313-9.

62. Jennings NA, Boyle M, Mahawar K, Balupuri S, Small PK. Revisional laparoscopic Roux-en-Y gastric bypass following failed laparoscopic adjustable gastric banding. Obes Surg. 2013;23(7):947-52.

63. Ngiam KY, Khoo VY, Kong L, Cheng AK. Laparoscopic Adjustable Gastric Banding Revisions in Singapore: a 10-Year Experience. Obes Surg. 2016;26(5):1069-74.

64. Schouten R, Wiryasaputra DC, van Dielen FM, van Gemert WG, Greve JW. Long-term results of bariatric restrictive procedures: a prospective study. Obes Surg. 2010;20(12):1617-26.

65. Gautier T, Sarcher T, Contival N, Le Roux Y, Alves A. Indications and mid-term results of conversion from sleeve gastrectomy to Roux-en-Y gastric bypass. Obes Surg. 2013;23(2): 212-5. 
66. van Rutte PW, Smulders JF, de Zoete JP, Nienhuijs SW. Indications and short-term outcomes of revisional surgery after failed or complicated sleeve gastrectomy. Obes Surg. 2012;22(12):1903-8.

67. Chang DM, Lee WJ, Chen JC, Ser KH, Tsai PL, Lee YC. Thirteen-Year Experience of Laparoscopic Sleeve Gastrectomy: Surgical Risk, Weight Loss, and Revision Procedures. Obes Surg. 2018;28(10):2991-7.

68. Angrisani L, Vitiello A, Santonicola A, Hasani A, De Luca M, lovino P. Roux-en-Y Gastric Bypass Versus Sleeve Gastrectomy as Revisional Procedures after Adjustable Gastric Band: 5-Year Outcomes. Obes Surg. 2017;27(6):1430-7.

69. Malinka T, Zerkowski J, Katharina I, Borbely YM, Nett P, Kroll D. Three-Year Outcomes of Revisional Laparoscopic Gastric Bypass after Failed Laparoscopic Sleeve Gastrectomy: a CaseMatched Analysis. Obes Surg. 2017;27(9):2324-30.

70. Vasas $P$, Dillemans B, Van Cauwenberge $S$, De Visschere $M$, Vercauteren $C$. Short- and longterm outcomes of vertical banded gastroplasty converted to Roux-en-Y gastric bypass. Obes Surg. 2013; 23(2):241-8.

71. Parmar CD, Mahawar KK, Boyle M, Schroeder N, Balupuri S, Small PK. Conversion of Sleeve Gastrectomy to Roux-en-Y Gastric Bypass is Effective for Gastro-Oesophageal Reflux Disease but not for Further Weight Loss. Obes Surg. 2017;27(7):1651-8.

72. Carmeli I, Golomb I, Sadot E, Kashtan H, Keidar A. Laparoscopic conversion of sleeve gastrectomy to a biliopancreatic diversion with duodenal switch or a Roux-en-Y gastric bypass due to weight loss failure: our algorithm. Surg Obes Relat Dis. 2015;11(1):79-85.

73. Homan J, Betzel B, Aarts EO, van Laarhoven KJ, Janssen IM, Berends FJ. Secondary surgery after sleeve gastrectomy: Roux-en-Y gastric bypass or biliopancreatic diversion with duodenal switch. Surg Obes Relat Dis. 2015;11(4):771-7. 



\section{PART ONE}

Revisions in bariatric surgery 



\section{CHAPTER}

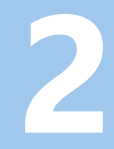

Long-term results of primary vertical banded

gastroplasty

M.R. van Wezenbeek

J.F. Smulders

J.P.J.G.M. de Zoete

M.D. Luyer

G. van Montfort

S.W. Nienhuijs

Obesity Surgery. 2015;25(8):1425-30

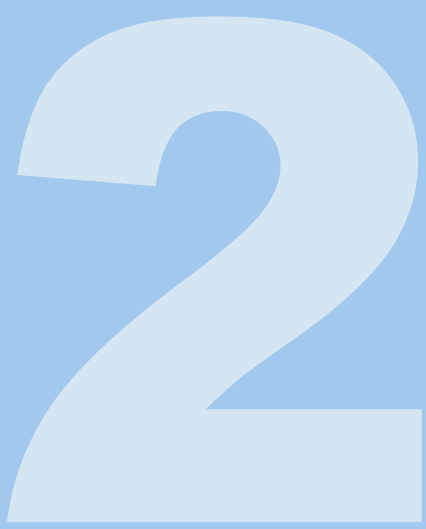




\section{ABSTRACT}

\section{Background}

The vertical banded gastroplasty (VBG) used to be a common restrictive bariatric procedure, but has been abandoned by many due to a high failurerate, a high incidence of long-term complications and the newer adjustable gastric band (AGB) and sleeve. However, potential favourable long-term results and the upcoming banded gastric bypass, with a similar mechanical outlet restriction and control of the pouch size, renewed our interest in the VBG. Therefore we investigated the long-term outcome of primary VBG at the Catharina hospital in the Netherlands.

\section{Materials and methods}

Patients that underwent a primary VBG between 1998 and 2008 were included. Patients' characteristics, operative details, evolution on weight and comorbidities, complications and outcome of revisions were reviewed.

\section{Results}

A total of 392 patients ( $80 \%$ female) were reviewed with a mean age of $40 \pm 9$ years and Body Mass Index of $44 \pm 5 \mathrm{~kg} / \mathrm{m}^{2}$. Mean follow-up after VBG was $66 \pm 50$ months and showed a mean excess weight loss (EWL) of $53 \pm 27 \%$ and co-morbidity reduction of 54\%. 152 patients (39\%) out of 227 patients (58\%) with long-term complaints underwent revisional surgery. Main reasons for revision were weight regain and vomiting/food intolerance. Analysis before revision showed an outlet dilatation (17\%), pouch dilatation (16\%) and outlet stenosis (10\%). After revision, an additional EWL of $23 \%$ and $33 \%$ further reduction in co-morbidities was seen.

\section{Conclusion}

Primary VBG has an acceptable EWL of $53 \%$ and $55 \%$ of co-morbidities were improved. However, the high complication rate, often necessitating revision, underlines the limits of this procedure. 


\section{INTRODUCTION}

Over the last decades, morbid obesity is an increasing problem worldwide and will continue to increase even further in the next years. ${ }^{1}$ For obesity, there are many therapeutic options. The most efficacious therapy for morbid obesity is still bariatric surgery. One of the older techniques in bariatric surgery is the vertical banded gastroplasty (VBG), originally described by Mason in the early 1980 s. $^{2}$ Later, this technique was altered by MacLean et al. due to a high rate of staple line disruptions in the original technique. In this altered technique, the vertical stapler line is completely divided to reduce the chance of a gastrogastric fistula between the pouch and the gastric fundus. ${ }^{3}$

This gastroplasty establishes a restriction on food intake due to a small stomach pouch, which gives early satiety while maintaining passage of food through the further unaltered gastro-intestinal tract. This technique should avoid malabsorption of nutrients and medication. ${ }^{2,4}$ A VBG used to be a common restrictive procedure with good short-term results on excess weight loss and a low complication rate as shown in multiple studies. ${ }^{5-10}$ However, medium- and long-term results are more heterogeneous; from good long-term results ${ }^{6,9,11}$ to a high long-term complication rate up to $45 \%$ and a tendency for weight regain in the long-term follow-up, leading to revision rates between $21,4 \%$ and $65 \%{ }^{7,12-15}$ Due to the high rate of failure, this technique was abandoned in the Netherlands for several years in favor of other bariatric techniques. ${ }^{15}$

However, our interest in this old technique was renewed for two reasons. One is the recently published data by Bekheit et al. ${ }^{11}$ showing good long-term results, a low revision rate and an acceptable late complication rate after VBG, with the remark of the authors that good patient-selection is an important factor for long-term success. The second reason is the upcoming tendency of placing bands around bypasses and sleeves, in which a band is placed around the gastric pouch. This resembles a similar mechanical restriction as introduced in the VBG. ${ }^{16,17}$

The aim of the current study is to assess the long-term results of the MasonMacLean VBG's as a primary bariatric procedure. Assessment will include excess weight loss (EWL) percentage, early and late complications, revision rates and evolution of comorbidities. 


\section{MATERIALS AND METHODS}

\section{Methods}

Medical charts of all patients undergoing a primary VBG as described by Mason and MacLean ${ }^{2,3}$ between January 1998 and December 2008 at the Obesity Center, Catharina Hospital, the Netherlands, were retrieved. Data on patients' characteristics, operative details, evolution of excess weight loss and comorbidities, complications and outcome of revisions were collected and analyzed.

Excess weight was calculated as a percentage of the difference between the weight/BMl before surgery and the highest healthy weight $\left(B M l=25 \mathrm{~kg} / \mathrm{m}^{2}\right)$, according to the method as reported by Deitel et al. ${ }^{18} \mathrm{EWL}$ was expressed as a percentage of the amount of excess weight lost after surgery. Excess weight loss was categorized according to the Reinhold criteria. Using these criteria, an intervention is considered successful if patients lose at least $50 \%$ of their excess weight. $^{19}$

Since late 2011, the postoperative follow-up in the Catharina hospital is a structured 5-year program. However, subjects in this study only had a 1-year structured follow-up at the outpatient clinic and thereafter additional visits in case of complications. Further follow-up was to be performed by the general practitioner. To update current follow-up, a postal questionnaire was sent to all patients to retrieve the latest outcome. Patients who did not respond received a phone call.

The postal questionnaire contained questions about current and lowest weight after surgery, about comorbidities and use of medication, revisional surgery if applicable and possible complaints. Pre-defined complaints were dysphagia, frequent vitamin deficiencies and incisional hernia. Other groups of complications were composed out of reported complaints.

Evolution on comorbidities was divided into resolved (treatment no longer needed), improved (diminished amount of medication and/or use of CPAP in case of sleep apnea), stable, worsened or de novo. In case of complaints, patients were invited for additional analysis at the Obesity Center's outpatient clinic. In case of non-response, the data of the latest visit at the outpatient clinic were used as final outcome.

\section{Treatment}

All patients were assessed pre-operatively for eligibility for bariatric surgery by a multidisciplinary team consisting of a surgeon, a psychologist and a dietitian. When necessary, patients received additional psychological or dietary advice as 
preparation for the intervention and its consequences, aiming to increase the effect of the surgery. ${ }^{20}$

Surgery was performed by hand-assisted $(\mathrm{HA})$ laparoscopic approach in the earlier years and later by a pure laparoscopic approach. Using a CEEA21 stapler (Covidien, New Haven, CT, USA), a transgastric window is created about $6 \mathrm{~cm}$ distally from the gastro-esophageal junction along the lesser curvature. An oral gastric tube Ch34 is placed between the lesser curvature and the CEAA. Hereby, the pouch size is approximately $1.2 \mathrm{~cm}$. Along this tube, the vertical staple line is created from the transgastric window towards the angle of His using an EndoGIA. (Covidien, New Haven, CT, USA) For the final step of this procedure, a polytetrafluoroethylene (PTFE) band $(1.5 \times 7.5 \mathrm{~cm})$ is wrapped around the distal end of the gastric pouch, using the gastric tube for calibration. At a circumference of $6 \mathrm{~cm}$, the band is fixed using two non-absorbable sutures.

\section{Statistical analysis}

Data were retrospectively collected, managed and analyzed using SPSS version 22, for Windows (SPSS Inc, Chicago, IL, USA). Quantitative data are denoted as mean +/- standard deviation, whereas rates of complications, evolution on comorbidities are presented as a percentage. The Mann Whitney $U$ test was used to determine any significance of the observed differences among subgroups. Statistical significance was identified when the $p$ value was less than 0.05 . Summative figures and tables were used where necessary.

A sub-analysis was performed for laparoscopic versus HA-laparoscopic procedures and for non-super obese versus super obese patients when clinically relevant. Super obesity was considered with a BMI over $50 \mathrm{~kg} / \mathrm{m}^{2}$ according to the IFSO-guidelines.

No ethical approval was required for this study.

\section{RESULTS}

Between January 1998 and December 2008, a total of 392 consecutive patients underwent a VBG according to the technique described by Mason and MacLean. ${ }^{2,3}$ Since January 2009, no more VBG's were performed in our operating rooms. Two concomitant cholecystectomies were performed $(0.5 \%)$. In this population, $80.1 \%$ of patients was female. 155 laparoscopic and 235 HAlaparoscopic procedures were performed. One patient underwent a primary open procedure and one laparoscopic procedure was converted. Other baseline characteristics are displayed in Table 2.1. A total of 39 (9.9\%) super obese patients were identified in this study population. 
A total of 300 patients returned their completed questionnaires (76.5\%). Of those that did not return the questionnaire, ten patients $(2.6 \%)$ refused cooperation, 75 patients $(19.1 \%)$ could not be reached on multiple occasions due to various reasons and seven patients (1.8\%) died during the follow-up period, all due to causes unrelated to surgery. The average total follow-up of this population was $90 \pm 49$ months.

Intra-operative complications occurred in 17 patients (4.4\%). An intra-operative bleeding occurred in seven patients, a gastro-intestinal perforation was seen in four patients and a liver injury was reported in five patients, in one case leading to conversion to laparotomy. One patient had a splenic injury for which splenectomy after conversion to laparotomy was necessary.

Table 2.1 Baseline characteristics $(n=392)$

\begin{tabular}{lc}
\hline & Mean \pm SD \\
\hline Age (years) & $39.7 \pm 9.3$ \\
Male : Female $(\mathrm{n})$ & $78: 314$ \\
Body Mass Index $\left(\mathrm{kg} / \mathrm{m}^{2}\right)$ & $43.7 \pm 4.7$ \\
Diabetes Mellitus type $2(\mathrm{n}, \%)$ & $58(14.8 \%)$ \\
Diet only & $12(3.1 \%)$ \\
Oral medication & $31(7.9 \%)$ \\
Insulin dependent & $15(3.8 \%$ \\
Hypertension (n, \%) & $76(19.4 \%)$ \\
Dyslipidemia (n, \%) & $26(6.6 \%)$ \\
Sleep apnea (n, \%) & $7(1.8 \%)$ \\
Osteo-articular disease (n, \%) & $31(7.9 \%)$ \\
Procedure (n, \%) & $155(39.5 \%)$ \\
Laparoscopic & $235(59.9 \%)$ \\
Laparoscopic hand-assisted & $1(0.3 \%)$ \\
Open & $1(0.3 \%)$ \\
Laparoscopic converted to open & $95 \pm 27$ \\
Operative time (min) & $4 \pm 2$ \\
\hline
\end{tabular}

SD: standard deviation

Thirty patients (7.7\%) developed complications in the postoperative 30 -day period of whom eight (1.9\%) required a reoperation. Eight patients (1.9\%) had postoperative bleeding, of which three were successfully treated conservatively. Four patients (1.0\%) had postoperative leaking, treated by either surgical reintervention (three) or stenting (one). Three patients $(0.8 \%)$ developed a sepsis based on an intra-abdominal abscess, all were managed conservatively with antibiotics and two patients needed additional CT-guided drainage for a successful treatment. Other complications were pneumonia or urinary tract infection (1.0\%), pulmonary embolism (0.5\%), post-operative AV-nodal re-entry tachycardia $(0.3 \%)$, dehydration due to persistent vomiting with no proven evident cause $(0.3 \%)$ and deep venous thrombosis $(0.3 \%)$. One patient $(0.3 \%)$ 
developed necrosis of the stomach pouch, which necessitated debridement by reoperation and a prolonged admission at the intensive care unit. Her recovery was complicated by acute kidney failure and an ulcer in the recreated stomach pouch. Five patients $(1.3 \%)$ were treated for a trocar site / wound infection. There was no postoperative mortality.

At last follow-up, a total of 227 (58.4\%) patients have reported complaints after primary surgery. 152 patients (38.8\%) necessitated revision of the VBG for various reasons, of which 146 were operated in the Catharina hospital and 6 in other medical centers. 16 patients underwent a second revisional procedure. Amongst the 227 patients, the most common complaints after primary VBG were either vomiting, dysphagia and/or food intolerance $(47.3 \%)$, in eight patients caused by erosion of the band. Other common complaints were weight regain (28.8\%) and insufficient weight loss (15.9\%). Other complaints were severe reflux (2.7\%), which in one case caused a Barrett esophagitis, and (recurrent) abdominal pain (2.7\%). Six patients underwent revision in another hospital. Since no permission was given to retrieve their data, the complaints leading to revision of the primary VBG are unknown, but are taken into account in the total number of complaints. As mentioned before, seven patients deceased during follow-up; all due to causes unrelated to bariatric surgery.

\section{Weight loss}

The mean follow-up after VBG was $66.1 \pm 50.3$ months. The follow-up of patients necessitating revision was taken into account until revisional surgery was performed. The mean excess weight loss at last follow-up after a primary VBG was $51.2 \pm 27.4 \%$.

When analyzing weight loss results in patients with persistent VBG-anatomy, this study showed a mean EWL of $54.9 \pm 27.7 \%$. For patients who underwent revisional surgery, a significant lower mean \%EWL of $45.4 \pm 25.8 \%(p=0.001)$ was reported compared with the patients not necessitating revision.

According to Reinhold's 1982 criteria, only $48.2 \%$ of 392 patients achieved a successful long-term EWL-percentage (EWL $>50 \%)$.(19) Additional analysis of this study group shows that in patients without revisional surgery this percentage is $55.4 \%$ as opposed to only $36.8 \%$ of patients necessitating revision.

\section{Comorbidities}

Regarding the major obesity-related comorbidities, an improvement/ resolvement rate for type 2 diabetes of $70.7 \%$ was found. The rates for 
hypertension, dyslipidemia, sleep apnea and osteo-articular disease were respectively $40.8 \%, 46.1 \%, 71.4 \%$ and $54.8 \%$. This means, that after primary VBG, 53.5\% of the major obesity-related comorbidities were either improved or resolved, as shown in Table 2.2. The improvement/resolvement rate in patients with an intact VBG was $63.5 \%$ as opposed to $39.5 \%$ in the revision group.

Table 2.2 Results after primary VBG $(n=392)$.

\begin{tabular}{|c|c|c|c|c|}
\hline $\begin{array}{l}\text { Mean follow-up (months) } \\
\text { Mean BMI }\left(\mathrm{kg} / \mathrm{m}^{2}\right) \\
\text { Mean EWL }(\%)\end{array}$ & & & $\begin{array}{l}0.4 \\
6.0 \\
6.5 \\
\end{array}$ & \\
\hline & Worse & Stabile & Improved & Resolved \\
\hline Diabetes Mellitus type 2 (n) & 6 & 11 & 20 & 21 \\
\hline Hypertension (n) & 3 & 42 & 20 & 11 \\
\hline Dyslipidemia (n) & 3 & 11 & 5 & 7 \\
\hline Sleep apnea $(n)$ & 0 & 2 & 1 & 4 \\
\hline Osteo-articular disease (n) & 2 & 12 & 8 & 9 \\
\hline Total (\%) & 7.1 & 39.4 & \multicolumn{2}{|c|}{53.5} \\
\hline
\end{tabular}

SD: standard deviation; EWL: excess weight loss; BMI: body mass index.

\section{Revisions}

A total of 152 patients underwent revision, of which 16 patients eventually needed a second revision. Mean follow-up after revision was $56.5 \pm 37.8$ months. Average time between primary VBG and revision was $41.1 \pm 34.0$ months, ranging from 2-154 months. 21 patients underwent revision of the primary VBG, 16 patients were converted to a sleeve gastrectomy and in 115 patients, the primary VBG was converted to Roux-en-Y gastric bypass.

Total EWL after revisional surgery was $68.1 \pm 26.3 \%$ compared to the weight prior to primary bariatric surgery, which adds an additional excess weight loss of approximately $22.7 \%$ after revisional surgery. According to the Reinhold criteria, $77.0 \%$ of all patients had a good result after primary VBG combined with revisional surgery. ${ }^{19}$ Comorbidities showed a higher improvement/ resolvement rate at last follow-up after revision of $72.8 \%$

Additional analysis of the complaints before revision by means of either a stomach $\mathrm{x}$-ray, gastroscopy or both showed various technical reasons for failure of the VBG. 26 patients showed a wide outlet (17.1\%), pouch dilatation was seen in 24 patients (15.8\%) and 15 patients showed an outlet stenosis (9.9\%). Other technical reasons for failure were band erosion (5.3\%), band luxation $(2.0 \%)$, staple line dehiscence $(2.7 \%)$, pouch rotation $(0.7 \%)$ and band dehiscence $(0.7 \%)$. In the other patients (45.8\%), either no additional tests were performed or no evident technical cause for failure was found. 


\section{Subanalyses}

Subanalysis for laparoscopic procedures versus HA-laparoscopic procedures showed no significant difference in terms of intra- and postoperative complications, weight loss in terms of \%EWL, evolution of comorbidities and reasons for revision. Significantly more patients undergoing pure laparoscopy necessitated revision $(p=0.020)$.

No significant differences were found between non-super obese and super obese patients in terms of intra- and postoperative complications, evolution of comorbidities and necessitation for revision. A significantly better \%EWL was found in the group of non-super obese patients $(52.4 \%$ vs. $40.0 \%, p=0.001)$. Reasons for revision did not differ significantly except insufficient weight loss. It was more often found in super obese patients $(p=0.024)$.

\section{DISCUSSION}

Many studies have shown that a VBG is a good option for weight loss surgery in the short term, in terms of excess weight loss, reduction of co-morbidities and a low complication rate. ${ }^{2,5-10}$ The long-term results are heterogeneous. As mentioned before, good long-term results have been reported, however, various other studies have shown a high rate of long-term complications and a considerable revision rate. ${ }^{7,12,15,21}$ These results, in combination with the upcoming $A G B$ and later also the gastric sleeve eventually led to the abandonment of this restrictive procedure in the Netherlands. Nevertheless, the study by Bekheit et al. recently reported good long-term results with a good EWL ranging between $58 \%$ and $62 \%$ over the years, a low rate of long-term complications and a low revision rate $(5 \%) .{ }^{11}$ These good results were one of the reasons to re-establish interest and assess this center's experience with this bariatric procedure.

This study showed an acceptable mean long-term EWL of $52.5 \%$ after a primary VBG, accompanied by a reduction in co-morbidities of $53.5 \%$ with a mean follow-up of $66.1 \pm 50.4$ months. These results are comparable with various studies showing good results after VBG. ${ }^{4,6-11,22}$

The results of this study showed a comparable complication rate in the 30-day postoperative period of $7.7 \%$ as other studies analyzing results of laparoscopic VBGs have shown early complication rates of $4.2 \%-12.5 \%$. $^{8,9,12,23,24}$ These percentages are lower than those after open VBG as multiple studies show percentages over $15 \%$ after an open procedure, which can be explained by the higher incidence of wound infections. ${ }^{6,21,25}$ In this study population, a wound infection was found in only 5 patients (1.3\%), even though almost $60 \%$ of all 
patients underwent a hand-assisted laparoscopic procedure. In conclusion, in terms of weight loss, comorbidity improvement and/or resolvement and early complication rate, VBG is a good option as a restrictive bariatric procedure. Be that as it may, a long-term complication rate of $58 \%$ was found. Furthermore, a number of patients showed a possible technical reason for failure which might explain some of the long-term complaints, such as a wide outlet, outlet stenosis and pouch dilatation. A low incidence of staple line dehiscence of only $2.7 \%$ was found in this study. This can be explained by the surgical technique that was used, which is complete transection of the vertical stapler line, first described by MacLean et al. ${ }^{3}$ A total of 152 (38.8\%) necessitated revisional surgery after primary VBG. Analysis of the follow-up after revision showed an additional EWL of nearly $23 \%$ and an additional reduction in comorbidities of more than 33\%. Revision of the primary VBG seems feasible, but additional analysis of these procedures is needed for making a better conclusion on the outcome.

This series is not the first to report on the poor long-term results after VBG. Multiple studies have shown poor an outcome with high rates of revision, underlining the shortcomings of this type of procedure. ${ }^{7,12,15}$

In the present series, almost $8 \%$ of all patients' complaints necessitating revision were caused by problems with the band of the VBG (erosion, luxation or dehiscence). It is well-known that the VBG is not the only bariatric procedure in which a band is used to achieve long-term excess weight loss. The commonly used adjustable gastric band (AGB) and the more recently introduced banded gastric bypass and banded sleeve are other applications of a band in bariatric surgery. When regarding the upcoming banded bypass, certain anatomical similarities can be found between this new technique and the VBG. In both procedures, a band is placed at the end of a gastric pouch, with the aim of restricting food intake and thereby reaching a feeling of satiety faster. Buchwald et al. recently published a systematic review and meta-analysis on the outcome after banded gastric bypass. This review reported a weighted mean late complication rate of $20 \%$ after banded Roux-en- $Y$ gastric bypass. These recently published data, along with previous, mostly poor, long-term experiences after VBG and especially the similar anatomy between the VBG and the banded gastric bypass, should be taken into consideration when weighing the advantages and disadvantages of a banded gastric bypass.

Additional subanalyses showed no significant differences between laparoscopic and HA-laparoscopic procedures, except more revisions after laparoscopic procedures. Possible explanations might be better follow-up or psychologically more high-risk patients. Super obese patients showed a significant lower EWL and significantly more insufficient weight loss as reason for revision. Recent literature however shows significant variation in EWL and initial BMI: the higher 
the initial $\mathrm{BMI}$, the lower the eventual EWL, explaining the lower EWL in this study and the higher incidence of insufficient weight loss in revisional procedures. ${ }^{26}$

An underexposed topic in this study is hit when discussing the possible failure of the patients. In some patients, an evident technical cause was found for failure. Nevertheless, a large number of patients failed without a technical cause. A possible explanation could be the learning curve of the surgeons performing the VBGs, however the number of procedures performed make this unlikely. An unassessed topic in this study are the psychosocial factors and predictors which could influence weight loss and possibly the risk of failure after bariatric surgery. All patients underwent psychological screening preoperatively to exclude apparent psychological contra-indications. When necessary, patients received psychological treatment as preparation for the intervention and its consequences, aiming to increase the effect of the surgery. ${ }^{20}$ In the present study of 392 patients, a total of 101 patients returned to the outpatient clinic with complaints of either weight regain or insufficient weight loss. In some patients with these complaints, no evident technical cause was found or no additional analysis of these complaints was performed. Vallis et al. reported that if patients experience any psychological difficulties after surgical treatment with gastroplasty, these difficulties appear to be more related to the nature of morbid obesity than to the character of the individual. ${ }^{27}$ Furthermore, it is known that bariatric patients can develop an eating disorder after bariatric surgery which may eventually lead to a regain of weight. ${ }^{28}$ To acknowledge postoperative psychological difficulties, good follow-up is necessitated. This once again underlines the disadvantages of limited follow-up and might have left patients inadequately prepared to face the consequences of bariatric surgery. The limited follow-up of the patients in this study may be considered as a weak point towards the long-term outcome after VBG in this study. Better long-term results are mainly found in studies with a more intensive and extended follow-up period. ${ }^{6,9-11}$

Intensive follow-up will certainly improve long-term outcome, however a significant amount of patients in this study necessitating revision due to a technical failure of the primary VBG underlines the limitations of this old restrictive bariatric procedure. Nowadays, more effective and safe procedures have passed the VBG in the pecking order, due to a higher percentage EWL and a lower early complication rate. ${ }^{29,30}$

In conclusion: Although this study shows that VBG achieves an acceptable longterm EWL and a reduction in (the severity of) comorbidities, a high long-term complication rate, a high rate of failure and newly introduced bariatric surgery underline the limits of this old restrictive technique and strengthens the reason for abandoning this bariatric procedure in the Netherlands. 


\section{REFERENCES}

1. Selassie M, Sinha AC. The epidemiology and aetiology of obesity: a global challenge. Best Pract Res Clin Anaesthesiol. 2011;25(1):1-9.

2. Mason EE. Vertical banded gastroplasty for obesity. Arch Surg. 1982;117(5):701-6.

3. MacLean LD, Rhode BM, Forse RA. A gastroplasty that avoids stapling in continuity. Surgery. 1993;113(4):380-8.

4. Mason EE, Doherty C, Cullen JJ, Scott D, Rodriguez EM, Maher JW. Vertical gastroplasty: evolution of vertical banded gastroplasty. World J Surg. 1998;22(9):919-24.

5. Buchwald $H$, Avidor $Y$, Braunwald $E$, Jensen MD, Pories W, Fahrbach $K$, et al. Bariatric surgery: a systematic review and meta-analysis. JAMA. 2004;292(14):1724-37.

6. Lin YC, Chou FF, Chen SM, Wu CH. Vertical banded gastroplasty: a simple, effective and safe surgery for morbid obesity. Chang Gung Med J. 2003;26(10):754-60.

7. Miller K, Pump A, Hell E. Vertical banded gastroplasty versus adjustable gastric banding: prospective long-term follow-up study. Surg Obes Relat Dis. 2007;3(1):84-90.

8. Morino M, Toppino M, Bonnet G, Rosa R, Garrone C. Laparoscopic vertical banded gastroplasty for morbid obesity. Assessment of efficacy. Surg Endosc. 2002;16(11):1566-72.

9. Scozzari G, Toppino M, Famiglietti F, Bonnet G, Morino M. 10-year follow-up of laparoscopic vertical banded gastroplasty: good results in selected patients. Ann Surg. 2010;252(5):831-9.

10. Wang W, Yu PJ, Lee YC, Wei PL, Lee WJ. Laparoscopic vertical banded gastroplasty: 5-year results. Obes Surg. 2005;15(9):1299-303.

11. Bekheit M, Katri K, Salam WN, Ezzat T, El Kayal el S. Rejecting the demise of vertical-banded gastroplasty: a long-term single-institute experience. Obes Surg. 2013;23(10):1604-10.

12. Marsk R, Jonas E, Gartzios H, Stockeld D, Granstrom L, Freedman J. High revision rates after laparoscopic vertical banded gastroplasty. Surg Obes Relat Dis. 2009;5(1):94-8.

13. Schouten R, van Dielen FM, van Gemert WG, Greve JW. Conversion of vertical banded gastroplasty to Roux-en-Y gastric bypass results in restoration of the positive effect on weight loss and co-morbidities: evaluation of 101 patients. Obes Surg. 2007;17(5):622-30.

14. van Gemert WG, van Wersch MM, Greve JW, Soeters PB. Revisional surgery after failed vertical banded gastroplasty: restoration of vertical banded gastroplasty or conversion to gastric bypass. Obes Surg. 1998;8(1):21-8.

15. Schouten R, Wiryasaputra DC, van Dielen FM, van Gemert WG, Greve JW. Long-term results of bariatric restrictive procedures: a prospective study. Obes Surg. 2010;20(12):1617-26.

16. Awad W, Garay A, Martinez C. Ten years experience of banded gastric bypass: does it make a difference? Obes Surg. 2012;22(2):271-8.

17. Moon $R$, Teixeira $A$, Jawad MA. Pericardial patch ring Roux-en- $Y$ gastric bypass: a preliminary report. Obes Surg. 2013;23(4):480-5.

18. Deitel M, Greenstein RJ. Recommendations for reporting weight loss. Obes Surg. 2003;13(2): 159-60.

19. Reinhold RB. Critical analysis of long term weight loss following gastric bypass. Surg Gynecol Obstet. 1982;155(3):385-94.

20. Van Hout GC, Leibbrandt AJ, Jakimowicz JJ, Smulders JF, Schoon EJ, Van Spreeuwel JP, et al. Bariatric surgery and bariatric psychology: general overview and the Dutch approach. Obes Surg. 2003;13(6):926-31.

21. Suter $M$, Jayet $C$, Jayet $A$. Vertical banded gastroplasty: long-term results comparing three different techniques. Obes Surg. 2000;10(1):41-6; discussion 47.

22. Baltasar A, Bou R, Arlandis F, Martinez R, Serra C, Bengochea M, et al. Vertical banded gastroplasty at more than 5 years. Obes Surg. 1998;8(1):29-34.

23. Morino M, Toppino M, Bonnet G, del Genio G. Laparoscopic adjustable silicone gastric banding versus vertical banded gastroplasty in morbidly obese patients: a prospective randomized controlled clinical trial. Ann Surg. 2003;238(6):835-41; discussion 41-2. 
24. Nocca D, Aggarwal R, Blanc P, Gallix B, Di Mauro GL, Millat B, et al. Laparoscopic vertical banded gastroplasty. A multicenter prospective study of 200 procedures. Surg Endosc. 2007; 21(6):870-4.

25. van Dielen FM, Soeters PB, de Brauw LM, Greve JW. Laparoscopic adjustable gastric banding versus open vertical banded gastroplasty: a prospective randomized trial. Obes Surg. 2005; 15(9):1292-8.

26. van de Laar A. Bariatric Outcomes Longitudinal Database (BOLD) suggests excess weight loss and excess BMI loss to be inappropriate outcome measures, demonstrating better alternatives. Obes Surg. 2012;22(12):1843-7.

27. Vallis TM, Butler GS, Perey B, Veldhuyzen van Zanten SJ, MacDonald AS, Konok G. The role of psychological functioning in morbid obesity and its treatment with gastroplasty. Obes Surg. 2001;11(6):716-25.

28. van Hout G, van Heck G. Bariatric psychology, psychological aspects of weight loss surgery. Obes Facts. 2009;2(1):10-5.

29. Alexandrou A, Athanasiou A, Michalinos A, Felekouras E, Tsigris C, Diamantis T. Laparoscopic sleeve gastrectomy for morbid obesity: 5-year results. Am J Surg. 2014.

30. van Rutte PW, Smulders JF, de Zoete JP, Nienhuijs SW. Outcome of sleeve gastrectomy as a primary bariatric procedure. Br J Surg. 2014;101(6):661-8. 



\section{CHAPTER 3}

Medical and psychological predictors for longterm bariatric success using primary vertical banded gastroplasty as a model

M.R. van Wezenbeek

G.C.M. van Hout

S.W. Nienhuijs

Bariatr Surg Pract Patient Care. 2016;11:110-115 


\section{ABSTRACT}

\section{Introduction}

Bariatric surgery requires patients to implement permanent lifestyle changes which are affected by several factors. Psychological assessment seems to be essential for optimal weight loss and maintenance. The burden remains in identifying potential psychosocial predictors which might influence the longterm outcome. Therefore, this study was designed to identify those predictors for the long-term outcome after primary Vertical-Banded Gastroplasty (VBG).

\section{Methods \& Design}

Patients undergoing primary VBG between 2001 and 2004 completed a number of psychological questionnaires. Additional postal questionnaires were sent to retrieve the latest medical outcome. Patients were categorized as failed or successful based on their excess weight loss at last follow-up.

\section{Results}

This study identified a number of potential predictors. Failed patients showed more esthetic expectations, a more dominant character, more work absenteeism before surgery and depend more on the procedure than successful patients. Successful patients showed that positive emotions are an inhibitory factor for eating and less underwent revisional surgery.

\section{Conclusion}

This study shows a number of possible predictors, mainly found in the patient's character. The best way to prevent these predictors seems to be early recognition in the preoperative phase and a structured postoperative psychological follow-up to tackle any potential problems at an early stage. 


\section{INTRODUCTION}

Bariatric surgery has become an increasingly popular treatment option for morbid obesity. In 2011, over 340,000 procedures were performed, a number which is currently still growing. ${ }^{1}$ These days, bariatric surgery is not solely performed to achieve long-term weight loss anymore, as more patients are operated for metabolic diseases like diabetes mellitus type 2. ${ }^{2,3}$ Bariatric surgery requires patients to implement permanent lifestyle changes to achieve longterm successful outcomes. ${ }^{4}$ These lifestyle changes are affected by several nonsurgical factors such as eating behavior, personality, socio-economic status and coping.-7 $^{5-7}$

Psychological assessment both pre- and postoperative appears to be essential to guarantee an optimal weight loss and weight loss maintenance after bariatric surgery. ${ }^{8}$ However, there are still countries in which the role of the mental health providers is not well defined and the psychological support is not standard in the current postoperative standard package of care. ${ }^{9}$

Results are contradictory and far from conclusive and there is no general consensus on which psychological factors may predict the outcome after bariatric surgery. The challenge remains in identifying these potential psychological factors which might influence the long-term outcome. ${ }^{10}$ An important impediment is the overall methodological weakness, the different outcomes measures for success, small patient groups and diverse lengths of follow-up of the current literature to find reliable and valid predictors for the outcome of various bariatric procedures. ${ }^{6}$ There are various bariatric procedures. Malabsorptive procedures like Roux-en-Y gastric bypass (RYGB) and duodenal switch and restrictive procedures like adjustable gastric banding $(A G B)$, sleeve gastrectomy (SG) and vertical-banded gastroplasty (VBG). The VBG is an old restrictive procedure, originally invented by Mason et al, later altered by MacLean. ${ }^{11,12}$ Studies on this procedure show good short-term results, however long-term results were more heterogeneous, which eventually led to the abandonment of this procedure in the Netherlands some years ago. ${ }^{13-15}$

Research on early postoperative predictors were published by a part of the current group. 6,16,17 The current study was designed to identify potential psychosocial predictors for the long-term outcome after primary VBG. 


\section{METHODS AND DESIGN}

\section{Methods}

Between January 2001 and November 2004, bariatric patients undergoing VBG at the Obesity Center, Catharina hospital, Eindhoven, the Netherlands, were included and asked to complete several questionnaires pre-operatively and six months, one year and two years postoperatively. All patients undergoing primary VBG were included. The questionnaires included questions to assess personality, eating behavior, obesity-related beliefs, health-related quality of life (HRQoL), body attitude, psychological and somatic symptoms and coping. All questionnaires are displayed in Table 3.1. Furthermore, patients were asked to complete questions on motivation, expectations, the history of their obesity, eating behavior and several eliciting and inhibitory factors with regard to eating.

Table 3.1 Psychological Questionnaires

\begin{tabular}{|c|c|}
\hline Symptom Checklist & SCL-90 ${ }^{18}$ \\
\hline Dutch Personality Questionnaire & $\mathrm{DPQ}^{19}$ \\
\hline Dutch Eating Behavior Questionnaire & $\mathrm{DEBQ}^{20}$ \\
\hline Body Attitude Test & BAT $^{21}$ \\
\hline RAND-36 Health Survey & RAND $-36^{22}$ \\
\hline Amsterdam Biographical Questionnaire & $\mathrm{ABQ}^{* 23}$ \\
\hline Eating Disorder Inventory II & EDI-II* 24 \\
\hline Utrecht Coping List & $\mathrm{UCL}^{* 25}$ \\
\hline Multidimensional Health Locus of Control scale & $\mathrm{MHLC}^{\star 26}$ \\
\hline Multidimensional Health Locus of Control-obesity & MHLC-obesity ${ }^{\star} 27$ \\
\hline Overweight Cognition Questionnaire & $\mathrm{OCG}^{*} 28$ \\
\hline
\end{tabular}

*Questionnaires only administered pre-operatively.

Since the end of the year 2011, follow-up is a structured five-year program at this center. However, patients participating in the current study underwent limited follow-up during one year in the hospital, after which they were referred back to the general practitioner in case of no complaints after one year. To retrieve all medical outcome and the latest follow-up, all medical charts were reviewed and all patients that underwent primary VBG in this center received a postal questionnaire. Non-responding patients received a phone call. In case of non-response, the last known follow-up out of the medical charts was used.

The postal questionnaire was designed to retrieve the current and the lowest weight after surgery, current status of obesity-related comorbidities and use of medication, revisional surgery when applicable and long-term complaints. 
Complaints were categorized into dysphagia, frequent vitamin deficiencies, incisional hernia and other complaints with an open text field.

The comorbidities were categorized into resolved (treatment no longer needed), improved (diminished amount of medication and/or use of CPAP in case of sleep apnea), stable, worsened or de novo. Patients reporting complaints were invited for additional analysis at the outpatient clinic.

Based on their last follow-up data, patients were categorized, depending on the percentage of excess weight loss (\%EWL), as failed patient ( $<50 \%)$ or successful patient $(\geq 50 \%)$. These percentages are used in the Reinhold criteria, which state that a bariatric procedure is considered successful when a \%EWL is achieved of at least $50 \%{ }^{18}$ Patients necessitating revision were considered failed patients, unless there was an evident technical cause for failure. In case of technical failure, patients were considered failed or successful based on the \%EWL at last follow-up after VBG.

For this study, analysis of all pre-operative questionnaires was performed to identify possible predictors for long-term success after primary VBG. Long-term results after VBG were completed with the use of the additional postal questionnaires.

\section{Treatment}

All patients were evaluated at the outpatient clinic for eligibility for bariatric surgery by a dietitian, a bariatric nurse and a psychologist. When eligible for surgery, patients were asked to fill in all pre-operative questionnaires. Surgery was performed by hand-assisted (HA) laparoscopic approach. A transgastric window was created, using a CEEA21 stapler (Covidien, New Haven, CT, USA), about $6 \mathrm{~cm}$ distally from the gastro-esophageal junction along the lesser curvature. An oral gastric tube Ch34 was placed between the lesser curvature and the CEAA. Hereby, the pouch size is approximately $1.2 \mathrm{~cm}$ in length, measured from the gastro-esophageal junction. Along this tube, a vertical staple line was created from the transgastric window towards the angle of His using an EndoGIA. (Covidien, New Haven, CT, USA) For the final step of this procedure, a polytetrafluoroethylene (PTFE) band $(1.5 \times 7.5 \mathrm{~cm})$ was wrapped around the distal end of the gastric pouch, using the gastric tube for calibration. At a circumference of $6 \mathrm{~cm}$, the band is fixed using two nonabsorbable sutures.

\section{Statistical analysis}

Collection, management and analysis of all data was done retrospectively using SPSS version 22, for Windows (SPSS Inc, Chicago, IL, USA). Quantitative data are 
denoted as mean +/- standard deviation, whereas nominal data is denoted as a percentage. The Mann Whitney U-test was used to determine any significance of the observed differences among groups. Statistical significance was identified when the $p$ value was less than 0.05. Summative figures and tables were used where necessary.

No ethical approval was required for this study and this study was performed according to the principles of the Declaration of Helsinki. Informed consent was obtained from subjects.

\section{RESULTS}

A total of 98 patients were eligible for inclusion for this study. 70 patients (71.4\%) completed and returned their postal questionnaires. The other 28 patients did not complete the questionnaire after postal non-response. 18 phone numbers were out of order, one patient refused cooperation and nine patients could not be reached on multiple occasions. The total group showed an average total follow-up of $106.9 \pm 49.3$ months with an average total \%EWL of $52.9 \pm 27.8 \%$ at last follow-up after VBG. When classifying patients as described in the Methods, 62 failed patients (FP) and 36 successful patients (SP) were identified. Table 3.2 displays the baseline characteristics.

Table 3.2 Baseline characteristics $(n=98)$.

\begin{tabular}{|c|c|c|c|}
\hline & $\begin{array}{c}\text { Failed patients } \\
n=62(63.3 \%) \\
\text { Mean } \pm \text { SD }\end{array}$ & $\begin{array}{c}\text { Successful patients } \\
n=36(36.7 \%) \\
\text { Mean } \pm \text { SD }\end{array}$ & $p$-value \\
\hline Age (years) & $39.4 \pm 8.5$ & $39.3 \pm 7.7$ & 0.836 \\
\hline Male : Female $(n)$ & $9: 53$ & $2: 34$ & 0.319 \\
\hline Body Mass Index before VBG $\left(\mathrm{kg} / \mathrm{m}^{2}\right)$ & $46.4 \pm 5.3$ & $44.3 \pm 4.4$ & 0.039 \\
\hline \multicolumn{4}{|l|}{ Pre-operative comorbidities } \\
\hline Diabetes Mellitus type 2 (n) & 7 & 4 & 1.000 \\
\hline Hypertension (n) & 15 & 9 & 0.929 \\
\hline Dyslipidemia (n) & 3 & 1 & 1.000 \\
\hline Sleep apnea $(n)$ & 1 & 0 & 1.000 \\
\hline Osteo-articular disease (n) & 6 & 1 & 0.256 \\
\hline Operative time (min) & $94.4 \pm 24.2$ & $90.3 \pm 18.5$ & 0.599 \\
\hline Length of hospital stay (days) & $3.1 \pm 1.2$ & $3.1 \pm 1.0$ & 0.906 \\
\hline Number of patients with $\geq 1$ comorbidity $(n)$ & 24 & 13 & 0.798 \\
\hline
\end{tabular}

VBG: vertical banded gastroplasty; SD: standard deviation. 


\section{Medical results}

Shown in Table 3.3 are the medical results at last known follow-up after VBG and after revision, including follow-up, \%EWL, number of revisional procedures and the presence of any comorbidities. Furthermore, the table shows the \%EWL as expected preoperatively by the patients. Patients who underwent revision, had either a revision of the primary VBG $(n=3)$, conversion to sleeve gastrectomy $(n=2)$ or conversion to Roux-en-Y gastric bypass $(n=18)$. Main reason for revision in the entire study population was weight regain (47.8\%), which in one case was caused by an evident technical cause (band dehiscence). Mean \%EWL of the total population at last known follow-up including any necessitated revision was $60.7 \pm 20.8 \%$.

Table 3.3 Medical results at last follow-up $(n=98)$.

\begin{tabular}{lccc}
\hline & $\begin{array}{c}\text { Failed patients } \\
\mathbf{n = 6 2}(\mathbf{6 3 . 3 \% )} \\
\text { Mean } \pm \text { SD }\end{array}$ & $\begin{array}{c}\text { Successful patients } \\
\mathbf{n = 3 6}(\mathbf{3 6 . 7 \% )} \\
\text { Mean } \pm \text { SD }\end{array}$ & $\boldsymbol{p}$-value \\
\hline Mean follow-up without revision (months) & $95.7 \pm 48.3$ & $87.4 \pm 58.0$ & 0.527 \\
Mean EWL without revision (\%) & $30.4 \pm 18.0$ & $74.1 \pm 25.1$ & $<\mathbf{0 . 0 0 1}$ \\
Number of revisions (n) & 20 & 3 & $\mathbf{0 . 0 0 7}$ \\
Mean total follow-up (months) & $115.1 \pm 42.4$ & $92.9 \pm 57.2$ & 0.143 \\
Mean EWL at last follow-up (\%) & $39.0 \pm 23.0$ & $77.5 \pm 15.8$ & $<\mathbf{0 . 0 0 1}$ \\
Number of patients with $\geq 1$ comorbidity & 10 & 3 & 0.362 \\
Expected EWL before surgery (\%) & $79.3 \pm 22.7$ & $84.3 \pm 26.2$ & 0.369 \\
\hline
\end{tabular}

SD: standard deviation; EWL: excess weight loss

\section{Questionnaires}

Results of all questionnaires mentioned in the Methods were reviewed for potential predictors.

Table 3.4 shows the clinically relevant and the significantly different parameters of the various questionnaires. No significant differences were seen between the two groups in the SCL-90 subcategories, the UCL, the EDI (assessing the level of emotional eating), the MHLC, the NVE and the OCL-25.

Table 3.4 Results Psychological Questionnaires $(n=98)$.

\begin{tabular}{llccc}
\hline & $\begin{array}{c}\text { Failed patients } \\
\mathbf{n = 6 2}(\mathbf{6 3 . 3 \% )} \\
\mathbf{M e a n} \pm \mathbf{S D}\end{array}$ & $\begin{array}{c}\text { Successful patients } \\
\mathbf{n = 3 6}(\mathbf{3 6 . 7 \% )} \\
\mathbf{M e a n} \pm \mathbf{~ S D}\end{array}$ & $\boldsymbol{p}$-value \\
\hline SCL-90 & Psychoneuroticism & $139.0 \pm 39.4$ & $145.5 \pm 45.2$ & 0.611 \\
DPQ & Dominance & $14.8 \pm 6.5$ & $12.1 \pm 5.8$ & $\mathbf{0 . 0 4 9}$ \\
ABV & Work absenteeism (days) & $24.3 \pm 58.4$ & $18.8 \pm 65.9$ & $\mathbf{0 . 0 2 5}$ \\
LAV & Total score & $65.5 \pm 15.2$ & $63.9 \pm 17.1$ & 0.622 \\
RAND-36 & Role limitations (emotional) & $5.5 \pm 1.0$ & $5.0 \pm 1.2$ & $\mathbf{0 . 0 3 2}$ \\
RAND-36 & General perception of health & $15.0 \pm 3.9$ & $14.9 \pm 3.8$ & 0.804 \\
MHLC-Obesity & Doctor as locus of control & $11.4 \pm 2.5$ & $12.7 \pm 3.0$ & $\mathbf{0 . 0 3 8}$ \\
\hline
\end{tabular}

SD: standard deviation 


\section{Motivation}

Patients were presented a number of options on what motivated them to lose weight and what motivated them to choose for a bariatric procedure. These questionnaires were answered in a yes/no-format. Options on motivations to lose weight were health, limitations in movement, social reasons, esthetic reasons, condition, dissatisfaction with body and other psychic reasons. Motivations on choosing a bariatric procedure were insufficient results with other weight-losing methods, lasting weight reduction, the ability to eat less, surgery acting as a driving force and faster satiety.

No significant differences were found in any of the options between the two groups. Regrouping the motivations did not change the outcome between the two groups.

\section{Expectations}

The questions on expectations were similar to those for motivation. Options on expectations of the effects of the bariatric procedure were faster satiety, change of eating pattern, a happier life, lasting weight reduction, procedure acting as a driving force, increased activity, the ability to buy new clothes and other expectations. The study showed that patients in the FP group significantly more often expected to be able to buy new clothing $(p=0.004)$. No significant differences were seen with respect to the other expectations.

\section{History of patients' obesity}

All patients were asked about their weight status (underweight, normal weight, overweight) during various life phases. No significant differences were found between the two groups. Furthermore, no significant differences were seen in the causes for the obesity, such as pregnancy, a medical reason, eating pattern or genetic predisposition.

\section{Eliciting and inhibitory factors}

There can be many eliciting as well as inhibitory factors for eating more than normal. Several eliciting factors were reviewed, however, no factors were significantly different between the two groups. A few only showed a trend to occur more often in the FP group, such as social activities $(p=0.074)$ and the confrontation with food $(p=0.088)$. Other factors reviewed were emotions, boredom, social control, watching television, time to eat and appetite. Inhibitory factors were positive and negative emotions, dieting, social control and work. A significant difference was seen in the positive emotions $(p=0.024)$ 
in favor of the SP group. No significant differences were seen for the other inhibitory factors.

\section{Eating behavior}

No significant difference was seen in the occurrence of binge eating or grazing between the two groups. Furthermore, no differences were seen between two groups when assessing number of meals, number of snacks and the amount of calories. Eating has many functions. This study assessed eating to attenuate (negative) emotions, to maintain positive emotions, satiety, distraction, habit, sociability, to stay alive and as a reaction to problems. No significant differences were seen between groups on incidence of these various functions.

\section{DISCUSSION}

This study was designed to find potential predictors for long-term success after primary vertical-banded gastroplasty, an old restrictive procedure achieving an average $\% E W L$ of up to $60 \% .{ }^{19}$ Of all patients, $63.2 \%$ were considered a failed patient, underlining the inferiority of this procedure as shown in other studies before. $^{13-15}$ Partly, failure may be imputed to the limited follow-up these patients had postoperatively, even though literature suggests a high long-term complication rate after VBG and a high rate of revision. As stated before, identifying predictors on the outcome after bariatric surgery remains a burden. Therefore, this study was set up to find either medical or psychosocial predictors on the long-term success after a primary bariatric procedure.

Considering the medical results, it is noteworthy that the pre-operative BMI is significantly higher in the FP group. This might suggest that a higher BMI compared to a lower $\mathrm{BMI}$ within the group of eligible bariatric patients may increase the chance of failure in terms of long-term \%EWL, supporting multiple previously reported results. ${ }^{20,21}$ Even though the initial BMI influences the amount of excess weight and thus the percentage of \%EWL, this study shows far better results in the SP group. ${ }^{22} \% E W L$ at last follow-up was nearly twice as much in the SP group as opposed to the FP group. This study shows more revisional procedures in the FP group, which is explained by the occurrence of failure and the way of categorizing patients. The best option for failed procedures is a revisional procedure, so it seems only logical that the revision rate is higher in the FP group.

Other medical and demographic parameters such as comorbidities, gender, excess weight, length of hospital stay and operating time do not seem to be a predictor for the long-term results in this study population. 
Considering the patients' pre-operative motivation for losing weight and choosing for a bariatric procedure, no significant differences were found. This suggests that various reasons for losing weight or for choosing a bariatric procedure are no predictor for the outcome after primary VBG in this study. Previous studies however, have shown that a poor health as motivation can be a potential predictor for long-term success. ${ }^{23}$ It remains to what extend the reason for losing weight is related to the cause of the developed obesity. This study showed no differences between the groups in terms of reasons for obesity.

In terms of pre-operative expectations, the only potential predictor may be the fact that patients in the FP group significantly more often expected to buy new clothing in the postoperative phase, a pure esthetic expectation of the procedure. This supports old data published on esthetic expectations after bariatric surgery, stating that patients with a lower \%EWL more often have high esthetic expectations as opposed to successful patients. ${ }^{24}$ No differences were seen in expected \%EWL before surgery between the two groups.

The studied population showed a similar weight history. The phase in which the overweight developed did not differ significantly between the FP group and the SP group. Furthermore, no differences were seen in terms of various options or number of weight loss attempts, which strengthens previous results on weight loss attempts before bariatric surgery. ${ }^{20}$

There are many known eliciting factors for (excessive) eating, with emotions being a common factor. Multiple studies have shown that emotional eating has either no effect or a negative effect on postoperative weight loss. ${ }^{20}$ This study showed that emotions as a eliciting factor for eating had no influence on the long-term success after primary VBG.

A significant difference was found for the predictive value of positive emotions as an inhibitory factor for eating in favor of the patients in the SP group. It may be expected that the vast majority of patients have more positive emotions after bariatric surgery, since bariatric procedures haven shown to improve quality of life, body dissatisfaction, depression and anxiety symptoms and eating disorders. ${ }^{16,25-27}$ It furthermore can be expected that patients will be confronted with more positive emotions post-bariatric surgery and thus, in patients reporting positive emotions as an inhibitory factor, might lead to a higher percentage of \%EWL.

This study showed no predictive value in any of the parameters regarding eating behavior. Eating behavior, in terms of amount of calories, number of meals per day, binge eating, grazing and function of eating, will have a no predictive value on the percentage of long-term \%EWL after primary VBG based on the results from this study. The literature is diverse on the effect of mainly eating and grazing. Some studies report a negative relation between 
binge eating and \%EWL, others do not report binge eating as a negative predictive value. ${ }^{28,29}$ This study supports the latter conclusion of the two.

Several questionnaires were used in this study. Only a few potential predictors can be pointed out with the current results. These results suggest that a more dominant character according to the DPQ leads to an inferior percentage of long-term \%EWL. A dominant character is confident, authoritarian and likes to make its own decisions. Earlier studies showed no predictive value for shortterm \%EWL, using the same questionnaire. ${ }^{21}$

Patients in the FP group showed a higher rate of work absenteeism one year prior to bariatric surgery. A possible explanation for this outcome may be a poorer physical or mental state of the patient before surgery, however this hypothesis is not supported by the other results on physical and mental health in this study and extensive research showed no previous data on this specific subject.

Multiple studies have shown that an emotional eating pattern is a possible negative predictor for weight loss, while others support the current results, in which no predictive value on weight loss was found for emotional eating. ${ }^{20}$

A striking finding is the fact that successful patients in this study believe that the VBG is significantly more responsible for long-term success as opposed to the FP group, according to the MHCL-obesity questionnaire. A result suggesting that successful patients place the long-term outcome more in the hands of the doctor and thus appear to be more receptive to lifestyles changes necessitated for long-term successful weight loss. It is strongly suggested that postoperative weight loss is largely dependent on making and sustaining changes in eating and physical activity. ${ }^{30}$ This finding strengthens the believe that psychological follow-up is important for the long-term success after bariatric surgery. Furthermore, it appears of the utmost importance that patients are well informed pre-operatively that lifestyle changes are a key factor in achieving a successful long-term excess weight loss.

Results from this study should be interpreted with caution when translating them to current practice. A VBG is an old procedure, only a few are performed nowadays since this procedure is surpassed by newer options like the adjustable gastric band and the sleeve gastrectomy. ${ }^{1}$ Even though, it is the authors' believe that this group of bariatric patients is not different to any other patient group undergoing a more modern restrictive procedure. Disadvantages of this study are the limited number of patients, the retrospective design of the study and the low frequency follow-up after surgery, which may have influenced the results. More intensive follow-up might have improved the longterm success rate and thereby have changed the categorization of patients in this study. An advantage of this study is the fact that it is not a controlled trial and it might thereby be a better reflection on everyday practice. 
Nowadays, psychological assessment has found a permanent place in both the preoperative and the postoperative phase of a bariatric procedure. Be that as it may, it remains a burden to find potential hazards for poor long-term outcome after bariatric surgery.

When analyzing the possible predictors for long-term success it seems that predictors for successful \%EWL can mainly be found in the patient's character. This study showed more than five possible psychosocial predictors and it seems impossible to tackle all of them preoperatively. Obviously, preoperative psychological screening remains inevitable for identifying known contraindications for bariatric surgery and possible predictors for the long-term outcome, but structured psychological follow-up seems more important to improve the rate of long-term success after bariatric surgery. Evidence on predictors remains limited and more elaborate research and prospective studies are necessitated to gain more evidence on this subject.

\section{Conclusions}

This study shows a number of possible predictors, mainly found in the patient's character and the best way to prevent these predictors seems to be early recognition in the preoperative phase and a structured postoperative psychological follow-up to tackle any potential problems at an early stage. 


\section{REFERENCES}

1. Buchwald H, Oien DM. Metabolic/bariatric surgery worldwide 2011. Obes Surg. 2013;23(4):427-36.

2. Ribaric G, Buchwald JN, McGlennon TW. Diabetes and weight in comparative studies of bariatric surgery vs conventional medical therapy: a systematic review and meta-analysis. Obes Surg. 2014;24(3):437-55.

3. Sjostrom L. Bariatric surgery and reduction in morbidity and mortality: experiences from the SOS study. Int J Obes (Lond). 2008;32 Suppl 7:S93-7.

4. Puzziferri N. Psychologic issues in bariatric surgery--the surgeon's perspective. Surg Clin North Am. 2005;85(4):741-55.

5. Hsu LK, Benotti PN, Dwyer J, Roberts SB, Saltzman E, Shikora S, et al. Nonsurgical factors that influence the outcome of bariatric surgery: a review. Psychosom Med. 1998;60(3):338-46

6. van Hout GC, Hagendoren CA, Verschure SK, van Heck GL. Psychosocial predictors of success after vertical banded gastroplasty. Obes Surg. 2009;19(6):701-7.

7. Mathus-Vliegen EM. Long-term health and psychosocial outcomes from surgically induced weight loss: results obtained in patients not attending protocolled follow-up visits. Int J Obes. 2007;31(2):299-307.

8. Pataky Z, Carrard I, Golay A. Psychological factors and weight loss in bariatric surgery. Curr Opin Gastroenterol. 2011;27(2):167-73.

9. Ogden J, Hollywood A, Pring C. The Impact of Psychological Support on Weight Loss Post Weight Loss Surgery: a Randomised Control Trial. Obes Surg. 2015 Mar;25(3):500-5.

10. Waumsley A, Atter N, Boyle S, et a. Obesity Working Group 2011: Obesity in the UK: A psychological perspective. In: Society TBP, editor. 2011.

11. Mason EE. Vertical banded gastroplasty for obesity. Arch Surg. 1982;117(5):701-6.

12. MacLean LD, Rhode BM, Forse RA. A gastroplasty that avoids stapling in continuity. Surgery. 1993;113(4):380-8

13. Marsk R, Jonas E, Gartzios H, Stockeld D, Granstrom L, Freedman J. High revision rates after laparoscopic vertical banded gastroplasty. Surg Obes Relat Dis. 2009;5(1):94-98.

14. Miller K, Pump A, Hell E. Vertical banded gastroplasty versus adjustable gastric banding: prospective long-term follow-up study. Surg Obes Relat Dis. 2007;3(1):84-90.

15. Schouten R, Wiryasaputra DC, van Dielen FM, van Gemert WG, Greve JW. Long-term results of bariatric restrictive procedures: a prospective study. Obes Surg. 2010;20(12):1617-26.

16. van Hout GC, Fortuin FA, Pelle AJ, Blokland-Koomen ME, van Heck GL. Health-related quality of life following vertical banded gastroplasty. Surg Endosc. 2009;23(3):550-6.

17. van Hout $G$, van Heck G. Bariatric psychology, psychological aspects of weight loss surgery. Obes Facts. 2009;2(1):10-5.

18. Reinhold RB. Critical analysis of long term weight loss following gastric bypass. Surg Gynecol Obstet. 1982;155(3):385-94.

19. Bekheit M, Katri K, Salam WN, Ezzat T, El Kayal el S. Rejecting the demise of vertical-banded gastroplasty: a long-term single-institute experience. Obes Surg. 2013;23(10):1604-10.

20. Livhits M, Mercado C, Yermilov I, Parikh JA, Dutson E, Mehran A, et al. Preoperative predictors of weight loss following bariatric surgery: systematic review. Obes Surg. 2012;22(1):70-89.

21. Larsen JK, Geenen R, Maas C, de Wit P, van Antwerpen T, Brand N, et al. Personality as a predictor of weight loss maintenance after surgery for morbid obesity. Obes Res. 2004;12(11):1828-34.

22. van de Laar A. Bariatric Outcomes Longitudinal Database (BOLD) suggests excess weight loss and excess BMI loss to be inappropriate outcome measures, demonstrating better alternatives. Obes Surg. 2012;22(12):1843-7.

23. Vallis MT, Ross MA. The Role of Psychological Factors in Bariatric Surgery for Morbid Obesity: Identification of Psychological Predictors of Success. Obes Surg. 1993;3(4):346-59 
24. Bleijenberg G, Raes BC, Heevel JG. [Psychological aspects of gastric partitioning, surgical treatment of patients with extreme obesity; preliminary results]. Ned Tijdschr Geneeskd. 1986;130(15):693-696

25. Kolotkin RL, Crosby RD, Gress RE, Hunt SC, Adams TD. Two-year changes in health-related quality of life in gastric bypass patients compared with severely obese controls. Surg Obes Relat Dis. 2009;5(2):250-6.

26. Sanchez Zaldivar S, Arias Horcajadas F, Gorgojo Martinez JJ, Sanchez Romero S. [Evolution of psychopathological alterations in patients with morbid obesity after bariatric surgery]. Med Clin. 2009;133(6):206-12.

27. Thonney B, Pataky Z, Badel S, Bobbioni-Harsch E, Golay A. The relationship between weight loss and psychosocial functioning among bariatric surgery patients. Am J Surg. 2010;199(2):183-8.

28. Powers PS, Perez A, Boyd F, Rosemurgy A. Eating pathology before and after bariatric surgery: a prospective study. Int J Eat Disord. 1999;25(3):293-300

29. Sheets CS, Peat CM, Berg KC, White EK, Bocchieri-Ricciardi L, Chen EY, et al. Post-operative Psychosocial Predictors of Outcome in Bariatric Surgery. Obes Surg. 2015;25(2):330-45

30. Kalarchian $M$, Turk $M$, Elliott J, Gourash W. Lifestyle management for enhancing outcomes after bariatric surgery. Curr Diab Rep. 2014;14(10):540

31. Arrindell W, Ettema J. SCL-90. Handleiding bij een multidimensionele psychopathologieindicator. [SCL-90. Manual for a multidimensional psychopathology-indicator]. Lisse: Swets \& Zeitlinger, 2003.

32. Luteijn F, Starren J, van Dijk H. Handleiding NPV [Manual DPQ]. Lisse: Swets \& Zeitlinger, 2000.

33. van Strien T, Frijters J, Bergers G, al. e. Handleiding Nederlandse Vragenlijst voor Eetgedrag [Manual Dutch Eating Behavior Questionnaire]. Lisse: Swets \& Zeitlinger, 1986.

34. Probst M, Vandereycken W, van Coppenolle H, al. e. Body Attitude Test for patients with an eating disorder: psychometric characteristics of a new questionnaire. Eat Disord 1995;3: 133-145

35. van der Zee K, Sanderman R. Het meten van de algemene gezondheidstoestand met de RAND-36. Een handleiding [Measuring of general health-state with the RAND-36. A manual]. Groningen: Noordelijk Centrum voor Gezondheidsvraagstukken/RUG, 1993.

36. Wilde G. Neurotische labiliteit gemeten volgens de vragenlijstmethode. [Neurotic lability measured by the questionnaire method]. Amsterdam: Van Rossen, 1970.

37. Van Strien T. Handleiding EDI-II-NL [Manual EDI-II-NL]. Lisse: Swets \& Zeitlinger, 2000.

38. Schreurs $P$, van de Willige G, Tellegen B, al. e. De Utrechtse Coping Lijst. Omgaan met problemen en gebeurtenissen. Handleiding [The Utrecht Coping List. Coping with problems and events. Manual]. Lisse: Swets \& Zeitlinger, 1988.

39. Halfens R, Philipsen H. Een gezondheidsspecifieke beheersingsoriëntatieschaal: Validiteit en betrouwbaarheid van de MHLC. [Health specific locus of control scale: Validity and reliabilty of the MHLC]. Tijdschrift Soc Gezondheidsz 1988;66:399-403

40. Larsen J, Geenen R. Een beheersingsoriëntatieschaal voor obesitas [Locus of control scale for obesity]. Internal publication, Department of Health Psychology, Utrecht University, The Netherlands. 2000

41. Zijlstra H, Larsen JK, van Ramshorst B, Geenen R. The association between weight loss and self-regulation cognitions before and after laparoscopic adjustable gastric banding for obesity: a longitudinal study. Surgery. 2006;139(3):334-9. 


\section{CHAPTER 4}

Long-term results after revisions of failed primary vertical banded gastroplasty

M.R. van Wezenbeek

J.F. Smulders

J.P.J.G.M. de Zoete

M.D. Luyer

G. van Montfort

S.W. Nienhuijs

World J Gastrointest Surg. 2016;8(3):238-45

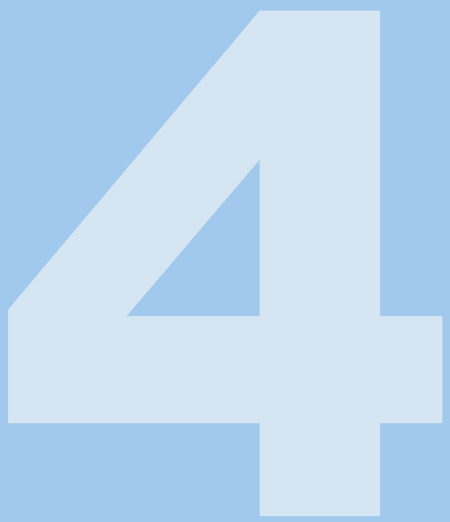




\section{ABSTRACT}

\section{Background \& Objectives}

Vertical banded gastroplasty (VBG) was a popular restrictive bariatric procedure however, has been abandoned due to a high long-term complications rate in many cases leading to the necessitation of revisional surgery. As a number of these revisions can be expected, this study reports and compares the results after revision of the primary VBG (Re-VBG), conversion to sleeve gastrectomy (cSG) and conversion to Roux-en-Y gastric bypass (cRYGB).

\section{Setting \& Methods}

In this retrospective single-center study, all patients with a failed VBG who underwent revisional surgery were included. Medical charts were reviewed and additional postal questionnaires were sent to update follow-up.

Results

A total 152 patients were included in this study, of which 21 underwent ReVBG, 16 underwent CSG and 115 patients underwent CRYGB. 16 patients necessitated a second revisional procedure. No patients were lost-to-follow-up. Two patients deceased during the follow-up period, 23 patients did not return the questionnaire. Main reasons for revision were dysphagia/vomiting, weight regain and insufficient weight loss. Excess weight loss (\%EWL) after Re-VBG, CSG and CRYGB was, respectively, 45\%, 57\% and 72\%. Eighteen patients (11.8\%) reported postoperative complications and $27 \%$ reported long-term complaints.

\section{Conclusion}

In terms of additional weight loss, postoperative complaints and reintervention rate, Roux-en-Y gastric bypass seems feasible as a revision for a failed VBG. Furthermore, the high number of complications after VBG and complications due to revisional procedures underline that VBG should be excluded as a primary option in bariatric surgery. 


\section{INTRODUCTION}

Obesity is a growing global problem, associated with morbidity, health care costs and even an increased mortality rate. ${ }^{1}$ For the treatment of obesity, bariatric surgery is very effective in achieving significantly more long-term weight loss and an improved lifestyle compared with conventional therapy. ${ }^{2,3}$ In 2011, over 340,000 bariatric procedures were performed worldwide. Among those procedures, around 2,300 procedures were a vertical banded gastroplasty (VBG), first described by Mason and later altered by MacLean et al. ${ }^{4-6}$ Aim of this procedure was to establish a restriction on food intake with a small stomach pouch, without compromising passage of food through the entire gastro-intestinal tract and thereby avoiding malabsorption of nutrients and medication. ${ }^{7}$ This procedure has shown in earlier reports to have good shortterm results in terms of weight loss and reduction in comorbidities. ${ }^{8-11}$ However, there are many studies reporting on the poor long-term results after VBG, showing a tendency for weight regain and other complications resulting in a high revision rate. ${ }^{12-14}$ Various options are available for revisional surgery after VBG, such as revision of the VBG (Re-VBG), conversion to sleeve gastrectomy (CSG) and conversion to Roux-en-Y gastric bypass (cRYGB), in which re-VBG appears to have the poorest outcome and CRYGB has the best short- and longterm results. ${ }^{15-19}$ However, data on the comparison between the revisional options remains scarce.

Although VBG had been abandoned some years ago in the Netherlands, still a number of patients can be expected to return with complaints after VBG. In the current series, all three mentioned options for revision have been performed. The aim of this study is to compare the outcome after these revisional procedures.

\section{MATERIALS AND METHODS}

\section{Methods}

This is a single-center retrospective study. A total of 392 patients underwent primary VBG, between January 1998 and December 2008. Since 2009, VBG was not performed anymore. Only patients undergoing primary VBG at the current center were included to reduce heterogeneity. Medical charts as well as additional postal questionnaires were reviewed. Included parameters were patient's characteristics, operative details of primary and secondary procedures, evolution of weight and comorbidities following both operations, findings at 
additional imaging, reason for revision, short-term complications and longterm complaints after revisional surgery.

The postal questionnaire contained questions on weight and comorbidities, on complaints dysphagia, vitamin deficiencies and incisional hernia. In case of insufficient weight loss, weight regain or complaints and without participation the follow-up program, the patient was invited to the outpatients department. In case of non-response, patients received a phone call and when there was no response at all, the data of the latest visit at the outpatient clinic were used as final outcome.

Excess weight was defined as the difference between the weight before surgery and the highest healthy weight, which is at a Body Mass Index (BMI) of $25 \mathrm{~kg} / \mathrm{m}^{2}$. Total excess weight loss (\%EWL) was defined as a percentage of the amount of excess weight lost after surgery, as described by Deitel et al. ${ }^{20}$ The weight before the primary VBG was used as baseline value to calculate \%EWL.

Weight loss was categorized according to the criteria described by Reinhold et al. ${ }^{21}$ These criteria consider a bariatric procedure successful when a \%EWL of at least $50 \%$ is achieved. Furthermore, change in $\mathrm{BMI}$ and \% total body weight loss (TBWL) was calculated. The evolution of any present comorbidities was categorized in stable, improved (reduced amount of medication used and/or a lower setting of a Continuous Positive Airway Pressure-device (CPAP)), resolved (no treatment), worse and de novo.

\section{Treatment}

Before primary VBG, all patients underwent assessment at our outpatient clinic by a surgeon, a psychologist and a dietitian to consider whether or not they were qualified for a bariatric procedure according to the standard IFSO guidelines for bariatric surgery. There was no specific algorithm for choosing the operative technique if they were approved for a bariatric procedure. There was a tendency for the option of a gastric bypass in case of more comorbidities, otherwise a vertical banded gastroplasty was chosen at the time. All patients underwent Mason-MacLean VBG, a standard VBG first described by Mason et al with transection of the vertical staple line as described by MacLean. ${ }^{4,5}$

Follow-up for these patients consisted of one year guidance by a psychologist, dietician and surgeon. Thereafter, a GP continued care unless weight loss problems or complaints were an issue. In such case patients underwent an analysis by all three disciplines and/or by means of a stomach $\mathrm{x}$-ray and/or a gastroscopy. If considered eligible for revision the options were a re-VBG, cSG or CRYGB. 
The Re-VBG technique meant in essence one of the 2 following adjustments. If the pouch was too large, a reshaping of the pouch was performed. The other option was an adjustment of the primarily placed band at the end of the gastric pouch.

A CSG meant a division of the lower part of the stomach $6 \mathrm{~cm}$ from the pylorus up to the transgastric window to remove the gastric fundus and part of the corpus and antrum. ${ }^{22}$ All sleeve gastrectomies were performed using a 34-Fr intraluminal boogie and stapled by use of the Endo GIA ${ }^{\text {TM }}$ (Covidien, New Haven, CT, USA).

A CRYGB started with identification of the polytetrafluoroethylene (PTFE) band. Then the stomach was transected horizontally at the proximal side of the band. The band was removed in most cases. The pouch was resized with use of the endoscopic stapler up to the angle of His. Then, an end-to-side gastrojejunostomy was constructed by a linear stapler and closed using Polysorb ${ }^{\text {TM }}$ sutures before 2009 and V-Loc ${ }^{\text {TM }}$ sutures after 2009 (Covidien, Mansfield, MA, USA). The alimentary limb, measuring 150-180 cm, was pulled up in an antecolic position. Finally, a side-to-side jejuno-jejunostomy was constructed, also using a linear stapler and closing the defect again with either Polysorb ${ }^{\text {TM }}$ or $\mathrm{V}-\mathrm{loc}^{\mathrm{T} M}$ sutures. Mostly, the procedure was finished by closing the mesenteric defects.

\section{Statistical analysis}

All data were collected retrospectively. Management and analysis as performed by using SPSS version 22, for Windows (SPSS Inc, Chicago, IL). Quantitative data are denoted as mean +/- standard deviation, whereas rates of complications and evolution on comorbidities are presented as a percentage. The student $t$-test, linear regression analysis and logistic regression analysis were used to determine any significance of the observed differences among subgroups. Statistical significance was identified when the $p$ value was less than 0.05 . An Odds Ratio (OR) was provided when applicable and considered significant when $\mathrm{OR}(95 \% \mathrm{Cl}) \neq 1$. Summative figures and tables were used when necessary.

No ethical approval was required for this study.

\section{RESULTS}

392 patients who underwent primary VBG were identified. According to the medical charts and questionnaires a total of 152 revisional procedures (38.7\%) were performed between April 1999 and June 2014, of which six patients 
underwent revision in another hospital. Necessary data of these patients was retrieved. Furthermore, these six patients did complete the postal questionnaire, so they were included in the analysis, together with the rest of the study population. Baseline characteristics are shown in Table 4.1.

Table 4.1 Baseline characteristics $(n=152)$.

\begin{tabular}{|c|c|c|c|c|}
\hline & $\begin{array}{c}\text { Re-VBG } \\
\mathrm{n}=21(13.8 \%) \\
\text { Mean } \pm \text { SD }\end{array}$ & $\begin{array}{c}\text { cSG } \\
\mathrm{n}=16(10.5 \%) \\
\text { Mean } \pm \text { SD }\end{array}$ & $\begin{array}{c}\text { cRYGB } \\
\text { n=115 (75.7\%) } \\
\text { Mean } \pm \text { SD }\end{array}$ & $p$-value \\
\hline Age (years) & $42.3 \pm 8.6$ & $41.6 \pm 11.4$ & $43.0 \pm 8.9$ & 0.828 \\
\hline Male : Female & $5: 16$ & $3: 13$ & $19: 96$ & 0.674 \\
\hline Body Mass Index before VBG $\left(\mathrm{kg} / \mathrm{m}^{2}\right)$ & $42.6 \pm 5.4$ & $43.6 \pm 5.0$ & $44.1 \pm 4.9$ & 0.445 \\
\hline \multicolumn{5}{|l|}{ Preoperative comorbidities } \\
\hline Type 2 Diabetes Mellitus (n) & 4 & 2 & 13 & 0.538 \\
\hline Hypertension (n) & 4 & 2 & 25 & 0.790 \\
\hline Dyslipidemia (n) & 0 & 3 & 11 & 0.111 \\
\hline Sleep apnea $(n)$ & 1 & 1 & 2 & 0.249 \\
\hline Osteo-articular disease (n) & 5 & 0 & 9 & 0.038 \\
\hline Patients with 1 or more comorbidity & 10 & 5 & 41 & 0.512 \\
\hline Operative time (min) & $77.0 \pm 39.2$ & $100.6 \pm 19.6$ & $130.7 \pm 47.3$ & $<0.001$ \\
\hline Length of hospital stay (days) & $3.1 \pm 2.9$ & $3.8 \pm 2.2$ & $4.1 \pm 5.8$ & 0.761 \\
\hline $\begin{array}{l}\text { Interval between VBG \& Revision } \\
\text { (months) }\end{array}$ & $12.3 \pm 10.7$ & $30.7 \pm 26.5$ & $47.8 \pm 34.8$ & $<0.001$ \\
\hline Average \%EWL after VBG (\%) & $61.7 \pm 27.0$ & $38.7 \pm 22.9$ & $43.5 \pm 25.0$ & 0.007 \\
\hline
\end{tabular}

Re-VBG: revision of the vertical banded gastroplasty; cSG: conversion to sleeve gastrectomy; cRYGB: conversion to Roux-en-Y gastric bypass; \%EWL: percentage of excess weight loss; SD: standard deviation.

The initial 392 patients showed an average \%EWL of $51.2 \pm 27.4 \%$ and $54 \%$ of all known comorbidities were either improved or resolved. The resolved comorbidities were not taken into account in the current study. The patients necessitating revision showed a significantly lower \%EWL of $45.4 \pm 25.8 \%$, compared to those not necessitating revision $(54.9 \pm 27.7 \%, p=0.001)$. At last follow-up, $58.4 \%(n=229)$ of the total of 392 patients reported long-term complaints, which in 152 patients led to a revisional procedure.

82.2\% of the current study population was female. Follow-up of patients necessitating second revision was taken into account until second revision. $A$ total of 127 patients (83.6\%) successfully completed last follow-up by either returning the postal questionnaire or answering the questions on the phone. This resulted in a mean follow-up after revisional surgery of $56.5 \pm 37.9$ months. In total, 25 patients did not return the postal questionnaire and could not be reached despite repeated attempts. Of these 25 patients, two patients deceased during follow-up due to a cause unrelated to bariatric surgery. Of these patients, the unreturned questionnaires were considered as missing data and 
the data of last known follow-up was used as final outcome so patients could be included in the analysis.

\section{Reasons for revision}

Complaints leading to revisional surgery are shown in Table 4.2. Six patients have had their revisional procedure in another center and therefore the complaints remained unknown. A possible surgically technical cause for failure of the VBG was found in $54.2 \%$ of all patients in this study.

Table 4.2 Complaints before revision $(n=152)$.

\begin{tabular}{lccccc}
\hline & $\begin{array}{c}\text { Re-VBG } \\
\mathbf{n = 2 1} \\
(\mathbf{n})\end{array}$ & $\begin{array}{c}\text { cSG } \\
\mathbf{n = 1 6} \\
\mathbf{( n )}\end{array}$ & $\begin{array}{c}\text { cRYGB } \\
\mathbf{n = 1 1 5} \\
\mathbf{( n )}\end{array}$ & Total (\%) & $\boldsymbol{p}$-value \\
\hline Vomiting/Dysphagia/Food intolerance & 17 & 8 & 36 & 40.2 & $<\mathbf{0 . 0 0 1}$ \\
Weight regain & 1 & 4 & 42 & 30.8 & $\mathbf{0 . 0 0 7}$ \\
Insufficient weight loss & 3 & 4 & 25 & 21.1 & 0.665 \\
Unknown & 0 & 0 & 6 & 3.9 & 0.792 \\
Severe GERD & 0 & 0 & 4 & 2.6 & 1.000 \\
Decline comorbidities & 0 & 0 & 1 & 0.7 & 1.000 \\
Excessive weight loss & 0 & 0 & 1 & 0.7 & 1.000 \\
\hline
\end{tabular}

Re-VBG: revision of the vertical banded gastroplasty; CSG: conversion to sleeve gastrectomy; CRYGB: conversion to Roux-en-Y gastric bypass; GERD: Gastro esophageal reflux disease.

Additional tests, in this study a stomach x-ray and/or a gastroscopy, were performed for additional analysis when necessary. The three main technical problems in this study population were a wide outlet, allowing faster passage of food through the pouch (17.1\%), pouch dilatation (15.8\%) and outlet stenosis (9.9\%). Other technical reasons for failure were band erosion (5.3\%), band luxation (displacement of the PTFE-band from its original position) (2.0\%), staple line dehiscence resulting in a fistula $(2.7 \%)$, pouch rotation $(0.7 \%)$ and band dehiscence $(0.7 \%)$.

\section{Intra- and postoperative complications}

126 procedures (82.9\%) were performed laparoscopically, 15 procedures (9.9\%) had a primary open approach and 11 (7.2\%) procedures were converted from a laparoscopic to an open approach. One conversion was due to an intraoperative gastro-intestinal perforation which could not be managed laparoscopically, the other procedures were converted because of an unacceptable laparoscopic overview due to extensive intra-abdominal adhesions. Only 2 intra-operative complications (1.4\%) occurred during surgery, both being an iatrogenic gastro-intestinal perforation. 
Complications in the 30-day postoperative period were seen in a combined total of 18 patients (11.8\%). No complications were seen after revision of the primary VBG (0/21). After CSG, three complications were objectified $(3 / 16=$ 18.8\%): one pneumonia, one patient suffering from persistent vomiting after surgery causing dehydration. No evident cause was found for the persistent vomiting. The third patient had an ileus. In the group of patients who underwent CRYGB, 15 complications were registered (15/115 = 13.0\%). Reoperation was necessary in two out of three patients with bleeding and in all patients with anastomotic leakage $(n=3)$. All leakages were found at the gastro-jejunostomy. Other complications included intra-abdominal abscesses $(n=3)$, wound infection $(n=2)$, pneumonia $(n=1)$, urinary tract infection $(n=$ $1)$, ileus $(n=1)$ and deep venous thrombosis $(n=1)$. The intra-abdominal abscesses all necessitated re-admission to the hospital for intravenous antibiotic treatment combined with either CT- or ultrasound-guided drainage. In total, eight patients were admitted for appropriate treatment of the complication, three patients did not necessitate readmission and seven complications occurred during primary admission. No significant difference was found in the total number of complications between the groups.

\section{Weight loss \& evolution of comorbidities}

When not including the follow-up after any secondary revisional procedure, the mean total \%EWL at last follow-up after primary revisional surgery was $66.4 \pm 25.8 \%$. In terms of change in $\mathrm{BMI}$, this meant an average reduction of $12.5 \pm 5.6 \mathrm{~kg} / \mathrm{m}^{2}$. Mean TBWL was $28.1 \pm 11.2 \%$. When including the 16 patients that underwent a second revisional procedure, \%EWL was $68.2 \pm 26.4 \%$. Change in BMI was $12.7 \pm 5.4 \mathrm{~kg} / \mathrm{m}^{2}$ and TBWL was $28.7 \pm 11.1 \%$.

At baseline, a total of 82 comorbidities were found amongst 56 patients. The separate improvement/resolution percentages for the three different procedures were $71.4 \%, 77.8 \%$ and $67.8 \%$ for respectively Re-VBG, cSG and CRYGB when considering each comorbidity as a separate entity. Figure 4.1 shows the improvement/resolution rates divided between the three groups. Table 4.3 shows the results after primary revisional surgery, stratified for each procedure.

\section{Long-term complaints}

At last follow-up after revisional surgery, 41 patients (27.0\%) reported complaints, which in 16 cases necessitated a second revisional procedure. All long-term complaints are displayed in Table 4.4. In one patient after Re-VBG, DVFI was caused by band erosion. 


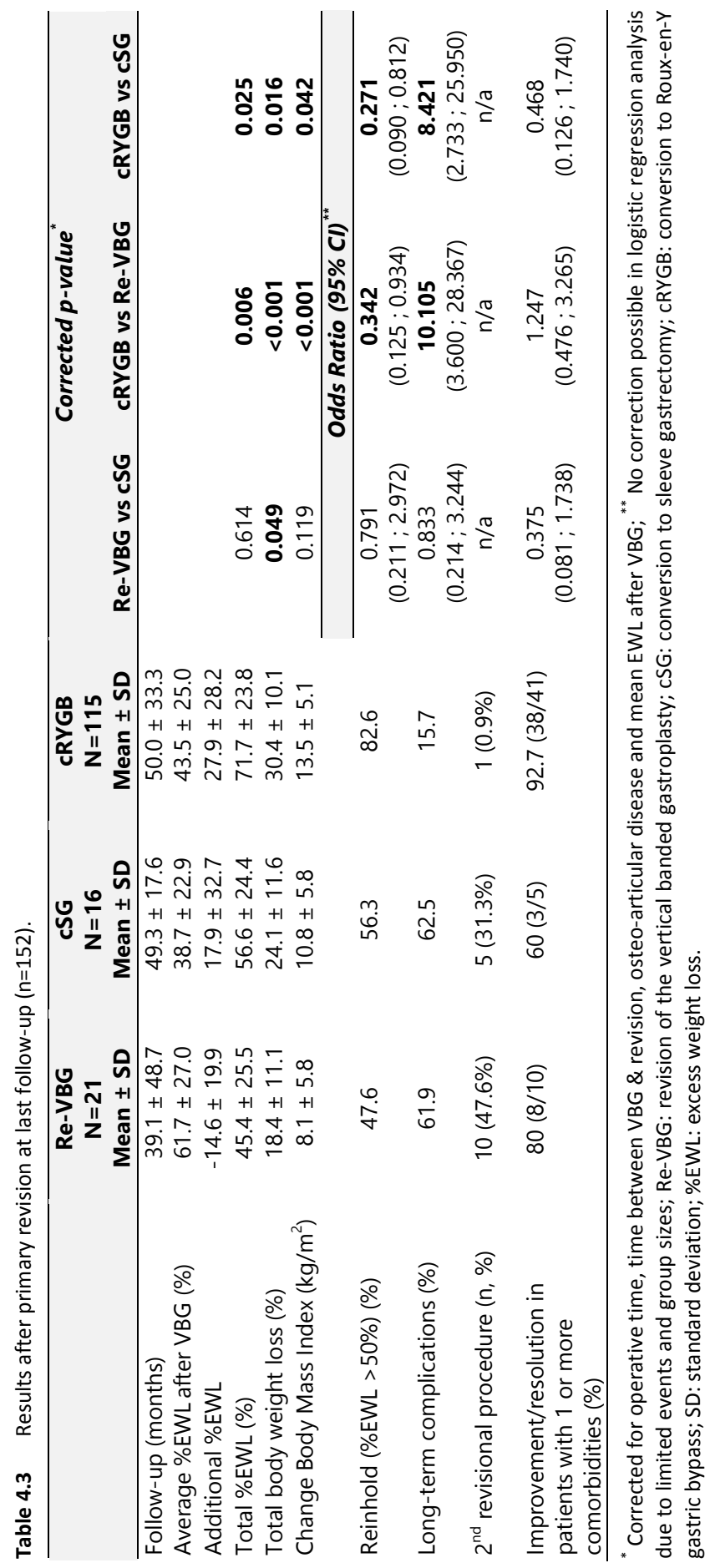




\section{Improvement/Resolution rates}

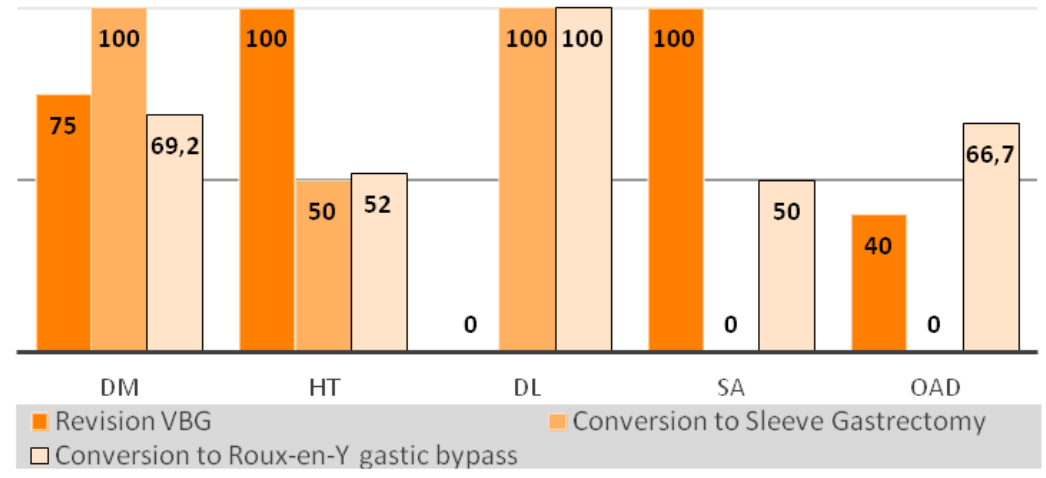

Figure 4.1 Improvement /resolution rates comorbidities. DM: type 2 diabetes mellitus; HT: hypertension; DL: dyslipidemia; SA: sleep apnea; OAD: osteo-articular disease; VBG: vertical banded gastroplasty.

Table 4.4 Long-term complaints after revision $(n=152)$.

\begin{tabular}{lcccc}
\hline & $\begin{array}{c}\text { Re-VBG } \\
\mathbf{n = 2 1} \\
(\mathbf{n})\end{array}$ & $\begin{array}{c}\text { cSG } \\
\mathbf{n = 1 6} \\
(\mathbf{n})\end{array}$ & $\begin{array}{c}\text { cRYGB } \\
\mathbf{n = 1 1 5} \\
\text { (n) }\end{array}$ & Total (\%) \\
\hline Vomiting/Dysphagia/Food intolerance & 4 & 4 & 6 & \\
Weight regain & 6 & 4 & 4 & 9.2 \\
Insufficient weight loss & 2 & 1 & 0 & 9.2 \\
Petersen's hernia & $\mathrm{n} / \mathrm{a}$ & $\mathrm{n} / \mathrm{a}$ & 4 & 2.0 \\
Incisional hernia & 0 & 0 & 3 & 2.6 \\
Recurrent abdominal pain & 1 & 1 & 1 & 2.0 \\
None & 8 & 6 & 97 & 73.0 \\
\hline
\end{tabular}

Re-VBG: revision of the vertical banded gastroplasty; CSG: conversion to sleeve gastrectomy; CRYGB: conversion to Roux-en-Y gastric bypass.

\section{Subgroups based on reason for revision}

Since the reason for revision may affect the outcome of the total weight loss, the evolution of comorbidities and potentially also the early postoperative course, additional analysis was performed. Patients undergoing revision for either weight regain or insufficient weight loss (WR/IWL) were compared to the other reasons given earlier in this manuscript. Results are shown in Table 4.5. 
Table 4.5 Subgroup analysis ( $n=152)$ (WR / IWL vs. Other complaints).

\begin{tabular}{|c|c|c|c|}
\hline & $\begin{array}{c}\text { WR / IWL } \\
n=79 \\
\text { Mean } \pm \text { SD }\end{array}$ & $\begin{array}{c}\text { Other } \\
n=73 \\
\text { Mean } \pm \text { SD }\end{array}$ & $p$-value \\
\hline Age (years) & $41.6 \pm 7.4$ & $43.9 \pm 10.7$ & 0.121 \\
\hline Male : Female $(n)$ & $13: 66$ & $14: 59$ & 0.661 \\
\hline Body Mass Index before VBG $\left(\mathrm{kg} / \mathrm{m}^{2}\right)$ & $44.7 \pm 5.0$ & $42.8 \pm 4.8$ & 0.016 \\
\hline Operative time $(\mathrm{min})$ & $128.2 \pm 46.3$ & $109.7 \pm 48.6$ & 0.020 \\
\hline Length of hospital stay (days) & $4.3 \pm 6.8$ & $3.4 \pm 2.0$ & 0.858 \\
\hline \multicolumn{4}{|l|}{ Type of revision } \\
\hline Re-VBG & 4 & 17 & \\
\hline cSG & 8 & 8 & 0.004 \\
\hline CRYGB & 67 & 48 & \\
\hline Average \%EWL after VBG (\%) & $31.3 \pm 19.0$ & $61.2 \pm 23.1$ & $<0.001$ \\
\hline Postoperative complications <30 days $(n, \%)$ & $12(15.2 \%)$ & $6(8.2 \%)$ & 0.184 \\
\hline Total \%EWL (\%) & $67.5 \pm 23.7$ & $65.2 \pm 28.1$ & 0.583 \\
\hline Reinhold (\%EWL > 50\%) (\%) & 79.7 & 74.0 & 0.398 \\
\hline Long-term complications (\%) & 22.8 & 28.8 & 0.399 \\
\hline $2^{\text {nd }}$ revisional procedure $(n)$ & 5 & 11 & 0.079 \\
\hline \multicolumn{4}{|l|}{ Improvement/Resolution rate $(\%, \mathrm{n})$} \\
\hline Type 2 Diabetes Mellitus & $90(9 / 10)$ & $55.6(5 / 9)$ & $\mathrm{n} / \mathrm{a}$ \\
\hline Hypertension & $76.9(10 / 13)$ & $44.4(8 / 18)$ & $\mathrm{n} / \mathrm{a}$ \\
\hline Dyslipidemia & $100(9 / 9)$ & $100(5 / 5)$ & $\mathrm{n} / \mathrm{a}$ \\
\hline Sleep apnea & $0(0 / 1)$ & $66.7(2 / 3)$ & $\mathrm{n} / \mathrm{a}$ \\
\hline Osteo-articular disease & $57.1(4 / 7)$ & $57.1(4 / 7)$ & $\mathrm{n} / \mathrm{a}$ \\
\hline
\end{tabular}

WR: weight regain; IWL: insufficient weight loss; \%EWL: excess weight loss; VBG: vertical banded gastroplasty; Re-VBG: revision of the vertical banded gastroplasty; CSG: conversion to sleeve gastrectomy; CRYGB: conversion to Roux-en-Y gastric bypass.

\section{Second revisional procedures}

A total number of 16 patients underwent a second revisional procedure. 10 patients underwent conversion from a revised VBG to RYGB, five patients had their sleeve converted to RYGB. One patient necessitated revision due to persistent vomiting after RYGB. Additional analysis showed a stenosis of the gastrojejunostomy. The most common reasons for second revision were weight regain (43.7\%) and DVFI (31.3\%). Other reasons were insufficient weight loss $(18.7 \%)$ and band erosion (6.3\%).

\section{DISCUSSION}

The absolute number of performed bariatric procedures is still increasing and therefore the number of revisional procedures can be expected to rise as well. Combined with the known poor long-term outcome after VBG, this fact strengthens the belief that more revisional procedures of failed VBG can be 
expected in the future. This study is the first to report on the comparison between Re-VBG, cSG and CRYGB after failed primary VBG.

The revision rate of VBG was almost 39\% (152 patients out of a total of 392 primary VBGs). The average \%EWL after failed VBG was $45.4 \pm 25.8 \%$ at last follow-up before revision. Patients who underwent Re-VBG had a noticeable better \%EWL after VBG at baseline. This can be explained by the much shorter average interval of only 12 months between the VBG and the revision, making follow-up too short to start noticing weight regain, a common reason for revision. $^{17}$

The main reasons for revision overall were similar to many other studies assessing either the long-term follow-up after VBG or the results after revision of the failed VBG. ${ }^{12,15,17,19,23}$ However, there is a difference in the type of complaints leading to the different revisional procedures in this study. Furthermore, the number of procedures differed between the groups in this study. These facts can be explained by various reasons. First of all, the indication for Re-VBG was limited (mainly band-related problems). In the early years, when a patient had complaints of DVFI, a Re-VBG was performed, especially when the DVFI was caused by band erosion. As more reports became available over the years, showing that $C R Y G B$ is a better revisional option than Re-VBG, that latter procedure was abandoned at an early stage and CRYGB has proven to be a better option and has been for quite some years, explaining the low number of VBGs. ${ }^{15,16,18}$ The last Re-VBG was performed in 2006. The second group, representing the $\mathrm{CSGs}$, appears to have a more similar pattern of reasons for revision as seen in the CRYGB group, compared to the Re-VBG group. The size of the cSG group however is small, mainly due to early abandonment of this procedure, because there are very limited reports on the outcome of CSG over the last years and the larger experience with CRYGB, which had already proven to be a reliable procedure. ${ }^{16,17,22}$ The last CSG after VBG was performed in January 2010. Since then, all revisional procedures after failed VBG were $C R Y G B$.

The results in this study show that, although no early postoperative complications were seen in this group and the improvement/resolution rate of comorbidities is comparable with the other groups, Re-VBG is not the preferred revisional procedure after failed primary VBG. The reasons are a low total $\% E W L$, high long-term complication rate and a high revision rate at long-term follow-up. Considering \%EWL, this study actually showed an average decrease after Re-VBG, resulting in patients regaining nearly $15 \%$ of their initial excess weight. This result may be biased by the already available experience that CRYGB appeared superior to Re-VBG and the limited indication for Re-VBG. ${ }^{18}$

The second group in this study were the cSGs. The long-term results after cSG are acceptable, with a significant better additional excess weight loss compared 
to Re-VBG and an improvement/resolution rate of comorbidities comparable with $C R Y G B$. Although CSG appears to give a lower chance on postoperative complications compared to $C R Y G B$, a significant higher long-term complication rate compared to $C R Y G B$ and a high second revision rate are showing the limits of this revisional procedure after failed primary VBG.

Although cSG appears to be superior compared to Re-VBG, this study confirms that $C R Y G B$ seems to be the best option of these three procedures. At last follow-up, patients showed an average \%EWL of almost $72 \%$, improvement or even resolution of comorbidities in $92.8 \%$ patients familiar with one or more obesity-related comorbidities. Furthermore, the chance of developing longterm complications after CRYGB is significantly lower compared to the other two revisional procedures. In contrast of these good results, we noticed a high postoperative complication rate of $13.0 \%$ after CRYGB. However, this rate is comparable with many previously published results showing postoperative complication rates of $6.5-25 \% .{ }^{15,23-25}$ In terms of \%EWL, these results are comparable with previously reported data after both revisional RYGB for failed VBG as well as after primary RYGB. ${ }^{24-27}$ The current good results may be affected by the used alimentary limb length of $150-180 \mathrm{~cm}$, on the other hand, this seems unlikely, since previous studies have shown that a limb length of $150 \mathrm{~cm}$ did not produce a better \%EWL compared to a limb length of $75-100 \mathrm{~cm}^{28,29}$

Considering the subgroup analysis performed to differentiate between weight loss-related complaints and other complaints, an expected significant difference was found in terms of \%EWL. Furthermore, a difference was found in the number of different revisional procedures between the two groups, which can be explained by the earlier reported difference in reason for revision between procedures. This may also explain the difference in operative time, since a Re-VBG takes a significant shorter time than a CRYGB. After revisional surgery, no significant differences were found in terms of \%EWL, postoperative complications and number of long-term complications and number of performed $2^{\text {nd }}$ revisional procedures.

The reported high revision rate and previously published unfavorable results underline the limits of this old restrictive procedure. ${ }^{12,13}$ These rates also strengthen the expectation that a number of patients will necessitate revision in the future, since VBG is currently still performed as a primary procedure. ${ }^{6}$ Although revisional surgery seems feasible, the high number of complications after revision should be taken into account.

These results should be interpreted with caution due to a number of limitations in this study. First of all, the unequal distribution of groups, mainly due to a bias caused by the center's greater experience with $C R Y G B$, thereby explaining the small number of Re-VBGs and cSGs. Furthermore, the retrospective design 
limits the reliability of the reported outcome. Since not all patients responded to our question to update the last known follow-up, a number of long-term complaints might have been missed, as well as potential revisional procedures performed in other hospitals. Not only the distribution between groups is unequal, also total time of follow-up is unequal, since Re-VBGs were performed only in the early and CRYGBs are still performed nowadays. The mean follow-up appears to be similar, but it should be kept in mind that Re-VBG showed a higher second revision rate as opposed to CSG and CRYGB and follow-up of the primary revision was taken into account until second revision. Keeping these limitations in mind, this study still suggests that Roux-en-Y gastric bypass is the superior choice for revisional surgery after failed primary VBG due to a good long-term \%EWL, a high improvement rate of comorbidities, a low long-term complication rate and a low percentage of necessitated second revisional procedures.

In conclusion: In terms of additional weight loss, number of postoperative complaints and necessitation of a second revision, the Roux-en-Y gastric bypass seems feasible as a revision for a failed VBG. Furthermore, the high number of complications after VBG and complications due to revisional procedures underline that VBG should be excluded as a primary option in bariatric surgery and other restrictive should be considered instead. 


\section{REFERENCES}

1. Selassie $M$, Sinha AC. The epidemiology and aetiology of obesity: a global challenge. Best Pract Res Clin Anaesthesiol. 2011;25(1):1-9.

2. Sjostrom L. Bariatric surgery and reduction in morbidity and mortality: experiences from the SOS study. Int J Obes (Lond). 2008;32 Suppl 7:S93-7.

3. Sjostrom L, Lindroos AK, Peltonen M, Torgerson J, Bouchard C, Carlsson B, et al. Lifestyle, diabetes, and cardiovascular risk factors 10 years after bariatric surgery. N Engl J Med. 2004; 351(26):2683-93.

4. Mason EE. Vertical banded gastroplasty for obesity. Arch Surg. 1982;117(5):701-6.

5. MacLean LD, Rhode BM, Forse RA. A gastroplasty that avoids stapling in continuity. Surgery. 1993;113(4):380-8.

6. Buchwald H, Oien DM. Metabolic/bariatric surgery worldwide 2011. Obes Surg. 2013;23(4): 427-36.

7. Mason EE, Doherty C, Cullen JJ, Scott D, Rodriguez EM, Maher JW. Vertical gastroplasty: evolution of vertical banded gastroplasty. World J Surg. 1998;22(9):919-24.

8. Lin YC, Chou FF, Chen SM, Wu CH. Vertical banded gastroplasty: a simple, effective and safe surgery for morbid obesity. Chang Gung Med J. 2003;26(10):754-60.

9. Morino M, Toppino M, Bonnet G, Rosa R, Garrone C. Laparoscopic vertical banded gastroplasty for morbid obesity. Assessment of efficacy. Surg Endosc. 2002;16(11):1566-72.

10. Wang W, Yu PJ, Lee YC, Wei PL, Lee WJ. Laparoscopic vertical banded gastroplasty: 5-year results. Obes Surg. 2005;15(9):1299-303.

11. Bekheit M, Katri K, Salam WN, Ezzat T, El Kayal el S. Rejecting the demise of vertical-banded gastroplasty: a long-term single-institute experience. Obes Surg. 2013;23(10):1604-10.

12. Marsk R, Jonas E, Gartzios H, Stockeld D, Granstrom L, Freedman J. High revision rates after laparoscopic vertical banded gastroplasty. Surg Obes Relat Dis. 2009;5(1):94-8.

13. Miller K, Pump A, Hell E. Vertical banded gastroplasty versus adjustable gastric banding: prospective long-term follow-up study. Surg Obes Relat Dis. 2007;3(1):84-90.

14. Schouten R, Wiryasaputra DC, van Dielen FM, van Gemert WG, Greve JW. Long-term results of bariatric restrictive procedures: a prospective study. Obes Surg. 2010;20(12):1617-26.

15. Cariani S, Agostinelli L, Leuratti L, Giorgini E, Biondi P, Amenta E. Bariatric Revisionary Surgery for Failed or Complicated Vertical Banded Gastroplasty (VBG): Comparison of VBG Reoperation (re-VBG) versus Roux-en-Y Gastric Bypass-on-VBG (RYGB-on-VBG). J Obes. 2010;2010.

16. lannelli A, Schneck AS, Ragot E, Liagre A, Anduze Y, Msika S, et al. Laparoscopic sleeve gastrectomy as revisional procedure for failed gastric banding and vertical banded gastroplasty. Obes Surg. 2009;19(9):1216-20.

17. Suter M, Ralea S, Millo P, Alle JL. Laparoscopic Roux-en-Y Gastric bypass after failed vertical banded gastroplasty: a multicenter experience with 203 patients. Obes Surg. 2012;22(10): 1554-61.

18. van Gemert WG, van Wersch MM, Greve JW, Soeters PB. Revisional surgery after failed vertical banded gastroplasty: restoration of vertical banded gastroplasty or conversion to gastric bypass. Obes Surg. 1998;8(1):21-8.

19. Vasas $P$, Dillemans $B$, Van Cauwenberge $S$, De Visschere $M$, Vercauteren C. Short- and longterm outcomes of vertical banded gastroplasty converted to Roux-en-Y gastric bypass. Obes Surg. 2013;23(2):241-8.

20. Deitel $M$, Greenstein RJ. Recommendations for reporting weight loss. Obes Surg. 2003; 13(2):159-60.

21. Reinhold RB. Critical analysis of long term weight loss following gastric bypass. Surg Gynecol Obstet. 1982;155(3):385-94.

22. Berende CA, de Zoete JP, Smulders JF, Nienhuijs SW. Laparoscopic sleeve gastrectomy feasible for bariatric revision surgery. Obes Surg. 2012;22(2):330-4. 
23. Schouten R, van Dielen FM, van Gemert WG, Greve JW. Conversion of vertical banded gastroplasty to Roux-en-Y gastric bypass results in restoration of the positive effect on weight loss and co-morbidities: evaluation of 101 patients. Obes Surg. 2007;17(5):622-30.

24. Cordera F, Mai JL, Thompson GB, Sarr MG. Unsatisfactory weight loss after vertical banded gastroplasty: is conversion to Roux-en-Y gastric bypass successful? Surgery. 2004;136(4): 731-7.

25. Iannelli A, Amato D, Addeo P, Buratti MS, Damhan M, Ben Amor I, et al. Laparoscopic conversion of vertical banded gastroplasty (Mason MacLean) into Roux-en-Y gastric bypass. Obes Surg. 2008;18(1):43-6.

26. Edholm D, Naslund I, Anders Karlsson F, Rask E, Sundbom M. Twelve-year results for revisional gastric bypass after failed restrictive surgery in 131 patients. Surg Obes Relat Dis. 2014;10(1): 44-8.

27. Mognol P, Chosidow D, Marmuse JP. Roux-en-Y gastric bypass after failed vertical banded gastroplasty. Obes Surg. 2007;17(11):1431-4.

28. Choban PS, Flancbaum L. The effect of Roux limb lengths on outcome after Roux-en-Y gastric bypass: a prospective, randomized clinical trial. Obes Surg. 2002;12(4):540-5.

29. Stefanidis D, Kuwada TS, Gersin KS. The importance of the length of the limbs for gastric bypass patients--an evidence-based review. Obes Surg. 2011;21(1):119-24. 


\section{CHAPTER 5}

Conventional versus fast track anesthesia protocol in an unselected group of patients undergoing revisional bariatric surgery

M.R. van Wezenbeek

S. Pouwels M.P. Buise

J.F. Smulders

S.W. Nienhuijs

G. van Montfort

International Journal of Surgery Open. 2015;1:22-27 


\section{ABSTRACT}

Introduction

Fast track care has proven to be safe and effective in primary bariatric procedures. The number of more complex revisional procedures is expected to rise over the next years. The aim was to evaluate the potential benefits and safety of a fast-track protocol in an unselected group of patients undergoing Roux-en-Y Gastric Bypass (rRYGBP) as revision.

\section{Method}

For this retrospective study, all patients undergoing rRYGBP between January 2005 and December 2013 were included and categorized between conventional care (CC) and fast track care (FT). Patient characteristics, operative details and intra- and early postoperative complications $<30$ days were analysed.

Results

A total of 407 patients were included for analysis. 303 patients (74.4\%) received peri- and postoperative treatment according to the fast track protocol. Mean age of the study population was $44.0 \pm 8.9$ years; mean pre-primary procedure BMI was $45.7 \pm 7.0 \mathrm{~kg} / \mathrm{m}^{2}$. A total of 54 (13.3\%) postoperative complications were registered (CC $19.2 \%$ vs. FT $11.2 \% ; p=0.038$ ). Both operative time (CC $135.3 \pm 42.6$ minutes vs. FT $79.3 \pm 29.3$ minutes; $p<0.001)$ as well as hospital stay (CC $5.1 \pm 6.3$ days vs. FT $3.1 \pm 5.3$ days; $p<0.001$ ) were significantly shorter in the FT group. A multivariate analysis on postoperative complications showed that fast track was not predictive for the occurrence of complications (OR=0.853; 95\% Cl [0.403-1.804]; $p=0.677)$.

\section{Conclusion}

Fast track care appears to be safe and efficient for patients undergoing revisional Roux-en-Y gastric bypass, but postoperative outcome may be highly dependent on surgical experience. 


\section{INTRODUCTION}

Over the last years, a high number of bariatric procedures have been performed worldwide. ${ }^{1}$ Therefore, it is expected that the number of revisions will increase over the next years. High revision rates up to $50 \%$ are found after either adjustable gastric banding (AGB) or vertical banded gastroplasty (VBG). Reported revision rates after sleeve gastrectomy (SG) are lower, keeping in mind that proper long-term follow-up after SG is scarce. ${ }^{2-7}$

A frequently performed revision for these failed restrictive bariatric procedures is conversion to Roux-en-Y gastric bypass (RYGBP). ${ }^{5,6,8}$ In the early days of bariatric surgery, revisions were questioned for their safety and additional benefit. ${ }^{9}$ Nowadays, for a specific group of patients with either complications of the primary bariatric procedure or weight regain, revisional bariatric surgery can be beneficial.

Currently, the morbidity rate after revisional RYGBP is found to be similar compared to primary RYGBP. ${ }^{10}$ Due to the high demand of bariatric procedures, fast track care is becoming increasingly popular for bariatric surgery. It is known to increase the efficiency and thereby the productivity on a daily basis, without increasing the risk of postoperative complications. ${ }^{11,12}$

Since revisional bariatric surgery has become a lot safer over the last years, the demand for revisional bariatric surgery is expected to increase and the implementation of fast-track protocols in bariatric surgery is growing worldwide, the question is raised whether a fast track protocol would be safe to implement in revisional bariatric surgery.

This study aimed to evaluate the potential benefits and safety of a fast-track protocol in an unselected group of patients undergoing conversion to RYGBP bypass after VBG, AGB or SG.

\section{METHODS}

For this retrospective analysis, medical charts of all patients undergoing revisional bariatric surgery between January 2005 and December 2013 at the Obesity Centre of the Catharina Hospital Eindhoven, a national referral center for revisional bariatric surgery were reviewed.

This study has been approved by the Institutional Review Board of the Catharina Hospital and has been performed in accordance with the ethical standards as laid down in the 1964 Declaration of Helsinki. All patients undergoing revisional Roux-en-Y gastric bypass (rRYGBP) after either adjustable gastric banding (AGB), sleeve gastrectomy (SG) of vertical banded gastroplasty (VBG, either Mason or 
Mason-MacLean approach) were included. Other revisional procedures (such as conversion to sleeve gastrectomy) were excluded.

This study was conducted according to the Strengthening the Reporting of Observational studies in Epidemiology (STROBE) statement.

Before January 2011, all bariatric patients were treated according to the hospital's conventional care (CC) protocol. Since January 2011, all patients, including those submitted to revisional procedures, at the Catharina hospital undergo bariatric surgery according to the new center-adjusted fast track care (FT) protocol.

\section{Fast track versus conventional care anaesthesia}

Table 5.1 gives an overview of the FT and CC protocol. Furthermore, the CC protocol from this hospital has been described in detail before [13]. The screening and preoperative work-up program was identical for the FT and CC group.

Many differences are found between CC and FT. Premedication in the CC protocol consisted of acetaminophen $1000 \mathrm{mg}$ and diazepam $5 \mathrm{mg}$. No premedication was included in the FT protocol. In both groups, patients received thrombosis prophylaxis by 5000 units of FraxiparinR (low-molecular weight heparin (LMWH)) and compression stockings. In both protocols, patients received standard hemodynamic monitoring (electrocardiography, pulsoximetry and non-invasive blood pressure measurement). In both groups, bispectral index monitoring and neuromuscular monitoring were routinely used to diminish the chance of awareness in the totally paralyzed patient and to antagonize the muscle relaxant properly. Medication used for induction of the anaesthesia and for perioperative anesthetic maintenance and postoperative analgesia are shown in Table 5.1.

At the end of the procedure, after antagonizing muscle relaxation with sugammadex, patients from the CC group were transferred from the operating room (OR) table to a normal ward bed by the OR personnel. In the FT protocol, desflurane and remifentanil were discontinued upon notification from the surgeon so the patients could be extubated immediately after the procedure and thus were able to move from the operation table to their bed themselves. Following to the CC protocol, all male patients with a Body Mass Index (BMI) over $45 \mathrm{~kg} / \mathrm{m}^{2}$ and all female patients with a BMl over $50 \mathrm{~kg} / \mathrm{m}^{2}$ were transferred to the intensive care unit (ICU) for respiratory monitoring due to a higher risk developing atelectasis, respiratory dysfunction and complications in combination with the postoperative use of morphine. In the FT group, all patients were admitted to the recovery and transferred to the short-stay surgical ward after 1-2 hours. Furthermore, in the FT group, revisional 
procedures were normally performed during regular bariatric programs between primary bariatric procedures, whereas in the CC group, revisional patients were planned on a separate operating program.

Table 5.1 Anaesthesia protocols.

\begin{tabular}{|c|c|}
\hline \multirow{2}{*}{$\begin{array}{l}\text { Conventional Care } \\
\text { Premedication }\end{array}$} & Fast Track Care \\
\hline & Premedication \\
\hline Acetaminophen 1000mg & None \\
\hline \multicolumn{2}{|l|}{ Diazepam 5mg } \\
\hline Thrombosis prophylaxis & Thrombosis prophylaxis \\
\hline Low molecular weight heparins (5000 IU) & Low molecular weight heparins (5000 IU) \\
\hline \multicolumn{2}{|l|}{ Compressed stockings } \\
\hline Induction & Induction \\
\hline Sufentanil 0.2-0.7 $\mu \mathrm{g} / \mathrm{kg}$ & Piritramide $0.2-0.3 \mathrm{mg} / \mathrm{kg}$ \\
\hline Propofol $2 \mathrm{mg} / \mathrm{kg}$ & Propofol $2 \mathrm{mg} / \mathrm{kg}$ \\
\hline \multirow[t]{3}{*}{ Rocuronium $0.6 \mathrm{mg} / \mathrm{kg}$} & Suxamethonium $1.0-1.5 \mu \mathrm{g} / \mathrm{kg} / \mathrm{hr}$ \\
\hline & Granisetron $3 \mathrm{mg}$ \\
\hline & Dexamethasone $8 \mathrm{mg}$ \\
\hline Maintenance & Maintenance \\
\hline Remifentanil 5-15 $\mu \mathrm{g} / \mathrm{kg} / \mathrm{hr}$ & Remifentanil 5-15 $\mu \mathrm{g} / \mathrm{kg} / \mathrm{hr}$ \\
\hline Sevoflurane or propofol 2-10 mg/kg/hr & Desflurane (6.0\% vol.) \\
\hline Rocuronium $0.4 \mathrm{mg} / \mathrm{kg} / \mathrm{hr}$ & Bispectral index monitoring \\
\hline \multicolumn{2}{|l|}{ Bispectral index monitoring } \\
\hline Postoperative analgesia & Postoperative analgesia \\
\hline Patient controlled analgesia (PCA) & Parecoxib $40 \mathrm{mg}$ \\
\hline \multirow[t]{3}{*}{ with $1 \mathrm{mg}$ morphine and droperidol } & Acetaminophen 4dd 1000 mg \\
\hline & Tramadol 3dd $100 \mathrm{mg}$ \\
\hline & Piritramide $0.2-0.3 \mathrm{mg} / \mathrm{kg}$ when indicated \\
\hline Postoperative care & Postoperative care \\
\hline Intensive Care Unit (ICU) or & Surgical ward \\
\hline Surgical ward & \\
\hline \multirow{6}{*}{$\begin{array}{l}\text { Additional concerns } \\
\text { High labour for personnel (transfer of the } \\
\text { patient) } \\
\text { Liberal intravenous fluid administration }\end{array}$} & Additional concerns \\
\hline & Direct mobilization \\
\hline & Restricted intravenous fluid administration \\
\hline & $(\max .1 \mathrm{~L}$ \\
\hline & perioperative, max. $1 \mathrm{~L}$ postoperative) \\
\hline & Liberal oral fluid intake \\
\hline
\end{tabular}

\section{Surgical procedure}

The technique of the rRYGBP differed between the different primary procedures. The main difference is found in the preparation before the construction of the gastric pouch. After SG, no additional preparations were necessitated except any potential adhesiolysis.

In case of rRYGBP after AGB, the band had to be removed first and the created wrap had to be undone. This was performed in one procedure, unless there was band erosion present. In that case, the band was removed in a separate 
laparoscopic procedure and at least three months later, the rRYGBP was performed. In case of an earlier VBG, procedure was started with the identification of the band. After identification, the stomach was transected horizontally at the proximal side of the band. The VBG-pouch was then resized with use of an endoscopic stapler (Endo GIA ${ }^{\mathrm{TM}}$ (Covidien, New Haven, CT, USA)), up to the angle of His.

After the creation of the pouch, an end-to-side antegastric gastrojejunostomy was constructed by a linear stapler (EndoGIA ${ }^{\mathrm{TM}}$ (Covidien, New Haven, CT, USA)) and closed using a running suture. The alimentary limb measured an estimated 150-180 cm. The limb was pulled up in an antecolic position in all procedures. The procedure was finished by constructing the jejuno-jejunostomy, again by using a linear stapler and running suture. Mostly, the procedure was finished by closing the mesenteric defect. The alimentary limb length and the closure of the mesenteric defects were not taken into account for the current analysis.

\section{Outcome}

Patients were followed at the bariatric outpatient clinic, as part of our center's follow-up protocol. To assess the safety of fast track care in bariatric revisional surgery, the primary outcome measurements for this study were intra- and postoperative complications less than 30 days after surgery. Included postoperative complications were bleeding, anastomotic leakage, gastrointestinal perforation, various infectious complications (e.g. abscess, pneumonia, and wound infection), any major cardiovascular event and venous thromboembolisms. Additionally, all postoperative complications were categorized according to the Clavien-Dindo scale. ${ }^{14} \mathrm{~A}$ multivariate analysis was performed to find potential predictors for postoperative complications after rRYGBP. Other outcome measurements were length of hospital stay, duration of the surgical procedure and the rate of readmissions.

\section{Statistical analysis}

All data for the current study were prospectively collected and analysed retrospectively. Data management and analysis was performed using SPSS version 22 for Windows (IBM Corporation, Armonk, NY, USA). Continuous variables were presented as mean \pm standard deviation (SD). Complications and rate of readmissions are presented as a percentage.

Dependent on the distribution and type of variable, either student's $t$-test, Mann-Whitney U-test, Chi-square test or Fisher's exact test was used to determine any statistical significance between the observed differences among 
groups. Differences were considered significant in case of a $p$ value less than 0.05 .

Covariates showing a trend towards significance for developing a postoperative complication $(p<0.1)$ were considered for multivariable logistic regression analysis. Odds ratio $(\mathrm{OR})$ and $95 \%$ confidence intervals $(\mathrm{Cl})$ were provided when applicable and considered significant when OR $(95 \% \mathrm{Cl}) \neq 1$. No ethical approval was required for this study. The Institutional Review Board for this retrospective study granted permission.

\section{RESULTS}

Between January 2005 and December 2013, a total of 407 patients (84\% female) with a mean BMI of $37.6 \pm 6.6 \mathrm{~kg} / \mathrm{m}^{2}$ were included for analysis. A total of 112 VBGs (27.5\%), 174 AGBs (42.0\%) and 124 SGs (30.5\%) were converted to RYGBP. Mean age of the study population was $44.0 \pm 8.9$ years, the mean BMI before the primary bariatric procedure was $45.7 \pm 7.0 \mathrm{~kg} / \mathrm{m}^{2}$.

A total of $104(25.6 \%)$ patients were treated according to the CC protocol and 303 patients $(74.4 \%)$ were treated according to the FT protocol. Patient characteristics of the two groups are shown in Table 5.2. No missing data were reported in the current study.

Table 5.2 Patient characteristics $(n=407)$.

\begin{tabular}{lccc}
\hline & $\begin{array}{c}\text { CC } \\
\mathbf{n = 1 0 4}(\mathbf{2 5 . 6 \% )} \\
\text { Mean } \mathbf{\text { SD }}\end{array}$ & $\begin{array}{c}\text { FT } \\
\mathbf{n = 3 0 3}(\mathbf{7 4 . 4 \% )} \\
\text { Mean } \pm \text { SD }\end{array}$ & \\
\hline Age (years) & $41.9 \pm 8.9$ & $44.7 \pm 8.8$ & $\mathbf{0 . 0 0 7}$ \\
Male : Female (n) & $15: 89$ & $50: 253$ & 0.618 \\
Body Mass Index (BMI) $\left(\mathrm{kg} / \mathrm{m}^{2}\right)$ & $37.6 \pm 6.5$ & $37.6 \pm 6.6$ & 0.977 \\
BMI before previous bariatric procedure $\left(\mathrm{kg} / \mathrm{m}^{2}\right)$ & $45.6 \pm 7.0$ & $45.8 \pm 7.0$ & 0.732 \\
Pre-operative comorbidities (n, \%) & & & \\
$\quad$ Diabetes mellitus type 2 & $15(14.4 \%)$ & $40(13.2 \%)$ & 0.753 \\
Hypertension & $31(29.8 \%)$ & $77(25.4 \%)$ & 0.381 \\
Dyslipidaemia & $4(3.8 \%)$ & $38(12.5 \%)$ & $\mathbf{0 . 0 1 2}$ \\
Sleep apnoea & $3(2.9 \%)$ & $22(7.3 \%)$ & 0.109 \\
Osteo-articular disease & $4(3.8 \%)$ & $30(9.9 \%)$ & 0.054 \\
Type of previous bariatric procedure (n, \%) & $23(22.1 \%)$ & $148(48.8 \%)$ & \\
Adjustable Gastric Banding (AGB) & $26(25.0 \%)$ & $98(32.3 \%)$ & $<\mathbf{0 . 0 0 1}$ \\
Sleeve Gastrectomy (SG) & $55(52.9 \%)$ & $57(18.8 \%)$ & \\
Vertical Banded Gastroplasty (VBG) & & & \\
ASA classification (n, \%) & $8(7.7 \%)$ & $12(4.0 \%)$ & \\
I & $92(88.5 \%)$ & $274(90.4 \%)$ & 0.277 \\
II & $4(3.8 \%)$ & $17(5.6 \%)$ & \\
III & $20(19.2 \%)$ & $3(1.0 \%)$ & $<\mathbf{0 . 0 0 1}$ \\
Primary open approach (n, \%) & & & \\
\hline
\end{tabular}

CC: conventional care; FT: fast track care; SD: standard deviation. 
A total of 23 procedures employed an open approach and 384 procedures of laparoscopic approach. 11 procedures (2.8\%) were converted to an open approach during surgery (CC group 6/84 vs. FT group 5/300). Seven procedures were converted due to extensive adhesions of the previous procedure; three conversions were due to an intra-operative bleeding and one due to a large incisional hernia. All postoperative complications, including the stratification according to the Clavien-Dindo scale and the readmission rate are shown in Table 5.3.

Mean operating time was $135.3 \pm 42.6$ minutes in the CC group vs. $79.3 \pm 29.3$ minutes in the

FT group; $(p \leq 0.001)$ and the mean length of hospital stay was $5.1 \pm 6.3$ days in the CC group vs. $3.1 \pm 5.3$ days in the FT group; $(p \leq 0.001)$.

Following the criteria as described in the Methods section, a total of 54 patients (13.3\%) developed a postoperative complication $<30$ days after rRYGBP, of which 22 patients (5.4\%) necessitated a surgical reintervention (11 anastomotic leaks, four bleedings, four gastro-intestinal perforations and three intraabdominal abscesses).

Table 5.3 Peri- and postoperative outcome $(n=407)$.

\begin{tabular}{|c|c|c|c|}
\hline & $\begin{array}{c}\text { CC } \\
n=104(25.6 \%) \\
\text { Mean } \pm \text { SD }\end{array}$ & $\begin{array}{c}\text { FT } \\
\mathrm{n}=303(74.4 \%) \\
\text { Mean } \pm \text { SD }\end{array}$ & $p$-value \\
\hline Intra-operative complications (n, \%) & $5(4.8 \%)$ & $3(1.0 \%)$ & 0.029 \\
\hline Postoperative complications (n, \%) & $20(19.2 \%)$ & $34(11.2 \%)$ & 0.038 \\
\hline Surgical complications (n, \%) & $13(12.5 \%)$ & $19(6.3 \%)$ & 0.042 \\
\hline Anastomotic leakage & 8 (7.7\%) & $11(3.6 \%)$ & 0.106 \\
\hline Bleeding & $5(4.8 \%)$ & $5(1.7 \%)$ & 0.132 \\
\hline Gastro-intestinal perforation & $1(1.0 \%)$ & $3(1.0 \%)$ & 1.000 \\
\hline Infectious complications ( $\mathrm{n}, \%)$ & $11(10.6 \%)$ & $25(8.3 \%)$ & 0.471 \\
\hline Intra-abdominal abscess & 7 (6.7\%) & 19 (6.3\%) & 0.868 \\
\hline Pneumonia & $2(1.9 \%)$ & $6(2.0 \%)$ & 1.000 \\
\hline Urinary tract infection & $1(1.0 \%)$ & 0 & 0.256 \\
\hline Wound infection & $2(1.9 \%)$ & $3(1.0 \%)$ & 0.606 \\
\hline Cardiovascular complications (n, \%) & 0 & 0 & $\mathrm{n} / \mathrm{a}$ \\
\hline Venous thromboembolism & 0 & 0 & $\mathrm{n} / \mathrm{a}$ \\
\hline Readmissions (n, \%) & $10(9.6 \%)$ & $24(7.9 \%)$ & 0.590 \\
\hline \multicolumn{4}{|l|}{ Clavien-Dindo classification ( $\mathrm{n}, \%)$} \\
\hline Clavien-Dindo I & 0 & 0 & $\mathrm{n} / \mathrm{a}$ \\
\hline Clavien-Dindo II & $8(7.7 \%)$ & $8(2.6 \%)$ & 0.036 \\
\hline Clavien-Dindo III & $10(9.6 \%)$ & $25(8.3 \%)$ & 0.668 \\
\hline Clavien-Dindo IV & $2(1.9 \%)$ & $1(0.3 \%)$ & 0.162 \\
\hline Clavien-Dindo V & 0 & 0 & $\mathrm{n} / \mathrm{a}$ \\
\hline Serious adverse events (Clavien-Dindo $\geq 3 a)(n, \%)$ & $12(11.5 \%)$ & $26(8.6 \%)$ & 0.371 \\
\hline
\end{tabular}

CC: conventional care; FT: fast track care; SD: standard deviation 


\section{Multivariate analysis}

A multivariate analysis was conducted on the occurrence of postoperative complications after rRYGBP. Type 2 diabetes mellitus $(p=0.015)$, operative time $(p=0.005)$, an open procedure $(p<0.001)$, conversion to open procedure $(p=0.008)$, ASA risk classification $(p=0.055)$ and conventional care protocol $(p=0.038)$ were found to be significant factors $(p<0.1)$ for developing postoperative complications after revisional RYGBP in this study after univariate analysis. Although significant between groups, age, dyslipidemia and type of previous bariatric procedure were not found to be univariate significant predictors for the occurrence of postoperative complications $(p>0.1)$. After multivariate analysis, a primary open procedure was found to be a significant independent risk factor for postoperative complications $(\mathrm{OR}=9.155 ; 95 \% \mathrm{Cl}$ [3.064-27.359]; $p<0.001)$.

\section{DISCUSSION}

Fast track anaesthesia protocols have shown to be efficient in optimizing and standardizing perioperative care and result in a reduction in hospital stay and postoperative morbidity. Our study showed a decrease in hospital stay (from $5.1 \pm 6.3$ days in the CC group to $3.1 \pm 5.3$ days in the FT group) and a decrease in postoperative complications (from $19.2 \%$ in the CC group to $11.2 \%$ in the FT group). A primary open procedure was found to be an independent predictor for postoperative complications. Also operative time significantly decreased from $135.3 \pm 42.6$ minutes to $79.3 \pm 29.3$ minutes (CC and FT group, respectively).

Multiple studies have assessed the safety and efficiency of fast track care in both bariatric as well as in other abdominal procedures. ${ }^{11,12,15}$ Be that as it may, all fast track studies in a bariatric population focused on patients undergoing a primary bariatric procedure, leaving out the more complex and difficult revisional procedures. ${ }^{11,12,16,17}$ Therefore, this study assessed an unselected group of patients undergoing revisional RYGBP. Results may contribute to fast track implementation in revisional bariatric surgery. Potentially, current results may contribute to the consideration of a fast track protocol in other abdominal re-do procedures. The results show significant reduction in postoperative complications $<30$ days and less surgical complications since the implementation of the fast track protocol. Furthermore, less minor complications (Clavien-Dindo $\leq 2$ ) were observed in the FT group, and both operative time and hospital stay were significantly reduced. 
Few differences were found between the CC group and the FT group. Patients in the FT group were on average a few years older. Previous research has shown that patient with a higher age have an increased risk at postoperative complications. ${ }^{18}$ Nonetheless, age turned out not to be a significant predictor after univariate analysis on postoperative complications in the current study. The type of previous bariatric procedure differed between groups, which might be explained by the time lapse. VBG used to be a commonly performed procedure but was abandoned some years ago and replaced by the newer AGB and SG. ${ }^{19}$ The number of primary open procedures differed significantly between groups and has shown to be an independent factor in the occurrence of postoperative complications.

Noticeable is the significantly smaller number of Clavien-Dindo 1 or 2 complications. Although an ultrasound or CT-scan was not performed routinely, none of the patients suffered from a clinically obvious venous thromboembolism, which might suggest that stockings are not necessitated in these revisional procedures and low molecular weight heparins in the proper dose suffice. In current literature, there is still no consensus on the proper dose of low molecular weight heparins.

The postoperative complication rate significantly decreased from $19.2 \%$ in the CC group to $11.2 \%$ in the FT group. These rates are very comparable to previously published results on revisional RYGBP after failed VBG, AGB or SG. ${ }^{6,20-22}$ The complication rate of the FT group is even comparable with some studies on the outcome after primary RYGBP. ${ }^{23,24}$ Whether the reduction in rate of complication is caused purely by this implementation is arguable, as many factors may have influenced the occurrence of postoperative complications.

The choice for a primary open procedure has shown to be an independent predictive factor for the occurrence of complications in this study. Nevertheless, the total number of open procedures is still quite low, which makes it unlikely that this is the only explanation for the decrease in complications. Surgical experience should also be taken into account, as the CC group includes the first 100 revisional RYGBP procedures. Shikora et al. Previously showed a large reduction in complications between the first 100 patients and the following 650 patients undergoing primary RYGBP. ${ }^{23}$ This may be applicable in the current study as well, however, primary RYGBP has been performed for many years by experienced surgeons at this center and the surgical technique of a revisional RYGBP is essentially similar to the one of a primary RYGBP.

The use of fixed operating room teams during the day will have a contributing factor as well. ${ }^{25}$ These fixed teams were implemented at the same time as the use of the fast track protocol in this hospital. The truth on the reduction of the postoperative complication rate is most likely found somewhere in the middle, 
meaning that expertise, laparoscopic approach, fixed teams and fast track care are all likely to be a contributing factor.

The reduction in percentage minor complications (Clavien-Dindo $\leq 2$ ) may be caused by the fast track protocol, by promoting faster mobilization of patients, the decreased operating time and improved health care over the years.

The readmission rates in the CC group and the FT group were $9.6 \%$ and $7.9 \%$, respectively. These percentages are found to be similar to previous results in studies on fast track implementation in primary RYGBP surgery. ${ }^{12,16}$ Moreover, this study confirms the reduction in both operative time and length of hospital stay. Operative time decreased with a mean of more than 55 minutes, which can be explained by a combination of a reduced number of open procedures, increased surgical expertise and the use of fixed teams during the day. Fast

track care has shown to significantly reduce the length of hospital stay. ${ }^{12,26}$ This result can be confirmed by the current study, in which the mean hospital stay was reduced by 2 days, implying a substantial reduction in health care costs. ${ }^{27}$ Whether this solely caused by the implementation of fast track is arguable, since primary open approach (possibly resulting in a longer postoperative recovery) and a lower rate of complications during primary admission will have an influence on the average length of hospital stay.

\section{Limitations}

There are some limitations to the current study design, but this study showed that revisional bariatric procedures may benefit from a center-specific fast track protocol. First, the retrospective approach is limiting since results are dependent on the accuracy with which the medical charts were managed. Second, the design also limits the means to firmly objectify the predictive value of a learning curve in this study on revisional RYGBP. The combination of maturation of the surgical team, the high volume (currently still increasing) of revisional bariatric surgery in this center and the increased anesthesiological experience may bias the results of the fast track group. Drawback of the current study may be the fact that groups were not matched and that there were differences in performed operations during time. Our center is an expert center on revisional bariatric surgery; therefore our patient population is very diverse. This makes our patient population different from other centers. Our study did not stratify complications on their occurrence during primary admission or readmission, which might have been of clinical interest. Also our fast track anaesthesia protocol is center-adjusted and there might be few differences with other bariatric centers in terms of the used anesthetic agents.

Finally, our results might be biased by the course of time. As mentioned earlier, there is a combined influence of the implementation of the fast track 
anaesthesia protocol, the maturation of surgical experience and the use of fixed teams at the operating room. In our opinion, the effect of the surgical learning curve is very difficult to eliminate from our study results. Also the fact that the CC group and FT group differ in primary open procedures is a result of time and the emerging expert status of our center for revisional bariatric surgery. Despite these limitations and the fact that further research is needed to substantiate the current results, fast track care appears to be safe and efficient for patients undergoing revisional Roux-en- $Y$ gastric bypass and may be beneficial in other abdominal revisional procedures.

\section{Conclusion}

Fast track care appears to be safe and efficient for patients undergoing revisional Roux-en-Y gastric bypass, but postoperative outcome may be highly dependent on surgical experience. 


\section{REFERENCES}

1. Buchwald H, Oien DM. Metabolic/bariatric surgery worldwide 2011. Obes Surg. 2013;23(4): 427-36.

2. Baltasar A, Bou R, Arlandis F, Martinez R, Serra C, Bengochea M, et al. Vertical banded gastroplasty at more than 5 years. Obes Surg. 1998;8(1):29-34.

3. Gautier T, Sarcher T, Contival N, Le Roux $Y$, Alves A. Indications and mid-term results of conversion from sleeve gastrectomy to Roux-en-Y gastric bypass. Obes Surg. 2013;23(2): 212-5.

4. Miller K, Pump A, Hell E. Vertical banded gastroplasty versus adjustable gastric banding: prospective long-term follow-up study. Surg Obes Relat Dis. 2007;3(1):84-90.

5. van Gemert WG, van Wersch MM, Greve JW, Soeters PB. Revisional surgery after failed vertical banded gastroplasty: restoration of vertical banded gastroplasty or conversion to gastric bypass. Obes Surg. 1998;8(1):21-8.

6. van Rutte PW, Smulders JF, de Zoete JP, Nienhuijs SW. Indications and short-term outcomes of revisional surgery after failed or complicated sleeve gastrectomy. Obes Surg. 2012;22(12): 1903-8.

7. DeMaria EJ, Sugerman HJ, Meador JG, Doty JM, Kellum JM, Wolfe L, et al. High failure rate after laparoscopic adjustable silicone gastric banding for treatment of morbid obesity. Ann Surg. 2001;233(6):809-18.

8. Jennings NA, Boyle M, Mahawar K, Balupuri S, Small PK. Revisional laparoscopic Roux-en-Y gastric bypass following failed laparoscopic adjustable gastric banding. Obes Surg. 2013;23(7): 947-52.

9. Schwartz RW, Strodel WE, Simpson WS, Griffen WO, Jr. Gastric bypass revision: lessons learned from 920 cases. Surgery. 1988;104(4):806-12.

10. Deylgat B, D'Hondt M, Pottel H, Vansteenkiste F, Van Rooy F, Devriendt D. Indications, safety, and feasibility of conversion of failed bariatric surgery to Roux-en-Y gastric bypass: a retrospective comparative study with primary laparoscopic Roux-en-Y gastric bypass. Surg Endosc. 2012;26(7):1997-2002.

11. Dogan K, Kraaij L, Aarts EO, Koehestanie P, Hammink E, van Laarhoven CJ, et al. Fast-track bariatric surgery improves perioperative care and logistics compared to conventional care. Obes Surg. 2015;25(1):28-35.

12. Geubbels N, Bruin SC, Acherman YI, van de Laar AW, Hoen MB, de Brauw LM. Fast track care for gastric bypass patients decreases length of stay without increasing complications in an unselected patient cohort. Obes Surg. 2014;24(3):390-6.

13. van Rutte PW, Luyer MD, de Hingh IH, Nienhuijs SW. To Sleeve or NOT to Sleeve in Bariatric Surgery? ISRN surgery. 2012;2012:674042.

14. Dindo D, Demartines N, Clavien PA. Classification of surgical complications: a new proposal with evaluation in a cohort of 6336 patients and results of a survey. Ann Surg. 2004; 240(2):205-13.

15. Sosada K, Wiewiora M, Piecuch J, Zurawinski W. Fast track in large intestine surgery - review of randomized clinical trials. Wideochirurgia $\mathrm{i}$ inne techniki malo inwazyjne = Videosurgery and other miniinvasive techniques / kwartalnik pod patronatem Sekcji Wideochirurgii TChP oraz Sekcji Chirurgii Bariatrycznej TChP. 2013;8(1):1-7.

16. Dallal RM, Trang A. Analysis of perioperative outcomes, length of hospital stay, and readmission rate after gastric bypass. Surg Endosc. 2012;26(3):754-8.

17. Lemanu DP, Singh PP, Berridge K, Burr M, Birch C, Babor R, et al. Randomized clinical trial of enhanced recovery versus standard care after laparoscopic sleeve gastrectomy. Br J Surg. 2013;100(4):482-9.

18. Stenberg E, Szabo E, Agren G, Naslund E, Boman L, Bylund A, et al. Early complications after laparoscopic gastric bypass surgery: results from the Scandinavian Obesity Surgery Registry. Ann Surg. 2014;260(6):1040-7. 
19. Schouten R, Wiryasaputra DC, van Dielen FM, van Gemert WG, Greve JW. Long-term results of bariatric restrictive procedures: a prospective study. Obes Surg. 2010;20(12):1617-26.

20. Coblijn UK, Verveld CJ, van Wagensveld BA, Lagarde SM. Laparoscopic Roux-en-Y gastric bypass or laparoscopic sleeve gastrectomy as revisional procedure after adjustable gastric band--a systematic review. Obes Surg. 2013;23(11):1899-914.

21. Perathoner A, Zitt M, Lanthaler M, Pratschke J, Biebl M, Mittermair R. Long-term follow-up evaluation of revisional gastric bypass after failed adjustable gastric banding. Surg Endosc. 2013;27(11):4305-12.

22. Suter M, Ralea S, Millo P, Alle JL. Laparoscopic Roux-en-Y Gastric bypass after failed vertical banded gastroplasty: a multicenter experience with 203 patients. Obes Surg. 2012;22(10): 1554-61.

23. Shikora SA, Kim JJ, Tarnoff ME, Raskin E, Shore R. Laparoscopic Roux-en-Y gastric bypass: results and learning curve of a high-volume academic program. Arch Surg. 2005;140(4):362-7.

24. Suter M, Donadini A, Romy S, Demartines N, Giusti V. Laparoscopic Roux-en-Y gastric bypass: significant long-term weight loss, improvement of obesity-related comorbidities and quality of life. Ann Surg. 2011;254(2):267-73.

25. Stepaniak PS, Heij C, Buise MP, Mannaerts GH, Smulders JF, Nienhuijs SW. Bariatric surgery with operating room teams that stayed fixed during the day: a multicenter study analyzing the effects on patient outcomes, teamwork and safety climate, and procedure duration. Anesth Analg. 2012;115(6):1384-92.

26. Jacobsen HJ, Bergland A, Raeder J, Gislason HG. High-volume bariatric surgery in a single center: safety, quality, cost-efficacy and teaching aspects in 2,000 consecutive cases. Obes Surg. 2012;22(1):158-66.

27. Taheri PA, Butz DA, Greenfield L. Length of stay has minimal impact on the cost of hospital admission. J Am Coll Surg. 2000;191(2):123-30. 


\section{CHAPTER 6}

Conversion to gastric bypass after either failed gastric band or failed sleeve gastrectomy

M.R. van Wezenbeek T.R. van Oudheusden J.P.J.G.M. de Zoete J.F. Smulders

S.W. Nienhuijs Obes Surg. 2017;27(1):83-89

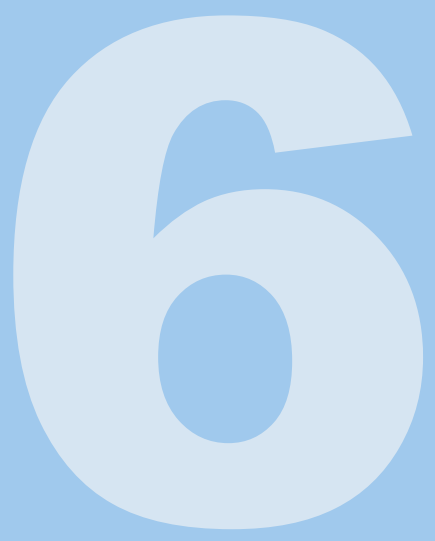




\section{ABSTRACT}

\section{Background}

Roux-en-Y gastric bypass (RYGB) is still considered the gold standard in bariatric surgery. Before, adjustable gastric banding (AGB) was regarded as an alternative, nowadays, sleeve gastrectomy (SG) is a more favorable alternative. In case of unsatisfactory results, RYGB is often performed as secondary procedure. Conversion of an AGB is associated with a high risk at complications, the hypothesis was that this would be less after conversion of an SG.

\section{Methods}

All patients undergoing conversion to RYGB after AGB or SG between 2005 and 2012 were included for retrospective analysis. Patient characteristics, operative details, postoperative complications, the relief of complaints, weight loss, reasons for failure and evolution of known comorbidities up to two years were analyzed.

\section{Results}

A total of 178 patients were included (79.8\% female): AGB 110 (61.8\%) vs. SG 68 (38.2\%). Main reasons for conversion were weight regain/insufficient weight loss (48.4\%) or dysphagia/reflux complaints (39.9\%). Surgical complications were found in 19 patients (AGB 13 vs. SG 6; $p=0.530$ ). Infectious complications occurred in 13 patients ( $A G B 11$ vs. SG 2; $p=0.135$ ). Total body weight loss was equal between groups after two years (AGB $31.6 \pm 11.0 \%$ vs. SG $31.6 \pm 12.0 \%$; $p=0.998)$. Similar results were found in a subgroup analysis on patients undergoing conversion for additional weight loss (AGB $31.7 \pm 11.7 \%$ vs. SG $27.0 \pm 13.1 \% ; p=0.173)$.

\section{Conclusions}

Conversion to RYGB after failed AGB or SG showed comparable short-term results in terms of postoperative complications and weight loss. 


\section{INTRODUCTION}

For the treatment of morbid obesity, Roux-en-Y gastric bypass (RYGB) has been considered the gold standard for many years. Not long ago, adjustable gastric banding ( $A G B$ ) was considered a feasible alternative as it showed a low postoperative complication rate and acceptable weight loss percentages. ${ }^{1}$ Drawback to this procedure are the reported high long-term complication rates, often necessitating removal of the band or conversion to RYGB. ${ }^{2,3}$ The more recently introduced sleeve gastrectomy (SG) poses a reliable alternative to both the $A G B$ and the RYGB, showing similar short- and mid-term results between RYGB and SG in terms of morbidity rate, weight loss and obesityrelated comorbidity improvement. ${ }^{4-6}$

The drawback of the SG can be similar to the AGB resulting into long-term complaints requiring a second bariatric procedure. Even though proper longterm studies after SG are not yet available, current literature reports a much lower re-do percentage after SG compared to $A G B .^{7-9}$

In case of failure necessitating a surgical reintervention of either AGB or SG, the most feasible option is currently still RYGB, however other options are emerging such as the mini gastric bypass, duodenal switch and the recently introduced single anastomosis duodeno-ileal bypass (SADI). ${ }^{9-14}$ Conversion of a failed AGB is risky, as multiple studies have shown postoperative complications rates of over $10 \% .{ }^{11,15,16}$ The new gastric pouch of the RYGB has to be created in the same area where the band was placed, which hypothetically can lead to more complications. Since this is not the case after SG, the complication rate may be lower after conversion of a failed SG to RYGB. Furthermore, data on bariatric re-do surgery after sleeve gastrectomy is currently still scarce. Therefore, the aim of this study was to compare the short-term-results after secondary RYGB for failed AGB versus failed SG.

\section{METHODS AND DESIGN}

All patients undergoing conversion to Roux-en-Y gastric bypass (CRYGB) between January $1^{\text {st }} 2007$ and December $31^{\text {st }} 2012$ were analyzed. For this study, all patients undergoing CRYGB after either failed primary adjustable gastric banding (AGB) or failed primary sleeve gastrectomy (SG) were included for analysis. A planned secondary procedure was regarded as insufficient weight loss.

Patient demographics, operative details, postoperative complications and follow-up until 2 years after surgery were included in the analysis. During 
follow-up postoperative weight and the evolution of any known obesity-related comorbidities were evaluated. The obesity-related comorbidities included type 2 diabetes mellitus, hypertension, dyslipidemia, sleep apnea and osteo-articular disease.

Primary outcome for this study were postoperative surgical complications $<30$ days. Anastomotic leakage, bleeding, incidental gastrointestinal perforation and intra-abdominal abscess were considered surgical complications.

Secondary outcomes were infectious complications such as pneumonia, urinary tract infection and wound infection were scored. Intra-abdominal abscesses were also taken into account for the infectious complications. All complications were stratified according to the Clavien-Dindo scale. ${ }^{17}$

Other secondary outcome measurements were additional weight loss and the evolution of any known obesity-related comorbidities. An additional analysis on weight loss and evolution of comorbidities was performed between the different reasons for the secondary procedure.

Weight loss was denoted as percentage excess weight loss (\%EWL), percentage of total body weight loss (\%TBWL) and change in body mass index (CBMI). The highest weight before the primary bariatric procedure was considered the starting weight for calculating weight loss. \%EWL was defined as percentage of excess weight lost after surgery, in which excess weight was defined as the difference between the weight before surgery and the highest healthy weight $\left(\mathrm{BMI}=25 \mathrm{~kg} / \mathrm{m}^{2}\right)^{18}$

The evolution of comorbidities was categorized as stable, improved, resolved, worse and de novo. Improved would indicate a reduced amount of medication, improved lab results and/or a lower setting of a Continuous Positive Airway Pressure-device (CPAP). Resolved meant no medication or treatment necessary and/or normalized lab results.

\section{Surgical treatment}

The CRYGB technically differed between patients, depending on the primary bariatric procedure. Main difference was found in the preparation for constructing the gastric pouch. After primary SG, except adhesiolysis, no additional preparations had to be made.

In case of primary AGB, the band had to be removed and the created wrap had to be undone prior to the creation of the new pouch. Until 2010, this was done in the same procedure as the Roux-en-Y gastric bypass. Since then, the band was removed during a separate laparoscopic procedure and around at least three months later, the CRYGB was performed. 
The gastric pouch was created by using an endoscopic stapler (EndoGIA ${ }^{\text {m }}$ (Covidien, New Haven, CT, USA)). Next, a jejunal limb was pulled up in an antecolic position after which an antegastric end-to-side gastrojejunostomy was created by linear stapling and sutures using Polysorb ${ }^{\text {TM }}$ sutures before 2010 and V-Loc ${ }^{\text {TM }}$ (Covidien, Mansfield, MA, USA) sutures since then.

Until 2010, the alimentary limb measured an estimated $100-150 \mathrm{~cm}$. Since 2010, the alimentary $\operatorname{limb}$ measured an estimated $150-180 \mathrm{~cm}$. Often, the procedure was ended by closure of the mesenteric defect.

\section{Statistical analysis}

Data were collected and analyzed retrospectively. Data management and analysis was performed using SPSS version 22 for Windows (IBM Corporation, Armonk, NY, USA). Quantitative data are denoted as mean +/- standard deviation (SD). Complications and rate of readmissions are presented as a percentage.

Either student $t$-test, Mann Whitney U-test or Chi-square-test was used to determine any statistical significance between the observed differences among groups depending on distribution. Differences were considered significant in case of a $p$ value less than 0.05 . Odds Ratio (OR) and $95 \%$ confidence intervals (Cl) were provided when applicable and considered significant when OR $(95 \%$ Cl) $\neq 1$.

Summative figures and tables were used when applicable.

No ethical approval was required for this study. Permission was granted by the Institutional Review Board for this retrospective study.

All procedures performed in study involving human participants were in accordance with the ethical standards of the institutional research committee and with the 1964 Helsinki declaration and its later amendments or comparable ethical standards. For this type of study, formal consent was not required.

Informed consent was obtained from all individual participants included in the study.

\section{RESULTS}

A total of 178 patients ( $79.8 \%$ female) underwent CRYGB after either primary AGB or primary SG between January 2007 and December 2012. Patient characteristics are presented in Table 6.1, divided between the group of patients with AGB as primary procedure $(n=110 ; 61.8 \%)$ and the group with SG as primary procedure $(n=68 ; 38.2 \%)$. 
Average operating time was $86.3 \pm 37.3$ minutes (AGB 92.7 \pm 39.3 vs. SG $75.9 \pm 31.3 ; p=<0.001$ ). Mean hospital stay was $3.8 \pm 5.9$ days after surgery (AGB $4.3 \pm 7.0$ vs. SG $3.0 \pm 3.5 ; p=0.143$ ). A total of six procedures (3.4\%) were converted from laparoscopic to open procedure (AGB $n=5$ vs. $S G n=1$; $p=0.409$ ). One patient underwent conversion due to persistent bleeding of the omentum, one procedure was converted due to splenic hemorrhage and four procedures were converted because of an insufficient view due to extensive adhesions. 30-day mortality rate was zero. No patients died during the 2-year follow-up of the study.

Table 6.1 Patient characteristics $(n=178)$.

\begin{tabular}{lccc}
\hline & $\begin{array}{c}\text { AGB } \\
\text { n=110 (61.8\%) } \\
\text { Mean } \pm \text { SD }\end{array}$ & $\begin{array}{c}\text { SG } \\
\mathbf{n = 6 8} \text { (38.8\%) } \\
\text { Mean } \pm \text { SD }\end{array}$ & p-value \\
\hline Age (years) & $44.1 \pm 8.8$ & $44.7 \pm 11.1$ & 0.735 \\
Male : Female $(\mathrm{n})$ & $91: 19$ & $51: 17$ & 0.212 \\
Body Mass Index (BMI) $\left(\mathrm{kg} / \mathrm{m}^{2}\right)$ & $39.4 \pm 6.2$ & $36.1 \pm 7.7$ & $\mathbf{0 . 0 0 2}$ \\
Highest registered BMI $\left(\mathrm{kg} / \mathrm{m}^{2}\right)$ & $45.9 \pm 6.3$ & $50.6 \pm 10.5$ & $\mathbf{0 . 0 0 3}$ \\
Starting BMI (before primary surgery) $\left(\mathrm{kg} / \mathrm{m}^{2}\right)$ & $44.9 \pm 6.0$ & $49.3 \pm 10.7$ & $\mathbf{0 . 0 1 0}$ \\
Pre-operative comorbidities $(\mathrm{n}, \%)$ & & & 0.380 \\
Diabetes Mellitus type 2 & 17 & 14 & $\mathbf{0 . 0 3 4}$ \\
Hypertension & 23 & 10 & 0.171 \\
Dyslipidemia & 9 & 8 & 0.129 \\
Sleep apnea & 6 & 7 & 0.481 \\
Osteo-articular disease & 8 & & \\
ASA classification (n, \%) & & 3 & 0.595 \\
I & 3 & 60 & 0.525 \\
II & 102 & 5 & $17: 51$ \\
III & 5 & & \\
Conventional care : fast track care & $23: 87$ & & \\
\hline
\end{tabular}

AGB: adjustable gastric band; SG: sleeve gastrectomy; SD: standard deviation.

\section{(Technical) reasons for conversion}

Table 6.2 describes the reasons which led to the conversion of the primary bariatric procedure. Main reasons were either weight regain $(33.7 \%)$ or dysphagia (30.3\%).

Four patients had other complaints after AGB. One patient had persisting pain complaints, one patient had a leaking band with no weight regain until that moment, and one patient had many complaints after AGB which eventually necessitated removal of the band and one patient necessitated CRYGB for a better regulation of the known type 2 diabetes mellitus.

Patients often underwent additional imaging (often swallow test or gastroscopy) to further analyze the complaints of the patient. Additional analysis showed band slippage in 19 (17.3\%) patients after AGB, late infection 
of the AGB in three patients (2.8\%), pouch dilatation above the AGB in two patients $(1.8 \%)$ and a leaking band or band erosion in two patients each (1.8\%). In 82 patients (74.5\%) after AGB, either no evident technical cause for failure was found or no additional analysis was performed.

After SG, additional analysis showed dilatation of the sleeve in three patients (4.4\%), kinking/torsion of the sleeve (causing persistent dysphagia or reflux complaints) in four patients (5.9\%) and stenosis of the sleeve in nine patients (13.2\%). In 52 patients (76.5\%), no evident technical cause was found or patients did not undergo additional analysis of complaints.

Table 6.2 Reasons for conversion $(n=178)$.

\begin{tabular}{|c|c|c|c|}
\hline & $\begin{array}{c}\text { AGB } \\
n=110(61.8 \%) \\
n\end{array}$ & $\begin{array}{c}\text { SG } \\
n=68(38.8 \%) \\
n\end{array}$ & $p$-value \\
\hline Weight regain & $56(50.9 \%)$ & $4(5.9 \%)$ & $<0.001$ \\
\hline Insufficient weight loss & $17(15.5 \%)$ & $11(16.2 \%)$ & 0.898 \\
\hline Dysphagia & $27(24.5 \%)$ & $27(39.7 \%)$ & 0.033 \\
\hline Reflux/GERD & $6(5.5 \%)$ & $11(16.2 \%)$ & 0.018 \\
\hline Planned $2^{\text {nd }}$ bariatric procedure & 0 & $15(22.0 \%)$ & $<0.001$ \\
\hline Other & $4(3.6 \%)$ & 0 & 0.112 \\
\hline
\end{tabular}

AGB: adjustable gastric band; SG: sleeve gastrectomy; GERD: Gastro esophageal reflux disease.

\section{Intra- and postoperative complications $<\mathbf{3 0}$ days}

Intra-operative complications occurred in a total of six patients (3.4\%): five after failed AGB (4.5\%) versus one after failed SG (1.5\%), $p=0.409$.

Early postoperative surgical and/or infectious complications occurred in a total of 21 patients (11.8\%), 15 after AGB and 6 after SG. These complications are displayed in Table 6.3.

\section{Weight loss and comorbidity evolution}

Weight loss was evaluated after, six months, one year and two years. Weight loss parameters (\%EWL, \%TBWL and CBMI) are shown in Table 6.4. The percentages of the obesity-related comorbidities which either were improved or resolved after two years were $91.3 \%, 87.5 \%, 86.7 \%, 72.7 \%$ and $69.2 \%$ for respectively diabetes mellitus type 2 , hypertension, dyslipidemia, sleep apnea and osteo-articular disease in this study population. 
Table 6.3 Postoperative outcome $(n=178)$.

\begin{tabular}{lccc}
\hline & $\begin{array}{c}\text { AGB } \\
\mathbf{n = 1 1 0} \\
\text { Mean } \mathbf{1} \text { SD }\end{array}$ & $\begin{array}{c}\text { SG } \\
\mathbf{n}=\mathbf{6 8} \\
\text { Mean } \pm \text { SD }\end{array}$ & p-value \\
\hline Patients with surgical complications (n, \%) & $13(11.8 \%)$ & $6(8.8 \%)$ & 0.530 \\
Leakage gastrojejunostomy (n, \%) & $6(5.5 \%)$ & $3(4.4 \%)$ & 1.000 \\
Leakage enteroenterostomy (n, \%) & 0 & 0 & $n / a$ \\
Bleeding (n, \%) & $4(3.6 \%)$ & $3(4.4 \%)$ & 1.000 \\
Gastro-intestinal perforation (n, \%) & $3(2.7 \%)$ & 0 & 0.288 \\
Patients with infectious complications & $11(10 \%)$ & $2(2.9 \%)$ & 0.135 \\
Intra-abdominal abscess (n, \%) & $8(7.3 \%)$ & $2(2.9 \%)$ & 0.322 \\
Pneumonia (n,\%) & $3(2.7 \%)$ & 0 & 0.288 \\
Urinary tract infection & 0 & 0 & $\mathrm{n} / \mathrm{a}$ \\
Wound infection & $1(0.9 \%)$ & 0 & 1.000 \\
Clavien-Dindo 1 (n, \%) & 0 & 0 & $\mathrm{n} / \mathrm{a}$ \\
Clavien-Dindo 2 (n, \%) & $4(3.6 \%)$ & 0 & 0.299 \\
Clavien-Dindo 3a/3b (n, \%) & $10(9.1 \%)$ & $6(8.8 \%)$ & 0.952 \\
Clavien-Dindo 4a/4b (n, \%) & $1(0.9 \%)$ & 0 & 1.000 \\
Clavien-Dindo 5 (n, \%) & 0 & 0 & $\mathrm{n} / \mathrm{a}$ \\
Serious Adverse Events (Clavien-Dindo $\geq 3)(\mathrm{n}, \%)$ & $11(10 \%)$ & $6(8.8 \%)$ & 0.795 \\
\hline
\end{tabular}

Missing Data: $n=0$. AGB: adjustable gastric band; SG: sleeve gastrectomy; SD: standard deviation.

Table 6.4 Weight loss after CRYGB $(n=178)$.

\begin{tabular}{|c|c|c|c|c|}
\hline & $\begin{array}{c}\text { AGB } \\
n=110 \\
\text { Mean } \pm \text { SD }\end{array}$ & $\begin{array}{c}\text { SG } \\
n=68 \\
\text { Mean } \pm \text { SD }\end{array}$ & $p$-value & $\begin{array}{c}\text { Missing } \\
\text { data } \\
\text { n (\%) }\end{array}$ \\
\hline \multicolumn{5}{|l|}{ Weight loss after 6 months } \\
\hline Change in $\mathrm{BMI}\left(\mathrm{kg} / \mathrm{m}^{2}\right)$ & $13.3 \pm 5.9$ & $16.5 \pm 6.9$ & 0.001 & $44(24.7 \%)$ \\
\hline \% Total Body Weight Loss (\%TBWL) & $28.3 \pm 9.6$ & $33.4 \pm 10.3$ & 0.004 & $44(24.7 \%)$ \\
\hline \% Excess Weight Loss (\%EWL) & $63.7 \pm 19.1$ & $74.3 \pm 27.3$ & 0.014 & $44(24.7 \%)$ \\
\hline \multicolumn{5}{|l|}{ Weight loss after 1 year } \\
\hline Change in $\mathrm{BMI}\left(\mathrm{kg} / \mathrm{m}^{2}\right)$ & $14.4 \pm 6.0$ & $17.1 \pm 7.5$ & 0.021 & $42(23.6 \%)$ \\
\hline \% Total Body Weight Loss (\%TBWL) & $31.1 \pm 9.6$ & $33.8 \pm 11.9$ & 0.144 & $42(23.6 \%)$ \\
\hline \% Excess Weight Loss (\%EWL) & $71.7 \pm 19.0$ & $73.2 \pm 29.2$ & 0.735 & 42 (23.6\%) \\
\hline \multicolumn{5}{|l|}{ Weight loss after 2 years } \\
\hline Change in $\mathrm{BMI}\left(\mathrm{kg} / \mathrm{m}^{2}\right)$ & $14.5 \pm 6.5$ & $15.7 \pm 7.0$ & 0.373 & $62(34.8 \%)$ \\
\hline \% Total Body Weight Loss (\%TBWL) & $31.6 \pm 11.0$ & $31.6 \pm 12.0$ & 0.998 & 62 (34.8\%) \\
\hline \% Excess Weight Loss (\%EWL) & $73.1 \pm 23.1$ & $68.3 \pm 28.6$ & 0.324 & 62 (34.8\%) \\
\hline
\end{tabular}

CRYGB: conversion to Roux-en-Y gastric bypass; AGB: adjustable gastric band; SG: sleeve gastrectomy; SD: standard deviation.

\section{Subgroup analysis}

Subgroup analysis was performed to assess differences in complications and weight loss after two years between the two groups with the primary aim to obtain additional weight loss (weight regain, insufficient weight loss or planned second bariatric procedure). A total of 103 (57.9\%) were included (AGB: $n=73$ 
vs. SG: $\mathrm{n}=30$ ). No differences were found at baseline except starting BMI (AGB $45.0 \pm 6.1 \mathrm{~kg} / \mathrm{m}^{2}$ vs. SG $\left.55.1 \pm 11.3 \mathrm{~kg} / \mathrm{m}^{2} ; p<0.001\right)$. No differences were found in terms of both postoperative complications (AGB $15.1 \%$ vs. SG 6.7\%; $p=0.337$ ). Out of the 103 patients included in this subgroup, 66 (64.1\%) attended the 2-year follow-up. Weight loss was not different in terms of cBMI (AGB $14.9 \pm$ $7.2 \mathrm{~kg} / \mathrm{m}^{2}$ vs. SG $15.0 \pm 8.1 \mathrm{~kg} / \mathrm{m}^{2} ; p=0.951$ ) or \%TBWL (AGB $31.7 \pm 11.7 \%$ vs. SG $27.0 \pm 13.1 \% ; p=0.173$ ), however it did differ in terms of \%EWL (AGB $71.1 \pm 21.6 \%$ vs. SG $50.8 \pm 23.0 \% ; p=0.002$ ).

Furthermore, patients undergoing conversion for relief of complaints were analyzed (AGB: $n=37$ vs. SG: $n=38$ ). Differences at baseline were a higher prevalence of type 2 diabetes mellitus $(p=0.007)$, hypertension $(p=0.017)$ and dyslipidemia $(p=0.047)$ in SG patients. Furthermore, the pre-operative BMI was different (AGB $36.7 \pm 6.5 \mathrm{~kg} / \mathrm{m}^{2}$ vs. SG $31.8 \pm 5.7 \mathrm{~kg} / \mathrm{m}^{2} ; p=0.001$ ).

Results showed no difference in postoperative complications (AGB $10.8 \%$ vs. SG $10.5 \% ; p=1.000$ ). Out of the 75 patients included, 50 (66.7\%) attended the 2-year follow-up. No differences were found in weight loss after two years in terms of cBMI (AGB $13.8 \pm 4.9 \mathrm{~kg} / \mathrm{m}^{2}$ vs. SG $16.2 \pm 6.3 \mathrm{~kg} / \mathrm{m}^{2} ; p=0.951$ ) and \%TBWL (AGB $31.4 \pm 9.9 \%$ vs. SG $34.8 \pm 10.3 \% ; p=0.173$ ), whereas \%EWL differed significantly. (AGB $76.9 \pm 25.8 \%$ vs. SG $80.7 \pm 25.9 \% ; p=0.002$ ). Pre-operative complaints improved in $100 \%$ in the failed AGB group and in $71 \%$ in the failed SG group $(p<0.001)$.

\section{DISCUSSION}

This is the first study to compare the short-term outcome of CRYGB after failed $A G B$ and SG. Main differences between the two groups were the starting BMI and the reasons for conversion. In terms of postoperative complications, weight loss and comorbidity-improvement, results were similar between conversion to AGB and conversion to SG.

At baseline, both current $\mathrm{BMI}$, as well as starting $\mathrm{BMI}$ before primary procedure differed significantly. Taking a closer look at the current study population, the results suggest a better initial weight loss effect after SG compared to AGB, as SG patients started off at a higher BMI and ended up at a lower BMl after primary surgery. Caution is needed when discussing the effect of the primary procedures, as the time between the primary and secondary procedure is an important factor, but is unknown in many patients of the current study population. Many of these patients were referred from different hospitals which compromised some of these data. 
Operative time was longer in the AGB-group, which may be caused by the longer time needed in patients in which band removal and CRYGB were performed sequentially in the same procedure. Another explanation may very well be the fact that CRYGB after failed AGB is more challenging than CRYGB after failed SG.

No differences were found in both total as well as individual postoperative complications between the two groups, making the hypothesis that CRYGB after failed SG may lead to less postoperative complications unlikely. On the other hand, these complication rates do emphasize the complexity of secondary bariatric procedures, as they are higher compared to reported complication rates after primary RYGB. ${ }^{19,20}$ Nevertheless, they are comparable with previous reports on secondary procedures..$^{8-10,21}$ The majority of the AGB patients was operated in two stages; first the removal of the band and after approximately three months the RYGB. This did not decrease the amount of postoperative complications as similar results were found after one-step conversion. ${ }^{22,23}$ In this study, when considering the BMI before primary surgery as reference point, average weight loss two years after conversion was similar between the two groups. Weight loss differed six months after surgery, which is most likely still explained by the large difference in mean BMI before secondary surgery.

The most essential differences are found in the reason for conversion and its associated subgroup analyses, since the majority of the failed AGB-patients underwent conversion for additional weight loss, whereas the distribution between the aim for additional weight loss and complaint relief was more equal in SG patients. Conversions aiming to establish additional weight loss showed a higher percentage of complications after conversion of a failed AGB, although not significant. In terms of weight loss, CBMI and \%TBWL were similar. The \%EWL was significantly higher in failed AGB patients, however recent literature suggested that \%EWL may not be the best way to describe weight loss.(24) On the other hand, the large absolute difference in \%EWL, while taking the starting $\mathrm{BMI}$ of both groups into account, may suggest that CRYGB as additional weight loss procedure after SG might not be the best option for achieving additional weight loss after failed SG. Looking at newly available literature, for example duodenal switch or single anastomosis duodeno-ileal bypass appear to have the upper hand over RYGB in terms of additional weight loss, however serious postoperative deficiencies and other (unknown) long-term complications have to be taken into account when considering these procedures. ${ }^{13,14}$ The results after failed AGB are good when comparing them to several other studies, confirming that RYGB is a good re-do option after failed AGB for additional weight loss. ${ }^{25}$ 
When analyzing the patients undergoing conversion for relief of complaints, SG-patients showed a significant lower pre-operative BMI. The therapeutic mechanism however is very different between the two groups. Complaints of patients in the AGB group are usually relieved after removal of the band since the anatomic state is returned to normal, whereas in SG-patients, the CRYGB is the therapeutic procedure, since return to normal anatomy is impossible. After band removal, weight regain seems inevitable for which CRYGB appears to be a good option to prevent that when looking at the current results, as it even achieves additional weight loss. Furthermore, CRYGB is a valuable option for SG-patients with complaints as it achieves relief of complaints in many patients and establishes good short-term weight stabilization in the current study. These findings do suggest that the effect of the RYGB as a secondary procedure is dependent on the combination of the reason for which a conversion is performed and the primary bariatric procedure. A thorough assessment of these patients is advisable to make a clear distinction between these different groups. In terms of weight loss, RYGB seems feasible after failed AGB. In terms of relief of complaints, RYGB seems feasible after either AGB or SG.

However, in terms of weight loss, RYGB may not be the best option after SG, especially since other procedures such as SADI seem preferable in terms of (additional) weight loss after SG. ${ }^{14,26}$ Further research is needed to substantiate these assumptions.

The current study design is subject to limitations, since the retrospective design makes the results subjective to bias and sometimes incomplete when looking at the follow-up. Furthermore, the follow-up, especially the 2-year follow-up, shows a high number of missing which compromises the impact and the credibility of the current results. Despite these limitations, it can be concluded that RYGB as secondary procedure after failed AGB or failed SG shows comparable short-term results in terms of postoperative complications and weight loss. Nonetheless, these results are susceptible to the reason that necessitates conversion. 


\section{REFERENCES}

1. O'Brien PE, MacDonald L, Anderson M, Brennan L, Brown WA. Long-term outcomes after bariatric surgery: fifteen-year follow-up of adjustable gastric banding and a systematic review of the bariatric surgical literature. Ann Surg. 2013;257(1):87-94.

2. DeMaria EJ, Sugerman HJ, Meador JG, Doty JM, Kellum JM, Wolfe L, et al. High failure rate after laparoscopic adjustable silicone gastric banding for treatment of morbid obesity. Ann Surg. 2001;233(6):809-18.

3. Aarts EO, Dogan K, Koehestanie P, Aufenacker TJ, Janssen IM, Berends FJ. Long-term results after laparoscopic adjustable gastric banding: a mean fourteen year follow-up study. Surg Obes Relat Dis. 2014;10(4):633-40.

4. van Rutte PW, Smulders JF, de Zoete JP, Nienhuijs SW. Outcome of sleeve gastrectomy as a primary bariatric procedure. Br J Surg. 2014;101(6):661-8.

5. Vidal P, Ramon JM, Goday A, Benaiges D, Trillo L, Parri A, et al. Laparoscopic gastric bypass versus laparoscopic sleeve gastrectomy as a definitive surgical procedure for morbid obesity. Mid-term results. Obes Surg. 2013;23(3):292-9.

6. Wang MC, Guo XH, Zhang YW, Zhang YL, Zhang HH, Zhang YC. Laparoscopic Roux-en-Y Gastric Bypass versus Sleeve Gastrectomy for Obese Patients with Type 2 Diabetes: A Metaanalysis of Randomized Controlled Trials. Am Surg. 2015;81(2):164-9.

7. Colquitt JL, Pickett K, Loveman E, Frampton GK. Surgery for weight loss in adults. The Cochrane database of systematic reviews. 2014;8:CD003641.

8. Gautier T, Sarcher T, Contival N, Le Roux Y, Alves A. Indications and mid-term results of conversion from sleeve gastrectomy to Roux-en-Y gastric bypass. Obes Surg. 2013;23(2): 212-5.

9. van Rutte PW, Smulders JF, de Zoete JP, Nienhuijs SW. Indications and short-term outcomes of revisional surgery after failed or complicated sleeve gastrectomy. Obes Surg. 2012;22(12): 1903-8.

10. Marin-Perez P, Betancourt A, Lamota M, Lo Menzo E, Szomstein S, Rosenthal R. Outcomes after laparoscopic conversion of failed adjustable gastric banding to sleeve gastrectomy or Roux-en-Y gastric bypass. Br J Surg. 2014;101(3):254-60.

11. Perathoner A, Zitt M, Lanthaler M, Pratschke J, Biebl M, Mittermair R. Long-term follow-up evaluation of revisional gastric bypass after failed adjustable gastric banding. Surg Endosc. 2013;27(11):4305-12.

12. Jennings NA, Boyle M, Mahawar K, Balupuri S, Small PK. Revisional laparoscopic Roux-en-Y gastric bypass following failed laparoscopic adjustable gastric banding. Obes Surg. 2013; 23(7):947-52.

13. Homan J, Betzel B, Aarts EO, van Laarhoven KJ, Janssen IM, Berends FJ. Secondary surgery after sleeve gastrectomy: Roux-en-Y gastric bypass or biliopancreatic diversion with duodenal switch. Surg Obes Relat Dis. 2015;11(4):771-7.

14. Sanchez-Pernaute A, Rubio MA, Conde M, Arrue E, Perez-Aguirre E, Torres A. Singleanastomosis duodenoileal bypass as a second step after sleeve gastrectomy. Surg Obes Relat Dis. 2015;11(2):351-5.

15. Mognol P, Chosidow D, Marmuse JP. Laparoscopic conversion of laparoscopic gastric banding to Roux-en-Y gastric bypass: a review of 70 patients. Obes Surg. 2004;14(10):1349-53.

16. van Wageningen B, Berends FJ, Van Ramshorst B, Janssen IF. Revision of failed laparoscopic adjustable gastric banding to Roux-en-Y gastric bypass. Obes Surg. 2006;16(2):137-41.

17. Dindo D, Demartines N, Clavien PA. Classification of surgical complications: a new proposal with evaluation in a cohort of 6336 patients and results of a survey. Ann Surg. 2004;240(2): 205-13.

18. Deitel M, Greenstein RJ. Recommendations for reporting weight loss. Obes Surg. 2003; 13(2):159-60. 
19. Shikora SA, Kim JJ, Tarnoff ME, Raskin E, Shore R. Laparoscopic Roux-en-Y gastric bypass: results and learning curve of a high-volume academic program. Arch Surg. 2005;140(4):362-7.

20. Suter M, Donadini A, Romy S, Demartines N, Giusti V. Laparoscopic Roux-en-Y gastric bypass: significant long-term weight loss, improvement of obesity-related comorbidities and quality of life. Ann Surg. 2011;254(2):267-73.

21. Berende CA, de Zoete JP, Smulders JF, Nienhuijs SW. Laparoscopic sleeve gastrectomy feasible for bariatric revision surgery. Obes Surg. 2012;22(2):330-4.

22. Aarts $E$, Koehestanie $P$, Dogan $K$, Berends $F$, Janssen I. Revisional surgery after failed gastric banding: results of one-stage conversion to RYGB in 195 patients. Surg Obes Relat Dis. 2014; 10(6):1077-83.

23. Stroh C, Weiner R, Wolff S, Lerche C, Knoll C, Keller T, et al. One versus two-step Roux-en-Y gastric bypass after gastric banding-data analysis of the German Bariatric Surgery Registry. Obes Surg. 2015;25(5):755-62.

24. van de Laar A, de Caluwe L, Dillemans B. Relative outcome measures for bariatric surgery. Evidence against excess weight loss and excess body mass index loss from a series of laparoscopic Roux-en-Y gastric bypass patients. Obes Surg. 2011;21(6):763-7.

25. Coblijn UK, Verveld CJ, van Wagensveld BA, Lagarde SM. Laparoscopic Roux-en-Y gastric bypass or laparoscopic sleeve gastrectomy as revisional procedure after adjustable gastric band--a systematic review. Obes Surg. 2013;23(11):1899-914.

26. Carmeli I, Golomb I, Sadot E, Kashtan H, Keidar A. Laparoscopic conversion of sleeve gastrectomy to a biliopancreatic diversion with duodenal switch or a Roux-en-Y gastric bypass due to weight loss failure: our algorithm. Surg Obes Relat Dis. 2015;11(1):79-85. 



\section{CHAPTER 7}

\section{Matched short-term results of SADI versus GBP after sleeve gastrectomy}

C.M.M. Ceha

M.R. van Wezenbeek

D.P.A. Versteegden

J.F. Smulders

S.W. Nienhuijs

Obes Surg. 2018;28(12):3809-14

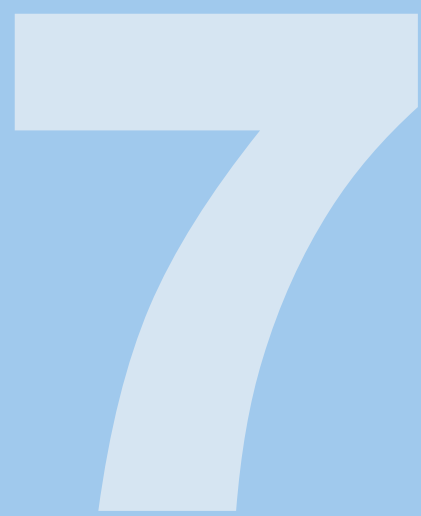




\section{ABSTRACT}

\section{Background}

The Gastric Bypass (GBP) is the most popular revisional technique after Sleeve Gastrectomy (SG). However, the results after revision are not always satisfactory in terms of additional weight loss and complications. The Single Anastomosis Duodenolleal bypass (SADI) could be a valuable alternative.

\section{Objective}

This study is a retrospective matched-control study reviewing short term results in terms of weight loss and comorbidities reduction of both SADI and GBP as a revisional procedure after primary sleeve gastrectomy. Complications and vitamin deficiencies will be evaluated as well.

\section{Methods}

Patients with a SADI procedure after a primary sleeve gastrectomy (SG) with a minimum follow-up of one year, were included. Their results were retrospectively reviewed and matched with a cohort of GBP patient on age, BMI and gender. Comparison was on comorbidities, weight loss, complications and blood markers.

\section{Results}

A total of 64 patients were included, 32 SADI procedures and 32 matched gastric bypasses with no significant differences on baseline. No differences were found in terms of (additional) total weight loss. The operating time of the SADI was significantly longer $(p=0.007)$. No clinically relevant differences were found concerning comorbidities or blood markers. In the SADI group, more defecation problems were reported and fewer vitamins deficiencies were encountered

\section{Conclusion}

Based on a small group and short-term results, the SADI could be regarded as a safe alternative to a GBP following SG with a similar amount of additional weight loss and fewer post-operative early complications. Longer follow-up and larger studies are needed to determine its full potential. 


\section{INTRODUCTION}

Obesity is still a globally expanding problem and is even called an epidemic. In the Netherlands alone, almost $14 \%$ of all the citizens is obese.(1) The risk at disease increases as the Body Mass Index (BMI) or waist circumference increases. ${ }^{1,2}$ Bariatric surgery is currently the most effective therapy for obesity with good long-term results. A multitude of studies showed significant weight loss and substantial improvement of comorbidities after bariatric surgery. ${ }^{3}$ Weight loss is achieved by multiple standard procedures such as a laparoscopic sleeve gastrectomy (SG), a Roux-en-Y gastric bypass (GBP) or a duodenal switch (DS).

Patients suffering from a more severe form of morbid obesity (BMI>60), benefit the most from bariatric surgery. Those patients may even qualify for a two-step procedure. This often includes a SG as primary bariatric procedure and conversion to a GBP as revisional procedure. ${ }^{4}$ However, earlier research showed that the combination of SG and GBP surgery often shows insufficient results compared to the combination of SG and DS. ${ }^{5}$ The drawback of the DS may be a technically more challenging operation. For this reason, the Single Anastomosis Duodenolleal Bypass (SADI) was developed by Torres et al. ${ }^{6}$ It can be regarded as a technically less demanding procedure with presumably a similar amount of weight loss. ${ }^{6,7}$ The first clinical results of the SADI as a primary procedure, with a follow-up period of three years showed an excess weight loss of almost $100 \%$ after three years. Nonetheless, a comparison with other procedures is missing. ${ }^{6}$ Therefore, this study will present the clinical results concerning the differences between the results of the SADI and what is currently considered the gold standard; GBP following a SG. Results of both surgery types will be defined by means of comorbidities, weight loss, complications and blood markers.

\section{METHODS}

This is a retrospective matched-cohort study, for which medical charts were reviewed to retrieve the necessary data. Informed consent was obtained from all individual participants. A total of six follow-up moments were included in the study: 1. Before sleeve gastrectomy (baseline characteristics), 2. One year after sleeve gastrectomy, 3. Before revisional surgery, 4. Three months after revision, 5. Six months after revision, 6 . One year after revision. During these follow-up moments, a number of parameters were measured and reviewed. The primary outcome for this study is weight loss after revisional surgery, expressed in percentage of total weight loss (\%TWL). \%TWL is calculated as (initial weight - 
current weight) / (initial weight) * $100 \%$. $\%$ TWL was chosen to report weight loss since it appears to be less influenced by preoperative BMI in comparison to the percentage of excess weight loss (\%EWL). ${ }^{8,9}$

Secondary parameters are \%TWL after revision, the evolution of any known obesity-related comorbidities, various blood markers, operating time and postoperative complications.

To calculate the weight loss effect of the conversion alone, \%TWL after conversion is calculated: (weight at revision - weight at follow-up) / (weight at revision) * $100 \%$.

All known obesity-related comorbidities (diabetes mellitus type 2, hypertension, dyslipidemia, obstructive sleep apnea syndrome (OSAS), joint complaints and gastro-esophageal reflux disease (GERD)) are scored at baseline. The evolution of these comorbidities is categorized as either stable, improved, resolved, worse, de novo or not applicable.

The presence and potential improvement of diabetes mellitus type 2 will be assessed by the amount of antidiabetic medication used and the HbA1c-level. When the HbA1c-level is lower than $42 \mathrm{mmol} / \mathrm{mol}$ and no antidiabetic medication is necessary, the diabetes is considered resolved. An improvement is considered when a reduction in use of medication is achieved or a lower $\mathrm{HbA1c}$-level is measured. A decline is considered when the opposite occurs.

The presence and potential improvement of hypertension will be assessed by measuring the blood pressure and evaluating the current use of medication. Hypertension is considered resolved when measuring a normal blood pressure (120-140/80-89 $\mathrm{mmHg}$ ) without the use of medication. Improvement is considered with a $50 \%$ reduction in medication dose, stopping one or more medicines or achieving a normal tension with the current medication. A decline is considered when the opposite occurs.

The presence and potential improvement of hypercholesterolemia will be assessed by LDH/HDL ratio and potential changes in the use of medication.

The presence and potential improvement obstructive sleep apnea is assessed by the use of a continuous positive airway pressure (CPAP) device and the evolution of complaints (subjective improvement or decline).

Joint complaints are only assessed by the subjective amount of complaints of the patient and the use of pain medication for joint complaints.

Blood markers include cholesterol ( $\mathrm{mmol} / \mathrm{L}), \mathrm{LDL}(\mathrm{mmol} / \mathrm{L}), \mathrm{HDL}(\mathrm{mmol} / \mathrm{L})$, $\mathrm{HbA1c}(\mathrm{mmol} / \mathrm{mol}), \mathrm{C}$-peptide $(\mathrm{ng} / \mathrm{mL})$, insulin (pmol/L), creatinine $(\mu \mathrm{mol} / \mathrm{L})$, iron $(\mu \mathrm{mol} / \mathrm{L})$, ferritin $(\mathrm{g} / \mathrm{L})$, vitamin $\mathrm{B} 12(\mathrm{pmol} / \mathrm{L})$ and vitamin $\mathrm{D}(\mathrm{nmol} / \mathrm{L})$.

The postoperative complications include postoperative bleeding, internal herniation, anastomotic leakage, vitamin B12 and vitamin D deficiencies, defecation complaints (either obstipation; no defecation for at least 3 days, diarrhea; Bristol stool scale 7 or fatty stools; bulky stools, difficult to flush, pale 
and oily appearance, foul-smelling), emergency care visits and emergency surgery. Vitamin B12 and D deficiencies are determined by use of reference values.

\section{Study population}

All patients who underwent conversion from sleeve gastrectomy to SADI between January 2016 and June 2017 with an available minimum amount of follow-up of 6 months will be considered for inclusion in the current study. Patients who had surgery before 2013 were not included, due to changes in pre- and postoperative protocol. These patients will be used to match the included SADI-patients on age, sex and BMI. The BMI had a range of maximum $10 \mathrm{~kg} / \mathrm{m}^{2}$ per individual match and the maximum age difference was 10 years. Before surgery, patients were screened for physical and mental condition using interviews and tests with an obesity nurse, psychologist, dietician, and physiotherapist. Based on the opinions of the mentioned professionals, a multidisciplinary consultation concluded whether the patient was appropriate to undergo revisional surgery. Measurements during this screening were used as baseline characteristics.

\section{Surgical procedures}

The pouch of the gastric bypass procedure was created by dissecting the prior sleeve using the Endo-GIA ${ }^{\text {m }}$ stapler (Medtronic, New Haven, CT, USA). The antecolic and antegastric gastrojejunal and jejuno-jejunostomy anastomosis were created, using an EndoGIA ${ }^{\mathrm{m}}$ and $\mathrm{V}$-loc ${ }^{\mathrm{jm}}$ stitching. The alimentary limb measured approximately $150-180 \mathrm{~cm}$ and the biliopancreatic limb is $60-80 \mathrm{~cm}$. Both internal openings were closed. A leak test was performed to test the gastrojejunostomy. The SADI procedure encompassed a Maryland dissection along the sleeve up to the level of the gastroduodenal artery, under the pylorus, preserving the vascularization at the lesser curvature. The duodenum was divided with an Endo-GIA ${ }^{\mathrm{m}}$. The ileocecal junction was identified and measured proximally up to around $250 \mathrm{~cm}$. The loop was ascended antecolic and a side-to-side duodenoileal anastomosis was created with $2 \mathrm{~V}$-loc ${ }^{\mathrm{m}}{ }^{\mathrm{m}}$ stiches. A leak test was performed.

All subjects followed the same postoperative protocol including a liquid diet for two weeks, the use of low-molecular weight heparins (Fragmin ${ }^{\text {mM }}{ }^{\text {) }}$ ) for four weeks to prevent thrombo-embolic events and the use of pantoprazole for three months, to reduce complaints of gastric acid. Finally, life-long vitamin supplementation is advised. 


\section{Statistical analysis}

The results were presented as mean \pm standard deviation (SD). A $p$-value $<0.05$ was considered to be statistically significant. Data management and analysis was performed using SPSS version 23 for Windows (IBM Corporation, Armonk, NY, USA).

One-way ANOVA's were performed to test differences between groups concerning the percentage TWL, BMl, blood markers, operating time, highest and lowest weight. When the Levene's test for homogeneity of variance proved that the assumption of equal variances was not met, the Brown-Forsythe for testing the equality of means was performed. Concerning dichotomous variables, chi-square tests or, when indicated, Fischer's exact tests were performed per variable.

All procedures performed in studies involving human participants were in accordance with the ethical standards of the institutional and/or national research committee and with the 1964 Helsinki declaration and its later amendments or comparable ethical standards.

\section{RESULTS}

In total, 42 patients underwent a conversion to SADI between January 2016 and June 2017. Nine patients were excluded due to an insufficient follow-up period. One patient was excluded since the procedure considered a conversion from GBP to SADI. A total of 86 patients were selected from all patients who underwent a revisional GBP between 2013 and 2016. Thirty-two out of them were matched to the SADI-patients.

Demographics of the study population at baseline are shown in Table 7.1. No cases of intraoperative complications were reported in this study population. The mean operating time of the SADI was significantly higher than the operating time of the GBP (66.6 min vs. 56.9 min; $p=0.006$ ).

The indication for conversion to SADI was a planned secondary procedure to achieve additional weight loss after primary SG in patients with a high initial $\mathrm{BMI}$. All conversions from SG to GBP were due to either insufficient weight loss or weight regain after primary SG. 
Table 7.1 Demographics.

\begin{tabular}{lccc}
\hline & SADI & Gastric bypass & p-value \\
\hline Participants $(\mathrm{n})$ & 32 & 32 & $\mathrm{n} / \mathrm{a}$ \\
Male $(\mathrm{n})$ & $6(18.8 \%)$ & $6(18.8 \%)$ & $\mathrm{n} / \mathrm{a}$ \\
Female $(\mathrm{n})$ & $26(81.3 \%)$ & $26(81.3 \%)$ & $\mathrm{n} / \mathrm{a}$ \\
Age $($ mean, range) & $46.9(21-66)$ & $48.2(29-63)$ & 0.597 \\
Mean BMI \pm SD $\left(\mathrm{kg} / \mathrm{m}^{2}\right)$ & $57.5( \pm 7.6)$ & $53.7( \pm 7.0)$ & $\mathbf{0 . 0 3 0}$ \\
\hline
\end{tabular}

BMI: body mass index; SD: Standard deviation.

\section{Complications}

Postoperative complications and long-term complications are displayed in Table 7.2.

The largest difference was found in defecation problems, which was a $21.9 \%$ difference between the two groups $(p=0.037)$. Vitamin B12 and D deficiencies were defined for multiple follow-up moments (Table 7.3).

Table 7.2 Early and late complications after surgery.

\begin{tabular}{lccc}
\hline & SADI & Gastric bypass & p-value \\
& $\mathbf{n ~ ( \% )}$ & $\mathbf{n}(\%)$ & \\
\hline Anastomotic leak & $1(3.1)$ & $3(9.4)$ & 0.306 \\
Bleeding & $1(3.1)$ & $0(0)$ & 0.500 \\
Emergency care visits & $5(15.6)$ & $8(25)$ & 0.268 \\
Emergency operations & $2(6.3)$ & $3(9.4)$ & 0.500 \\
Defecation problems & $11(34.4)$ & $4(12.5)$ & $\mathbf{0 . 0 3 7}$ \\
Internal herniation & $0(0)$ & $2(6.3)$ & 0.246 \\
\hline
\end{tabular}

Table 7.3 Vitamin deficiencies.

\begin{tabular}{llccc}
\hline & Measuring moment & SADI & Gastric bypass & p-value \\
$\mathbf{n}(\%)$ & $(\%)$ & $7(21.9)$ & 0.171 \\
\hline Vitamin B12 deficiency & Baseline & $4(12.5)$ & $7(21.9)$ & 0.053 \\
& 1 year after sleeve & $1(3.8)$ & $4(12.5)$ & 0.105 \\
& 3 months after revision & $0(0)$ & $6(18.8)$ & 0.058 \\
\hline \multirow{3}{*}{ Vitamin D deficiency } & 6 months after revision & $0(0)$ & $18(56.3)$ & 0.415 \\
\cline { 2 - 5 } & Baseline & $23(71.9)$ & $12(37.5)$ & 0.295 \\
& 1 year after sleeve & $7(21.9)$ & $9(28.1)$ & 0.062 \\
& 3 months after revision & $2(6.3)$ & $19(59.4)$ & 0.067 \\
\hline
\end{tabular}

\section{Weight loss}

The \%TWL is presented Figure 7.1. Although weight loss appears slightly higher in the SADI-group, no significant differences were found ( $p>0.05)$. Furthermore, no significant differences concerning BMI were found before and after revision. Weight regain was observed in three patients from the SADI group and nine patients in de Bypass group. 


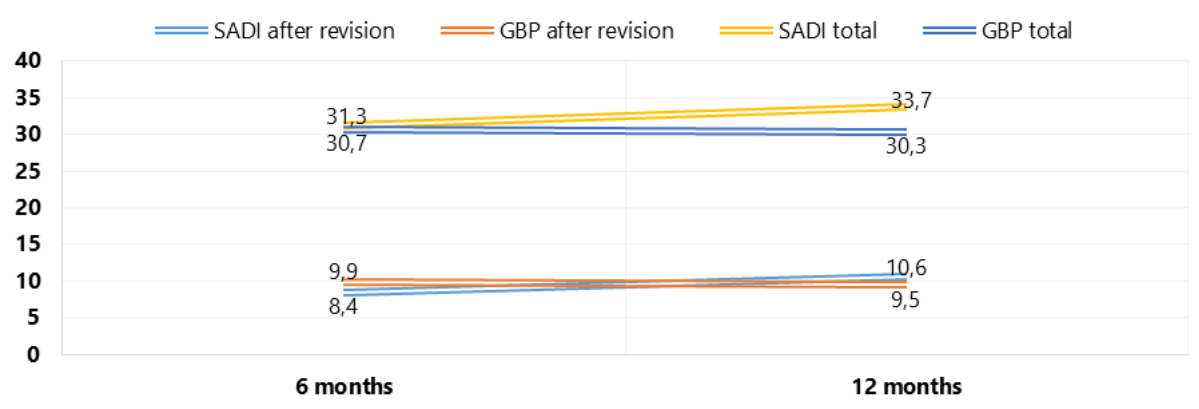

Figure 7.1 Differences in \%Total Weight Loss after revision.

SADI/GBP after revision $=$ Weight loss after revision; SADI/GBP total $=$ Total weight loss including the effect of the primary sleeve gastrectomy.

\section{Comorbidities}

Table 7.4 shows the evolution of the obesity-related comorbidities between baseline and the last moment of follow-up. Only patients who experienced an improvement or even resolvement of the comorbidity during follow-up were taken into account. Both variables include 'de novo' patients as well. No significant results were found.

Table 7.4 Reduction of comorbidities.

\begin{tabular}{|c|c|c|c|c|}
\hline & Incidence at baseline (\%) & Remissions (\%) & Improvement (\%) & $p$-value \\
\hline \multicolumn{5}{|l|}{ DM } \\
\hline$S A D I$ & 9/32 (28.1\%) & $2(22.2)$ & $1(11.1)$ & \\
\hline Bypass & $6 / 32(18.8 \%)$ & $1(16.6)$ & $3(50)$ & 0.564 \\
\hline \multicolumn{5}{|l|}{$\mathrm{HT}$} \\
\hline SADI & $14 / 32(43.8 \%)$ & $6(42.9)$ & $4(28.6)$ & \\
\hline Bypass & 10/32 (31.3\%) & $1(10)$ & $7(70)$ & 0.066 \\
\hline \multicolumn{5}{|l|}{ DL } \\
\hline SADI & $19 / 32$ (59.4\%) & $7(36.8)$ & $7(36.8)$ & \\
\hline Bypass & $17 / 32(53.1 \%)$ & $7(41.2)$ & $6(35.3)$ & 0.505 \\
\hline \multicolumn{5}{|l|}{ OSAS } \\
\hline SADI & $7 / 32$ (21.9\%) & $3(42.9)$ & $1(14.3)$ & \\
\hline Bypass & $11 / 32$ (34.4\%) & $7(63.6)$ & $2(18.2)$ & 0.471 \\
\hline \multicolumn{5}{|l|}{ GERD } \\
\hline SADI & $4 / 32(12.5 \%)$ & $2(50)$ & $1(25)$ & \\
\hline Bypass & $0 / 32(0 \%)$ & $0(0)$ & $0(0)$ & 0.273 \\
\hline \multicolumn{5}{|l|}{$\mathrm{JC}$} \\
\hline SADI & $14 / 32$ (43.8\%) & $4(28.8)$ & $7(50)$ & \\
\hline Bypass & $14 / 32(43.8 \%)$ & $7(50)$ & $3(21.4)$ & 0.206 \\
\hline
\end{tabular}

DM: diabetes mellitus; HT: hypertension; $\mathrm{DL}=$ dyslipidemia/hypercholesterolemia; OSAS=obstructive sleep apnea syndrome; GERD: gastroesophageal reflux disease; JC: joint complaints; SADI: single anastomosis duodenoileal bypass. 


\section{DISCUSSION}

This retrospective matched cohort study compared the results of SADI and GBP as revisional procedure after primary sleeve gastrectomy to achieve additional weight loss. It appeared that SADI leads to an equal remission of comorbidities, fewer vitamin deficiencies, fewer complications and a similar amount of additional weight loss compared to the GBP. Nonetheless, SADI has a longer operating time with more patients suffering from defecation problems.

Results revealed that the operating time of the SADI was significantly longer than the operating time of the GBP. This operating time may be prolonged due to the fact that these SADI-procedures were the first performed in this hospital. A completed learning curve may have a positive effect on the operating time. ${ }^{10}$ Probably the most remarkable finding is the fact that the effect of both GBP and SADI after primary sleeve gastrectomy are equal. This is remarkable since already published literature would suggest a superiority in favor of the SADI. ${ }^{11-13}$ Similar results were found in the mean BMI of both groups. Be that as it may, follow-up is limited to only one year and a higher number of patients in the Bypass group already showed weight regain. Since the follow-up in earlier studies is a few years longer, it is possible that a difference may reveal itself after a longer period of follow-up. ${ }^{11-13} A$ higher weight loss is hypothesized by the longer alimentary limb of the SADI, which may stimulate an enhanced secretion of the hormones Peptide YY and Glucagon-like Peptide-1 which are associated with increased satiety and decreased food intake. ${ }^{6,14,15}$ This hypothesis could not be reproduced in the current study, showing similar weight loss results.

The weight loss after revision is approximately one third of the total achieved weight loss, which strengthens the indication for secondary surgery after sleeve gastrectomy in certain cases.

These results contrasted with the results of comparisons between BMI per group. The SADI did not lead to significantly lower BMI values. However, results showed that subjects of the SADI group had a slightly higher initial weight. This can be explained by the indication for second surgery, which is additional weight loss. Patients of the SADI group started the project with the approach of a two-step surgery. This in contrast to patients in the Bypass group, who started assuming that the sleeve gastrectomy alone would be sufficient.

The evolution of comorbidities was similar in both groups, which indicates that both procedures (including the effect of the sleeve gastrectomy) have a comparable positive effect on the improvement of these comorbidities. This is comparable with previous literature suggesting SADI maintains a similar improvement of comorbidities as GBP. ${ }^{7,16,17}$ Nonetheless, these data should be interpreted cautiously. Due to some missing data, sums of outcome per 
variable were not consistent with their previous frequencies and the absolute number of comorbidities is low.

When analyzing the blood markers, both groups show an improvement in the lipid spectrum, without a difference between the two groups. This suggests that SADI has a similar positive effect on the lipid spectrum as GBP after primary sleeve gastrectomy, which is in accordance with previous literature. ${ }^{18}$

Significant higher levels of vitamin B12 were found in the SADI-group, which may be caused by the increased availability of intrinsic factor (IF) compared to GBP, as a larger portion of the stomach is still available to produce this glycoprotein. This consequently led to a larger amount of vitamin B12 deficiencies in the Bypass group. It should be notified that vitamin B12 levels were already significantly lower before revisional surgery, which may suggest a more aggressive supplementation regime during the SADI period compared to the GBP period in this study population.

When considering the complications after surgery, fewer anastomotic leaks, emergency care visits and emergency operations were observed in the SADI group. Meanwhile, a lot more patients complained of problems with their stools after SADI. This could be explained by the fact that the alimentary limb is longer in the SADI. ${ }^{6}$ Other studies implicate intestinal bacterial overgrowth with increased inflammation after bariatric surgery. This could possibly have more effect after a SADI procedure. Nonetheless, mechanisms are still poorly understood. Furthermore, complaints can be worse due to more malabsorption causing diarrhea due to the longer alimentary limb. Though, these symptoms are likely to disappear within months after surgery. ${ }^{19}$

Regarding the vitamin D deficiency, earlier research shows that patients who underwent GBP were often noncompliant in taking the regular vitamin supplementation. ${ }^{20}$ Furthermore, the Roux-anastomosis causes malabsorption of fat-soluble vitamins due to insufficient mixing with bile salts. ${ }^{20}$ More noncompliance and deficiencies in the Bypass group can be explained by the fact that patients who underwent surgery between 2013 and 2016 were included and the SADI was only performed from 2016 onwards. As time passed, more strict check-ups were introduced, and patients were better informed regarding vitamin supplementation. Furthermore, the SADI was considered a new surgical procedure in the Catharina hospital and results in contrast to the gold standard were not known. Therefore, the importance of supplements in this group was emphasized.

Both groups ended the follow-up period with approximately the same BMI and had an equal remission of comorbidities.

Nonetheless, this study has some considerable limitations. The first limitation is the retrospective nature of this study. Some results may be distorted due to selection bias. However, the retrospective nature also has a positive aspect, 
because true clinical circumstances are investigated and therefore, results reflect a non-research setting. ${ }^{17}$

Second limitation is the size of the study population and the duration of the follow-up. It was discussed earlier that a longer follow-up would possibly lead to more significant differences in \%TWL between groups. Furthermore, a larger study population would make it possible to give value to differences in outcome such as postoperative complications. Many of these limitations would be tackled by setting up a prospective and randomized trial.

Conclusively, SADI appears to be a promising surgical method. It seems to be a safer procedure in terms of complications considering it to be advanced bariatric surgery. The results are good (equal or better than the GBP at some points) in a short-term follow-up, however, a longer follow-up period and larger study population are needed to determine its full potential. Therefore, preferably prospective, randomized research is required. 


\section{REFERENCES}

1. Volksgezondheidenzorg.info. Overgewicht volwassenen 2017 [cited $201715^{\text {th }}$ of February]. Available from: https://www.volksgezondheidenzorg.info/onderwerp/overgewicht/cijferscontext/huidige-situatie.

2. Major $P$, Matlok $M$, Pedziwiatr $M$, Migaczewski $M$, Budzynski $P$, Stanek $M$, et al. Quality of Life After Bariatric Surgery. Obes Surg. 2015;25(9):1703-10.

3. Karamanakos SN, Vagenas K, Kalfarentzos F, Alexandrides TK. Weight loss, appetite suppression, and changes in fasting and postprandial ghrelin and peptide-YY levels after Roux-en-Y gastric bypass and sleeve gastrectomy: a prospective, double blind study. Ann Surg. 2008;247(3):401-7.

4. Dapri G, Cadiere GB, Himpens J. Superobese and super-superobese patients: 2-step laparoscopic duodenal switch. Surg Obes Relat Dis. 2011;7(6):703-8.

5. Sovik TT, Taha O, Aasheim ET, Engstrom M, Kristinsson J, Bjorkman S, et al. Randomized clinical trial of laparoscopic gastric bypass versus laparoscopic duodenal switch for superobesity. Br J Surg. 2010;97(2):160-6.

6. Sanchez-Pernaute $A$, Herrera MA, Perez-Aguirre ME, Talavera $P$, Cabrerizo L, Matia $P$, et al. Single anastomosis duodeno-ileal bypass with sleeve gastrectomy (SADI-S). One to three-year follow-up. Obes Surg. 2010;20(12):1720-6.

7. Sanchez-Pernaute A, Rubio MA, Perez Aguirre E, Barabash A, Cabrerizo L, Torres A. Singleanastomosis duodenoileal bypass with sleeve gastrectomy: metabolic improvement and weight loss in first 100 patients. Surg Obes Relat Dis. 2013;9(5):731-5.

8. Corcelles R, Boules M, Froylich D, Hag A, Daigle CR, Aminian A, et al. Total Weight Loss as the Outcome Measure of Choice After Roux-en-Y Gastric Bypass. Obes Surg. 2016;26(8):1794-8.

9. Sczepaniak JP, Owens ML, Shukla H, Perlegos J, Garner W. Comparability of weight loss reporting after gastric bypass and sleeve gastrectomy using BOLD data 2008-2011. Obes Surg. 2015;25(5):788-95.

10. Shikora SA, Kim JJ, Tarnoff ME, Raskin E, Shore R. Laparoscopic Roux-en-Y gastric bypass: results and learning curve of a high-volume academic program. Arch Surg. 2005;140(4):362-7.

11. Carmeli I, Golomb I, Sadot E, Kashtan H, Keidar A. Laparoscopic conversion of sleeve gastrectomy to a biliopancreatic diversion with duodenal switch or a Roux-en-Y gastric bypass due to weight loss failure: our algorithm. Surg Obes Relat Dis. 2015;11(1):79-85.

12. Homan J, Betzel B, Aarts EO, van Laarhoven KJ, Janssen IM, Berends FJ. Secondary surgery after sleeve gastrectomy: Roux-en-Y gastric bypass or biliopancreatic diversion with duodenal switch. Surg Obes Relat Dis. 2015;11(4):771-7.

13. Sanchez-Pernaute A, Rubio MA, Conde $M$, Arrue E, Perez-Aguirre E, Torres A. Singleanastomosis duodenoileal bypass as a second step after sleeve gastrectomy. Surg Obes Relat Dis. 2015;11(2):351-5.

14. Gibbons C, Caudwell P, Finlayson G, Webb DL, Hellstrom PM, Naslund E, et al. Comparison of postprandial profiles of ghrelin, active GLP-1, and total PYY to meals varying in fat and carbohydrate and their association with hunger and the phases of satiety. J Clin Endocrinol Metab. 2013;98(5):E847-55.

15. Webb DL, Abrahamsson N, Sundbom M, Hellstrom PM. Bariatric surgery - time to replace with GLP-1? Scand J Gastroenterol. 2017;52(6-7):635-40.

16. Gebelli JP, Gordejuela AG, Ramos AC, Nora M, Pereira AM, Campos JM, et al. Sadi-S with Right Gastric Artery Ligation: Technical Systematization and Early Results. Arq Bras Cir Dig. 2016;29Suppl 1(Suppl 1):85-90.

17. Madan AK, Orth WS, Tichansky DS, Ternovits CA. Vitamin and trace mineral levels after laparoscopic gastric bypass. Obes Surg. 2006;16(5):603-6. 
18. Kjellmo CA, Karlsson H, Nestvold TK, Ljunggren S, Cederbrant K, Marcusson-Stahl M, et al. Bariatric surgery improves lipoprotein profile in morbidly obese patients by reducing LDL cholesterol, apoB, and SAA/PON1 ratio, increasing HDL cholesterol, but has no effect on cholesterol efflux capacity. J Clin Lipidol. 2018;12(1):193-202.

19. Greenstein AJ, O'Rourke RW. Abdominal pain after gastric bypass: suspects and solutions. Am J Surg. 2011;201(6):819-27.

20. Johnson JM, Maher JW, DeMaria EJ, Downs RW, Wolfe LG, Kellum JM. The long-term effects of gastric bypass on vitamin D metabolism. Ann Surg. 2006;243(5):701-4; discussion 4-5. 



\section{CHAPTER 8}

Single Anastomosis duodenoileal bypass or GAstric bypass after primary sleeve gastrectomy (SAGA-trial): a randomized controlled trial (Study protocol)

M.R. van Wezenbeek

J.F. Smulders

J.P.J.G.M. de Zoete

M.D. Luyer

G. van Montfort

S.W. Nienhuijs

Submitted

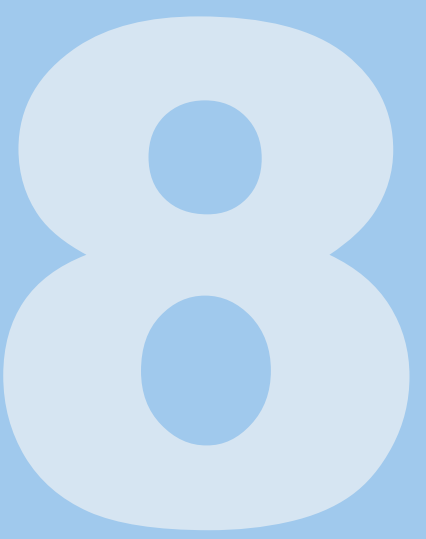




\section{ABSTRACT}

\section{Background}

To achieve additional weight loss after primary sleeve gastrectomy, Roux-en-Y gastric bypass (RYGB) is the most performed procedure, however results are disappointing. New procedures such as the Single Anastomosis Duodeno-lleal bypass (SADI) appear to be a better alternative, however there is only limited evidence to support this assumption. This article describes the study protocol of a randomized controlled trial comparing the outcomes of the RYGB and SADI after primary sleeve gastrectomy.

\section{Methods \& Design}

This is a single-centre, prospective, randomized controlled, clinical study. The trial is conducted as a superiority trial to demonstrate that SADI is better than RYGB after primary sleeve gastrectomy. The primary endpoint of this study will be the additional weight loss in terms \%EWL after 2 years. Secondary outcome measurements for this study will be weight loss expressed with different parameters, the comparison of peri- and postoperative complications, reduction of obesity-related comorbidities, biochemical changes, gut flora changes, convalescence, adherence to the bariatric program, dumping syndrome complaints and quality of life. Total follow-up of the study will be two years. The aim is to include fifty patients (age between 18 and 65), with a body mass index between 35 and $60 \mathrm{~kg} / \mathrm{m}^{2}$ for whom a secondary procedure after primary sleeve gastrectomy is indicated.

\section{Discussion}

This study aims to provide important data on the impact of RYGB and SADI after primary sleeve gastrectomy in terms of weight loss and many other parameters. 


\section{BACKGROUND}

Morbid obesity remains a global burden, for which bariatric surgery still appears to be the most effective therapy. It is known to achieve long-term weight loss, a risk reduction of cardiovascular morbidity and an improvement in both lifestyle and any known obesity-related comorbidities such as diabetes mellitus type 2 and hypertension. ${ }^{1-3}$

Bariatric has become increasingly popular over the last years. In 2011, over 340,000 bariatric procedures were performed worldwide of which adjustable gastric banding (AGB), sleeve gastrectomy (SG) and Roux-en- $Y$ gastric bypass (RYGB) were performed most frequently. ${ }^{4}$ AGB has been decreasing in popularity over the last years due to poor long-term results. ${ }^{5}$ The RYGB is currently still considered the best surgical option for the treatment of morbid obesity, although seems to be surpassed by the SG in numbers. ${ }^{6}$ The sleeve gastrectomy was first described by Marceau et al. in the late 1990's as part of a biliopancreatic diversion with duodenal switch (BPD/DS) and was later popularized by Gagner et al. as standalone procedure. ${ }^{7-9}$ SG is a bariatric procedure based on a restriction of food intake by removal of the gastric fundus, which is the predominant area for the production of ghrelin. ${ }^{10}$ Ghrelin has been described as a hunger regulating peptide hormone. ${ }^{11} \mathrm{SG}$ is known to achieve good short- and medium term weight loss results, however long-term results are scarce. ${ }^{12}$ Nevertheless a long-term drawback of SG is weight regain, of which recently reported percentages are between 5.4-29.5\%, often necessitating another bariatric procedure to achieve additional weight loss or prevent further weight regain. ${ }^{13-16}$ Current revision rates of SG are between $5.5-11 \%$, taking into account that these rates are not only for weight regain, but also other complications such as gastroesophageal reflux disease, dysphagia and insufficient weight loss. ${ }^{15-17}$ These rates are expected to increase over the next years.

There are several options for secondary surgery after SG: re-sleeve gastrectomy, mini gastric bypass, RYGB, BPD/DS or single anastomosis Duodenoileal bypass (SADI). RYGB and BPD/DS appear to be the most preferable as secondary procedure. ${ }^{14,16-19}$ However, the more recently introduced SADI also appears to have promising results in terms of weight loss. ${ }^{20}$ The SADI is a derivative of the primary procedure SADI-S, which can be described as a simplified duodenal switch and has demonstrated to have satisfactory short- and medium-term results. $^{21}$

In a SADI, a duodenojejunal anastomosis is created, keeping the pyloric valve intact. The common loop is measured from Bauhin's valve (approximately $250 \mathrm{~cm})$. 
In a RYGB, the pyloric valve is excluded from the food trajectory. By creating a small gastric pouch, after which a gastrojejunostomy is created. Keeping the pyloric valve intact reduces the occurrence of dumping syndrome but may potentially maintain complaints such as reflux or dysphagia. Therefore, RYGB is preferred as secondary procedure for complaints of gastro-esophageal reflux or dysphagia. $^{22}$

In case of insufficient weight loss or weight regain, SADI appears to achieve more additional weight loss compared to RYGB, however may be associated with more nutritional deficiencies and complaints such as fatty diarrhea and (persisting) complaints of reflux or dysphagia.

Evidence on which of these two procedures is preferred for achieving the most additional weight loss after sleeve gastrectomy is scarce. Results provided are of a retrospective nature with small groups, long-term results are lacking and thus conclusions lack hard evidence.

In the recently published study by Torres et al., conversion to SADI appears to produce similar results as conversion to BPD/DS, however the intra- and postoperative complication rate is lower. ${ }^{19,20}$ Again, evidence is scarce but the first results appear promising and deserve further investigation. Furthermore, no comparison was made between the SADI and any other bariatric procedure as secondary procedure after sleeve gastrectomy.

Therefore, this randomized controlled trial aims to determine whether SADI is a feasible alternative to the RYGB, the current gold standard, as secondary procedure for achieving additional weight loss after SG.

Indications for inclusion will be either weight regain, insufficient weight loss or a planned secondary procedure after primary sleeve gastrectomy.

Primary outcome of this study will be weight loss in terms of percentage excess weight loss (\%EWL) 2 years after surgery.

Secondary outcome measurements for this study will be other weight loss parameters, peri- and postoperative complications, reduction of obesity-related comorbidities, biochemical changes, gut flora changes, adherence to the bariatric program, dumping syndrome complaints and quality of life.

\section{METHODS AND DESIGN}

This single-centre, prospective, randomized controlled, clinical study will be conducted at the Catharina Hospital, in Eindhoven, the Netherlands in both a clinical and an outpatient clinic setting. In- and exclusion criteria for all participants are displayed in Table 8.1. Total follow-up of participants will be two years. 
Table 8.1 Inclusion and exclusion criteria.

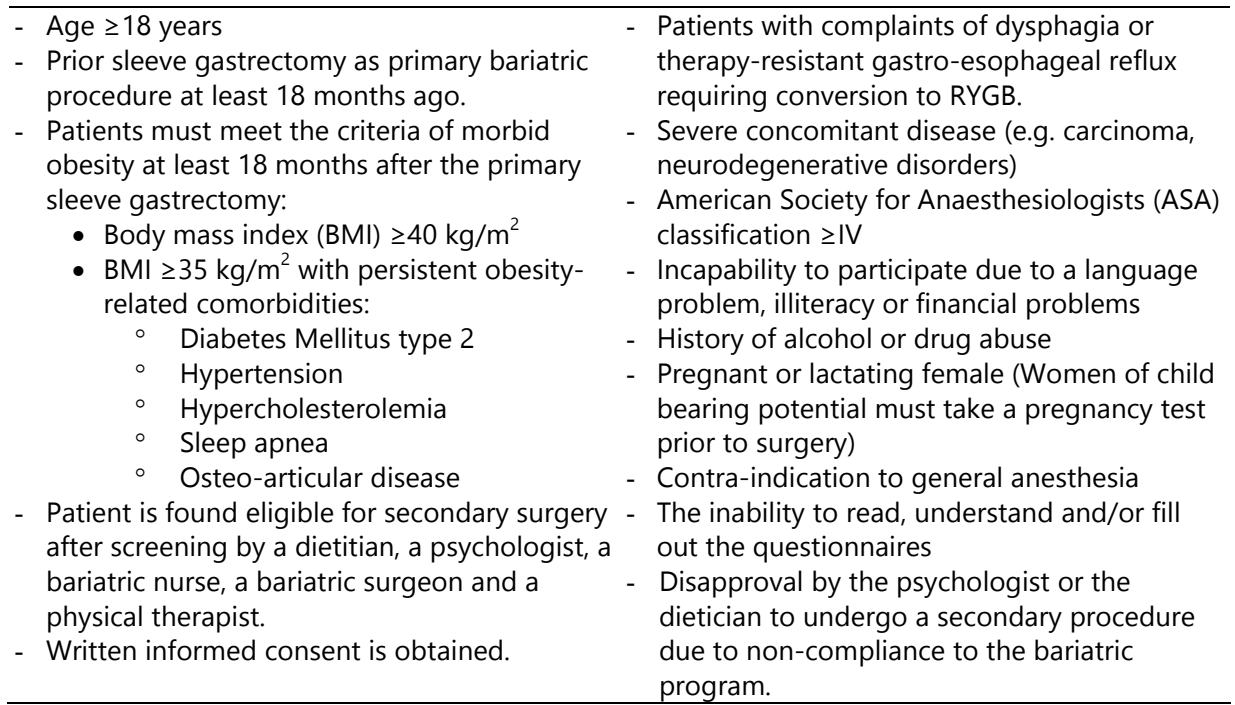

\section{Primary objective}

The primary endpoint of this study will be weight loss, which will be expressed by the percentage of total excess weight loss (\%EWL) after a minimal follow-up period of 2 years. Total excess weight is defined as the difference between the weight before primary surgery and the highest healthy weight, which is at a body mass index (BMI) of $25 \mathrm{~kg} / \mathrm{m}^{2}$. \%EWL is calculated as (pre-sleeve BMI current BMI) / (pre-sleeve -25$)$ * $100 \%$.

\section{Secondary objectives}

There will be a number of secondary endpoints which are listed below:

1. To compare the operative time and textbook-outcome (discharge <2 days and no complications per-operative and in the first 30-days).

Included postoperative complications are surgical complications (anastomotic leakage, bleeding, incidental gastrointestinal perforation), infectious complications (intra-abdominal abscess, pneumonia, urinary tract infection, wound infection, gastroenteritis), major cardiovascular events, venous thromboembolism, anastomotic stenosis, marginal ulcer and postoperative ileus. All postoperative complications are also scored according to the Clavien-Dindo scale. ${ }^{23} \mathrm{~A}$ complication is considered a serious adverse event when the Clavien-Dindo equals $3 a$ (A complication 
requiring surgical, endoscopic or radiological intervention not under general anesthesia) or higher.

2. Since several parameters are used worldwide to express the amount of weight loss after bariatric surgery of which some are used to further evaluate additional weight loss:

- Percentage excessive weight loss after conversion (\%EWL after conversion): (BMI at revision - BMI at follow-up) / (BMI at revision - 25) * $100 \%$

- Total change in BMI (cBMI): pre-sleeve BMI - current BMI

- Change in BMI after conversion (CBMI after conversion): BMI at revision - current BMI

- Percentage total weight loss (\%TWL): (pre-operative weight - current weight) / (pre-operative weight) * $100 \%$

- Percentage total weight loss after conversion (\%TWL after conversion): (weight at revision - weight at follow-up) / (weight at revision) * $100 \%$

3. To evaluate the improvement or even resolution of any present obesityrelated comorbidities (diabetes mellitus type 2, hypertension, dyslipidemia, sleep apnea, osteo-articular disease). All medication use and changes in medication doses will be documented every follow-up moment. Furthermore, laboratory findings will be obtained and analyzed preoperatively and every 6 months after surgery to ensure they are in normal range.

4. To evaluate any biochemical changes after surgery by means of drawing blood. (Hemoglobin, hematocrit, MCV, ASAT, ALAT, bilirubin, creatinine, sodium, potassium, calcium, phosphate, albumin, lipid spectrum, HbA1c, insulin, C-peptide, methyl malonic acid, vitamin B12, iron, ferritin, folic acid, magnesium, vitamin $D$, vitamin A, vitamin B1, vitamin B6).

5. To evaluate complaints and/or complications $>30$ days including dysphagia, gastro-esophageal reflux disease (GERD), defecation problems, the need for re-do surgery for either weight regain, insufficient weight loss or medical complaints, vitamin deficiencies using the biochemical results, and internal herniation GERD will be evaluated by using the Frequency Scale for Symptoms of GERD (FSSG) and dysphagia will be evaluated using a questionnaire designed by Suter et al. assessing food tolerance after bariatric surgery. ${ }^{24,25}$ Patients will be asked to complete this questionnaire on a yearly basis during the study period.

In addition, patients will report on their defecation pattern including frequency and consistency of the stool using the Bristol Stool Chart.

All diagnoses and frequencies of internal herniation, vitamin deficiencies, and weight loss complaints will be scored. 
6. To evaluate (changes in) quality of life (QoL), objectified by the Research and Development-36 (RAND-36) questionnaire. Additionally, convalescence after surgery will be evaluated with the Surgical Recovery Scale (SRS) questionnaire to evaluate the general postoperative recovery.

7. To evaluate the adherence of the included patients to the bariatric program by keeping records of attendance to the outpatient clinic visits. Furthermore, a record of the number of unscheduled visits will be kept.

8. To evaluate the occurrence of dumping syndrome (DS) complaints. DS will be diagnosed using the Dumping Syndrome Rating Scale (DSRS).

9. To evaluate the possible postoperative changes in the gut flora, providing new opportunities to understand the relationship of the gastrointestinal tract to obesity as well as new therapeutic strategies. Stools will be investigated at four different moments: 1 . Before surgery, 2. Four weeks after surgery 3 . Three months after surgery and 4 . One year after surgery.

\section{Interventions}

All procedures will be performed by experienced bariatric surgeons who perform at least 100 bariatric procedures every year.

All patients will be treated peri-operatively according to the hospital-adjusted fast track protocol as earlier described in another manuscript. ${ }^{26}$ Patients will undergo either conversion from primary sleeve gastrectomy to SADI or RYGB.

Laparoscopic conversion of sleeve gastrectomy to single anastomosis duodenoileal bypass

After achieving pneumoperitoneum by placing a Veress needle in the left upper quadrant of the abdomen, trocars (normally between five and seven) are placed according to the preference of the surgeon.

The procedure is initiated by any adhesiolysis when necessitated using a dissection device. Then, the duodenum is identified, stapled and transected about $2 \mathrm{~cm}$ distal to the pyloric valve at the level of the gastroduodenal artery. The ileocecal valve is identified and proximal of it, $300 \mathrm{~cm}$ of small intestine are measured backwards, making the common channel. The loop is ascended antecolically and an end-to-side duodeno-ileal anastomosis is constructed using a linear stapler (EndoGIA, Covidien) followed by a running suture (V-Loc, Covidien). The length of the alimentary loop will be measured. The mesenteric defects will not be closed routinely. A leak test of the anastomosis is performed by applying methylene blue over a gastric tube. The procedure is finished by removal of the trocars under sight and closure of the skin. 
Laparoscopic conversion of sleeve gastrectomy to Roux-en-Y gastric bypass

After achieving pneumoperitoneum by placing a Veress needle in the left upper quadrant of the abdomen, trocars (normally between five and seven) are placed according to the preference of the surgeon.

The procedure is initiated by any adhesiolysis when necessitated using a dissection device. Then the procedure continues with perigastric dissection of the lesser curvature to enter the omental bursa. Next, a linear $45 \mathrm{~mm}$ endostapler (EndoGIA, Covidien) is inserted in the created opening to horizontally transect the stomach to create a gastric pouch. In case of sleeve dilatation, the pouch is resized vertically towards the angle of His, along a $34 \mathrm{Fr}$ gastric tube. The small bowel is brought up towards the newly created gastric pouch at a length of $60-80 \mathrm{~cm}$ from the point of Treitz. At this point, a gastrojejunostomy is constructed using a linear stapler (EndoGIA, Covidien) followed by a running suture (V-Loc, Covidien). Next, an enteroenterostomy is created using a linear stapler (EndoGIA, Covidien) and a running suture between the small bowel proximal to the gastrojejunostomy and a point of small bowel $150 \mathrm{~cm}$ distally from the gastrojejunostomy. The length of the common channel will be measured. The mesenteric defect will be closed routinely. A leak test of the proximal anastomosis is performed by applying methylene blue over a gastric tube.

The procedure is finished by removal of the trocars under sight and closure of the skin.

\section{Allocation}

When it is not technically possible to perform SADI during surgery, RYGB will be performed instead and vice versa. These patients will remain in their initial allocated group for statistical analysis in accordance with the intention-to-treat principle.

When neither a SADI nor RYGB is technically possible, another bariatric procedure will be performed or the procedure will be cancelled as the surgeon's wishes and the patient will be excluded from the study.

\section{Participant timeline}

An overview of the enrolment, interventions, assessments and visits for participants is shown in Figure 8.1. Most of the assessments are during the visits at the outpatient clinic. 


\section{Sample size and recruitment}

Results on \%EWL for SADI after primary sleeve gastrectomy or SADI-S are still scarce, but are currently reported to be between $72 \%$ and $98 \%{ }^{20,27}$ According to a recent report by Carmeli et al comparing BPD/DS and RYGB as secondary procedure after sleeve gastrectomy, the mean \%EWL after SADI is $80 \%$ and the mean \%EWL after RYGB is $65.5 \%{ }^{18}$

Combining these findings combined with the fact that the SADI is considered a simplified version of the BPD/DS, it is hypothesized that the mean \%EWL after SADI will be approximately $80 \%{ }^{28}$ Mean \%EWL after RYGB is set at $65.5 \%$, based on the reports by Carmeli et al.

Current sample size was calculated according to a continuous outcome superiority design. The significance level (alpha) was set at 0.05 and the power (1-beta) was set at 0.90 . Mean outcomes are $65.5 \%$ in the control group and $80 \%$ in the experimental group. The standard deviation was set at $15 \%$. Using these settings, sample size calculation shows that a total sample size of 46 patients is required, which means 23 patients per group. Taking a 10\% dropout into account, the aim is to include 50 patients.

In this, well over 3,000 primary sleeve gastrectomies have been performed up to 2017 and the annual number is still increasing. A number of these patients are expected to return over the next years with complaints of either weight regain, insufficient weight loss or to assess the indication for a planned secondary procedure. ${ }^{13-15}$ These patients will be considered for inclusion in the current study when revisional surgery is indicated to achieve additional weight loss.

Considering the weight regain percentages reported in previous literature (5.4\%-29\%) within five years, it can be expected that at least 50-100 patients will return annually. An inclusion period of 24 months should be sufficient to include the number of patients needed. A ten percent dropout rate is taken into account.

\section{Randomisation}

Patients willing to enter the bariatric program as well as the current study, will be randomized at the outpatient clinic by the attending physician. Computerized randomization for allocation of the treatment group will take place through the trial website after verification that all inclusion criteria and none of the exclusion criteria have been met. Then, written informed consent will be obtained by the attending physician. 

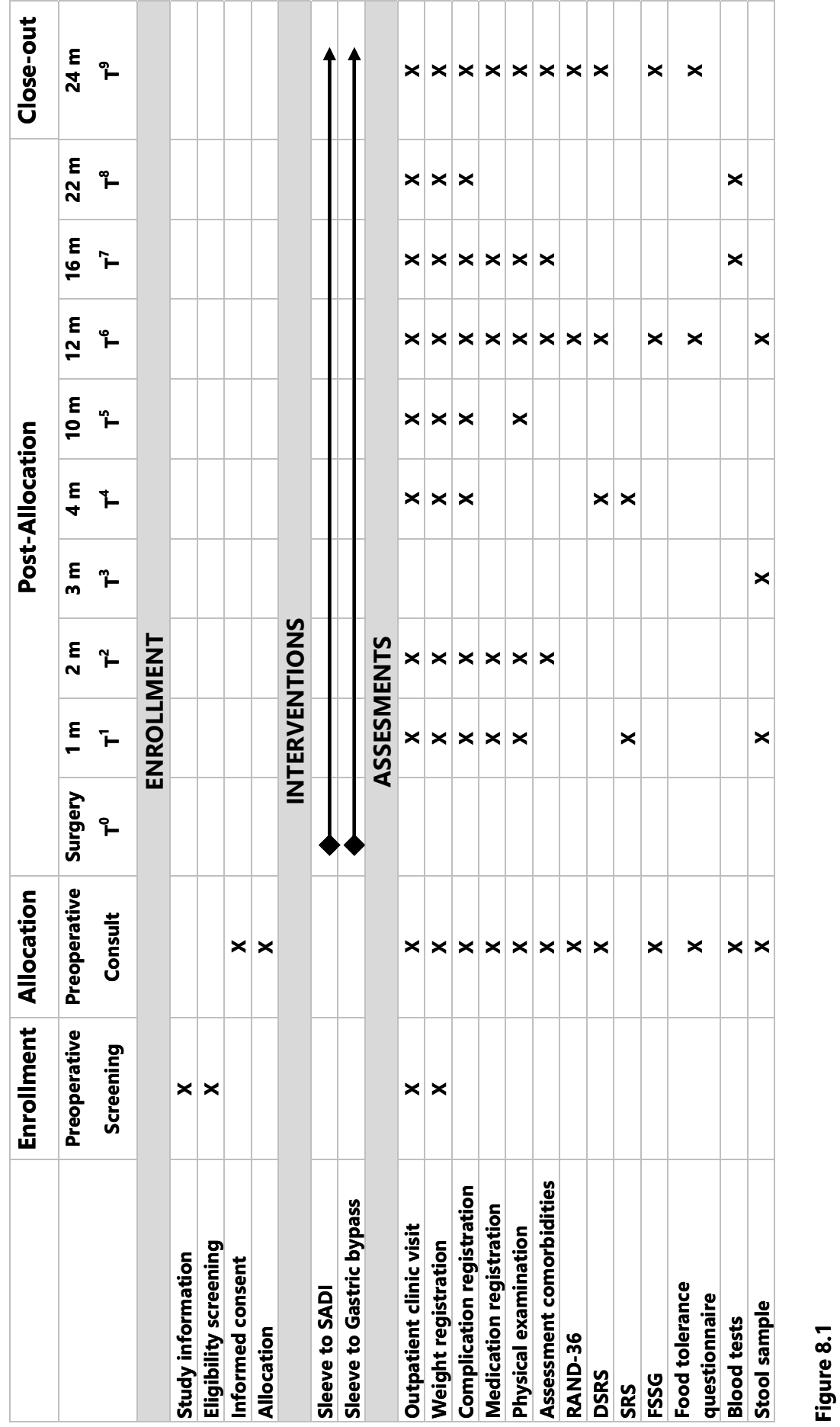
Randomisation will take place at the moment of the scheduling of the procedure and the patient will be informed immediately on the outcome of the randomization. Randomization will be performed by one of the bariatric surgeons, who will be the only ones who will have access to the trial website besides the main investigators. Participants will be given as much time as they desire to consider their decision. In case of extra questions, an independent doctor is available to answer them. Patients are also asked for their consent to keep record of their medical data after the 2-year study period. This is already a standard procedure at the Obesity Centre. These data can then be used for long-term follow up studies.

No blinding is performed.

\section{Adherence}

To evaluate the adherence of the included patients to the bariatric program, records will be kept of whether or not patients have attended the outpatient clinic visits. Reminders will be sent to patients both by phone and by mail to increase the adherence to the study protocol.

It is believed that proper follow-up of patients after bariatric surgery will increase the likelihood of sufficient weight loss. Therefore, a record will be kept of the number of missed outpatient clinic appointments.

\section{Statistical analyses}

All data will be handled confidentially and anonymously. All patients are given a subject identification code which will be linked to the data of the subject in case it is found necessary to trace data to an individual subject. The code is not based on the patient's initials or birth date. The code is based on the order in which the patient is enrolled in the study.

The key to the code is safeguarded by the principal investigator. The handling of personal data is done in accordance with the Dutch Personal Data Protection Act.

All data will be stored on CCM, the study section of the eHealth environment of BePatient, an online patient platform used by the Catharina hospital's Obesity Center. This section is not accessible by any of our patients. All data is stored securely and can only be accessed with one code which is only available to the principal investigator. One file with a unique study number will be provided per patient.

SPSS version 24, for Windows (SPSS Inc, Chicago, IL, USA), will be used for the management and analysis of all data. 
The data of the randomized patients are analysed according to the intentionto-treat principle. Total follow-up will be two years after surgery. The regular post bariatric program will be continued after two years up till five years after surgery.

Continuous variables that are normally distributed will be presented as a mean with standard deviations. Skewed continuous variables will be presented as median with interquartile ranges (IQR). Variables measured on a nominal or ordinal scale are summarized as an amount (n) with a percentage as total of the investigated group.

Differences I $\mathrm{n}$ baseline characteristics, as well as differences in operative time, hospital stay will be univariately tested. Continuous, normally distributed variables are tested with the unpaired Student's $t$-test. Non-parametric MannWhitney $U$ test is performed is case of skewed continuous variables. In case of nominal or ordinal variables, Chi-square test (or Fisher's exact test, when appropriate) is performed. Differences in overall mortality rates and diseasespecific mortality rates over time between RYGB and SADI will be evaluated with Kaplan-Meier analysis and tested with the log rank test. Differences in continuous measurements over time (SRS outcome, DSRS outcome and RAND36 outcome), \%TWL, \%EWL, CBMI and biochemical changes between RYGB and SADI will be expressed as mean ( \pm standard deviation) or median (IQR).

Differences in rates or proportions over time (proportion of patients with \%EWL $\geq 50$, peri- and postoperative morbidity rates, readmission rates, short- and long-term complication rates, improvement/resolution rates of obesity-related comorbidities and the need for re-do surgery between RYGB and SADI are expressed as (cumulative) incidence rates or (cumulative) incidence density rates. Reasons/causes for secondary surgery are analysed in a similar way (e.g. psychological reason, technical reasons).

A subgroup analysis is performed similarly to the method as described above. Subgroups will be patients with weight regain versus patients with insufficient weight loss after primary SG.

\section{Monitoring}

The investigator will submit a summary of the progress of the trial to the accredited Medical Ethics Research Committee (MERC) once a year. Information will be provided on the date of inclusion of the first subject, numbers of subjects included and numbers of subjects that have completed the trial, serious adverse events/ serious adverse reactions, other problems, and amendments.

When a serious adverse event (SAE) occurs, the principal investigator will be notified within 24 hours by email or telephone. The investigator will report the 
SAEs through the web portal ToetsingOnline to the accredited MERC that approved the protocol, within 15 days after the investigator has first knowledge of the serious adverse events.

An SAE not listed above will be recorded in an overview list that will be submitted periodically to the MERC (once every 6 months).

All involved physicians will periodically be asked to report any adverse events caused by the current study protocol. These events will be listed and discussed. The monitoring board can ask for a full report on any adverse event in order to discuss a specific event. A copy of this report will be sent to the central ethics board and to the involved physicians.

All deceased patients will be evaluated by the safety board for cause of death and possible trial related serious adverse effects. Every death will be reported to the central ethics board and the local ethics board.

\section{Ethical considerations}

The current study will be conducted according to the principles of the Declaration of Helsinki (eighth version, October 2013) and in accordance with the Medical Research Involving Human Subjects Act.

In accordance with the legal requirements in the Netherlands an insurance policy is closed. This insurance provides cover for damage to research subjects through injury or death caused by the study.

All patients will be properly informed and a written informed consent is obtained before they will be included in the trial. They all consent to the fact that their medical data will be used anonymously for publication.

An approval of the hospital's MERC is currently in progress.

\section{DISCUSSION}

As discussed earlier, sleeve gastrectomy is currently one of the most performed procedures with good long-term results. ${ }^{29}$ The downfall is an increasing amount of failure in terms of acceptable weight loss. Often, patients show weight regain and a great portion of those patients undergo revision. ${ }^{13,14,16}$ There is very limited data on the long-term follow-up after sleeve gastrectomy, but since the absolute number of sleeve gastrectomies is rapidly increasing, it is expected that the number of patients necessitating revisional surgery will increase too. Nowadays, RYGB is considered the golden standard for revision of a failed sleeve gastrectomy, however more data with disappointing results have recently been published. ${ }^{30,31}$ 
Therefore, new alternatives are being explored. The first data on the SADI appear promising, but require further investigation. ${ }^{20}$

This trial aims to answer whether SADI achieves superior weight loss (in terms of percentage total body weight loss) results compared to RYGB after primary sleeve gastrectomy. Furthermore, this study aims to clarify a number of other outcomes as described in the Methods section of this manuscript.

For example, there is very little known about the biochemical changes after SADI. Since SADI is a malabsorptive procedure, vitamin deficiencies are likely to occur.

Standard issues such as postoperative complications, improvement of obesityrelated comorbidities, convalescence and quality of life are investigated as well. An issue important to patients may be functional complaints such as dumping syndrome, which is one of the functional concerns after RYGB. It is expected that these complaints will be significantly less after SADI, mainly due to the preservation of the pyloric sphincter, but to our knowledge, no evidence is currently available on this issue.

More and more evidence shows that good postoperative support is associated with an increase in weight loss and an improvement in comorbidities. ${ }^{32,33}$ Therefore, adherence to the program will be taken into account in this study as well.

A relative new field within weight reduction therapy are changes in the gut microbiota. It has been shown that the gut microbiome may change after bariatric surgery and affect weight loss, but more data is needed on this subject and data on changes after a revisional bariatric procedure is scarce. ${ }^{34}$

In conclusion, the aim of this study is to investigate whether SADI is a superior alternative to RYGB as revisional procedure after primary sleeve gastrectomy in terms of additional weight loss. Additionally, this study attempts to further discover many unknown elements of the SADI. 


\section{REFERENCES}

1. Ribaric G, Buchwald JN, McGlennon TW. Diabetes and weight in comparative studies of bariatric surgery vs conventional medical therapy: a systematic review and meta-analysis. Obes Surg. 2014;24(3):437-55.

2. Sjostrom L. Bariatric surgery and reduction in morbidity and mortality: experiences from the SOS study. Int J Obes (Lond). 2008;32 Suppl 7:S93-7.

3. Sjostrom L, Lindroos AK, Peltonen M, Torgerson J, Bouchard C, Carlsson B, et al. Lifestyle, diabetes, and cardiovascular risk factors 10 years after bariatric surgery. N Engl J Med. 2004; 351(26):2683-93.

4. Buchwald H, Oien DM. Metabolic/bariatric surgery worldwide 2011. Obes Surg. 2013;23(4): 427-36.

5. Aarts EO, Dogan K, Koehestanie P, Aufenacker TJ, Janssen IM, Berends FJ. Long-term results after laparoscopic adjustable gastric banding: a mean fourteen year follow-up study. Surg Obes Relat Dis. 2014;10(4):633-40.

6. Sjostrom L, Narbro K, Sjostrom CD, Karason K, Larsson B, Wedel H, et al. Effects of bariatric surgery on mortality in Swedish obese subjects. N Engl J Med. 2007;357(8):741-52.

7. Gumbs AA, Gagner M, Dakin G, Pomp A. Sleeve gastrectomy for morbid obesity. Obes Surg. 2007;17(7):962-9.

8. Marceau P, Hould FS, Simard S, Lebel S, Bourque RA, Potvin M, et al. Biliopancreatic diversion with duodenal switch. World J Surg. 1998;22(9):947-54.

9. Regan JP, Inabnet WB, Gagner M, Pomp A. Early experience with two-stage laparoscopic Roux-en-Y gastric bypass as an alternative in the super-super obese patient. Obes Surg. 2003;13(6):861-4.

10. Langer FB, Reza Hoda MA, Bohdjalian A, Felberbauer FX, Zacherl J, Wenzl E, et al. Sleeve gastrectomy and gastric banding: effects on plasma ghrelin levels. Obes Surg. 2005;15(7): 1024-9.

11. Masuda $Y$, Tanaka T, Inomata N, Ohnuma N, Tanaka S, Itoh Z, et al. Ghrelin stimulates gastric acid secretion and motility in rats. Biochem Biophys Res Commun. 2000;276(3):905-8.

12. Brethauer SA, Hammel JP, Schauer PR. Systematic review of sleeve gastrectomy as staging and primary bariatric procedure. Surg Obes Relat Dis. 2009;5(4):469-75.

13. Liu SY, Wong SK, Lam CC, Yung MY, Kong AP, Ng EK. Long-term Results on Weight Loss and Diabetes Remission after Laparoscopic Sleeve Gastrectomy for A Morbidly Obese Chinese Population. Obes Surg. 2015;25(10):1901-8.

14. Cesana G, Uccelli M, Ciccarese F, Carrieri D, Castello G, Olmi S. Laparoscopic re-sleeve gastrectomy as a treatment of weight regain after sleeve gastrectomy. World J Gastrointest Surg. 2014;6(6):101-6.

15. Langer FB, Shakeri-Leidenmuhler S, Bohdjalian A, Schoppmann S, Zacherl J, Felberbauer FX, et al. Strategies for weight regain after sleeve gastrectomy. Surg Laparosc Endosc Percutan Tech. 2010;20(3):159-61.

16. van Rutte PW, Smulders JF, de Zoete JP, Nienhuijs SW. Indications and short-term outcomes of revisional surgery after failed or complicated sleeve gastrectomy. Obes Surg. 2012; 22(12):1903-8.

17. Weiner RA, Theodoridou $\mathrm{S}$, Weiner $\mathrm{S}$. Failure of laparoscopic sleeve gastrectomy--further procedure? Obes Facts. 2011;4 Suppl 1:42-6.

18. Carmeli I, Golomb I, Sadot E, Kashtan H, Keidar A. Laparoscopic conversion of sleeve gastrectomy to a biliopancreatic diversion with duodenal switch or a Roux-en-Y gastric bypass due to weight loss failure: our algorithm. Surg Obes Relat Dis. 2015;11(1):79-85.

19. Homan J, Betzel B, Aarts EO, van Laarhoven KJ, Janssen IM, Berends FJ. Secondary surgery after sleeve gastrectomy: Roux-en-Y gastric bypass or biliopancreatic diversion with duodenal switch. Surg Obes Relat Dis. 2015;11(4):771-7. 
20. Sanchez-Pernaute A, Rubio MA, Conde M, Arrue E, Perez-Aguirre E, Torres A. Singleanastomosis duodenoileal bypass as a second step after sleeve gastrectomy. Surg Obes Relat Dis. 2015;11(2):351-5.

21. Sanchez-Pernaute A, Rubio MA, Perez Aguirre E, Barabash A, Cabrerizo L, Torres A. Singleanastomosis duodenoileal bypass with sleeve gastrectomy: metabolic improvement and weight loss in first 100 patients. Surg Obes Relat Dis. 2013;9(5):731-5.

22. Parmar CD, Mahawar KK, Boyle M, Schroeder N, Balupuri S, Small PK. Conversion of Sleeve Gastrectomy to Roux-en-Y Gastric Bypass is Effective for Gastro-Oesophageal Reflux Disease but not for Further Weight Loss. Obes Surg. 2017;27(7):1651-8.

23. Dindo D, Demartines $N$, Clavien PA. Classification of surgical complications: a new proposal with evaluation in a cohort of 6336 patients and results of a survey. Ann Surg. 2004;240(2): 205-13.

24. Kusano M, Shimoyama $Y$, Sugimoto S, Kawamura O, Maeda M, Minashi K, et al. Development and evaluation of FSSG: frequency scale for the symptoms of GERD. J Gastroenterol. 2004; 39(9):888-91.

25. Suter M, Calmes JM, Paroz A, Giusti V. A new questionnaire for quick assessment of food tolerance after bariatric surgery. Obes Surg. 2007;17(1):2-8.

26. van Wezenbeek MR, Pouwels SJ, Buise MP, Smulders JF, Nienhuijs SW, van Montfort G. Conventional versus fast track anaesthesia in an unselected group of patients undergoing revisional bariatric surgery. International Journal of Surgery Open. 2016;1:22-7.

27. Sanchez-Pernaute A, Herrera MA, Perez-Aguirre ME, Talavera $P$, Cabrerizo $L$, Matia $P$, et al. Single anastomosis duodeno-ileal bypass with sleeve gastrectomy (SADI-S). One to three-year follow-up. Obes Surg. 2010;20(12):1720-6.

28. Sanchez-Pernaute A, Rubio MA, Cabrerizo L, Ramos-Levi A, Perez-Aguirre E, Torres A. Singleanastomosis duodenoileal bypass with sleeve gastrectomy (SADI-S) for obese diabetic patients. Surg Obes Relat Dis. 2015;11(5):1092-8.

29. Gadiot RP, Biter LU, van Mil S, Zengerink HF, Apers J, Mannaerts GH. Long-Term Results of Laparoscopic Sleeve Gastrectomy for Morbid Obesity: 5 to 8-Year Results. Obes Surg. 2017; 27(1):59-63.

30. Malinka T, Zerkowski J, Katharina I, Borbely YM, Nett P, Kroll D. Three-Year Outcomes of Revisional Laparoscopic Gastric Bypass after Failed Laparoscopic Sleeve Gastrectomy: a CaseMatched Analysis. Obes Surg. 2017;27(9):2324-30.

31. van Wezenbeek MR, van Oudheusden TR, de Zoete JP, Smulders JF, Nienhuijs SW. Conversion to Gastric Bypass After Either Failed Gastric Band or Failed Sleeve Gastrectomy. Obes Surg. 2017;27(1):83-9.

32. Livhits M, Mercado C, Yermilov I, Parikh JA, Dutson E, Mehran A, et al. Is social support associated with greater weight loss after bariatric surgery?: a systematic review. Obes Rev. 2011;12(2):142-8.

33. Schwoerer A, Kasten K, Celio A, Pories W, Spaniolas K. The effect of close postoperative follow-up on co-morbidity improvement after bariatric surgery. Surg Obes Relat Dis. 2017; 13(8):1347-52.

34. Anhe FF, Varin TV, Schertzer JD, Marette A. The Gut Microbiota as a Mediator of Metabolic Benefits after Bariatric Surgery. Can J Diabetes. 2017;41(4):439-47. 


\section{PART TWO}

Early complications in bariatric surgery 



\section{CHAPTER 9}

\section{Predictors for the occurrence of major complications after primary Roux-en-Y gastric bypass surgery}

M.R. van Wezenbeek

J.F. Smulders

M.D. Luyer

G. van Montfort

F.J.G. Vanhimbeeck

S.W. Nienhuijs

Minerva Chirurgica. 2016;71(5):286-92

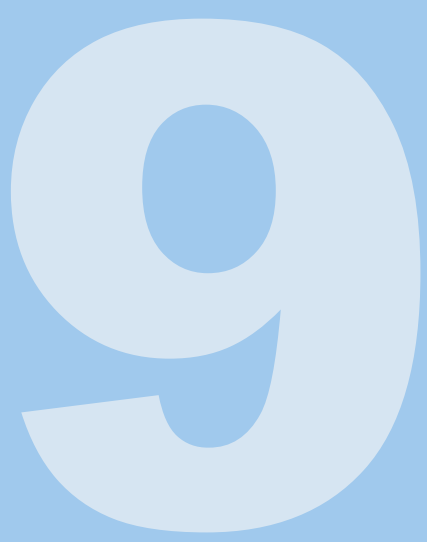




\section{ABSTRACT}

\section{Background}

The risk of developing postoperative complications after primary Roux-en- $Y$ gastric bypass (RYGB) is relatively low. Nevertheless, postoperative complications can have serious consequences in terms of severe morbidity and health care costs. Identification of potential predictors is useful for further reduction of the postoperative complication rate.

\section{Methods}

This retrospective study included all patients undergoing primary RYGB between January 2010 and December 2013, using data from a prospectively collected database. Patients' characteristics, operative details and perioperative outcome were analyzed.

\section{Results}

A total of 773 patients ( $14.5 \%$ male) were included for analysis, with a mean age of $42.1 \pm 10.4$ years and a mean Body Mass Index of $42.8 \pm 4.3 \mathrm{~kg} / \mathrm{m}^{2}$. A total of $66(8.5 \%)$ direct postoperative complications occurred. Clavien-Dindo grade $3 a$ and higher occurred in 55 patients. Univariate analysis identified age $(p=0.013)$, gender $(p=0.017), \mathrm{BMI}$ over $50 \mathrm{~kg} / \mathrm{m}^{2} \quad(p=0.096)$, hypertension $(p=0.099)$, chronic obstructive pulmonary disease $(p=0.002)$ and previous upper gastrointestinal surgery $(p=0.095)$ as potential predictors. Multivariate logistic regression analysis showed that male gender (OR 2.412; 95\%CI [1.212-4.797]) and chronic obstructive pulmonary disease (COPD) (OR 3.716; 95\%Cl [1.543-8.949]) were found to be independent predictors for the occurrence of major complications after primary RYGB.

\section{Conclusions}

This study showed a number of potential predictors, of which male gender and (COPD) after multivariate regression analysis were found to be independent predictive factors for the occurrence of major complications after primary RYGB. 


\section{INTRODUCTION}

For the treatment of morbid obesity, surgery has proven to be the most effective therapy as it results into long-term weight loss, an improved lifestyle and improvement of any obesity-related comorbidities. ${ }^{1}$ Nowadays, there are many surgical options, of which Roux-en-Y Gastric Bypass (RYGB), Sleeve Gastrectomy and Adjustable Gastric Banding are performed most frequently. ${ }^{2}$ Of these procedures, RYGB is currently still considered the gold standard. Even though the risk of serious postoperative complications following RYGB is relatively low, the high number of performed procedures results in a high absolute number of complications. ${ }^{3-5}$ Due to these large numbers, associated costs will certainly impact health care economy. Therefore, identifying potential predictors is important.

In 2011, the Michigan Bariatric Surgery Collaborative showed in a large retrospective that previous venous thromboembolism, mobility limitations, coronary artery disease, pulmonary disease, male gender, smoking history, age over 50 and procedure type are associated with an increased risk for the development of postoperative complications after bariatric surgery. ${ }^{3}$ Stenberg et al. have shown that age, additional operation, intraoperative adverse events and conversion to open surgery lead to a higher risk at serious postoperative complications. Furthermore, the complication risk was affected by the annual hospital volume to the prejudice of hospitals in a learning curve at the time of the operation. 4

Other studies report largely report on the same predictive factors, however results are sometimes contradictory. 3,4,6-10

Even though a number of studies have been published, this study aimed to identify potential risk factors for the development of major complications in the 30-day postoperative period after primary laparoscopic Roux-en-Y Gastric Bypass for further clarification of previous findings and possibly finding new predictive factors.

\section{METHODS AND DESIGN}

Since 2010, data on all patients undergoing bariatric surgery at the Catharina hospital, Eindhoven, the Netherlands, were collected prospectively in an online database. In this database, patient characteristics, operative details, complications and follow-up on weight loss and comorbidities were collected. For the current study, all patients undergoing primary Roux-en-Y gastric bypass (RYGB) between January 2010 and December 2013 were included. All early 
postoperative complications $<30$ days after primary RYGB were registered. Postoperative complications included surgical complications (anastomotic leakage, bleeding, incidental gastrointestinal perforation), infectious complications (intra-abdominal abscess, pneumonia, urinary tract infection, wound infection, gastroenteritis), major cardiovascular events, venous thromboembolism, anastomotic stenosis, marginal ulcer and postoperative ileus. All postoperative complications were classified according to ClavienDindo. ${ }^{11}$ Primary outcome of this study were all major complications. A complication was considered major when the Clavien-Dindo equaled 3a ( $\mathrm{A}$ complication requiring surgical, endoscopic or radiological intervention not under general anesthesia) or higher. All complications were diagnosed by the physician in charge at the time of the complication. The event was registered into the hospital's electronic system as well as the severity of the complication and later checked by two of the authors. The Clavien-Dindo depended on the treatment administered according to the hospital's electronic files.

Demographic and intra-operative variables included for analysis were age, gender, Body Mass Index (BMI), smoking, use of any alcohol, obesity-related comorbidities (diabetes mellitus type 2, hypertension, dyslipidemia, sleep apnea and osteo-articular disease), gastroesophageal reflux disease, chronic obstructive pulmonary disease, cardiovascular disease, previously performed upper gastrointestinal surgery, American Society of Anesthesiologists (ASA)classification, the use of a fast track protocol versus the conventional protocol, operative time and occurrence of intra-operative complications. Additionally, age $\geq 50$ years and $\mathrm{BMI} \geq 50 \mathrm{~kg} / \mathrm{m}^{2}$ were taken into account to separately analyze the older bariatric patient and the super-obese bariatric patient.

COPD is an obstructive lung disease including symptoms like shortness of breath, and coughing with the production of sputum and was considered when diagnosed by a general practitioner or a pulmonologist using a spirometry test. Cardiovascular disease was considered when the patient had a history myocardial infarction and/or underwent a percutaneous coronary intervention $(\mathrm{PCl})$.

\section{Treatment}

All morbidly obese patients attending the outpatient clinic were screened and discussed by a multidisciplinary team, consisting of dietitians, nursepractitioners, psychologists and surgeons. When necessary, patients underwent a preparatory course before bariatric surgery.

In earlier patients, follow-up was planned for one year after surgery, after which the patient was referred back to the general practitioner. Since the end of 2011, bariatric patients in this center undergo a structured five-year follow-up 
program with multiple visits to the outpatient clinic every year, including appointments with dietitians, nurses-practitioners, psychologists and bariatric surgeons.

\section{Procedure}

Patients were planned for surgery when they met the international guidelines for bariatric surgery as provided by the International Federation for the Surgery of Obesity and metabolic disorders (IFSO).

All patients planned for a laparoscopic primary Roux-en-Y gastric bypass were selected in the current study. Since 2011, patients are treated according to an implemented fast track protocol and by fixed operating room teams. ${ }^{12}$ Before then, patients received the conventional care. The surgery was performed by one of five expert bariatric surgeons, each performing well over fifty bariatric procedures per year.

Surgery was started by administering general anesthesia to the patient. The patient received thrombosis prophylaxis by 5000 units of low-molecular weight heparin. After adequate pre-oxygenation, induction is performed by the use of piritramide (0.2-0.3 mg/kg), Suxamethonium 1.0-1.5 $\mu \mathrm{g} / \mathrm{kg}$ and propofol $(2 \mathrm{mg} / \mathrm{kg})$ and the patient is intubated thereafter. During surgery, remifentanil is administered at a constant rate of $5-15 \mu \mathrm{g} / \mathrm{kg} /$ hour for analgesic maintenance. Anesthetic depth is maintained with desflurane (6.0\% vol.) in expired air. After completion of the anesthesia, the patient is placed in a reversed Trendelenburg position and the surgical procedure was initiated.

After insufflation of the abdomen using a Veress needle and the introduction of both the trocars and the camera, construction of the gastric bypass was initiated by creation of the gastric pouch using a stapler (EndoGIA; Covidien, New Haven, CT, USA). After completion of the pouch, the alimentary loop was pulled in an antecolic position, after which the antegastric end-to-side gastrojejunostomy was constructed by linear stapling (EndoGIA; Covidien, New Haven, CT, USA) and sutures using Polysorb ${ }^{T M}$ sutures until 2010 and V-Loc ${ }^{T M}$ (Covidien, Mansfield, MA, USA) since the beginning of 2011. The alimentary loop measured an estimated $150-180 \mathrm{~cm}$. The created biliopancreatic loop was estimated $60-80 \mathrm{~cm}$. The end-to-side jejunojejunostomy was created, also using a stapling device (EndoGIA; Covidien, New Haven, CT, USA) and sutures (Covidien, Mansfield, MA, USA). Closure of the mesenteric defects was not performed routinely. The procedure is finished by removal of the trocars under sight and closure of the skin. 


\section{Statistical analysis}

Data was managed and analyzed using SPSS version 22 (Windows; IBM Corporation, Armonk, NY. USA). Univariate associations between major complications and demographic covariates were analysed using Chi-square test and Fisher's exact test or independent samples $t$-test or Mann Whitney U-test. Covariates showing a trend towards significance for developing a major complication $(p<0.1)$ were considered for multivariable logistic regression analysis. Covariates displaying more than $1 \%$ missing values were excluded for multivariable regression analysis. A $p$-value $<0.05$ was considered significant. Quantitative data are denoted as mean +/- standard deviation (SD). Complications and evolution of comorbidities are presented as a percentage. Odds Ratio (OR) and 95\% confidence intervals $(\mathrm{Cl})$ were provided when applicable and considered significant when $\mathrm{OR}[95 \% \mathrm{Cl}] \neq 1$. Summative figures and tables were used when applicable.

No ethical approval was required for this study. Permission was granted by the Institutional Review Board for this retrospective study. All patients gave their informed consent for the prospective registration of their data in a large registry and the use for this study.

The described work has been carried out in accordance with the guidelines of the Declaration of Helsinki for experiments involving human subjects.

\section{RESULTS}

A total of 773 patients (85.5\% female) underwent primary laparoscopic Rouxen $Y$ gastric bypass. Mean age was $42.1 \pm 10.4$ years and the mean preoperative BMI was $42.8 \pm 4.3 \mathrm{~kg} / \mathrm{m}^{2}$. The mean highest recorded BMI was slightly higher at $44.4 \pm 4.7 \mathrm{~kg} / \mathrm{m}^{2}$. Obesity-related comorbidities were found in 423 patients (54.7\%).

Sixty-six patients (8.5\%) developed postoperative complications between day 0 and day 30 after surgery. Intra-operative complications occurred in nine patients $(1.2 \%)$. Conversion rate was $0.3 \%(n=2)$ and was performed in both cases due to incidental perforations of the small intestine.

There was no 30 -day mortality. The 90 -day mortality rate was $0.1 \%$; One patient died at day 70 day following RYGB due to an acute pulmonary hemorrhage after a complicated postoperative course due to a leakage of the jejunojejunostomy.

Major complications (Clavien-Dindo $\geq 3 a$ ) were observed in 55 out of 66 complications (7.1\%). Details of these events are shown in Table 9.1. 
Table 9.1 Major complications $<30$ days $(n=55)$.

\begin{tabular}{lc}
\hline & $\mathbf{n}$ (\% of total group, $\mathbf{n = 7 7 3 )}$ \\
\hline Anastomotic leakage & \\
$\quad$ Leakage gastrojejunostomy & $15(1.9 \%)$ \\
Leakage jejunojejunostomy & $2(0.3 \%)$ \\
Bleeding & $16(2.1 \%)$ \\
Gastrointestinal perforation & $4(0.5 \%)$ \\
Intra-abdominal abscess & $22(2.8 \%)$ \\
Pneumonia & $3(0.4 \%)$ \\
Wound infection & $2(0.3 \%)$ \\
Urinary tract infection & $2(0.3 \%)$ \\
Major cardiovascular event & $1(0.1 \%)$ \\
Anastomotic stenosis & $4(0.5 \%)$ \\
Marginal ulcer & $2(0.3 \%)$ \\
Gastro-enteritis & 0 \\
Postoperative ileus & 0 \\
Venous thromboembolism & 0 \\
\hline
\end{tabular}

Demographic and intra-operative parameters compared between patients with major complications and the patients with minor or no complications are shown in Table 9.2.

\section{Multivariate analysis}

A logistic regression analysis was performed using the factors (age, gender, BMI $\geq 50$, hypertension, COPD and previous upper gastrointestinal surgery) that were found to be significantly associated with occurrence of major complications in the univariate analysis (as is shown in Table 9.2). In this analysis, male gender (OR 2.412; 95\% Cl [1.212-4.797]) and COPD (OR 3.716; $95 \% \mathrm{Cl}$ [1.543-8.949]) were found to be independent predictors for the occurrence of major complications after primary RYGB, as is shown in Table 9.3. One-hundred and thirty two patients (17.1\%) were identified with one of the two factors. Only seven patients were found to have both independent predictive factors $(0.9 \%)$.

\section{Learning curve}

Learning curve may have been a confounder in the occurrence of major complications following RYGB. Therefore, the first cohort of 100 operated patients from the current study population were compared with the rest of the patients $(n=673)$.

Mean operative time $(83.2 \pm 27.6$ minutes vs $61.2 \pm 14.0$ minutes; $p<0.001)$ and mean hospital stay $(3.7 \pm 8.9$ days vs. $2.4 \pm 3.3$ days; $p<0.001)$ were significantly longer in the first 100 patients. The number of intraoperative complications 
(3.0\% vs. $0.9 \% ; p=0.099)$ and postoperative major complications (6.0\% vs. $7.3 \%$; $p=0.642$ ) did not differ significantly between groups.

Table 9.2 Patient characteristics $(n=773)$.

\begin{tabular}{|c|c|c|c|c|}
\hline & $\begin{array}{l}\text { MC }(n=55) \\
\text { Mean } \pm S D \\
n(\%)\end{array}$ & $\begin{array}{c}\text { No MC }(n=718) \\
\text { Mean } \pm \text { SD } \\
n(\%)\end{array}$ & $\begin{array}{c}p \text { - } \\
\text { value }\end{array}$ & $\begin{array}{l}\text { Missing } \\
\text { values } \\
\text { (n [\%]) }\end{array}$ \\
\hline Age (years) & $45.4 \pm 9.8$ & $41.8 \pm 10.4$ & 0.013 & 0 \\
\hline Age over $50(n)$ & $20(36.4 \%)$ & $176(24.5 \%)$ & 0.052 & 0 \\
\hline Gender (male : female) & 14: 41 & $98: 620$ & 0.017 & 0 \\
\hline Body Mass Index $\left(\mathrm{kg} / \mathrm{m}^{2}\right)$ & $43.3 \pm 4.7$ & $42.8 \pm 4.3$ & 0.397 & 0 \\
\hline BMI over $50 \mathrm{~kg} / \mathrm{m}^{2}(\mathrm{n})$ & 7 (12.7\%) & 47 (6.5\%) & 0.096 & 0 \\
\hline Smoking (n, \%) & $16(29.1 \%)$ & $148(20.6 \%)$ & 0.130 & $9(1.2 \%)$ \\
\hline History of smoking & $34(61.8 \%)$ & 305 (42.5\%) & 0.004 & $9(1.2 \%)$ \\
\hline Use of any alcohol (n, \%) & $14(25.5 \%)$ & $230(32.0 \%)$ & 0.479 & $23(3.0 \%)$ \\
\hline \multicolumn{5}{|l|}{ Pre-operative comorbidities (n, \%) } \\
\hline Type 2 Diabetes Mellitus & $18(32.7 \%)$ & $165(23.0 \%)$ & 0.101 & 0 \\
\hline Hypertension4 & $24(43.6 \%)$ & 235 (32.7\%) & 0.099 & 0 \\
\hline Dyslipidemia & $15(27.3 \%)$ & $137(19.1 \%)$ & 0.141 & 0 \\
\hline Sleep apnea & 7 (12.7\%) & 80 (11.1\%) & 0.720 & 0 \\
\hline Osteo-articular disease & $8(14.5 \%)$ & $113(15.7 \%)$ & 0.815 & 0 \\
\hline Gastroesophageal reflux disease (n, \%) & $22(40.0 \%)$ & $242(33.7 \%)$ & 0.343 & 0 \\
\hline Chronic obstructive pulmonary disease (n, \%) & $8(14.5 \%)$ & $26(3.6 \%)$ & 0.002 & 0 \\
\hline Cardiovascular disease $(\mathrm{n}, \%)$ & $3(5.5 \%)$ & $44(6.1 \%)$ & 1.000 & 0 \\
\hline Previous upper gastrointestinal surgery (n, \%) & $12(21.8 \%)$ & $98(13.6 \%)$ & 0.095 & 0 \\
\hline \multicolumn{5}{|l|}{ ASA $(n)$} \\
\hline I & $1(1.8 \%)$ & $21(2.9 \%)$ & 1.000 & 0 \\
\hline II & $47(85.5 \%)$ & $634(88.3 \%)$ & 0.530 & 0 \\
\hline III & 7 (12.7\%) & 63 (8.8\%) & 0.327 & 0 \\
\hline Conventional Care : Fast track Care (n) & $5: 50$ & $59: 659$ & 0.799 & 0 \\
\hline Operative time (min) & $68.6 \pm 27.7$ & $63.7 \pm 17.0$ & 0.461 & $1(0.1 \%)$ \\
\hline Intra-operative complication & $2(3.6 \%)$ & $7(1.0 \%)$ & 0.130 & 0 \\
\hline Polysorb suture : V-Loc suture & $5: 50$ & $59: 659$ & 0.799 & 0 \\
\hline
\end{tabular}

MC: major complication

Table 9.3 Results of logistic regression analysis ( $n=773)$.

\begin{tabular}{lccccc}
\hline & Beta (SE) & Significance & $\begin{array}{c}\text { Odds } \\
\text { ratio }\end{array}$ & \multicolumn{2}{c}{$\begin{array}{c}\text { 95\% Cl for Odds } \\
\text { Ratio }\end{array}$} \\
\cline { 4 - 6 } & & & & Lower & Upper \\
\hline Age & & & & & \\
Gender & $0.022(0.016)$ & 0.171 & 1.022 & 0.991 & 1.054 \\
Body Mass Index $\geq 50 \mathrm{~kg} / \mathrm{m}^{2}$ & $0.880(0.351)$ & $\mathbf{0 . 0 1 2}$ & 2.412 & 1.212 & 4.797 \\
Hypertension & $0.782(0.454)$ & 0.085 & 2.186 & 0.897 & 5.325 \\
Chronic obstructive pulmonary disease & $0.043(0.322)$ & 0.894 & 1.044 & 0.555 & 1.964 \\
Previous upper gastrointestinal surgery & $0.625(0.361)$ & 0.083 & 1.869 & 0.921 & 3.793 \\
Constant & $-3.985(0.677)$ & $<0.001$ & 0.019 & & \\
\hline
\end{tabular}

SE: standard error 


\section{DISCUSSION}

The aim of this study was to identify risk factors that may be predictive for the occurrence of major complications (Clavien-Dindo $\geq 3 a$ ) following RYGB. The international Clavien-Dindo classification for surgical complications was chosen to score the postoperative complications to create a better comparison tool for other studies. ${ }^{11}$ The main finding in this study was that male gender and COPD are significantly associated with the occurrence of major complications after primary RYGB.

The fact that male gender was found to be an independent risk factor for major complications confirms results of previous reports while keeping in mind that the scoring of the postoperative complications differed between these reports and the current study. ${ }^{7,8}$ Be that as it may, Stenberg et al. showed no significant predictive value for gender after primary RYGB for serious postoperative complications (Clavien-Dindo $\geq 3 b$ ). ${ }^{3,4,7}$

Previous findings also support the current result that COPD was identified to be an independent predictive factor for the occurrence of major complications after primary RYGB.(3) Be that as it may, the question remains to what extend this is caused by the actual COPD and what the involvement is of (history of) smoking, as it is one of the main risk factors for the development of COPD and is furthermore related to the occurrence of postoperative complications after RYGB. ${ }^{3,13}$ Besides that, assessment of collinearity between history of smoking and pulmonary diseases (including COPD) would have been important before including both parameters in the regression analysis. From a more practical point of view, it would be very interesting to see whether pre-operative cessation of smoking would decrease the risk of developing a major complication after surgery. Unfortunately, the current study was not designed to assess this hypothesis, so further research is necessitated.

In the current study population, postoperative complications were identified in only $8.5 \%$ of all patients, which is comparable with previous literature. ${ }^{3,4} \mathrm{~A}$ total of 55 major complications (7.1\%) were identified, of which anastomotic leakage, bleeding and intra-abdominal abscesses were the most common. The inhospital mortality rate $(0.1 \%)$ was comparable with previous literature. $3,4,6,9,14,15$

A number of demographic parameters were analyzed for their potential predictive value on the occurrence of major complications. In previous studies, age has shown to be an independent significant predictor for serious complications. ${ }^{3,4}$ The current study did not identify age as an independent predictive factor.

BMI was not found to be a risk factor for postoperative complications, however patients with a BMI $\geq 50 \mathrm{~kg} / \mathrm{m}^{2}$ showed a trend towards more major complications $(p=0.096)$ in comparison to patients with a lower BMI. Previous 
studies showed inconsistent results on the value of BMI towards postoperative complications. ${ }^{3,4,7}$

The obesity-related comorbidities (type 2 diabetes mellitus, hypertension, dyslipidemia, sleep apnea and osteo-articular disease) had no predictive value in the occurrence of major complications. Hypertension was identified as potential predictor, but appeared to have no independent predictive value according to the regression analysis $(p=0.894)$, however has been described before as risk factor for postoperative morbidity. ${ }^{7}$

The current study showed that patients who had previous upper gastrointestinal surgery show a trend towards more major complications. No previous studies were found on this matter. Data on the effect of previous upper gastrointestinal surgery (such as cholecystectomy) on the occurrence of (serious) postoperative complications after RYGB is scarce. Some studies show an increased need for adhesiolysis, potentially resulting in a prolonged operative time, conversion to open surgery, an increased incidence of wound infection and prolonged hospital stay. ${ }^{16,17}$ Further research is needed to delineate the effects of previous (upper abdominal) surgery on postoperative outcomes following RYGB.

The use of conventional care or fast track care was taken into account. Fast track care may be a protecting factor for the occurrence of postoperative complications as it aims to reduce the stress response and organ dysfunction and thereby shortening the time required for full recovery. ${ }^{18}$ This result was not reproduced by the current study. Furthermore, it remains the question whether fast track care may rather influence the speed of functional recovery and a faster return to work than the occurrence of postoperative complications.

Also other perioperative factors (operative time and intra-operative complications) showed no significant predictive value for the occurrence of major complications. Conversion to open procedure was not taken into account as a predictive factor as it was embedded in the intra-operative complications. The occurrence of postoperative or even major complications events may very well be surgeon or hospital dependent due to an inadequate level of experience. ${ }^{4,19-21}$ It seems unlikely that the occurrence of complications in this study population is dependent on an individual learning curve, as all procedures were performed by experienced bariatric surgeons, each performing at least 50-100 procedures every year. Additional analysis was performed to assess the hospital's experience, which did not influence the occurrence of intra-or postoperative complications.

This study, among others, is necessary to identify factors that may increase the risk of complications so proper strategies can be implemented to reduce future postoperative complications to a minimum. 
The most important limitation of the current study is relative small amount of patients. Even though a total of 773 patients were included for the current analysis, a larger number of patients is preferable in the search for predictors of major complications after RYGB, since the complication rate after RYGB is relatively low. ${ }^{3,420,21}$ Another limitation for the current study is the retrospective design, causing a limited registration of possible predictors. Coexisting conditions such as depression or hepatic conditions could not be scored due to insufficient registration.

In conclusion: This study showed a number of potential predictors, of which male gender and COPD after multivariate regression analysis were found to be independent predictive factors for the occurrence of major complications after primary RYGB. 


\section{REFERENCES}

1. Sjostrom L, Lindroos AK, Peltonen M, Torgerson J, Bouchard C, Carlsson B, et al. Lifestyle, diabetes, and cardiovascular risk factors 10 years after bariatric surgery. N Engl J Med. 2004; 351(26):2683-93.

2. Buchwald H, Oien DM. Metabolic/bariatric surgery worldwide 2011. Obes Surg. 2013;23(4): 427-36.

3. Finks JF, Kole KL, Yenumula PR, English WJ, Krause KR, Carlin AM, et al. Predicting risk for serious complications with bariatric surgery: results from the Michigan Bariatric Surgery Collaborative. Ann Surg. 2011;254(4):633-40.

4. Stenberg E, Szabo E, Agren G, Naslund E, Boman L, Bylund A, et al. Early complications after laparoscopic gastric bypass surgery: results from the Scandinavian Obesity Surgery Registry. Ann Surg. 2014;260(6):1040-7.

5. Weiner RA, El-Sayes IA, Theodoridou S, Weiner SR, Scheffel O. Early post-operative complications: incidence, management, and impact on length of hospital stay. A retrospective comparison between laparoscopic gastric bypass and sleeve gastrectomy. Obes Surg. 2013; 23(12):2004-12.

6. DeMaria EJ, Murr M, Byrne TK, Blackstone R, Grant JP, Budak A, et al. Validation of the obesity surgery mortality risk score in a multicenter study proves it stratifies mortality risk in patients undergoing gastric bypass for morbid obesity. Ann Surg. 2007;246(4):578-82; discussion 83-4.

7. Gupta PK, Franck C, Miller WJ, Gupta H, Forse RA. Development and validation of a bariatric surgery morbidity risk calculator using the prospective, multicenter NSQIP dataset. J Am Coll Surg. 2011;212(3):301-9.

8. Maciejewski ML, Winegar DA, Farley JF, Wolfe BM, DeMaria EJ. Risk stratification of serious adverse events after gastric bypass in the Bariatric Outcomes Longitudinal Database. Surg Obes Relat Dis. 2012;8(6):671-7.

9. Nguyen NT, Nguyen B, Smith B, Reavis KM, Elliott C, Hohmann S. Proposal for a bariatric mortality risk classification system for patients undergoing bariatric surgery. Surg Obes Relat Dis. 2013;9(2):239-46.

10. Sovik TT, Aasheim ET, Kristinsson J, Schou CF, Diep LM, Nesbakken A, et al. Establishing laparoscopic Roux-en-Y gastric bypass: perioperative outcome and characteristics of the learning curve. Obes Surg. 2009;19(2):158-65.

11. Dindo D, Demartines N, Clavien PA. Classification of surgical complications: a new proposal with evaluation in a cohort of 6336 patients and results of a survey. Ann Surg. 2004; 240(2):205-13.

12. Stepaniak PS, Heij C, Buise MP, Mannaerts GH, Smulders JF, Nienhuijs SW. Bariatric surgery with operating room teams that stayed fixed during the day: a multicenter study analyzing the effects on patient outcomes, teamwork and safety climate, and procedure duration. Anesth Analg. 2012;115(6):1384-92.

13. Mannino DM, Buist AS. Global burden of COPD: risk factors, prevalence, and future trends. Lancet. 2007;370(9589):765-73.

14. Benotti $P$, Wood GC, Winegar DA, Petrick AT, Still CD, Argyropoulos G, et al. Risk factors associated with mortality after Roux-en-Y gastric bypass surgery. Ann Surg. 2014;259(1): 123-30.

15. Morino M, Toppino M, Forestieri $P$, Angrisani L, Allaix ME, Scopinaro N. Mortality after bariatric surgery: analysis of 13,871 morbidly obese patients from a national registry. Ann Surg. 2007;246(6):1002-7; discussion 7-9.

16. Karayiannakis AJ, Polychronidis A, Perente S, Botaitis S, Simopoulos C. Laparoscopic cholecystectomy in patients with previous upper or lower abdominal surgery. Surg Endosc. 2004;18(1):97-101.

17. Wongworawat $M D$, Aitken $D R$, Robles $A E$, Garberoglio $C$. The impact of prior intra-abdominal surgery on laparoscopic cholecystectomy. Am Surg. 1994;60(10):763-6. 
18. Wilmore DW, Kehlet H. Management of patients in fast track surgery. BMJ. 2001;322(7284): 473-6.

19. Breaux JA, Kennedy $\mathrm{Cl}$, Richardson WS. Advanced laparoscopic skills decrease the learning curve for laparoscopic Roux-en-Y gastric bypass. Surg Endosc. 2007;21(6):985-8.

20. Schauer P, Ikramuddin S, Hamad G, Gourash W. The learning curve for laparoscopic Roux-en$Y$ gastric bypass is 100 cases. Surg Endosc. 2003;17(2):212-5.

21. Shikora SA, Kim JJ, Tarnoff ME, Raskin E, Shore R. Laparoscopic Roux-en-Y gastric bypass: results and learning curve of a high-volume academic program. Arch Surg. 2005;140(4):362-7. 



\section{CHAPTER}

10

Transection versus preservation of the neurovascular bundle of the lesser omentum in primary Roux-en-Y gastric bypass surgery

M.R. van Wezenbeek T.R. van Oudheusden J.F. Smulders

S.W. Nienhuijs M.D. Luyer

Surg Obes Relat Dis. 2016;12(2):283-9

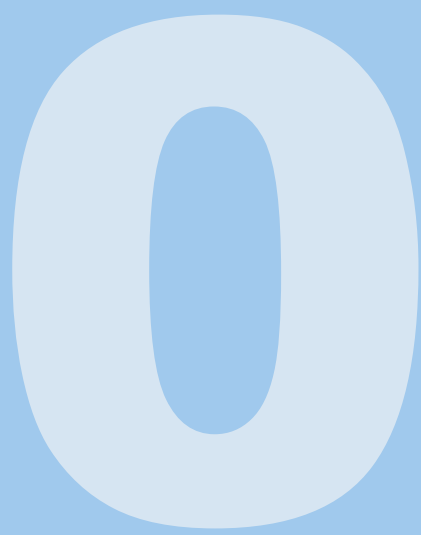




\section{ABSTRACT}

\section{Background \& Objectives}

A gastric pouch in Roux-en-Y gastric bypass (RYGB) surgery can be created after transection of the perigastric neurovascular bundle or by preserving these structures. Some surgeons choose to transect the neurovascular bundle (NBT), containing branches of the vagus nerve, since this might be related to additional weight loss, others advocate preservation (NBP) to reduce postoperative complications. This study assessed the effect of both techniques after primary RYGB.

\section{Setting \& Method}

All patients undergoing primary RYGB in a large bariatric center in the Netherlands between January 2010 and December 2013 were included. Patient demographics, operative details, postoperative complications and weight loss after one year were retrospectively analyzed.

\section{Results}

A total of 773 consecutive patients were included $(85.5 \%$ female). NBT was performed in 407 patients (52.7\%), whereas NBP was performed in 366 patients. There were no missing data and $81.2 \%$ of patients completed the 1 -year followup.

Postoperative complications were found in 66 patients (8.5\%). A total of 49 patients $(6.3 \%)$ either had an anastomotic leakage, postoperative bleeding or intra-abdominal abscess (NBT $8.8 \%$ vs. NBP 3.6\%, $p=0.003$ ). Percentage total body weight loss (NBT $34.5 \pm 6.9 \%$ vs. NBP 33.4 $\pm 6.9 ; p=0.011$ ) differed to a lesser extent between groups, although this was significant.

Neurovascular bundle transection was identified as independent factor amongst others for occurrence of leakage, bleeding and abscess development (OR 2.886; 95\%Cl [1.466-5.683]; $p=0.002$ ).

\section{Conclusions}

Transection of the neurovascular bundle in RYGB is associated with more complications. Furthermore, weight loss is not relevantly increased. Further research is necessitated to substantiate these findings. 


\section{INTRODUCTION}

Roux-en-Y gastric bypass (RYGB) is preferred by many for the treatment of morbid obesity, as it results in good long-term weight loss and an improvement in obesity-related comorbidities. ${ }^{1,2}$ Even though the postoperative complication rate is low after primary RYGB, the absolute number of complications still has a serious effect on health care costs. ${ }^{3,4}$ To create the pouch, the omental bursa has to be exposed for placement of the stapler. This can be done by transecting the neurovascular bundle containing branches of the vagus nerve and the left gastric artery and vein. ${ }^{5}$ Alternatively, this bundle can be preserved by creating an entry point via perigastric dissection.

There are some potential benefits of vagotomy or vagal nerve blocking in the treatment such as enhanced satiety, reduced food intake and additional weight loss. However, these studies are performed in patients with normal abdominal anatomy or rats. ${ }^{6-8}$ The first studies mentioned the potential benefits of vagotomy as a treatment for morbid obesity. ${ }^{7,9,10}$ However, more recent studies showed no difference in percentage weight loss between transection and preservation after RYGB in humans, whereas earlier, a postoperative difference in weight loss in favor of vagus nerve preservation was found in rats. ${ }^{11-14}$ No differences in postoperative complications were found. ${ }^{12-14}$ In terms of other effects lesser neurovascular bundle transection establishes a higher dumping symptomatology after RYGB compared to lesser omental preservation. ${ }^{12}$

It remains unclear how transection or preservation of the perigastric neurovascular bundle may affect the postoperative course and weight loss after RYGB-surgery. Therefore, the aim of this study is to assess the effect of neurovascular bundle transection (NBT) versus neurovascular bundle preservation (NBP) on the short-term course after RYGB.

\section{METHODS AND DESIGN}

All patients undergoing primary Roux-en- $Y$ gastric bypass surgery for the treatment of morbid obesity between January 2010 and December 2013 were included. A prospective database containing patient's characteristics, operative details, postoperative complications and follow-up, including weight loss and the evolution of any present comorbidities was used. One-year follow-up was considered for all patients. Long-term complications were not taken into account in the current study due to the short period of follow-up. All patients underwent follow-up after six weeks, six months and one year. For each followup, weight loss and evolution of any known comorbidities were registered. For 
the current study weight loss rates and the outcome of comorbidities after one year were included in the analysis.

Main outcome for this study was the occurrence of postoperative complications.

All complications were scored and additionally rated according to the ClavienDindo classification for surgical complications. ${ }^{15}$ A Clavien-Dindo score of $3 a$ or higher was considered a serious adverse event. As the occurrence of gastrojejunal anastomotic leakage, postoperative bleeding or intra-abdominal abscesses may directly be related to the type of technique used (transection or preservation of the neurovascular bundle). Transection of gastric vessels may lead to an increase of postoperative bleeding and potentially compromise the blood supply to the newly created gastrojejunal anastomosis in certain cases. Furthermore, abscess formation may also be reflected minor leakage of the gastrojejunal anastomosis or represent an infected hematoma.. Therefore, these complications were combined and considered the primary outcome for this study. Patients with more than one complication were counted as one complication to prevent overrepresentation.

The obesity surgery mortality risk score (OS-MRS) was included for preoperative assessment of the mortality risk. ${ }^{16}$

Postoperative weight loss was defined in three different ways: change in Body Mass Index (CBMI), percentage total body weight loss (\%TBWL) and percentage excessive weight loss (\%EWL). Total \%EWL was defined as a percentage of the amount of excess weight lost, in which excess was defined as the difference between the weight before surgery and the highest healthy weight, which is at a Body Mass Index (BMI) of $25 \mathrm{~kg} / \mathrm{m}^{2}{ }^{17}$

The evolution of the comorbidities was categorized into four different stages: stable, improved (reduced amount of medication used, improved lab results and/or a lower setting of a Continuous Positive Airway Pressure-device), resolved (no treatment and normalized lab results) and worse (opposite of improved). Any new comorbidities were registered, but were not taken into account in the analysis of the evolution of comorbidities.

\section{Treatment}

All patients underwent a preoperative screening at the outpatient clinic to assess whether or not they were qualified for a bariatric procedure. Every patient had to meet the standard IFSO guidelines for bariatric surgery. Patients were discussed in a multidisciplinary team consisting of a surgeon, a psychologist, and a dietitian.

Follow-up of the patients used to consist of one year guidance at the hospital by the bariatric team, containing a psychologist, a dietitian and a surgeon. After 
one year, follow-up was performed by a general practitioner. At the end of 2011, follow-up changed to a structured 5-year program with multiple appointments at the outpatient clinic with the surgeon, obesity nurse, dietitian, psychologist and physical therapist.

\section{Procedure}

All patients were planned for a RYGB by laparoscopic approach. Nowadays, patients are treated according to the fast track anesthesia protocol care, which was started in 2011. Before 2011, patients received the conventional anesthesia protocol care. Fast track care aims to reduce stress response and organ dysfunction after surgery and thereby greatly shorten the time required for full recovery. ${ }^{18,19}$

Surgery was performed by one of six different expert bariatric surgeons, each performing well over 50 bariatric procedures each year. The procedure started with creation of the gastric pouch using Endo GIA ${ }^{T M}$ staplers (Covidien, New Haven, CT, USA).

Neurovascular bundle transection (NBT) included transection of the lesser omentum using a linear stapler (Endo GIA ${ }^{T M}$ (Covidien, New Haven, CT, USA)). Preservation of the neurovascular bundle established by entering the omental bursa via perigastric dissection approximately $5-6 \mathrm{~cm}$ distal of the esophagogastric junction. The dissection was performed directly adjacent to the gastric wall until the omental bursa was entered, thereby taking care that the anterior and posterior nerves of Latarjet were preserved. ${ }^{20}$. The two different techniques were simultaneously used over the inclusion period. Whether the neurovascular bundle was transected or preserved depended on the preference of the operating surgeon.

After creation of the pouch, an end-to-side antecolic, antegastric gastrojejunostomy was constructed by linear stapling (EndoGIA ${ }^{\mathrm{TM}}$ (Covidien, New Haven, CT, USA)) and running suture. The alimentary loop measured an estimated 150-180 cm. The biliopancreatic loop was between 60 and $80 \mathrm{~cm}$. A jejuno-jejunostomy was created, also by linear stapling and running suture. Mostly, the procedure was finished by closing the mesenteric defect.

\section{Statistical analysis}

All data for the current study were collected prospectively and analyzed retrospectively. Management and analysis was performed using SPSS version 22 for Windows (IBM Corporation, Armonk, NY, USA). Quantitative data are denoted as mean +/- standard deviation (SD). Complications and evolution of comorbidities are presented as a percentage. 
Dependent on the type of variable, either student $t$-test or Mann Whitney Utest was used to determine any statistical significance between the observed differences among groups. A multivariate analysis was performed to identify potential predictors for the occurrence of either anastomotic leakage, postoperative bleeding or an intra-abdominal abscess. Differences were considered significant in case of a $p$ value less than 0.05 . Odds Ratio (OR) and 95\% confidence intervals $(\mathrm{Cl})$ were provided when applicable and considered significant when OR $(95 \% \mathrm{Cl}) \neq 1$. Summative figures and tables were used when applicable. Permission was granted by the Institutional Review Board for this retrospective study. Informed consent was obtained for prospective registration in a large registry by all patients.

\section{RESULTS}

A total of 773 patients ( $85.5 \%$ female) were included in the current study with a mean age of $42.1 \pm 10.4$ years and a mean $\mathrm{BMI}$ of $42.8 \pm 4.3 \mathrm{~kg} / \mathrm{m}^{2}$. In $407(52.7 \%)$ patients, the neurovascular bundle was transected (NBT) and in 366 (47.3\%) patients, the neurovascular bundle was preserved (NBP). Apart from gender (more men in the NBP group) and the distribution between conventional care and fast track care, patient characteristics did not differ significantly between the two groups, as shown in Table 10.1. All patients were planned for a laparoscopic approach. The conversion rate was $0.3 \%$.

In the postoperative 30-day period, a total of 66 patients developed complications (8.5\%). There was no 30-day mortality. Mortality rate after one year was $0.4 \%$. Three patients died during this period, of which one was RYGBrelated $(0.1 \%)$. This patient died during hospital admission after 70 days after an acute pulmonary hemorrhage. The postoperative course was initially complicated by leakage of the jejuno-jejunostomy. One patient died due to severe sepsis caused by a combination of pneumonia and a urinary tract infection, almost eight months after surgery. The third patient died from the consequences of metastasized vaginal carcinoma.

A total of 18 anastomotic leaks (2.3\%) occurred, requiring surgical reintervention in 15 cases (83.3\%). Hemorrhage occurred in 16 patients $(2.1 \%)$, of which 14 patients (87.5\%) required a surgical reintervention. In three patients, active bleeding was identified (two at the gastrojejunal anastomosis, one at the minor omentum, all after NBT). In 11 patients, only blood clots were found around the gastrojejunal anastomosis along with a significant postoperative decrease in hemoglobin levels of more than $2.0 \mathrm{mmol} / \mathrm{L}$. In the other two cases, active intraluminal bleeding at the site of the gastro- 
jejunostomy was managed with clipping via gastroscopy. Fifty-five out of 66 postoperative complications were considered to be a serious adverse event. Details on the perioperative and postoperative complications, Clavien-Dindo classification and weight loss after 1 year is shown in Table 2, stratified between NBT and NBP.

Table 10.1 Patient characteristics $(n=773)$.

\begin{tabular}{|c|c|c|c|}
\hline & $\begin{array}{c}\text { NBT } \\
n=407 \\
\text { Mean } \pm \text { SD }\end{array}$ & $\begin{array}{c}\text { NBP } \\
n=366 \\
\text { Mean } \pm \text { SD }\end{array}$ & $p$-value \\
\hline Age (years) & $42.0 \pm 10.5$ & $42.2 \pm 10.2$ & 0.803 \\
\hline Age $\geq 50$ (n, \%) & $103(25.3 \%)$ & $93(25.4 \%)$ & 0.974 \\
\hline Male : Female $(n)$ & $74: 333$ & $38: 328$ & 0.002 \\
\hline Body Mass Index $\left(\mathrm{kg} / \mathrm{m}^{2}\right)$ & $42.7 \pm 4.3$ & $42.9 \pm 4.4$ & 0.642 \\
\hline Body Mass Index $\geq 50(n, \%)$ & $27(6.6 \%)$ & 27 (7.4\%) & 0.686 \\
\hline Highest Body Mass Index $\left(\mathrm{kg} / \mathrm{m}^{2}\right)$ & $44.3 \pm 4.7$ & $44.5 \pm 4.8$ & 0.348 \\
\hline \multicolumn{4}{|l|}{ Pre-operative comorbidities (n, \%) } \\
\hline Diabetes Mellitus type 2 & $101(24.8 \%)$ & $82(22.4 \%)$ & 0.431 \\
\hline Hypertension & 137 (33.7\%) & 122 (33.3\%) & 0.923 \\
\hline Dyslipidemia & 79 (19.4\%) & 73 (19.9\%) & 0.852 \\
\hline Sleep apnea & $53(13.0 \%)$ & $34(9.2 \%)$ & 0.101 \\
\hline Osteo-articular disease & 71 (17.4\%) & $50(13.7 \%)$ & 0.148 \\
\hline Gastro-esophageal reflux (n, \%) & $133(32.7 \%)$ & $131(35.8 \%)$ & 0.362 \\
\hline History of smoking (n, \%) & $183(45.0 \%)$ & $156(42.6 \%)$ & 0.631 \\
\hline Previous upper gastrointestinal surgery (n, \%) & 60 (14.7\%) & $50(13.7 \%)$ & 0.668 \\
\hline \multicolumn{4}{|l|}{ ASA-classification (n, \%) } \\
\hline I & $12(2.9 \%)$ & $10(2.7 \%)$ & \\
\hline II & $361(88.7 \%)$ & $320(87.4 \%)$ & 0.774 \\
\hline III & 34 (8.4\%) & $36(9.8 \%)$ & \\
\hline Conventional Care vs. Fast track Care (n) & $23: 384$ & $41: 325$ & 0.005 \\
\hline
\end{tabular}

NBT: neurovascular bundle transection; NBP: neurovascular bundle preservation; ASA: American Society of Anesthesiologists

A total of 49 patients (6.3\%) either had gastrojejunal anastomotic leakage, postoperative bleeding or an intra-abdominal abscess, of which 36 patients (8.8\%) in the NBT group versus 13 patients (3.2\%) in the NBP group $(p=0.003)$. Six out of 16 patients (37.5\%) with gastrojejunal anastomotic leakage also developed an intra-abdominal abscess. One patient with postoperative bleeding developed an intra-abdominal abscess. No patient developed a gastrojejunal anastomotic leakage after a postoperative bleeding in this study. 
Table 10.2 Peri- and postoperative outcome $(n=773)$.

\begin{tabular}{|c|c|c|c|c|}
\hline & $\begin{array}{c}\text { NBT } \\
n=407 \\
\text { Mean } \pm \text { SD }\end{array}$ & $\begin{array}{c}\text { NBP } \\
n=366 \\
\text { Mean } \pm \text { SD }\end{array}$ & $\begin{array}{c}p- \\
\text { value }\end{array}$ & $\begin{array}{c}\text { Missing data } \\
\text { n (\%) }\end{array}$ \\
\hline Operative time (min) & $67.7 \pm 19.2$ & $60.0 \pm 15.5$ & 0.001 & $1(0.001 \%)$ \\
\hline Hospital stay (days) & $2.6 \pm 3.2$ & $2.5 \pm 5.5$ & $<0.001$ & 0 \\
\hline Intraoperative complications (n, \%) & $7(1.7 \%)$ & $2(0.5 \%)$ & 0.183 & 0 \\
\hline Postoperative complications (n, \%) & 44 (10.8\%) & $22(6.0 \%)$ & 0.017 & 0 \\
\hline Anastomotic leakage (n, \%) & $13(3.2 \%)$ & $5(1.4 \%)$ & 0.092 & 0 \\
\hline Leakage gastrojejunostomy (n, \%) & $11(2.7 \%)$ & $5(1.4 \%)$ & 0.193 & 0 \\
\hline Leakage enteroenterostomy (n, \%) & 2 (0.5\%) & 0 & 0.501 & 0 \\
\hline Bleeding $(n, \%)$ & $11(2.7 \%)$ & $5(1.4 \%)$ & 0.193 & 0 \\
\hline Gastro-intestinal perforation (n, \%) & $1(0.2 \%)$ & $3(0.8 \%)$ & 0.350 & 0 \\
\hline Intra-abdominal abscess (n, \%) & $18(4.4 \%)$ & $6(1.6 \%)$ & 0.026 & 0 \\
\hline Pneumonia (n, \%) & $4(1.0 \%)$ & $1(0.3 \%)$ & 0.377 & 0 \\
\hline Wound infection (n, \%) & $2(0.5 \%)$ & 0 & 0.501 & 0 \\
\hline Urinary tract infection $(\mathrm{n}, \%)$ & 0 & $2(0.5 \%)$ & 0.224 & 0 \\
\hline Gastro-enteritis (n, \%) & 0 & $1(0.3 \%)$ & 0.473 & 0 \\
\hline Major cardiovascular event (n, \%) & 0 & $2(0.5 \%)$ & 0.224 & 0 \\
\hline Anastomotic stenosis (n, \%) & $2(0.5 \%)$ & $2(0.5 \%)$ & 1.000 & 0 \\
\hline Marginal ulcer $(n, \%)$ & $4(1.0 \%)$ & $1(0.3 \%)$ & 0.377 & 0 \\
\hline Postoperative ileus (n, \%) & $1(0.2 \%)$ & 0 & 1.000 & 0 \\
\hline Readmission (n, \%) & $31(7.6 \%)$ & $13(3.6 \%)$ & 0.015 & 0 \\
\hline Clavien-Dindo 1 (n) & 0 & 1 & 0.473 & 0 \\
\hline Clavien-Dindo 2 (n) & 7 & 3 & 0.348 & 0 \\
\hline Clavien-Dindo 3a/3b (n) & 32 & 16 & 0.045 & 0 \\
\hline Clavien-Dindo 4a/4b (n) & 4 & 2 & 0.689 & 0 \\
\hline Clavien-Dindo 5 (n) & 1 & 0 & 1.000 & 0 \\
\hline Serious Adverse Events (Clavien-Dindo $\geq 3)(n, \%)$ & 37 (9.1\%) & $18(4.9 \%)$ & 0.024 & 0 \\
\hline Change in Body Mass Index $\left(\mathrm{kg} / \mathrm{m}^{2}\right)$ after 1 year & $14.8 \pm 3.4$ & $14.3 \pm 3.4$ & 0.010 & 145 (18.8\%) \\
\hline \% Total Body Weight Loss (\%TBWL) after 1 year & $34.5 \pm 6.9$ & $33.4 \pm 6.9$ & 0.011 & 145 (18.8\%) \\
\hline \% Excess Weight Loss (\%EWL) after 1 year & $85.7 \pm 19.5$ & $83.2 \pm 20.3$ & 0.123 & $145(18.8 \%)$ \\
\hline
\end{tabular}

NBT: neurovascular bundle transection; NBP: neurovascular bundle preservation.

\section{Comorbidities}

Improvement/resolution rates after one year for the entire study population for diabetes mellitus type 2, hypertension, dyslipidemia, sleep apnea and osteoarticular disease were 99.3\% (140/141), 88.7\% (189/213), 84.7\% (105/124), $97.0 \%(64 / 66)$ and $76.5 \%(78 / 102)$, respectively. The number of comorbidities differed from those at baseline, as 628 of 773 patients (81.2\%) completed the 1 -year follow-up. Comorbidities of the mentioned 628 patients were included for the 1-year improvement/resolution rates. 


\section{Operating surgeon}

Table 10.3 presents the number of procedures per year for each surgeon. Besides the number of RYGBs, each surgeon performed at least an equivalent amount of sleeve gastrectomies and a number of bariatric re-do procedures (data not shown). Differences in number of complications are assessed per year between the surgeons and also shown in Table 10.3.

The occurrence of either gastrojejunal anastomotic leakage, bleeding or intraabdominal abscess during the current study period was not different between the surgeons $(p=0.376)$.

Table 10.3 Complications and procedures per surgeon $(n=773)$.

\begin{tabular}{lccccccc}
\hline & A & B & C & D & E & F & $\boldsymbol{p}$-value \\
\hline 2010 & $1 / 41$ & $1 / 8$ & $1 / 15$ & 0 & 0 & 0 & 0.164 \\
2011 & $2 / 76$ & $0 / 31$ & $5 / 60$ & 0 & 0 & 5 & 0.295 \\
2012 & $3 / 63$ & $3 / 53$ & $12 / 69$ & $0 / 12$ & $2 / 37$ & 13 & 0.093 \\
2013 & $3 / 82$ & $1 / 25$ & $7 / 64$ & $4 / 50$ & $3 / 50$ & 19 & 0.473 \\
\hline NBT & 0 & 116 & 205 & 13 & 55 & 18 & \\
NBP & 261 & 1 & 1 & 50 & 34 & 19 & \\
\hline
\end{tabular}

Surgeon D: Started at end of 2012; Surgeon E: Started 2012; Surgeon F: Started at end of 2011, also performs bariatric procedures at another hospital; NBT: neurovascular bundle transection; NBP: neurovascular bundle preservation

\section{Multivariate analysis}

A univariate analysis (shown in Table 10.4) on the occurrence of anastomotic leakage, postoperative bleeding or intra-abdominal abscess, showed Age $(p=0.011)$, Age $\geq 50(p=0.026)$, presence of dyslipidemia $(p=0.006)$, a history of smoking $(p=0.009)$, neurovascular bundle transection $(p=0.003)$ and OS-MRS $(p=0.021)$ as potential predictor. OS-MRS, Dyslipidemia, History of smoking and neurovascular bundle transection were included in the multivariate analysis. Even though operating surgeon was not predictive for these complications $(p=0.376)$, this was also included in the analysis to further assess the influence of the operating surgeon on the occurrence of either gastrojejunal anastomotic leakage, bleeding or intra-abdominal abscess.

The multivariate analysis is shown in Table 10.5, which identified transection of the neurovascular bundle (OR 2.983; 95\% Cl $[1.442-6.171] ; p=0.003)$ and History of smoking (OR 2.115; $95 \% \mathrm{Cl}$ [1.147-3.903]; $p=0.016$ ) as independent predictive factors for occurrence of anastomotic leakage, or postoperative bleeding or intra-abdominal abscess after primary RYGB. 
Table 10.4 Univariate analysis $(n=773)$.

\begin{tabular}{|c|c|c|c|}
\hline & $\begin{array}{l}\text { Complications } \\
n=49 \\
\text { Mean } \pm \text { SD } \\
\end{array}$ & $\begin{array}{c}\text { No complications } \\
n=724 \\
\text { Mean } \pm \text { SD } \\
\end{array}$ & $p$-value \\
\hline Age (years) & $45.7 \pm 9.6$ & $41.9 \pm 10.4$ & 0.011 \\
\hline Age $\geq 50(n, \%)$ & $19(38.8 \%)$ & $177(24.4 \%)$ & 0.026 \\
\hline Male : Female $(n)$ & $10: 39$ & $102: 622$ & 0.224 \\
\hline Body Mass Index $\left(\mathrm{kg} / \mathrm{m}^{2}\right)$ & $43.1 \pm 5.0$ & $42.8 \pm 4.3$ & 0.626 \\
\hline Body Mass Index $\geq 50(n, \%)$ & $7(14.2 \%)$ & 47 (6.5\%) & 0.072 \\
\hline Highest Body Mass Index $\left(\mathrm{kg} / \mathrm{m}^{2}\right)$ & $44.2 \pm 4.9$ & $44.4 \pm 4.7$ & 0.637 \\
\hline \multicolumn{4}{|l|}{ Pre-operative comorbidities (n, \%) } \\
\hline Diabetes Mellitus type 2 & 17 (34.7\%) & $166(22.9 \%)$ & 0.061 \\
\hline Hypertension & 22 (44.9\%) & 237 (32.7\%) & 0.081 \\
\hline Dyslipidemia & 17 (34.7\%) & $135(18.6 \%)$ & 0.006 \\
\hline Sleep apnea & $5(10.2 \%)$ & $82(11.3 \%)$ & 0.810 \\
\hline Osteo-articular disease & $9(18.4 \%)$ & $112(15.5 \%)$ & 0.589 \\
\hline Gastro-esophageal reflux (n, \%) & 22 (44.9\%) & $242(33.4 \%)$ & 0.101 \\
\hline History of smoking (n, \%) & $30(61.2 \%)$ & $309(42.7 \%)$ & 0.009 \\
\hline History of thrombo-embolic events & 0 & $10(1.4 \%)$ & 1.000 \\
\hline Previous upper gastrointestinal surgery (n, \%) & $11(22.4 \%)$ & 99 (13.7\%) & 0.089 \\
\hline \multicolumn{4}{|l|}{ ASA-classification ( $n, \%)$} \\
\hline I & 1 & 21 & \\
\hline II & 41 & 640 & 0.376 \\
\hline III & 7 & 63 & \\
\hline Conventional Care vs Fast track Care (n) & $4: 45$ & $60: 664$ & 1.000 \\
\hline \multicolumn{4}{|l|}{ Obesity surgery mortality risk score (n) } \\
\hline Class A & 26 & 516 & \\
\hline Class B & 23 & 207 & 0.021 \\
\hline Class C & 0 & 1 & \\
\hline Neurovascular bundle transection vs preservation & $36: 13$ & $371: 353$ & 0.003 \\
\hline
\end{tabular}

Table 10.5 Results of logistic regression analysis $(n=773)$.

\begin{tabular}{lrrrrr}
\hline & Beta (SE) & Significance & $\begin{array}{c}\text { Odds } \\
\text { ratio }\end{array}$ & $\begin{array}{c}\mathbf{9 5 \%} \text { Cl for Odds } \\
\text { Ratio }\end{array}$ \\
\cline { 4 - 6 } & & & & Lower & Upper \\
\hline Obesity surgery mortality risk score & $0.523(0.331)$ & 0.114 & 1.687 & 0.881 & 3.229 \\
Dyslipidemia & $0.546(0.354)$ & 0.123 & 1.727 & 0.862 & 3.458 \\
Neurovascular bundle transection & $1.093(0.371)$ & $\mathbf{0 . 0 0 3}$ & 2.983 & 1.442 & 6.171 \\
Operating surgeon & $-0.059(0.120)$ & 0.624 & 0.943 & 0.746 & 1.192 \\
History of smoking & $0.749(0.312)$ & $\mathbf{0 . 0 1 6}$ & 2.115 & 1.147 & 3.903 \\
Constant & $-4.491(0.592)$ & $<0.001$ & 0.011 & & \\
\hline
\end{tabular}

$\mathrm{Cl}$ : Confidence Intervals; SE: Standard error.

\section{Post-hoc power analysis}

Due to the retrospective and exploratory nature of this study, a post-hoc power analysis was conducted to calculate the power in accordance to the primary outcome measure. Primary outcome was the incidence of either gastrojejunal 
anastomotic leakage, postoperative bleeding or intra-abdominal abscess. A type I error rate $(\alpha)$ was set at 0.05 . This calculation showed a statistical power of $90.8 \%$.

\section{DISCUSSION}

Until now, impact of neurovascular bundle transection or preservation in primary Roux-en-Y gastric bypass surgery was elusive. Some studies claim that transection is safe and efficient because it does not influence clinical, functional and laboratory results or even affect weight loss in an early stage and may technically alleviate gastric pouch formation., 84,21 Other studies show that vagus nerve preservation is associated with less postoperative dumping and reduced food intake. ${ }^{11,12}$ However, the impact on the early postoperative course was often unclear.

Current results showed that neurovascular bundle transection was an independent predictive factor for anastomotic leakage, postoperative bleeding or intra-abdominal abscess formation after RYGB. Additionally, a small significant difference was seen in \%TBWL and change in BMI after one year in favor of neurovascular bundle transection. A potential explanation may be the transection of both the left gastric artery and the left gastric vein. Hypothetically, transection of these vessels may compromise the blood supply to the newly created gastrojejunostomy, thereby increasing the risk of an anastomotic leakage. Furthermore, transection of these large blood vessels may hypothetically increase the chance at postoperative bleeding.

Male gender was significantly different between groups but did not influence the occurrence of anastomotic leakage, postoperative bleeding or intraabdominal abscess development. This is in contrast with previous studies showing that male gender is a predictor for postoperative complications after primary RYGB. ${ }^{3,4}$ Operative time was marginally increased in the NBT group. This may be either explained by the higher number of intra-operative complications, even though this was not significant, or it may reflect the fact that transection of the neurovascular bundle is a more time-consuming procedure than preservation of this anatomical structure. Furthermore, hospital stay was longer in the NBT group, most likely explained by the higher number of postoperative complications in this group.

Nonetheless, the exact mechanism underlying the observed differences in the postoperative complication rate between patients undergoing neurovascular bundle transection and patients in which the neurovascular bundle was preserved remains uncertain. 
When observing the results in Table 10.2, an increased occurrence of intraabdominal abscesses is observed in the NBT group. Besides the technical aspects that may lead to an increased abscess formation in patients undergoing neurovascular bundle transection, activity of the (abdominal) vagus nerve also is involved in regulating local and systemic inflammation. ${ }^{22,23}$ Interestingly, recently published data showed that the vagus nerve has an important regulatory role in inflammation during liver abscess formation in hamsters. Vagotomy disrupted the inflammatory response in this study and increased abscess formation in these hamster livers. ${ }^{24}$ To what extent this will be similar in the human body is unclear and further research is necessitated to substantiate these findings.

A possible confounding factor may have been the operating surgeon's experience. However, as all operating surgeons perform well over 50 bariatric procedures (including gastric bypasses, sleeve gastrectomies and bariatric redo procedures) every year in this large bariatric center, this seems unlikely. A univariate analysis showed that the operating surgeon was not predictive for the occurrence of postoperative complications in this study population $(p=0.376)$. Furthermore, Table 10.5 shows that in a multivariate analysis, operating surgeon is not predictive for the occurrence of gastrojejunal leakage, postoperative bleeding or intra-abdominal abscess.

The number of readmissions was higher in the NBT group, but can be explained by the higher number of postoperative complications in this group since not all complications occur during primary admission.

The rationale for transection of the neurovascular bundle is mainly based on the idea that it may provide some additional weight loss and that this technique may support a more straightforward method of gastric pouch creation. ${ }^{6,7,21,25,26}$ Both \%TBWL and CBMI were significantly higher in the NBT group. Since the groups showed a comparable BMI at baseline, this study supports the idea that vagotomy induces additional weight loss after primary RYGB. Be that as it may, the difference between the two groups, although significant, seems clinically irrelevant since the difference only involves one or two kilograms of additional weight loss after one year. Furthermore, the exact effect of limb lengths in this population remains unclear since the limb lengths differed between 150 and $180 \mathrm{~cm}$ and were estimated, not measured. This also applies for gastric pouch size.

There are some caveats to the current study design. First, this study is prone for bias due to the retrospective setup. Selection or randomization bias may have occurred, since the technique for gastric pouch creation depended on the operating surgeon. On the other hand, this is less likely since patients were not selected to be seen by a specific surgeon, but received an appointment with the first surgeon available. Secondly, due to the current study design, it remains 
unclear what factor of neurovascular bundle transection causes the increase in postoperative complications. A number of potential factors have been discussed, but further research is necessary to substantiate the actual impact of these factors.

\section{Conclusions}

Transection of the neurovascular bundle in RYGB is associated with more complications. Furthermore, weight loss is not relevantly increased. Further research is necessitated to substantiate these findings. 


\section{REFERENCES}

1. Sjostrom L. Bariatric surgery and reduction in morbidity and mortality: experiences from the SOS study. Int J Obes (Lond). 2008;32 Suppl 7:S93-7.

2. Sjostrom L, Lindroos AK, Peltonen M, Torgerson J, Bouchard C, Carlsson B, et al. Lifestyle, diabetes, and cardiovascular risk factors 10 years after bariatric surgery. $\mathrm{N}$ Engl J Med. 2004;351(26):2683-93.

3. Finks JF, Kole KL, Yenumula PR, English WJ, Krause KR, Carlin AM, et al. Predicting risk for serious complications with bariatric surgery: results from the Michigan Bariatric Surgery Collaborative. Ann Surg. 2011;254(4):633-40.

4. Stenberg E, Szabo E, Agren G, Naslund E, Boman L, Bylund A, et al. Early complications after laparoscopic gastric bypass surgery: results from the Scandinavian Obesity Surgery Registry. Ann Surg. 2014;260(6):1040-7.

5. Berthoud HR, Neuhuber WL. Functional and chemical anatomy of the afferent vagal system. Auton Neurosci. 2000;85(1-3):1-17.

6. Camilleri M, Toouli J, Herrera MF, Kulseng B, Kow L, Pantoja JP, et al. Intra-abdominal vagal blocking (VBLOC therapy): clinical results with a new implantable medical device. Surgery. 2008;143(6):723-31.

7. Kral JG. Vagotomy as a treatment for morbid obesity. Surg Clin North Am. 1979;59(6):1131-8.

8. Wang Y, Liu J. Combination of bypassing stomach and vagus dissection in high-fat dietinduced obese rats-a long-term investigation. Obes Surg. 2010;20(3):375-9.

9. Kral JG. Effects of truncal vagotomy on body weight and hyperinsulinemia in morbid obesity. Am J Clin Nutr. 1980;33(2 Suppl):416-9.

10. Kral JG, Gortz L. Truncal vagotomy in morbid obesity. Int J Obes. 1981;5(4):431-5.

11. Bueter $\mathrm{M}$, Lowenstein $\mathrm{C}$, Ashrafian $\mathrm{H}$, Hillebrand J, Bloom SR, Olbers $\mathrm{T}$, et al. Vagal sparing surgical technique but not stoma size affects body weight loss in rodent model of gastric bypass. Obes Surg. 2010;20(5):616-22.

12. Frantzides CT, Carlson MA, Shostrom VK, Roberts J, Stavropoulos G, Ayiomamitis G, et al. A survey of dumping symptomatology after gastric bypass with or without lesser omental transection. Obes Surg. 2011;21(2):186-93.

13. Okafor PN, Lien C, Bairdain S, Simonson DC, Halperin F, Vernon AH, et al. Effect of vagotomy during Roux-en-Y gastric bypass surgery on weight loss outcomes. Obes Res Clin Pract. 2015;9(3):274-80.

14. Perathoner A, Weiss $H$, Santner W, Brandacher G, Laimer $E$, Holler $E$, et al. Vagal nerve dissection during pouch formation in laparoscopic Roux-Y-gastric bypass for technical simplification: does it matter? Obes Surg. 2009;19(4):412-7.

15. Dindo D, Demartines N, Clavien PA. Classification of surgical complications: a new proposal with evaluation in a cohort of 6336 patients and results of a survey. Ann Surg. 2004;240(2): 205-13.

16. DeMaria EJ, Portenier D, Wolfe L. Obesity surgery mortality risk score: proposal for a clinically useful score to predict mortality risk in patients undergoing gastric bypass. Surg Obes Relat Dis. 2007;3(2):134-40.

17. Deitel $M$, Greenstein RJ. Recommendations for reporting weight loss. Obes Surg. 2003;13(2):159-60.

18. Geubbels N, Bruin SC, Acherman Yl, van de Laar AW, Hoen MB, de Brauw LM. Fast track care for gastric bypass patients decreases length of stay without increasing complications in an unselected patient cohort. Obes Surg. 2014;24(3):390-6.

19. Wilmore DW, Kehlet $\mathrm{H}$. Management of patients in fast track surgery. BMJ. 2001;322(7284): 473-6.

20. Skandalakis LJ, Gray SW, Skandalakis JE. The history and surgical anatomy of the vagus nerve. Surg Gynecol Obstet. 1986;162(1):75-85. 
21. Kral JG, Gortz L, Hermansson G, Wallin GS. Gastroplasty for obesity: long-term weight loss improved by vagotomy. World J Surg. 1993 Jan-Feb;17(1):75-8; discussion 9.

22. Borovikova LV, Ivanova S, Zhang M, Yang H, Botchkina GI, Watkins LR, et al. Vagus nerve stimulation attenuates the systemic inflammatory response to endotoxin. Nature. 2000;405(6785):458-62.

23. Maier SF, Goehler LE, Fleshner M, Watkins LR. The role of the vagus nerve in cytokine-to-brain communication. Ann N Y Acad Sci. 1998;840:289-300.

24. Sanchez-Aleman E, Quintanar-Stephano A, Escobedo G, Campos-Esparza Mdel R, CamposRodriguez R, Ventura-Juarez J. Vagotomy induces deregulation of the inflammatory response during the development of amoebic liver abscess in hamsters. Neuroimmunomodulation. 2015;22(3):166-80.

25. Sarr MG, Billington CJ, Brancatisano R, Brancatisano A, Toouli J, Kow L, et al. The EMPOWER study: randomized, prospective, double-blind, multicenter trial of vagal blockade to induce weight loss in morbid obesity. Obes Surg. 2012;22(11):1771-82.

26. Shikora S, Toouli J, Herrera MF, Kulseng B, Zulewski H, Brancatisano R, et al. Vagal blocking improves glycemic control and elevated blood pressure in obese subjects with type 2 diabetes mellitus. J Obes. 2013;2013:245683. 



\section{CHAPTER

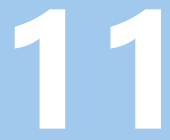

A specifically designed stent for anastomotic leaks after bariatric surgery: Experiences in a tertiary referral hospital

M.R. van Wezenbeek M.M. de Miliano

S.W. Nienhuijs

P. Friederich

L.P. Gilissen

Obesity Surgery. 2016;26(8):1875-80

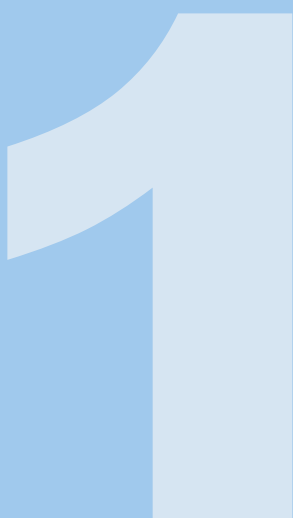




\section{ABSTRACT}

\section{Background}

Management of anastomotic leakage after either laparoscopic Roux-en-Y Gastric Bypass (LGBP) or laparoscopic Sleeve Gastrectomy (LSG) remains a burden. Various options are available for the treatment of these leaks. A newer and less invasive option for the treatment of leaks is the use of endoluminal stents. The main drawback for this treatment is stent migration. The current study describes the outcome of a new, specifically designed stent for the treatment of anastomotic leaks after bariatric surgery.

\section{Methods}

For this retrospective observational study, medical charts of patients undergoing bariatric surgery between October 1, 2010 and July 1, 2013 were reviewed. All patients with anastomotic leakage, treated with the bariatric Hanarostent, were included.

\section{Results}

Twelve patients were included out of a total of 1702 bariatric patients in the described period. Seven had a leakage after LSG, five after LGBP. An average of 2.4 endoscopic procedures and 1.25 stents were used per patient. Successful treatment was seen in nine out of 12 patients (75\%). Most common complication was dislocation or migration of the stent, occurring in eight patients (66.7\%).

\section{Conclusions}

The ECBB Hanarostent ${ }^{\circledR}$, which was specifically designed for post bariatric leakages, shows equal but not favorable success rates in this small series compared to previous reports on other types of stenting techniques. Despite the stent design, the complication rate is not reduced and the main future goal should be to target the high stent migration rate. 


\section{INTRODUCTION}

Obesity remains a global burden. For the treatment of morbid obesity, bariatric surgery has been the superior option for many years as it achieves good longterm weight loss, reduction of cardiovascular risk factors, improvement of obesity-related comorbidities and improved lifestyle. ${ }^{1-3}$

With an increasing number of procedures worldwide, it can be expected that the absolute number of complications after bariatric surgery will increase as well, even though these are relatively safe interventions. A recently published large study by Sanni et al. showed an average morbidity rate after laparoscopic gastric bypass (LGBP) of $5.1 \%$ and $1.4 \%$ after laparoscopic sleeve gastrectomy (LSG). ${ }^{4}$ The occurrence of anastomotic leakage, a major complication after either LGBP or LSG is, respectively, $1.6-4.8 \%$ and $1.7-2.4 \%$ as reported in more recent literature. ${ }^{5-8}$ Various options are available for the treatment of these leaks, such as conservative management (e.g. luminal suction via stomachpump, antibiotic treatment or external drainage), early surgical reintervention, fibrin glue treatment, over-the-scope-clipping and endoluminal stents. ${ }^{9-11}$ Until recently, surgical or radiological drainage, dismantling the anastomosis and resuturing of the leakage was the gold standard in case of anastomotic leakage. Be that as it may, performing surgery in a recently operated and infected area remains difficult and may contribute to the occurrence of complications. ${ }^{12} \mathrm{~A}$ less invasive method might be endoscopic stenting, which has its own disadvantages such as stent migration, obstruction, perforation or disintegration. ${ }^{13,14}$ Nevertheless, an increasing number of studies are reporting good results. ${ }^{15-20}$

More recently, repositionable and removable covered self-expandable metal stents (RReCoMSeS) have been developed, which appear feasible for temporary stenting of fistulae or leaks, however, results after bariatric surgery are still very limited. 13,14,18,21

This article describes the results in a series of bariatric patients with anastomotic leakage after either LGBP of LSG treated with the bariatric Hanaro ${ }^{\circledR}$ ECBB stent, a new repositionable and removable stent specifically designed for the treatment of anastomotic leaks after bariatric surgery.

\section{MATERIALS AND METHODS}

\section{Patients}

For this retrospective observational study, medical charts were reviewed of all patients undergoing bariatric surgery between October 1, 2010 and July 1, 
2013. All patients underwent treatment at the Catharina Hospital, Eindhoven, the Netherlands; a tertiary referral center for bariatric surgery.

All patients meeting the official IFSO guidelines for bariatric surgery underwent preoperative screening for eligibility by a multidisciplinary team, consisting of a dietician, a psychologist, a bariatric nurse and a surgeon. Those eligible for surgery were planned for either LGBP or LSG, based on the patients' screening data and their personal preference. All patients undergoing treatment with the new covered Hanaro ${ }^{\circledR}$ (M.I. Tech, Seoul, South Korea) ECBB bariatric stent for the treatment of either staple line leakage after sleeve gastrectomy or gastrojejunostomy leakage after gastric bypass surgery were included. The choice for stenting was made when other treatment options had not been successful in closure of the defect.

\section{Materials}

Bariatric surgery was performed by laparoscopic approach. The LSG has been described in detail before.(6) In case of LGBP, a standard Roux-en-Y gastric bypass was performed with an average biliopancreatic loop of $60-80 \mathrm{~cm}$ and an estimated alimentary loop of 150-180 cm. An antecolic and antegastric approach was used to create the gastrojejunostomy. All anastomoses were created by a combination of stapling (EndoGIA ${ }^{\circledR}$ Covidien, New Haven, CT, USA) and $\mathrm{V}-\mathrm{loc}^{\circledR}$ stitching (Covidien, New Haven, CT, USA).

In case of a suspected anastomotic leakage, the choice was made between additional imaging and a laparoscopic reintervention, mainly based on the clinical presentation of the patient (fever, tachycardia, elevated inflammation parameters, decreasing hemoglobin levels). Either a laparoscopic reintervention or radiological drainage was usually performed before endoscopic stenting was considered.

All patients undergoing endoscopic stent placement received monitored analgosedation using intravenous midazolam and/or fentanyl or propofol. The stent catheter was inserted over a guidewire in the upper gastrointestinal tract next to the endoscope and then released under direct endoscopic view. All patients were primarily treated with the Hanaro ${ }^{\circledR}$ ECBB bariatric stent with a length of $24 \mathrm{~cm}$ and a width of $30 \mathrm{~mm}$ (REF: ECBB-30-240-090). This is a long silicone covered nitinol stent, designed with a bottle-like shaped neck with a narrow lumen for the esophagus $(20 \mathrm{~mm})$, a shoulder to a wider lumen of $30 \mathrm{~mm}$ to bypass the wide gastric lumen and lassos at both ends to reposition or remove the stent (Figure 11.1). 


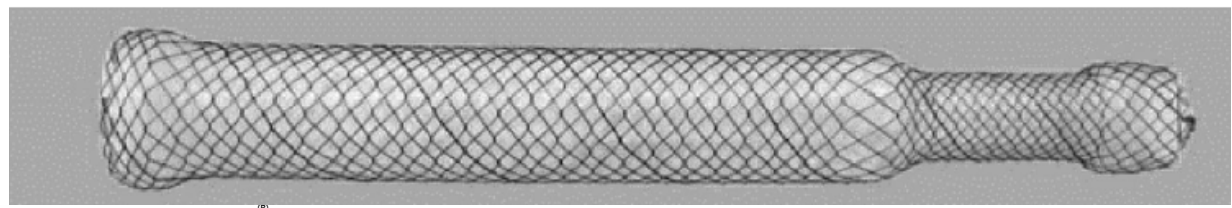

Figure 11.1 Hanaro ${ }^{\circledR}$ ECBB bariatric stent.

Primary outcome for this study was closure of the defect after stent removal. Secondary outcome were stent-related complications and fistula or stentrelated mortality, the number of endoscopies, the number of stents used, number of repositions and the differences in outcome between LSG and LGBP.

\section{Statistical analysis}

Data was collected retrospectively using Microsoft Excel 2010 for Windows. SPSS 22 for Windows was used for analysis of the data (SPSS Inc, Chicago, IL, USA).

Quantitative data are denoted as mean (range), whereas mortality rate and complications are presented as a percentage.

All procedures performed in studies involving human participants were in accordance with the ethical standards of the institutional research committee and with the 1964 Helsinki declaration and its later amendments or comparable ethical standards. Since this is a retrospective study, a formal consent is not required.

Informed consent was obtained from all individual participants included in the study.

\section{RESULTS}

Between October 2010 and July 2013, a total of 1702 bariatric procedures were performed. Among this number, a total of 826 sleeve gastrectomies were performed of which 21 (2.5\%) were a revisional procedure after earlier bariatric intervention, such as adjustable gastric banding (AGB). Furthermore, 874 gastric bypass procedures were performed, of which 264 (30.2\%) were a revisional procedure.

A total of 12 patients $(0.7 \%$ of all operated patients from that period) were treated with the ECBB bariatric type Hanarostent because of post-surgical leakage. Five were male and seven were female, with a mean age of 43.1 years (range 22-57). Further patient details are shown in Table 11.1. 
Table 11.1 Patient characteristics.

\begin{tabular}{|c|c|c|c|c|c|c|c|}
\hline $\begin{array}{l}\text { Patient } \\
\text { (No) }\end{array}$ & Gender & $\begin{array}{c}\text { Age } \\
\text { (years) }\end{array}$ & $\begin{array}{c}\text { BMI before } \\
\text { surgery } \\
\left(\mathrm{kg} / \mathrm{m}^{2}\right)\end{array}$ & Comorbidities $^{*}$ & ASA & Smoking & $\begin{array}{c}\text { Previous } \\
\text { procedure }\end{array}$ \\
\hline 1 & $M$ & 40 & 54.5 & $2,4,5$ & 3 & Yes & No \\
\hline 2 & $\mathrm{~F}$ & 48 & 43.7 & 1 & 2 & No & VBG \\
\hline 3 & M & 41 & 43.7 & 4 & 2 & Yes & No \\
\hline 4 & $\mathrm{~F}$ & 45 & 40.4 & 1,2 & 2 & No & No \\
\hline 5 & $\mathrm{~F}$ & 57 & 56.2 & $1,2,4$ & 2 & Yes & No \\
\hline 6 & M & 39 & 53.7 & None & 2 & No & No \\
\hline 7 & $\mathrm{~F}$ & 56 & 45.5 & 1,3 & 2 & No & No \\
\hline 8 & M & 22 & 42.5 & None & 2 & No & No \\
\hline 9 & $\mathrm{~F}$ & 43 & 41.8 & 1 & 2 & Yes & $A G B$ \\
\hline 10 & $\mathrm{~F}$ & 43 & 36.3 & 1, 3 & 2 & No & No \\
\hline 11 & M & 43 & 47.7 & $1,3,4,5$ & 2 & Yes & No \\
\hline 12 & $\mathrm{~F}$ & 40 & 57.9 & None & 2 & No & No \\
\hline $\begin{array}{l}\text { Mean } \\
\text { (range) }\end{array}$ & & $\begin{array}{c}43.1 \\
(22-57)\end{array}$ & $\begin{array}{c}46.99 \\
(36.3-57.9)\end{array}$ & & & & \\
\hline
\end{tabular}

*1: diabetes mellitus type 2; 2: hypertension; 3: dyslipidemia; 4: sleep apnea; 5: osteo-articular disease. BMI: body mass index; ASA: American Society of Anesthesiologists; VBG: vertical banded gastroplasty; AGB: adjustable gastric band

Seven patients had a leakage of the staple line after LSG and five patients had a leakage of the proximal anastomosis after LGBP (two as revisional procedure). The leak was diagnosed after a mean of 8.75 days after surgery (range 3-15) and the stent was placed after an average of 8 days (range 0-24) after diagnosis. All details on the outcome are shown in Table 11.2. All leaks in patients after LSG were found on the proximal side of the staple line, near the angle of His and the esophagogastric junction. Before stent insertion, most patients were treated with another, unsuccessful technique: five patients underwent a second laparoscopic intervention, seven patients underwent CTguided external drainage and one patient was first treated with over-thescope-clipping (OTS ${ }^{\circledast}$, Ovesco, Tübingen, Germany). Only one patient was immediately treated by means of a stent.

The Hanaro ${ }^{\circledR}$ bariatric stent was successful in closing the defect in nine out of 12 patients (75\%). A total of 29 stent-related endoscopic procedures were performed and a total of 15 stents were used, resulting in an average of 2.4 procedures per patient (range 1-3) and a mean number of 1.25 stents (range 1-2) used per patient. Successful treatment of the leak was seen in six out of seven LSG patients (85.7\%) and in three out of five LGBP patients $(60 \%)$. Stent-related complications were seen in nine out of 12 patients $(75 \%)$ involving ten stents (66.7\%). The most common complication was migration of the stent, occurring in eight out of 12 patients (66.7\%) including 9 out of 15 stents (60\%). 


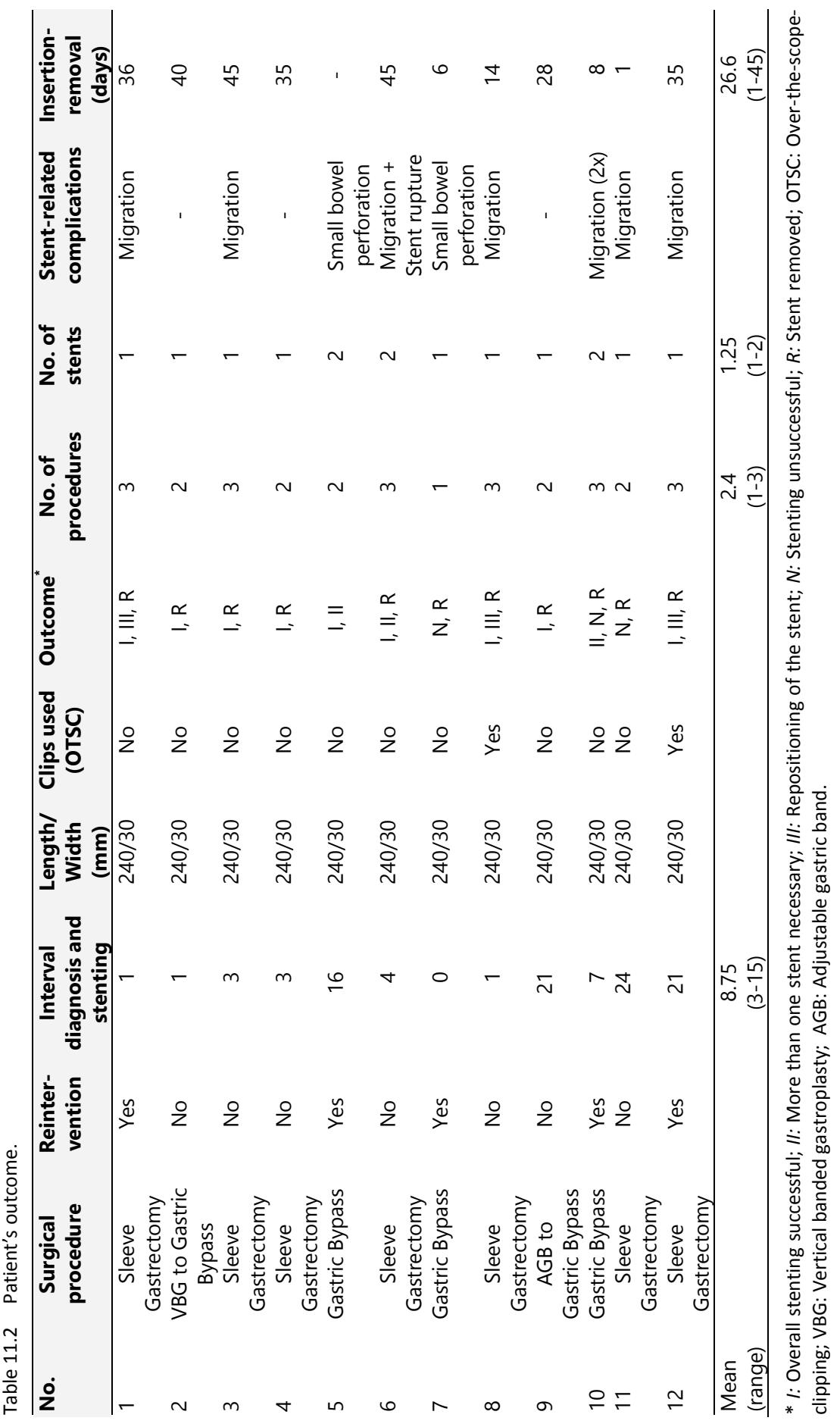


Three stents were repositioned, six stents were removed and one stent-in-stent technique was used to resolve the stent-related problem. Stent removal was performed by either endoscopy (five) or surgery (one). All migrated stents moved distally, in one case all the way to the terminal ileum. Mean time between placement and removal of a dislocated stent was 2.8 days (range 1-6 days). A small bowel perforation due to the stent was seen in two patients $(16.7 \%)$, in one case necessitating additional revisional surgery. LSG patients showed more stent-related complications than LGBP patients $85.7 \%$ vs $60 \%$ ). Dislocation of the stent was seen in six out of seven LSG patients (85.7\%) and in one out of five LGBP patients (20\%). Both small bowel perforations occurred in LGBP patients (40\%).

No leakage or stent-related mortality was seen in this study. One patient died six months after surgery due to severe septic shock caused by a pneumonia. Leakage was resolved (confirmed by gastroscopy), however the stent was not yet removed by the time the patient deceased and was taken into account as successful treatment in this analysis.

Stent removal in case of successful and uncomplicated closure of the defect was performed after a mean of 37.8 days (range 28-49).

Four stents showed a technical malfunction (26.7\%). Two stents unfolded only partially at the proximal side, one stent showed a 90-degree kinking and one stent was found to be partially ruptured.

\section{DISCUSSION}

Anastomotic leakage remains a serious and difficult to treat complication after bariatric surgery, with reported percentages of $1.6-4.8 \%{ }^{5-8}$ Even higher percentages are reported after revisional surgery (2.8-11.8\%). ${ }^{22-24}$ During the study period, the overall anastomotic leakage rate in this center was around $4 \%$, mostly after revisional procedures. With the development of modern endoscopic devices, such as the newly created Hanaro bariatric ECBB stent ${ }^{\circledR}$, new alternatives have risen for the treatment of leaks. However, clinical experience with these new devices is limited.

The current study population is too small to make a solid statement of the possible predictive value of the given baseline characteristics on the occurrence of leakage and the influence on the success rate of the Hanaro stent ${ }^{\circledR}$. The most important is the fact that these patients have to be compared to the general population undergoing bariatric surgery in the current bariatric center and that is not the goal of this manuscript. 
Multiple studies have described the effect of stenting for the treatment of leaks after bariatric surgery. The current success percentage of $75 \%$ is quite comparable to previously reported percentages between $78-94 \%{ }^{11,25-28}$ The current stent migration percentage of $58 \%$ is also similar to the upper limit of previously reported percentages $5-58 \% .{ }^{11,25-28}$

A trend to a higher rate of successful closing of the defect was seen after LSG compared with LGBP in this study as might be expected. Remarkably, LSG patients showed more stent-related complications (all being stent migration) as opposed to LGBP patients, an unexpected finding because the bariatric Hanarostent ${ }^{\oplus}$ was specifically designed for LSG associated leaks. ${ }^{29}$ No evident explanation was found for the high migration rate.

The main difference between previous studies and the current study, besides the design, appears to be the length and the width of the applied stent. Leenders et al. suggested that stent length may be a risk factor for stent migration, with a longer stent being related to a higher risk, possibly due to pronounced effect of peristaltic movements. ${ }^{21}$ The current report seems to confirm this. Furthermore, the current results show that the use of largediameter stents does not prevent stent migration in nonstenotic gastrointestinal tract segments. Many other solutions have been tried to reduce the stent migration rate in the past, such as a partially covered design, deployment of multiple stents, anti-migration struts and additional over-thescope clipping of the stent, but none posed as the definite solution for the prevention of migration. ${ }^{15,21,30,31}$ A partially covered ECBB stent may prevent migration, but stent removal is often more difficult. Stent migration is a problem for which a definite solution remains unclear and keeps necessitating more research.

The results in terms of number of endoscopic procedures, number of used stents and successful removal percentages are in accordance with, but not in favor of previously reported studies about endoscopic stenting of postsurgical fistulae or leaks, including the previously reported data of this center. $17,21,25,26,28$ Even though LGBP patients showed stent migration in only one out of five patients, two patients showed a small bowel perforation caused by the stent, a serious adverse event with possible severe clinical consequences.

Even though designed for leaks after sleeve gastrectomy, the ECBB Hanarostent ${ }^{\circledast}$ may be beneficial in the future for the treatment of leakage after Roux-en-Y gastric bypass. The reported bowel perforations may be caused by the stent's specific design. Length, diameter en material may have an influence, possibly by kinking of the stent and thereby applying additional pressure to the intestinal wall, increasing the chance of perforation.

This study contains a number of limitations. First of all, this study describes a relatively small series of 12 patients, but is currently the largest to report on the 
outcome of the bariatric ECBB Hanarostent ${ }^{\circledR}$. Larger, preferably prospective studies are needed. Another limitation is the heterogeneity of the group, since patients with leakage after either LSG or LGBP were included for analysis. Statistical analysis of LSG versus LGBP was not possible due to the small and heterogeneous group. A third limitation is that the stent was not the first treatment option of the leak in these patients, possibly influencing the outcome of the stent.

Furthermore, the first treatment varied between patients.

Nevertheless, this study shows the potential usefulness of the bariatric ECBB Hanarostent ${ }^{\circledR}$ in case of leakage after sleeve gastrectomy with the downfall of a high migration rate. No advantage is seen overall over other types of RReCoMSeS.

Furthermore, this study shows a potential benefit in the use of the bariatric Hanarostent ${ }^{\circledR}$ in the treatment of leakage of the gastrojejunostomy after LGBP, with the remark that the occurrence of bowel perforations has to be properly addressed first.

Improvements of the stent's design such as changes in length, diameter, shape and materials appear necessary and should lead to at least a lower migration rate of the stent. A biodegradable and (partially) covered stent, making stent removal unnecessary, may also be favorable.

In conclusion: The ECBB Hanarostent ${ }^{\circledR}$, which was specifically designed for post bariatric leakages, shows equal but not favorable success rates (75\%) in this small series compared to previous reports on other types of stenting techniques. Despite the stent design, the complication rate is not reduced and the main goal for the future should be to target the high migration rate $(58 \%)$ after stenting. 


\section{REFERENCES}

1. Ribaric G, Buchwald JN, McGlennon TW. Diabetes and weight in comparative studies of bariatric surgery vs conventional medical therapy: a systematic review and meta-analysis. Obes Surg. 2014;24(3):437-55.

2. Sjostrom L. Bariatric surgery and reduction in morbidity and mortality: experiences from the SOS study. Int J Obes. 2008;32 Suppl 7:S93-7.

3. Sjostrom L, Lindroos AK, Peltonen M, Torgerson J, Bouchard C, Carlsson B, et al. Lifestyle, diabetes, and cardiovascular risk factors 10 years after bariatric surgery. N Engl J Med. 2004; 351(26):2683-93.

4. Sanni A, Perez S, Medbery R, Urrego HD, McCready C, Toro JP, et al. Postoperative complications in bariatric surgery using age and BMI stratification: a study using ACS-NSQIP data. Surg Endosc. 2014;28(12):3302-9.

5. Aurora AR, Khaitan L, Saber AA. Sleeve gastrectomy and the risk of leak: a systematic analysis of 4,888 patients. Surg Endosc. 2012;26(6):1509-15.

6. van Rutte PW, Smulders JF, de Zoete JP, Nienhuijs SW. Outcome of sleeve gastrectomy as a primary bariatric procedure. Br J Surg. 2014;101(6):661-8.

7. Weiner RA, El-Sayes IA, Theodoridou S, Weiner SR, Scheffel O. Early post-operative complications: incidence, management, and impact on length of hospital stay. A retrospective comparison between laparoscopic gastric bypass and sleeve gastrectomy. Obes Surg. 2013;23(12):2004-12.

8. Whitlock KA, Gill RS, Ali T, Shi X, Birch DW, Karmali S. Early Outcomes of Roux-en-Y Gastric Bypass in a Publically Funded Obesity Program. ISRN Obesity. 2013;2013:296597.

9. Jacobsen HJ, Nergard BJ, Leifsson BG, Frederiksen SG, Agajahni E, Ekelund $M$, et al. Management of suspected anastomotic leak after bariatric laparoscopic Roux-en-y gastric bypass. Br J Surg. 2014;101(4):417-23.

10. Schiesser M, Kressig P, Bueter M, Nocito A, Bauerfeind P, Gubler C. Successful endoscopic management of gastrointestinal leakages after laparoscopic Roux-en-Y gastric bypass surgery. Dig Surg. 2014;31(1):67-70.

11. Simon F, Siciliano I, Gillet A, Castel B, Coffin B, Msika S. Gastric leak after laparoscopic sleeve gastrectomy: early covered self-expandable stent reduces healing time. Obes Surg. 2013;23(5): 687-92.

12. Urschel JD. Esophagogastrostomy anastomotic leaks complicating esophagectomy: a review. Am J Surg. 1995;169(6):634-40.

13. van Boeckel PG, Sijbring A, Vleggaar FP, Siersema PD. Systematic review: temporary stent placement for benign rupture or anastomotic leak of the oesophagus. Aliment Pharmacol Ther. 2011;33(12):1292-301.

14. Salminen $P$, Gullichsen $R$, Laine $S$. Use of self-expandable metal stents for the treatment of esophageal perforations and anastomotic leaks. Surg Endosc. 2009;23(7):1526-30.

15. Puig CA, Waked TM, Baron TH, Sr., Wong Kee Song LM, Gutierrez J, Sarr MG. The role of endoscopic stents in the management of chronic anastomotic and staple line leaks and chronic strictures after bariatric surgery. Surg Obes Relat Dis. 2014;10(4):613-7.

16. Victorzon M, Victorzon S, Peromaa-Haavisto P. Fibrin glue and stents in the treatment of gastrojejunal leaks after laparoscopic gastric bypass: a case series and review of the literature. Obes Surg. 2013;23(10):1692-7.

17. Donatelli G, Dhumane P, Perretta S, Dallemagne B, Vix M, Mutter D, et al. Endoscopic placement of fully covered self expanding metal stents for management of post-operative foregut leaks. J Minim Access Surg. 2012;8(4):118-24.

18. Swinnen J, Eisendrath P, Rigaux J, Kahegeshe L, Lemmers A, Le Moine O, et al. Self-expandable metal stents for the treatment of benign upper Gl leaks and perforations. Gastrointest Endosc. 2011;73(5):890-9. 
19. Sharaiha RZ, Kim KJ, Singh VK, Lennon AM, Amateau SK, Shin EJ, et al. Endoscopic stenting for benign upper gastrointestinal strictures and leaks. Surg Endosc. 2014;28(1):178-84.

20. Choi HJ, Lee BI, Kim JJ, Kim JH, Song JY, Ji JS, et al. The temporary placement of covered selfexpandable metal stents to seal various gastrointestinal leaks after surgery. Gut Liver. 2013;7(1):112-5.

21. Leenders BJ, Stronkhorst A, Smulders FJ, Nieuwenhuijzen GA, Gilissen LP. Removable and repositionable covered metal self-expandable stents for leaks after upper gastrointestinal surgery: experiences in a tertiary referral hospital. Surg Endosc. 2013;27(8):2751-9.

22. Coblijn UK, Verveld CJ, van Wagensveld BA, Lagarde SM. Laparoscopic Roux-en-Y gastric bypass or laparoscopic sleeve gastrectomy as revisional procedure after adjustable gastric band--a systematic review. Obes Surg. 2013;23(11):1899-914.

23. Perathoner A, Zitt M, Lanthaler M, Pratschke J, Biebl M, Mittermair R. Long-term follow-up evaluation of revisional gastric bypass after failed adjustable gastric banding. Surg Endosc. 2013;27(11):4305-12.

24. Zhang L, Tan WH, Chang R, Eagon JC. Perioperative risk and complications of revisional bariatric surgery compared to primary Roux-en-Y gastric bypass. Surg Endosc. 2014.

25. Eisendrath P, Cremer M, Himpens J, Cadiere GB, Le Moine O, Deviere J. Endotherapy including temporary stenting of fistulas of the upper gastrointestinal tract after laparoscopic bariatric surgery. Endoscopy. 2007;39(7):625-30.

26. Eubanks S, Edwards CA, Fearing NM, Ramaswamy A, de la Torre RA, Thaler KJ, et al. Use of endoscopic stents to treat anastomotic complications after bariatric surgery. J Am Coll Surg. 2008;206(5):935-8; discussion 8-9.

27. Puli SR, Spofford IS, Thompson CC. Use of self-expandable stents in the treatment of bariatric surgery leaks: a systematic review and meta-analysis. Gastrointest Endosc. 2012;75(2):287-93.

28. Salinas A, Baptista A, Santiago E, Antor M, Salinas H. Self-expandable metal stents to treat gastric leaks. Surg Obes Relat Dis. 2006;2(5):570-2.

29. Oshiro T, Kasama K, Umezawa A, Kanehira E, Kurokawa Y. Successful management of refractory staple line leakage at the esophagogastric junction after a sleeve gastrectomy using the HANAROSTENT. Obes Surg. 2010;20(4):530-4.

30. Blackmon SH, Santora R, Schwarz P, Barroso A, Dunkin BJ. Utility of removable esophageal covered self-expanding metal stents for leak and fistula management. Ann Thorac Surg. 2010; 89(3):931-6; discussion 6-7.

31. Uitdehaag MJ, van Hooft JE, Verschuur EM, Repici A, Steyerberg EW, Fockens $P$, et al. A fullycovered stent (Alimaxx-E) for the palliation of malignant dysphagia: a prospective follow-up study. Gastrointest Endosc. 2009;70(6):1082-9. 


\section{CHAPTER 12}

Summary, discussion \& future perspectives
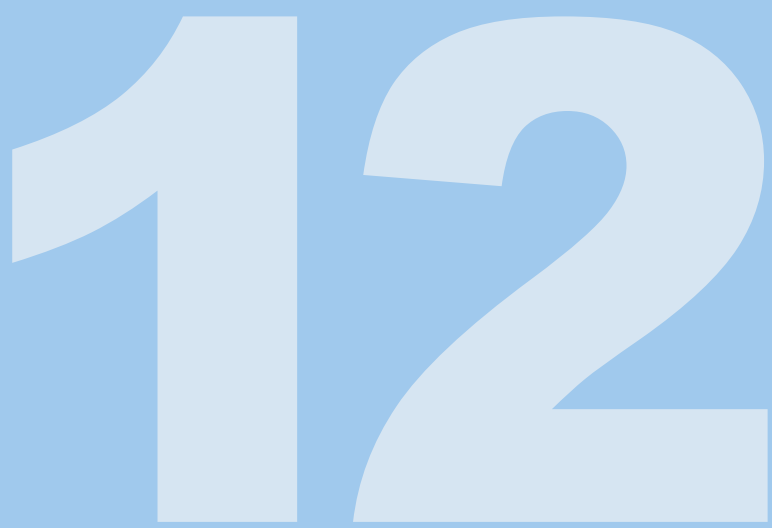



\section{SUMMARY \& DISCUSSION}

As stated in the introduction, bariatric surgery is the best therapeutic option for the treatment of morbid obesity in terms of weight loss and a reduction of obesity-related comorbidities, while reducing the lifetime health care costs and elevating the postoperative quality of life. ${ }^{1-4}$

Although the majority of bariatric patients benefit from these positive outcomes, a small but significant amount of patients is confronted with some type of complication. The aim of this thesis was to contribute to the reduction of before mentioned complications by analyzing current results of revisional surgery, finding predictors for complications and assessing the effect of different intra-operative techniques.

\section{Part one}

To develop a notion on treating complications in the future, it is essential to have a proper understanding of the past. Therefore, Chapter 1 described the long-term outcome of primary vertical banded gastroplasty (VBG) in 392 patients. With a mean follow-up of 66 months, VBG provided an acceptable mean excess weight loss of $53 \%$, with a $55 \%$ improvement of obesity-related comorbidities. Nonetheless, this chapter underlined the major disadvantage of this procedure, with a reported complaint rate of $58 \%$ and a total revision rate of nearly $40 \%$.These numbers are similar to previously reported statistics and strengthen the believe to abandon this old restrictive bariatric procedure. ${ }^{5-9}$ Nowadays, sleeve gastrectomy appears more suitable, as the first reported results are superior to those of the VBG. ${ }^{10}$

Even though VBG is abandoned in the Netherlands, an interest should be taken in its anatomical similarities with the more modern banded gastric bypass. The main difference between the two procedures is the presence or absence of the pyloric valve. The results of the older VBG may be helpful in improving the banded gastric bypass, as literature is already reporting a complication rate up to $20 \%{ }^{11}$

It is becoming more and more clear that the success of bariatric also depends on many psychological factors such as eating behavior. ${ }^{12}$ Chapter 2 attempted to find more of these predictors for success or failure. Among others, it identified that successful patients depend less on the procedure than patients who failed. This supports the results presented by Mitchell et al. that proper lifestyle changes may be more important than the bariatric procedure itself for achieving sufficient long-term weight loss. ${ }^{13}$ This chapter showed a few other potential predictors. Unfortunately, the outcome of this study does not provide 
the solution for a standard pre- and postoperative psychological evaluation. On the contrary, psychological guidance before and after bariatric surgery needs to be individualized, while keeping track of the major (psychosocial) risk factors associated with failure. Unfortunately, the conclusion remains similar to the majority of other psychological bariatric publications: more (high quality) research is highly needed and current results are inconsistent. ${ }^{13-17}$

After developing an understanding of the primary VBG, the next task was understanding its revisional procedures. Chapter 3 described the results of different revisional procedures after primary failed VBG. Even though the groups in this study were skewed and some of the groups were small, the results were still very clear. The best revisional option after failed VBG is a Rouxen-Y gastric bypass (RYGB) when compared to revision of the VBG or conversion to a sleeve gastrectomy. This study proved a superiority for conversion to RYGB in terms of (additional) weight loss, improvement of obesity-related comorbidities, the occurrence of long-term complaints or complications and the need for additional revisional surgery.

Revision of the VBG or conversion to sleeve gastrectomy should be not be considered as revisional option for failed primary VBG based on these results. These statements are supported by other studies on this subject. ${ }^{9,18-20}$ Therefore, if revision of a failed primary VBG is indicated, it is suggested to only perform a revision to RYGB unless this is technically not possible.

As shown in Chapter 3, RYGB appears to be a feasible and effective option as revisional procedure after VBG. Can this statement be extended to other restrictive procedures such as the adjustable gastric banding (AGB)? The suggestion is supported by previously published literature, which shows good results of RYGB as revision after failed $A G B$. Furthermore, conversion to RYGB appears to be superior after failed $A G B$ when compared to band revision or conversion to sleeve gastrectomy. ${ }^{21-24} \mathrm{~A}$ drawback is a high postoperative complication rate after revision, as a gastric pouch is created around the old location of the AGB. ${ }^{24,25}$

Chapter 4 hypothesized that the complication rate may be lower when converting a failed sleeve gastrectomy to RYGB. The thought behind this hypothesis was to further strengthen the believe that $A G B$ should be abandoned as a primary bariatric procedure, besides the high long-term failure rate of this procedure. ${ }^{23,26,27}$ Furthermore, this study investigated the effectiveness of RYGB as revisional procedure after either failed AGB or sleeve gastrectomy (SG).

The results did not support the believe that conversion to RYGB after sleeve is a safer procedure when compared to RYGB after failed $A G B$ as it reported a 
similar postoperative complication rate ( $8.8 \%$ vs. $11.8 \% ; p=0.530)$. Although these results suggested a similar effectiveness of RYGB as revisional procedure after either AGB or SG, they should be interpreted with caution. During followup, a slight decrease in total weight loss was observed in the SG group compared to an increase in the AGB group. The results in this chapter confirmed that RYGB is a valuable option as revisional procedure after failed $A G B$, however, it cannot be irrefutably stated that it is as valuable after failed sleeve gastrectomy for achieving additional weight loss.

Keeping the high failure rate of the AGB and the high complication rate after revision in mind, $A G B$ should not be performed routinely as a primary bariatric procedure until reliable selection criteria are developed. ${ }^{23,26-28}$ Nowadays, both SG and RYGB are considered longer lasting and safer bariatric procedures. ${ }^{29-31}$

Recently, fast track protocols were introduced to counterattack the increasing demand for bariatric surgery and have proven to be safe and effective..$^{32,33}$ It is expected that many revisional procedures will be performed over the next years when considering the amount of performed VBG's, AGB's and SG's in the last decades. With the increasing demand for revisional surgery, the question rises whether fast track surgery would be safe in this challenging group of patients. The main goal would be to stabilize or even decrease the significant complication rate. Despite being comparable with previous literature, Chapter 3 and 4 reported a high complication rate between $8-13 \% .{ }^{34-38}$

Chapter 5 reported on the results of the use of a fast track protocol in revisional bariatric surgery. This study reported a significant decrease of the postoperative complication rate after implementing the fast track protocol. Based on these results, fast track care is safe to implement in bariatric revisional surgery, potentially reducing health care costs by lowering hospital stay and increasing logistics without increasing the complication rate. ${ }^{32,33}$

Even though a significantly lower complication rate is reported $(19.2 \%$ vs. $11.2 \% ; p=0.038$ ), caution should be taken in assuming that fast track care decreases the complication rate. Strong confounding factors such as an increased experience of the operating team, the surgeon's learning curve or improved surgical equipment may also be the cause of the reduced complication rate over the years. ${ }^{39,40}$

When aiming for relief of reflux complaints, RYGB appears to be effective, but the value of RYGB as revision after failed sleeve gastrectomy to achieve additional weight loss, is debatable. ${ }^{41}$ Casillas et al. reported similar results as shown in Chapter 4, which is a decline in (additional) weight loss over time after RYGB as revisional for failed primary sleeve gastrectomy. ${ }^{42}$ Yorke et al. reported on the lowest $\mathrm{BMI}$ after revision of primary sleeve gastrectomy to RYGB. A 
mean BMI of $36.4 \mathrm{~kg} / \mathrm{m}^{2}$ is reported. Despite the fact that this is lower than the reported $\mathrm{BMI}$ after primary sleeve gastrectomy, this is well within range to be called morbid obesity. ${ }^{43}$ Even though some studies do report good results on RYGB as revisional procedure after failed primary SG, the search for a potentially better alternative has started.

Homan et al. and Carmeli et al. have explored the potentials of the biliopancreatic diversion compared with Roux-en-Y gastric bypass. Even though a higher weight loss is reported, a drawback is that this type of procedure is technically more challenging and may lead to an even higher rate of postoperative complications. ${ }^{44,45}$ Therefore, the single-anastomosis duodenoileal bypass (SADI) was chosen to investigate as an alternative for RYGB after sleeve gastrectomy in Chapter 6. Even though the knowledge on this relatively new procedure is limited, the first results seem promising. ${ }^{46,47}$

After 12 months, this study showed a slightly higher weight loss and less early postoperative complications in the SADI group, which were not significantly different when compared to the RYGB group. Biggest drawbacks were the limited group sizes and the total follow-up. Based on these limited results, SADI appears to be safer compared to a biliopancreatic diversion in terms of postoperative complications.

Recent studies do support the hypothesis that SADI is a valuable alternative to achieve additional weight loss after sleeve gastrectomy. ${ }^{48-50}$ The biggest drawback of these results are the retrospective nature of the studies. Good prospective, preferably randomized controlled trials are necessary to place current results into perspective. With the current knowledge of the SADI and the results from Chapter 6 in mind, Chapter 7 reported on a study protocol for a prospective, randomized controlled trial.

\section{Part two}

As mentioned before, part two of this thesis focused on postoperative complications after bariatric surgery. Obviously, prevention is the best way to lower the postoperative complication rate. Prevention is based on knowing the risk factors. Chapter 8 reported on predicting factors for the occurrence of major complications (Clavien-Dindo $\geq 3$ a) after primary RYGB. Even though the study population was small (only 773 patients), this study identified two independent risk factors: male gender and chronic obstructive pulmonary disease.

It seems unlikely that the male gender itself is a risk factor for the occurrence of postoperative complications, however, the male bariatric population are more often diagnosed with metabolic syndrome. This in turn is correlated to an 
increased risk at postoperative complications in bariatric surgery. ${ }^{51}$ Is it an option to stabilize or even treat this syndrome before performing bariatric surgery so the postoperative complication rate may be lowered? Recent literature reports on the interactions between gut microbiota, host genetics and the development of metabolic syndrome and even obesity. ${ }^{52,53}$ This knowledge could be used to create a successful dietary treatment, which may even have the potency to transcend the current results of bariatric surgery. To our knowledge, no such treatment is currently available.

The other independent factor found in Chapter 8 was chronic obstructive pulmonary disease (COPD). Since COPD is highly correlated with smoking, it is unknown which of the two caused the increased risk for postoperative complications. Be that as it may, it is always smart to stop smoking. Furthermore, a pulmonologist should be consulted more routinely to diminish any unnecessary risks intra- and postoperatively.

The next step that should be re-evaluated to reduce the risk of postoperative complications, is the surgical procedure itself. In Chapter 9, the primary RYGB was evaluated. More specifically, the transection or preservation of the neurovascular bundle at the lesser curvature of the stomach was evaluated. The results of this study showed that transection of this neurovascular bundle is associated with an increased risk at major postoperative complications (Clavien-Dindo $\geq 3 \mathrm{~b}$ ) after primary RYGB. As shown in Chapter 9, these results seemed to be independent of the operating surgeon.

Theoretically, these complications are most likely caused by transection of the vein and the artery in the lesser curvature then the abdominal branches of the vagus nerve. Transecting blood vessels does not only mean an increased bleeding risk, but may also contribute to anastomotic ischemia of the gastrojejunostomy, which in turn may cause anastomotic leakage.

It may be quite interesting to know what effect the transection or preservation of these abdominal branches of the vagus nerve will have. Several studies suggest more weight loss in patients undergoing vagotomy or vagal blockade by achieving a faster satiety and reduced calorie intake. ${ }^{54-56}$ Will this affect the long-term outcome of (primary) RYGB or can proper lifestyle changes prevent this from happening? To our knowledge, there is no available evidence on this subject in 2018. Long-term follow-up of the current study population may provide some of these results.

To prevent every single postoperative complication after bariatric surgery is an illusion. One of most difficult treatable complications is leakage. To make it even more complex, there is a wide variety of treatment options. A more 
modern and less invasive method is the use of endoluminal stents, however these stents are confronted with their own limitations such as migration. ${ }^{57-59}$ Chapter 10 reported on the use of a specifically designed for the treatment of anastomotic leakage after bariatric surgery. Despite the specific design, this study showed comparable results when compared to previous literature. ${ }^{60-62}$ The treatment of anastomotic leakages after bariatric surgery remains a challenge. Based on current results when compared to other techniques, stenting should remain an option for the treatment in the future, as $75 \%$ of all leakages was successfully treated. The main goal in stent treatment is to target the high migration rate which was observed in 8 out of 12 patients in the current study. An option might be the more recently introduced lumen apposing stents. ${ }^{63}$

\section{FUTURE PERSPECTIVES}

Bariatric surgery does not only have many clinical and psychological benefits. It can also lead to significant cost savings to health care systems. ${ }^{1}$ Complications after bariatric surgery are known to give a significant increase in health care costs. $^{64,65}$ Reducing the number of complications logically reduces the total health costs.

Not only postoperative complications, but also long-term complications of procedures such as VBG or AGB leads to an increase in total costs. These health care costs are formed by a plurality of components: Properly diagnosing the cause of failure including a proper screening to identify any psychological factors, the increased use of medication to treat complaints, the performance of revisional bariatric surgery when indicated (which in turn has an increased risk at postoperative complications) and many more. In conclusion, the high costs are an additional argument to reduce the number of complications.

Even though many factors have already been identified to contribute to complications in bariatric surgery, new problems could be developing as we speak. Even though the first results of weight loss after primary sleeve gastrectomy seem promising, it is still unknown what the number of long-term complications such as gastroesophageal reflux disease, dysphagia or weight regain will be. ${ }^{29,35,66}$

As more primary RYGB's are performed every year, it is possible that we will be confronted with more patients reporting long-term failure of the current gold standard, the RYGB. ${ }^{67}$ The next challenge will then be to find the most effective treatment depending on the type of failure: Resizing the pouch, banding of the 
gastric bypass, alterations in the limb length or in some cases reversal of the procedure. ${ }^{68-71}$

Furthermore, new procedures such as the SADI may create new cases of failure in the future, which again require the proper treatment.

In conclusion: The search for the perfect bariatric procedure (No complications, sufficient weight loss, no weight regain, no functional complaints, etc.) continues. 


\section{REFERENCES}

1. Borisenko O, Adam D, Funch-Jensen P, Ahmed AR, Zhang R, Colpan Z, et al. Bariatric Surgery can Lead to Net Cost Savings to Health Care Systems: Results from a Comprehensive European Decision Analytic Model. Obes Surg. 2015;25(9):1559-68.

2. Lindekilde N, Gladstone BP, Lubeck M, Nielsen J, Clausen L, Vach W, et al. The impact of bariatric surgery on quality of life: a systematic review and meta-analysis. Obes Rev. 2015; 16(8):639-51.

3. Sjostrom L, Lindroos AK, Peltonen M, Torgerson J, Bouchard C, Carlsson B, et al. Lifestyle, diabetes, and cardiovascular risk factors 10 years after bariatric surgery. N Engl J Med. 2004; 351(26):2683-93.

4. Sjostrom L. Review of the key results from the Swedish Obese Subjects (SOS) trial - a prospective controlled intervention study of bariatric surgery. J Intern Med. 2013;273(3): 219-34.

5. Ortega J, Sala C, Flor B, Jimenez E, Escudero MD, Martinezvalls J, et al. Vertical banded gastroplasty converted to Roux-en-Y gastric bypass: little impact on nutritional status after 5year follow-up. Obes Surg. 2004;14(5):638-43.

6. Balsiger BM, Poggio JL, Mai J, Kelly KA, Sarr MG. Ten and more years after vertical banded gastroplasty as primary operation for morbid obesity. J Gastrointest Surg. 2000;4(6):598-605.

7. Baltasar A, Bou R, Arlandis F, Martinez R, Serra C, Bengochea M, et al. Vertical banded gastroplasty at more than 5 years. Obes Surg. 1998;8(1):29-34.

8. Schouten R, van Dielen FM, van Gemert WG, Greve JW. Conversion of vertical banded gastroplasty to Roux-en-Y gastric bypass results in restoration of the positive effect on weight loss and co-morbidities: evaluation of 101 patients. Obes Surg. 2007;17(5):622-30.

9. Marsk R, Jonas E, Gartzios H, Stockeld D, Granstrom L, Freedman J. High revision rates after laparoscopic vertical banded gastroplasty. Surg Obes Relat Dis. 2009;5(1):94-8.

10. van Rutte PW, Smulders JF, de Zoete JP, Nienhuijs SW. Outcome of sleeve gastrectomy as a primary bariatric procedure. Br J Surg. 2014;101(6):661-8.

11. Buchwald H, Buchwald JN, McGlennon TW. Systematic review and meta-analysis of mediumterm outcomes after banded Roux-en-Y gastric bypass. Obes Surg. 2014;24(9):1536-51.

12. van Hout GC, Hagendoren CA, Verschure SK, van Heck GL. Psychosocial predictors of success after vertical banded gastroplasty. Obes Surg. 2009;19(6):701-7.

13. Mitchell JE, Christian NJ, Flum DR, Pomp A, Pories WJ, Wolfe BM, et al. Postoperative Behavioral Variables and Weight Change 3 Years After Bariatric Surgery. JAMA Surg. 2016;151(8):752-7.

14. Herpertz S, Kielmann R, Wolf AM, Hebebrand J, Senf W. Do psychosocial variables predict weight loss or mental health after obesity surgery? A systematic review. Obes Res. 2004;12(10):1554-69.

15. van Hout GC, Verschure SK, van Heck GL. Psychosocial predictors of success following bariatric surgery. Obes Surg. 2005;15(4):552-60.

16. Wimmelmann CL, Dela F, Mortensen EL. Psychological predictors of mental health and healthrelated quality of life after bariatric surgery: a review of the recent research. Obes Res Clin Pract. 2014;8(4):e314-24.

17. Willcox K, Brennan L. Biopsychosocial outcomes of laparoscopic adjustable gastric banding in adolescents: a systematic review of the literature. Obes Surg. 2014;24(9):1510-9.

18. Cariani S, Agostinelli L, Leuratti L, Giorgini E, Biondi P, Amenta E. Bariatric Revisionary Surgery for Failed or Complicated Vertical Banded Gastroplasty (VBG): Comparison of VBG Reoperation (re-VBG) versus Roux-en-Y Gastric Bypass-on-VBG (RYGB-on-VBG). J Obes. 2010;2010.

19. Foletto $M$, Prevedello $L$, Bernante $P$, Luca B, Vettor $R$, Francini-Pesenti $F$, et al. Sleeve gastrectomy as revisional procedure for failed gastric banding or gastroplasty. Surg Obes Relat Dis. 2010;6(2):146-51. 
20. Gagne DJ, Dovec E, Urbandt JE. Laparoscopic revision of vertical banded gastroplasty to Rouxen-Y gastric bypass: outcomes of 105 patients. Surg Obes Relat Dis. 2011;7(4):493-9.

21. Ardestani A, Lautz DB, Tavakkolizadeh A. Band revision versus Roux-en-Y gastric bypass conversion as salvage operation after laparoscopic adjustable gastric banding. Surg Obes Relat Dis. 2011;7(1):33-7.

22. Carandina S, Maldonado PS, Tabbara M, Valenti A, Rivkine E, Polliand C, et al. Two-step conversion surgery after failed laparoscopic adjustable gastric banding. Comparison between laparoscopic Roux-en-Y gastric bypass and laparoscopic gastric sleeve. Surg Obes Relat Dis. 2014;10(6):1085-91.

23. Lazzati A, De Antonio M, Paolino L, Martini F, Azoulay D, lannelli A, et al. Natural History of Adjustable Gastric Banding: Lifespan and Revisional Rate: A Nationwide Study on Administrative Data on 53,000 Patients. Ann Surg. 2017;265(3):439-45.

24. Mognol P, Chosidow D, Marmuse JP. Laparoscopic conversion of laparoscopic gastric banding to Roux-en-Y gastric bypass: a review of 70 patients. Obes Surg. 2004;14(10):1349-53.

25. Perathoner A, Zitt M, Lanthaler M, Pratschke J, Biebl M, Mittermair R. Long-term follow-up evaluation of revisional gastric bypass after failed adjustable gastric banding. Surg Endosc. 2013;27(11):4305-12.

26. O'Brien PE, MacDonald L, Anderson M, Brennan L, Brown WA. Long-term outcomes after bariatric surgery: fifteen-year follow-up of adjustable gastric banding and a systematic review of the bariatric surgical literature. Ann Surg. 2013;257(1):87-94.

27. Vinzens F, Kilchenmann A, Zumstein V, Slawik M, Gebhart M, Peterli R. Long-term outcome of laparoscopic adjustable gastric banding (LAGB): results of a Swiss single-center study of 405 patients with up to 18 years' follow-up. Surg Obes Relat Dis. 2017;13(8):1313-9.

28. Suter M, Calmes JM, Paroz A, Giusti V. A 10-year experience with laparoscopic gastric banding for morbid obesity: high long-term complication and failure rates. Obes Surg. 2006;16(7): 829-35.

29. Casella G, Soricelli E, Giannotti D, Collalti M, Maselli R, Genco A, et al. Long-term results after laparoscopic sleeve gastrectomy in a large monocentric series. Surg Obes Relat Dis. 2016;12(4):757-62.

30. Edholm D, Svensson F, Naslund I, Karlsson FA, Rask E, Sundbom M. Long-term results 11 years after primary gastric bypass in 384 patients. Surg Obes Relat Dis. 2013;9(5):708-13.

31. Li JF, Lai DD, Lin ZH, Jiang TY, Zhang AM, Dai JF. Comparison of the long-term results of Rouxen-Y gastric bypass and sleeve gastrectomy for morbid obesity: a systematic review and meta-analysis of randomized and nonrandomized trials. Surg Laparosc Endosc Percutan Tech. 2014;24(1):1-11.

32. Dogan K, Kraaij L, Aarts EO, Koehestanie P, Hammink E, van Laarhoven CJ, et al. Fast-track bariatric surgery improves perioperative care and logistics compared to conventional care. Obes Surg. 2015;25(1):28-35.

33. Geubbels N, Bruin SC, Acherman YI, van de Laar AW, Hoen MB, de Brauw LM. Fast track care for gastric bypass patients decreases length of stay without increasing complications in an unselected patient cohort. Obes Surg. 2014;24(3):390-6.

34. Cordera F, Mai JL, Thompson GB, Sarr MG. Unsatisfactory weight loss after vertical banded gastroplasty: is conversion to Roux-en-Y gastric bypass successful? Surgery. 2004;136(4): 731-7.

35. Gautier T, Sarcher T, Contival N, Le Roux Y, Alves A. Indications and mid-term results of conversion from sleeve gastrectomy to Roux-en-Y gastric bypass. Obes Surg. 2013;23(2): 212-5.

36. Marin-Perez P, Betancourt A, Lamota M, Lo Menzo E, Szomstein S, Rosenthal R. Outcomes after laparoscopic conversion of failed adjustable gastric banding to sleeve gastrectomy or Roux-en-Y gastric bypass. Br J Surg. 2014;101(3):254-60.

37. lannelli $A$, Schneck AS, Ragot $E$, Liagre A, Anduze $Y$, Msika $S$, et al. Laparoscopic sleeve gastrectomy as revisional procedure for failed gastric banding and vertical banded gastroplasty. Obes Surg. 2009;19(9):1216-20. 
38. van Rutte PW, Smulders JF, de Zoete JP, Nienhuijs SW. Indications and short-term outcomes of revisional surgery after failed or complicated sleeve gastrectomy. Obes Surg. 2012; 22(12):1903-8.

39. Shikora SA, Kim JJ, Tarnoff ME, Raskin E, Shore R. Laparoscopic Roux-en-Y gastric bypass: results and learning curve of a high-volume academic program. Arch Surg. 2005;140(4):362-7.

40. Stepaniak PS, Heij C, Buise MP, Mannaerts GH, Smulders JF, Nienhuijs SW. Bariatric surgery with operating room teams that stayed fixed during the day: a multicenter study analyzing the effects on patient outcomes, teamwork and safety climate, and procedure duration. Anesth Analg. 2012;115(6):1384-92.

41. Parmar CD, Mahawar KK, Boyle M, Schroeder N, Balupuri S, Small PK. Conversion of Sleeve Gastrectomy to Roux-en-Y Gastric Bypass is Effective for Gastro-Oesophageal Reflux Disease but not for Further Weight Loss. Obes Surg. 2017;27(7):1651-8.

42. Casillas RA, Um SS, Zelada Getty JL, Sachs S, Kim BB. Revision of primary sleeve gastrectomy to Roux-en-Y gastric bypass: indications and outcomes from a high-volume center. Surg Obes Relat Dis. 2016;12(10):1817-25.

43. Yorke E, Sheppard C, Switzer NJ, Kim D, de Gara C, Karmali S, et al. Revision of sleeve gastrectomy to Roux-en-Y Gastric Bypass: A Canadian experience. Am J Surg. 2017;213(5): 970-4.

44. Carmeli I, Golomb I, Sadot E, Kashtan H, Keidar A. Laparoscopic conversion of sleeve gastrectomy to a biliopancreatic diversion with duodenal switch or a Roux-en-Y gastric bypass due to weight loss failure: our algorithm. Surg Obes Relat Dis. 2015;11(1):79-85.

45. Homan J, Betzel B, Aarts EO, van Laarhoven KJ, Janssen IM, Berends FJ. Secondary surgery after sleeve gastrectomy: Roux-en-Y gastric bypass or biliopancreatic diversion with duodenal switch. Surg Obes Relat Dis. 2015;11(4):771-7.

46. Sanchez-Pernaute A, Rubio MA, Conde M, Arrue E, Perez-Aguirre E, Torres A. Singleanastomosis duodenoileal bypass as a second step after sleeve gastrectomy. Surg Obes Relat Dis. 2015;11(2):351-5.

47. Sanchez-Pernaute A, Rubio MA, Perez Aguirre E, Barabash A, Cabrerizo L, Torres A. Singleanastomosis duodenoileal bypass with sleeve gastrectomy: metabolic improvement and weight loss in first 100 patients. Surg Obes Relat Dis. 2013;9(5):731-5.

48. Dijkhorst PJ, Boerboom AB, Janssen IMC, Swank DJ, Wiezer RMJ, Hazebroek EJ, et al. Failed Sleeve Gastrectomy: Single Anastomosis Duodenoileal Bypass or Roux-en-Y Gastric Bypass? A Multicenter Cohort Study. Obes Surg. 2018;28(12):3834-42.

49. Moon RC, Fuentes AS, Teixeira AF, Jawad MA. Conversions After Sleeve Gastrectomy for Weight Regain: to Single and Double Anastomosis Duodenal Switch and Gastric Bypass at a Single Institution. Obes Surg. 2019;29(1):48-53.

50. Wu A, Tian J, Cao L, Gong F, Wu A, Dong G. Single-anastomosis duodeno-ileal bypass with sleeve gastrectomy (SADI-S) as a revisional surgery. Surg Obes Relat Dis. 2018;14(11):1686-90.

51. Inabnet WB, 3rd, Winegar DA, Sherif B, Sarr MG. Early outcomes of bariatric surgery in patients with metabolic syndrome: an analysis of the bariatric outcomes longitudinal database. J Am Coll Surg. 2012;214(4):550-6; discussion 6-7.

52. Lau E, Carvalho D, Pina-Vaz C, Barbosa JA, Freitas P. Beyond gut microbiota: understanding obesity and type 2 diabetes. Hormones (Athens). 2015;14(3):358-69.

53. Ussar S, Griffin NW, Bezy O, Fujisaka S, Vienberg S, Softic S, et al. Interactions between Gut Microbiota, Host Genetics and Diet Modulate the Predisposition to Obesity and Metabolic Syndrome. Cell Metab. 2015;22(3):516-30.

54. Kral JG, Gortz L, Hermansson G, Wallin GS. Gastroplasty for obesity: long-term weight loss improved by vagotomy. World J Surg. 1993;17(1):75-8; discussion 9.

55. Sarr MG, Billington CJ, Brancatisano R, Brancatisano A, Toouli J, Kow L, et al. The EMPOWER study: randomized, prospective, double-blind, multicenter trial of vagal blockade to induce weight loss in morbid obesity. Obes Surg. 2012;22(11):1771-82. 
56. Camilleri M, Toouli J, Herrera MF, Kulseng B, Kow L, Pantoja JP, et al. Intra-abdominal vagal blocking (VBLOC therapy): clinical results with a new implantable medical device. Surgery. 2008;143(6):723-31

57. Leenders BJ, Stronkhorst A, Smulders FJ, Nieuwenhuijzen GA, Gilissen LP. Removable and repositionable covered metal self-expandable stents for leaks after upper gastrointestinal surgery: experiences in a tertiary referral hospital. Surg Endosc. 2013;27(8):2751-9.

58. Salminen $P$, Gullichsen $R$, Laine $S$. Use of self-expandable metal stents for the treatment of esophageal perforations and anastomotic leaks. Surg Endosc. 2009;23(7):1526-30.

59. van Boeckel PG, Sijbring A, Vleggaar FP, Siersema PD. Systematic review: temporary stent placement for benign rupture or anastomotic leak of the oesophagus. Aliment Pharmacol Ther. 2011;33(12):1292-301.

60. Eisendrath P, Cremer M, Himpens J, Cadiere GB, Le Moine O, Deviere J. Endotherapy including temporary stenting of fistulas of the upper gastrointestinal tract after laparoscopic bariatric surgery. Endoscopy. 2007;39(7):625-30.

61. Eubanks S, Edwards CA, Fearing NM, Ramaswamy A, de la Torre RA, Thaler KJ, et al. Use of endoscopic stents to treat anastomotic complications after bariatric surgery. J Am Coll Surg. 2008;206(5):935-8; discussion 8-9.

62. Puli SR, Spofford IS, Thompson CC. Use of self-expandable stents in the treatment of bariatric surgery leaks: a systematic review and meta-analysis. Gastrointest Endosc. 2012;75(2):287-93.

63. Mansoor MS, Tejada J, Parsa NA, Yoon E, Hida S. Off label use of lumen-apposing metal stent for persistent gastro-jejunal anastomotic stricture. World J Gastrointest Endosc. 2018;10(6): $117-20$.

64. Bransen J, Gilissen LP, van Rutte PW, Nienhuijs SW. Costs of Leaks and Bleeding After Sleeve Gastrectomies. Obes Surg. 2015;25(10):1767-71.

65. Shah N, Greenberg JA, Leverson G, Funk LM. Predictors of high cost after bariatric surgery: A single institution review. Surgery. 2016;160(4):877-84.

66. Genco A, Soricelli E, Casella G, Maselli R, Castagneto-Gissey L, Di Lorenzo N, et al. Gastroesophageal reflux disease and Barrett's esophagus after laparoscopic sleeve gastrectomy: a possible, underestimated long-term complication. Surg Obes Relat Dis. 2017; 13(4):568-74.

67. Cazzo E, da Silva FP, Pareja JC, Chaim EA. Predictors for weight loss failure following Roux-enY gastric bypass. Arq Gastroenterol. 2014;51(4):328-30.

68. Ferraz AA, de Siqueira LT, Nunes Filho E, de Araujo JG, Jr., Campos JM, de Barros-Correia TX, et al. Revision surgery for treatment of weight regain after Roux-en- $Y$ gastric bypass. Obes Surg. 2014;24(1):2-8.

69. Heneghan HM, Annaberdyev S, Eldar S, Rogula T, Brethauer S, Schauer P. Banded Roux-en-Y gastric bypass for the treatment of morbid obesity. Surg Obes Relat Dis. 2014;10(2):210-6.

70. lannelli A, Schneck AS, Hebuterne $X$, Gugenheim J. Gastric pouch resizing for Roux-en-Y gastric bypass failure in patients with a dilated pouch. Surg Obes Relat Dis. 2013;9(2):260-7.

71. Zaveri H, Dallal RM, Cottam D, Surve A, Kartiko S, Bonnani F, et al. Indications and Operative Outcomes of Gastric Bypass Reversal. Obes Surg. 2016;26(10):2285-90. 



\section{CHAPTER 13}

Nederlandse samenvatting
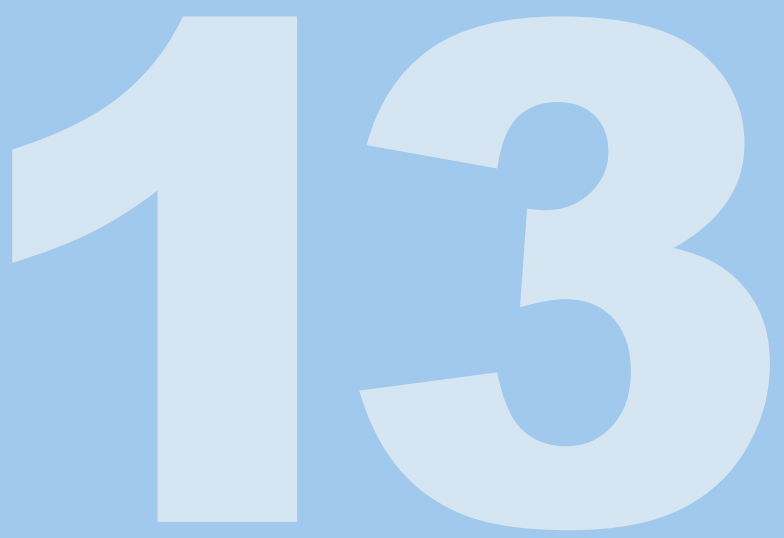



\section{NEDERLANDSE SAMENVATTING}

De gevolgen van (morbide) obesitas zijn enorm. Obesitas heeft een grote impact op iemands leven en kan leiden tot vroege sterfte, chronische ziekten en hoge gezondheidskosten. Er wordt gesproken over obesitas indien de Body Mass Index (BMI) groter is dan $30 \mathrm{~kg} / \mathrm{m}^{2}$. Er wordt zelfs gesproken van een pandemie. De laatste cijfers van de World Health Organization (WHO) rapporteren dat $13 \%$ van de wereldbevolking obees was in 2016, wat neerkomt op ongeveer 650 miljoen mensen wereldwijd. ${ }^{1}$ Een substantieel deel van deze groep mensen heeft zelfs morbide obesitas. Er wordt gesproken van morbide obesitas bij een $\mathrm{BMI} \geq 40$ of een $\mathrm{BMI} \geq 35$ met klachten van een of meerdere obesitas gerelateerde aandoeningen. Deze aandoeningen zijn onder andere: diabetes mellitus type 2 (suikerziekte), hypertensie (hoge bloeddruk), hypercholesterolemie (hoog cholesterol), obstructief slaap apnoe syndroom (OSAS, adempauzes tijdens de slaap) en gewrichtsklachten.

Er bestaan veel behandelmogelijkheden voor morbide obesitas, maar momenteel is bariatrische chirurgie (chirurgie met gewichtsverlies als doel) de enige effectieve behandeling op de lange termijn. ${ }^{2}$ Op de lange termijn zorgt het voor een totale gewichtsafname van $16-23 \%$ en in een groot deel van de gevallen voor de genezing van obesitas gerelateerde aandoeningen. ${ }^{2-5}$

Om uiteenlopende redenen kan een operatie niet het gewenste effect hebben. Er wordt dan gesproken van een complicatie op de lange termijn. Deze complicaties zijn divers; zo kan een patiënt te veel zijn afgevallen, te weinig zijn afgevallen (ook wel een primaire non-responder genoemd) of na goed af te zijn gevallen weer aankomen in gewicht (secundaire non-responder). Daarnaast kan er sprake zijn van blijvende functionele problemen, zoals slikproblemen, maagzuurklachten of voedselintolerantie. In een deel van deze gevallen dient de eerdere procedure te worden omgezet door middel van een nieuwe operatie. Dit wordt ook wel revisiechirurgie of redo-chirurgie genoemd. Als een oude operatie wordt omgezet, wordt er tegenwoordig ook wel gesproken van een conversie. Bijvoorbeeld: $\mathrm{Er}$ is een maagband geplaatst met aanvankelijk een goed resultaat. Na een aantal jaar komt de patiënt toch terug omdat er complicaties zijn van de maagband. Er kan dan voor gekozen worden om de band te verwijderen en een gastric bypass uit te voeren. In andere gevallen kan ervoor gekozen worden de bestaande ingreep te reviseren (herzien).

Naast de zojuist genoemde nadelen op de lange termijn (waarbij in een deel van de gevallen revisiechirurgie noodzakelijk is), kunnen er ook complicaties optreden vlak na de operatie. Vaak zijn dit complicaties binnen 30 dagen na de operatie. Zo kan er bijvoorbeeld een nabloeding, een wondinfectie of een naadlekkage optreden. 
Dit proefschrift richt zich op deze verschillende complicaties. Het proefschrift is opgesplitst in twee delen. Het eerste deel richt zich op de revisiechirurgie als behandeling van de lange termijncomplicaties na eerdere bariatrische chirurgie. Er wordt meer inzicht verkregen in zowel de gefaalde oude operaties met hun lange termijn complicaties, als in de uitkomsten en veiligheid van de hierop volgende revisieoperaties. Het tweede deel richt zich op de korte termijncomplicaties na een gastric bypass (maagomleiding), waarbij gepoogd is om met diverse methoden de complicaties te verminderen en te behandelen.

\section{Deel een}

Revisiechirurgie wordt vooral uitgevoerd als behandeling van een lange termijncomplicatie van een eerdere maagverkleinende ingreep. Om de redenen voor revisiechirurgie beter te kunnen begrijpen, is het essentieel om te begrijpen wat de effecten van de originele bariatrische ingreep zijn. In Hoofdstuk 2 worden de lange termijnresultaten besproken van de vertical banded gastroplasty (VBG), ook wel bekend als Mason-gastroplastiek, een oudere vorm van obesitaschirurgie welke in de jaren ' 80 is geïntroduceerd. Met gemiddeld $53 \%$ verlies van het overtollige gewicht (ook wel bekend als excess weight loss; EWL) en een verbetering bij 55\% van obesitas gerelateerde aandoeningen (diabetes mellitus type 2, hypertensie, hypercholesterolemie, obstructief slaap apnoe syndroom en gewrichtsklachten) zijn de resultaten na een VBG acceptabel te noemen. Het grote nadeel van de VBG wordt echter ook aangetoond in dit hoofdstuk. Maar liefst 58\% van de patiënten heeft klachten ontwikkeld na de operatie. Bijna 40\% van de onderzochte populatie heeft uiteindelijk een revisieoperatie ondergaan. Dit hoge percentage aan lange termijncomplicaties onderstreept waarom de VBG momenteel niet meer wordt uitgevoerd in Nederland.

De afgelopen decennia is het in toenemende mate duidelijk geworden dat het succes van bariatrische chirurgie ook afhankelijk is van verschillende psychologische factoren, zoals het eetgedrag van een patiënt. ${ }^{6}$ Hoofdstuk $\mathbf{3}$ is erop gericht om factoren te identificeren die een goede of juist een slechte uitkomst voorspellen. De uitkomst wordt bepaald door de hoeveelheid gewichtsverlies, waarbij minder dan $50 \%$ verlies van het overtollige gewicht als een slechte uitkomst wordt beschouwd. In deze populatie worden een aantal factoren geïdentificeerd als voorspeller van een slechte uitkomst na bariatrische chirurgie. Zo blijkt in de onderzochte populatie dat patiënten met esthetisch hogere verwachtingen minder goed afvielen op de lange termijn. Daarnaast was de hoeveelheid werkverzuim vóór de operatie hoger in deze groep en werd er een hogere afhankelijkheid gezien van de operatie. Dit houdt in andere 
woorden in dat deze patiënten meer vertrouwen op het effect van de operatie dan het effect van de leefstijlveranderingen die noodzakelijk zijn. ${ }^{7}$

Zoals al eerder gemeld is het revisiepercentage hoog na een VBG. Omdat de literatuur verschillende opties beschrijft als revisieoperatie van een eerdere VBG, wordt in Hoofdstuk 4 gekeken welke optie het beste lijkt te zijn in een retrospectieve analyse. De 3 opties die werden onderzocht zijn het herzien van de originele operatie (revisie van de $V B G$ ), omzetting naar een sleeve gastrectomie (SG) of een Roux-en-Y gastric bypass (RYGB). De resultaten in deze studie laten duidelijk zien dat de beste revisieoperatie de conversie naar een RYGB is. Deze optie laat namelijk het hoogste gewichtsverlies op de lange termijn, de meeste verbetering van obesitas gerelateerde aandoeningen en de laagste incidentie lange termijncomplicaties zien in vergelijking met de andere twee opties. Gebaseerd op de resultaten uit deze studie wordt dan ook geadviseerd om in het geval van een gefaalde VBG te kiezen voor een omzetting naar RYGB, tenzij dit technisch niet mogelijk blijkt te zijn.

In Hoofdstuk 5 wordt de hypothese onderzocht of omzetting van een gefaalde maagband, ofwel adjustable gastric band (AGB), naar een RYGB gepaard gaat met een hoger percentage postoperatieve complicaties in vergelijking de omzetting van een gefaalde SG naar een RYGB. Dit zou een extra argument kunnen zijn om de $A G B$ als primaire bariatrische ingreep te verlaten, naast de bekende hoge percentages lange termijncomplicaties. ${ }^{8-10}$ De resultaten laten echter vergelijkbare resultaten zien tussen de twee groepen (omzetting AGB naar RYGB versus omzetting SG naar RYGB). Zo is er een vergelijkbaar percentage postoperatieve complicaties (8.8\% versus $11.8 \% ; \quad p=0.530)$, gewichtsverlies en verbetering van obesitas-gerelateerde aandoeningen. Wordt er extra zorgvuldig naar deze resultaten gekeken, dan valt op dat het totale gewichtsverlies in de SG-groep op de lange termijn iets afneemt, in tegenstelling tot de AGB-groep, waar dit juist licht toeneemt. De conclusie uit Hoofdstuk 5 is dat conversie naar een RYGB een goede revisieprocedure is voor een gefaalde AGB. Dit statement kan niet zo hard gemaakt worden voor de omzetting van een gefaalde SG naar RYGB op basis van deze resultaten. Op basis van (extra) gewichtsverlies lijken er andere opties de voorkeur te krijgen, zoals de biliopancreatic diversion met duodenal switch (BPD-DS) of een single anastomosis duodenoileal bypass (SADI, omleiding door middel van één aansluiting van de dunne darm op de twaalfvingerige darm), twee nieuwe types van bariatrische operaties. ${ }^{11,12}$

Het aantal bariatrische ingrepen neemt wereldwijd nog steeds toe, waarbij de behoefte aan een fast track protocol (programma gericht op een versneld herstel na een operatie) ook steeds verder toeneemt. ${ }^{13}$ Onderzoek heeft aangetoond dat dit veilig is in primaire bariatrische chirurgie. ${ }^{14-16}$ Met in 2016 
bijna 51.000 verrichte revisieprocedures wereldwijd, een aantal dat nog steeds toeneemt, kan dit echter ook voordelen bieden bij revisiechirurgie. ${ }^{13}$ Daarom wordt in Hoofdstuk $\mathbf{6}$ de veiligheid onderzocht van een fast track protocol bij patiënten die een technisch veel lastigere bariatrische revisieprocedure ondergaan. Dit retrospectieve onderzoek rapporteert een significant lager complicatiepercentage sinds de invoer van het fast track protocol bij bariatrische revisiechirurgie. Hierbij moet de kanttekening gemaakt wordt dat deze percentages niet alleen beïnvloed zullen zijn door het fast track protocol, maar mogelijk ook door een toegenomen ervaring van het operatieteam, de leercurve van de chirurg en verbeterde chirurgische apparatuur. Desalniettemin mag geconcludeerd worden dat de implementatie van een fast track protocol bij bariatrische revisiechirurgie veilig is.

Zoals reeds vernoemd in Hoofdstuk 5, is het extra gewichtsverlies na een omzetting van een gefaalde SG naar RYGB beperkt. Er lijken betere alternatieven beschikbaar waaronder de BPD-DS en de SADI, maar het bewijs in de literatuur is beperkt. ${ }^{11,12,17}$ In Hoofdstuk 7 worden in een matched cohort studie de uitkomsten van de SADI als alternatieve revisieprocedure na een gefaalde SG vergeleken met die van de RYGB. Getalsmatig toont dit een iets hoger gewichtsverlies en minder postoperatieve complicaties in de SADI groep wanneer deze vergeleken wordt met de RYGB groep, maar deze verschillen zijn niet statistisch significant. Op basis van deze resultaten kan wel voorzichtig gesteld worden dat de SADI als revisieprocedure minder complicaties lijkt te geven in vergelijking met de beschikbare percentages na BPD-DS. Andere studies suggereren ook een gunstig effect van de SADI als revisieprocedure na een gefaalde SG. ${ }^{18-20}$ Het grootste nadeel van deze resultaten is het retrospectieve karakter van de studies. Goed prospectief, gerandomiseerd onderzoek is nodig om de kennis van de SADI als revisieprocedure na een gefaalde SG uit te breiden. Mede daarom wordt in Hoofdstuk 8 een protocolvoorstel voor een prospectieve randomized controlled trial die de effecten vergelijkt tussen de SADI en de RYGB als revisieprocedure na een eerdere SG.

\section{Deel twee}

De focus van deel twee van dit proefschrift lag op de vroege complicaties na bariatrische chirurgie. Er wordt over het algemeen gesproken van een vroege complicatie indien deze binnen 30 dagen na de operatie optreedt. Veel onderzoek is al uitgevoerd om het percentage vroege complicaties tot een minimum te beperken, maar er is nog steeds ruimte voor verbetering. Preventie is van belang, omdat bij (morbide) obesitaspatiënten het beloop van een complicatie veel ernstiger kan zijn. 
Deze vroege complicaties worden vaak gegradeerd naar ernst van de complicatie met behulp van de Clavien-Dindo classificatie. ${ }^{21}$ Deze classificatie beschrijft de ernst van de complicatie van graad I (simpele wondinfectie) tot aan graad $V$ (dood van een patiënt). Hoofdstukken 9 en 10 zullen zich focussen op de complicaties van graad IIla (complicatie waarvoor een radiologische, endoscopische of chirurgische interventie zonder algehele narcose noodzakelijk is) en hoger; ook wel serieuze complicaties of 'serious adverse events' genoemd.

Om de hoeveelheid serieuze complicaties te verlagen lijkt preventie het beste middel. Voor een adequate preventie is het identificeren van risicofactoren noodzakelijk. Hoofdstuk 9 beschrijft een aantal van deze risicofactoren die gerelateerd zijn aan het ontstaan van serieuze postoperatieve complicaties waarvoor minstens een re-interventie (radiologisch, endoscopisch of chirurgisch) noodzakelijk is (classificatie volgens Clavien-Dindo $\geq 3 \mathrm{a}$ ). In een populatie van 773 patiënten waarbij een primaire RYGB werd uitgevoerd zijn 2 onafhankelijke risicofactoren geïdentificeerd: het mannelijk geslacht (Gemiddeld een 2,4 keer hogere kans op een complicatie ten opzichte van het vrouwelijk geslacht) en COPD (chronic obstructive pulmonary disease; gemiddeld een 3,7 keer hogere kans op een complicatie ten opzichte van patiënten zonder COPD).

Een van de hypotheses waardoor deze complicaties ontstaan, in dit geval specifiek een naadlekkage, is het doornemen van de neurovasculaire bundel bij de maag, bestaande uit de bloedvoorziening en aftakkingen van de nervus vagus (een van de 12 zogenaamde hersenzenuwen). Dit doornemen is noodzakelijk om de gastrojejunostomie te creëren; de aansluiting tussen de nieuwe (kleinere) maag en de darm. Hierdoor zou een verhoogd bloedingsrisico kunnen ontstaan. Ook zou dit kunnen leiden tot een verhoogd risico op ischemie van de nieuw aangelegde gastrojejunostomie, wat vervolgens weer kans geeft op een naadlekkage. In Hoofdstuk $\mathbf{1 0}$ wordt in een retrospectieve analyse het effect vergeleken van twee technieken: het sparen van deze neurovasculaire bundel versus het doornemen van deze neurovasculaire bundel. De resultaten laten een statistisch significant lager percentage serieuze complicaties zien (Clavien-Dindo $\geq 3 a$ ) in de groep patiënten waarbij de neurovasculaire bundel wordt gespaard. In deze studie lijkt dit resultaat niet af te hangen van de opererend chirurg. In deze studie is niet onderzocht wat het effect van deze twee technieken heeft op de lange termijn in het kader van mogelijke passageklachten, dumpingklachten en het gewichtsverlies.

Een van de lastigst te behandelen korte termijncomplicaties is een naadlekkage. Het is een complicatie die maakt dat patiënten ernstig ziek kunnen worden (sepsis), gaat regelmatig gepaard met het ontstaan van abcessen in de 
buikholte en vraagt eigenlijk altijd om een re-interventie (chirurgisch, endoscopisch of radiologisch). De aanpak van dit probleem wordt bemoeilijkt door de grote variatie aan behandelopties. Een modernere en minder invasieve methode voor de behandeling van een naadlekkage is het gebruik van endoluminale stents (stents die geplaatst worden aan de binnenzijde van maag of darmen). Een belangrijk nadeel van deze stents is migratie. Hoofdstuk $\mathbf{1 1}$ beschrijft de resultaten van een specifiek ontworpen stent om dit probleem te voorkomen. De resultaten tonen echter dat ook dit specifieke ontwerp het probleem van stentmigratie (66.7\%) niet kan voorkomen en dat het succesvol behandelpercentage van $75 \%$ vergelijkbaar is met eerdere literatuur. ${ }^{22-24}$

In dit proefschrift zijn zowel korte als lange termijncomplicaties onderzocht en daarbij behorende behandelopties. De gevonden resultaten en conclusies leiden tot meer inzicht in welke factoren invloed hebben op de uitkomsten na bariatrische chirurgie. Zij dragen daarmee hopelijk bij aan een betere behandelstrategie welke zal moeten zorgen voor betere uitkomsten na bariatrische (revisie)chirurgie met een verlaging van het aantal korte en lange termijncomplicaties. 


\section{REFERENCES}

1. WHO. Obesity and overweight fact sheet 2018 [updated February 2018; cited 2019 23-032019]. Available from: http://www.who.int/mediacentre/factsheets/fs311/en/.

2. Sjostrom L, Lindroos AK, Peltonen M, Torgerson J, Bouchard C, Carlsson B, et al. Lifestyle, diabetes, and cardiovascular risk factors 10 years after bariatric surgery. N Engl J Med. 2004; 351(26):2683-93.

3. Sjostrom L. Review of the key results from the Swedish Obese Subjects (SOS) trial - a prospective controlled intervention study of bariatric surgery. J Intern Med. 2013;273(3): 219-34.

4. Sjostrom L, Gummesson A, Sjostrom CD, Narbro K, Peltonen M, Wedel H, et al. Effects of bariatric surgery on cancer incidence in obese patients in Sweden (Swedish Obese Subjects Study): a prospective, controlled intervention trial. Lancet Oncol. 2009;10(7):653-62.

5. Sjostrom L, Narbro K, Sjostrom CD, Karason K, Larsson B, Wedel H, et al. Effects of bariatric surgery on mortality in Swedish obese subjects. N Engl J Med. 2007;357(8):741-52.

6. van Hout GC, Hagendoren CA, Verschure SK, van Heck GL. Psychosocial predictors of success after vertical banded gastroplasty. Obes Surg. 2009;19(6):701-7.

7. Kalarchian $M$, Turk $M$, Elliott J, Gourash W. Lifestyle management for enhancing outcomes after bariatric surgery. Curr Diab Rep. 2014;14(10):540.

8. O'Brien PE, MacDonald L, Anderson M, Brennan L, Brown WA. Long-term outcomes after bariatric surgery: fifteen-year follow-up of adjustable gastric banding and a systematic review of the bariatric surgical literature. Ann Surg. 2013;257(1):87-94.

9. Lazzati A, De Antonio M, Paolino L, Martini F, Azoulay D, lannelli A, et al. Natural History of Adjustable Gastric Banding: Lifespan and Revisional Rate: A Nationwide Study on Administrative Data on 53,000 Patients. Ann Surg. 2017;265(3):439-45.

10. Vinzens F, Kilchenmann A, Zumstein V, Slawik M, Gebhart M, Peterli R. Long-term outcome of laparoscopic adjustable gastric banding (LAGB): results of a Swiss single-center study of 405 patients with up to 18 years' follow-up. Surg Obes Relat Dis. 2017;13(8):1313-9.

11. Homan J, Betzel B, Aarts EO, van Laarhoven KJ, Janssen IM, Berends FJ. Secondary surgery after sleeve gastrectomy: Roux-en-Y gastric bypass or biliopancreatic diversion with duodenal switch. Surg Obes Relat Dis. 2015;11(4):771-7.

12. Sanchez-Pernaute A, Rubio MA, Conde $M$, Arrue E, Perez-Aguirre E, Torres A. Singleanastomosis duodenoileal bypass as a second step after sleeve gastrectomy. Surg Obes Relat Dis. 2015;11(2):351-5.

13. Angrisani L, Santonicola A, lovino P, Vitiello A, Higa K, Himpens J, et al. IFSO Worldwide Survey 2016: Primary, Endoluminal, and Revisional Procedures. Obes Surg. 2018;28(12): 3783-94.

14. Dogan K, Kraaij L, Aarts EO, Koehestanie P, Hammink E, van Laarhoven CJ, et al. Fast-track bariatric surgery improves perioperative care and logistics compared to conventional care. Obes Surg. 2015;25(1):28-35.

15. Geubbels N, Bruin SC, Acherman YI, van de Laar AW, Hoen MB, de Brauw LM. Fast track care for gastric bypass patients decreases length of stay without increasing complications in an unselected patient cohort. Obes Surg. 2014;24(3):390-6.

16. Stepaniak PS, Heij C, Buise MP, Mannaerts GH, Smulders JF, Nienhuijs SW. Bariatric surgery with operating room teams that stayed fixed during the day: a multicenter study analyzing the effects on patient outcomes, teamwork and safety climate, and procedure duration. Anesth Analg. 2012;115(6):1384-92.

17. Carmeli I, Golomb I, Sadot E, Kashtan H, Keidar A. Laparoscopic conversion of sleeve gastrectomy to a biliopancreatic diversion with duodenal switch or a Roux-en-Y gastric bypass due to weight loss failure: our algorithm. Surg Obes Relat Dis. 2015;11(1):79-85. 
18. Dijkhorst PJ, Boerboom AB, Janssen IMC, Swank DJ, Wiezer RMJ, Hazebroek EJ, et al. Failed Sleeve Gastrectomy: Single Anastomosis Duodenoileal Bypass or Roux-en-Y Gastric Bypass? A Multicenter Cohort Study. Obes Surg. 2018;28(12):3834-42.

19. Moon RC, Fuentes AS, Teixeira AF, Jawad MA. Conversions After Sleeve Gastrectomy for Weight Regain: to Single and Double Anastomosis Duodenal Switch and Gastric Bypass at a Single Institution. Obes Surg. 2018.

20. Wu A, Tian J, Cao L, Gong F, Wu A, Dong G. Single-anastomosis duodeno-ileal bypass with sleeve gastrectomy (SADI-S) as a revisional surgery. Surg Obes Relat Dis. 2018.

21. Dindo D, Demartines N, Clavien PA. Classification of surgical complications: a new proposal with evaluation in a cohort of 6336 patients and results of a survey. Annals of surgery. 2004;240(2):205-13.

22. Eisendrath P, Cremer M, Himpens J, Cadiere GB, Le Moine O, Deviere J. Endotherapy including temporary stenting of fistulas of the upper gastrointestinal tract after laparoscopic bariatric surgery. Endoscopy. 2007;39(7):625-30.

23. Eubanks S, Edwards CA, Fearing NM, Ramaswamy A, de la Torre RA, Thaler KJ, et al. Use of endoscopic stents to treat anastomotic complications after bariatric surgery. J Am Coll Surg. 2008;206(5):935-8; discussion 8-9.

24. Puli SR, Spofford IS, Thompson CC. Use of self-expandable stents in the treatment of bariatric surgery leaks: a systematic review and meta-analysis. Gastrointest Endosc. 2012;75(2):287-93. 
PART THREE

Appendices 

Valorisation 



\section{VALORISATION}

This thesis describes the outcome and safety of revisional surgery as treatment option for long-term complications after primary bariatric surgery, while gaining more knowledge on the older bariatric procedures. Furthermore, this thesis describes some methods to reduce and properly treat short-term complications such as anastomotic leakage. Bariatric surgery is still gaining in popularity, as is shown by the increasing number of bariatric procedures performed annually worldwide. ${ }^{1-4}$

In 2018 in the Netherlands, a total of 11,468 procedures were performed, of which roughly $10 \%$ were revisional procedures. At the end of 2018 , Angrisani et al. published the global numbers of 2016, describing a total number of 685,784 procedures of which $7.4 \%(n=50,977)$ were revisional procedures. This was the first manuscript to separately describe the worldwide number of revisional procedures, which strengthens the believe that revisional surgery is obtaining a larger part in the diverse world of bariatric surgery. ${ }^{1-3}$

More knowledge is becoming available on the large amount of long-term complications after bariatric surgery, which often required revisional surgery. Even though the older procedures and their complications are rapidly decreasing in volume, a lot is still unknown about these long-term complications in terms of the mechanism of origin, the proper treatment and the risk factors contributing to the occurrence of these complications. Furthermore, more data is needed on the effects and safety of revisional bariatric procedures and all the gathered data will be useful for any future complications in modern or future bariatric procedures. The first part of this thesis provides some support on these issues, which will be discussed below.

Even though bariatric surgery is relatively safe, the high number of performed procedures worldwide results in a high absolute number of complications. ${ }^{5-7}$ The second part focuses on the challenging issue of lowering the early serious complication rate after primary Roux-en-Y gastric bypass (RYGB) surgery to an all-time low.

Below, the value of this thesis in discussed in separate sections.

\section{Social and economic relevance}

Bransen et al. have reported on the additional health care costs of leaks and bleedings after sleeve gastrectomy. ${ }^{8}$ This study reported high costs after a serious complication. These costs were especially high due to prolonged hospitalization and additional interventions.

These numbers should be taken into account when looking at the total number of annually performed bariatric procedures. In case of these large numbers, 
even less than one percent reduction in serious complications will cause a significant reduction in total health care costs. Therefore, this thesis has provided data on how to further reduce these serious complications.

The amount of long-term complications is largely dependent on choosing the right primary bariatric procedure. Even though this thesis does not provide a definitive answer on what the best procedure is, it supports abandoning VBG as primary procedure and speculates on the indication of $A G B$ as primary procedure, as it shows a high number of long-term complications. This thesis seems to support the choice for conversion to RYGB in case of failed VBG or $A G B$, although new alternatives should be explored as well.

Such information has to be taken into account in the decision-making process for the revisional-technique of choice. This in turn will help reducing health care costs as the patient is becoming healthier. ${ }^{9}$

Finally, this thesis has proven that the implementation of a fast track protocol is safe. This protocol potentially reduces health care costs by lowering hospital stay and improving logistics without increasing the complication rate. ${ }^{10,11}$

\section{Target audience}

This thesis targets a broad audience as it contains valuable information for bariatric surgeons, obesity teams, general practitioners (who will eventually perform follow-up of these patients) and other specialists such as endocrinologists and gastroenterologists. This thesis attempts to provide some unity to bariatric experts in treating long-term complications which necessitate revisional bariatric surgery.

All patients currently experiencing complications after restrictive procedures such as primary vertical banded gastroplasty (VBG) or adjustable gastric banding (AGB) can be helped more accurately with the use of the results in this thesis, as it provides some guidelines for clinicians who are being challenged by long-term complications after VBG or AGB.

In case of failed VBG of $A G B$, this thesis advises to perform a conversion to RYGB when revisional surgery is deemed necessary. Furthermore, VBG should be completely abandoned as primary bariatric procedure. In case of a failed sleeve gastrectomy, more research is required, since this thesis challenges the gold standard of performing conversion to RYGB after failed SG and provides a potentially better alternative which is the Single Anastomosis Duodenolleal bypass (SADI).

Furthermore, this thesis urges all clinicians to always preserve the neurovascular bundle of the lesser gastric curvature in primary RYGB surgery if technically possible, as it reduces the number of serious adverse events after surgery. 


\section{Activities}

This results of multiple chapters thesis has been fundamental for the new study design on the effects of SADI vs RYGB as revisional procedure after failed sleeve gastrectomy. It will add valuable information to the draft of a model which may be helpful in easy decision making to choose the correct revisional procedure when indicated.

\section{Innovation}

This thesis has shown that VBG is inferior as primary bariatric procedure, supporting previous research. New insights are provided on pre-operative factors that predict long-term success after a bariatric procedure, in this case primary VBG.

This thesis encompasses a study to compare revisional RYGB after either primary AGB or primary sleeve gastrectomy. Furthermore, it includes the first study to prove the safe use of a fast track protocol in the more complicated revisional bariatric procedures and proves that preserving the neurovascular bundle significantly reduces postoperative complications after primary RYGB.

\section{The future}

The search for the perfect bariatric procedure is still ongoing, however the focus is slowly turned towards screening, selection of patients and the proper follow-up. With this search, new procedures are bound to be developed, which can cause short- and long-term complications, which in turn may require new treatment strategies.

Currently, the quest for finding the best revisional procedure after SG to achieve additional weight loss is still ongoing. This thesis has suggested a prospective, randomized controlled trial to investigate the effect of SADI after failed SG in the near future. SADI was suggested as a better alternative to achieve additional weight loss, however, present day practice has already caught up with the future. Nonetheless, future research on the SADI is necessary, as the first published results are very promising. ${ }^{12}$

The main aim for future research on long-term complications after failed bariatric procedures should be to provide consensus and hopefully even guidelines on how to treat these challenging complications. This will be challenging nonetheless, as patient characteristics will be very diverse.

Finally, the main aim for bariatric surgery in general may demand more focus on the screening of the patients and the perfect postoperative follow-up. 


\section{REFERENCES}

1. Angrisani L, Santonicola A, lovino P, Formisano G, Buchwald H, Scopinaro N. Bariatric Surgery Worldwide 2013. Obes Surg. 2015;25(10):1822-32.

2. Angrisani L, Santonicola A, lovino P, Vitiello A, Higa K, Himpens J, et al. IFSO Worldwide Survey 2016: Primary, Endoluminal, and Revisional Procedures. Obes Surg. 2018;28(12): 3783-94.

3. Angrisani L, Santonicola A, lovino P, Vitiello A, Zundel N, Buchwald H, et al. Bariatric Surgery and Endoluminal Procedures: IFSO Worldwide Survey 2014. Obes Surg. 2017;27(9):2279-89.

4. Buchwald H, Oien DM. Metabolic/bariatric surgery Worldwide 2008. Obes Surg. 2009; 19(12):1605-11.

5. Finks JF, Kole KL, Yenumula PR, English WJ, Krause KR, Carlin AM, et al. Predicting risk for serious complications with bariatric surgery: results from the Michigan Bariatric Surgery Collaborative. Ann Surg. 2011;254(4):633-40.

6. Stenberg E, Szabo E, Agren G, Naslund E, Boman L, Bylund A, et al. Early complications after laparoscopic gastric bypass surgery: results from the Scandinavian Obesity Surgery Registry. Ann Surg. 2014;260(6):1040-7.

7. Weiner RA, El-Sayes IA, Theodoridou S, Weiner SR, Scheffel O. Early post-operative complications: incidence, management, and impact on length of hospital stay. A retrospective comparison between laparoscopic gastric bypass and sleeve gastrectomy. Obes Surg. 2013; 23(12):2004-12.

8. Bransen J, Gilissen LP, van Rutte PW, Nienhuijs SW. Costs of Leaks and Bleeding After Sleeve Gastrectomies. Obes Surg. 2015;25(10):1767-71.

9. Borisenko O, Adam D, Funch-Jensen P, Ahmed AR, Zhang R, Colpan Z, et al. Bariatric Surgery can Lead to Net Cost Savings to Health Care Systems: Results from a Comprehensive European Decision Analytic Model. Obes Surg. 2015;25(9):1559-68.

10. Dogan K, Kraaij L, Aarts EO, Koehestanie P, Hammink E, van Laarhoven CJ, et al. Fast-track bariatric surgery improves perioperative care and logistics compared to conventional care. Obes Surg. 2015;25(1):28-35.

11. Geubbels N, Bruin SC, Acherman YI, van de Laar AW, Hoen MB, de Brauw LM. Fast track care for gastric bypass patients decreases length of stay without increasing complications in an unselected patient cohort. Obes Surg. 2014;24(3):390-6.

12. Sanchez-Pernaute A, Herrera MA, Perez-Aguirre ME, Talavera $P$, Cabrerizo $L$, Matia $P$, et al. Single anastomosis duodeno-ileal bypass with sleeve gastrectomy (SADI-S). One to three-year follow-up. Obes Surg. 2010;20(12):1720-6. 
Dankwoord 



\section{DANKWOORD}

Een promotieboekje schrijven gaat niet over een nachtje ijs. Daar gaat, of je dat nou wil of niet, veel tijd in zitten. Dat is niet alleen je eigen tijd, maar ook tijd van vele anderen! Er zijn veel mensen die direct en/of indirect een bijdrage hebben geleverd aan de totstandkoming van dit boekje en die wil ik graag bedanken.

\section{Dr. S.W. Nienhuijs}

Geachte copromotor, beste Simon, zonder andere mensen tekort te doen ben jij verreweg de belangrijkste persoon in mijn promotietraject geweest. Puur toevallig had je mijn CV uit een stapel getrokken, zo kwamen wij met elkaar in contact. Onze samenwerking begon augustus 2013 en ging van simpel databasewerk naar opzetten van een promotieonderzoek naar samenwerken in de kliniek. Jouw passie voor de wetenschap is bewonderenswaardig, maar ik heb vooral respect voor jouw vermogen om hier $110 \%$ voor te geven zonder dat je je andere taken als chirurg, opleider, vader, enz. daarmee tekort doet.

Enorm bedankt voor je betrokkenheid, je tomeloze inzet en je grote vertrouwen in mij als onderzoeker en later ook als arts-assistent!

\section{Prof. Dr. J.A.W. Teijink}

Geachte promotor, beste Joep. Net na mijn sollicitatie voor de opleiding in september 2017 raakten wij aan de praat over mijn boekje en kwam van het een het ander. Een vaatchirurg als promotor van een boekje over bariatrische chirurgie, daar had ik mijn geld in ieder geval niet op ingezet van tevoren. Gelukkig is de link tussen "Integrated Care" en bariatrische chirurgie groot. Mijn oprechte dank voor de energie die jij samen met Nicole erin hebt gestoken en voor je vertrouwen in mijn boekje.

Prof. dr. Bouvy, prof. dr. Jansen, prof. dr. Greve, prof. dr. Hazebroek en dr. van Dielen

Ik wil jullie, als leden van de beoordelingscommissie, bedanken voor jullie tijd en inzet die jullie in de kritische beoordeling van mijn proefschrift hebben gestoken en voor de deelname aan de verdediging.

\section{Maatschap Chirurgie en Intensive Care Catharina Ziekenhuis}

Dank voor de prettige samenwerking en begeleiding in de jaren die ik in het Catharina Ziekenhuis heb door morgen brengen. $\mathrm{lk}$ heb het genoegen gehad veel van jullie te mogen leren. 


\section{Bariatrische chirurgen Catharina Ziekenhuis}

Jullie wil ik apart bedanken: Frans Smulders, Jean-Paul de Zoete, Misha Luyer en Gust van Montfort. Jullie hebben allen een belangrijke bijdrage aan dit boekje als medeauteurs! Dank voor jullie kritische visie en opmerkingen bij de verschillende onderzoeken, ze hebben me enorm geholpen!

\section{Maatschap Chirurgie Viecuri Medisch Centrum}

Bedankt voor jullie inzet en zeer fijne samenwerking in het (ruime) jaar dat ik bij jullie als ANIOS heb mogen werken. Dank voor jullie vertrouwen in mij als artsassistent in aanloop naar de sollicitatieronde voor de opleiding. Mede door jullie reed ik dagelijks met veel plezier van Eindhoven naar Venlo!

\section{Maatschap Chirurgie Máxima Medisch Centrum}

Dank voor de leerzame eerste $1 \frac{1 / 2}{2}$ jaar van mijn opleiding. Jullie laagdrempeligheid, expertise en gezelligheid maken mijn opleiding een feest. Ik kijk ernaar uit om, na een uitstapje naar het MUMC, nog jaren samen te kunnen werken.

In het bijzonder dank aan de bariatrische chirurgen. Jullie betrokken mij al snel bij het lopende onderzoek in jullie kliniek en zijn altijd te porren voor een inhoudelijke discussie, ook over mijn boekje.

\section{Arts-assistenten Catharina Ziekenhuis, Viecuri, Máxima \& MUMC+}

Ik heb inmiddels de halve regio aan ziekenhuizen gehad, maar dat boekje was de rode draad door al deze ziekenhuizen. Ik wil alle assistenten enorm bedanken met wie ik over de afgelopen jaren in de bovengenoemde ziekenhuizen met veel plezier heb mogen werken.

\section{Obesitascentrum Catharina Ziekenhuis}

Aan de werksfeer heeft het zeker niet gelegen tijdens mijn onderzoek. Lieve mensen van het Obesitascentrum, wat zijn jullie fijne collega's om mee te werken en wat hebben jullie mij fijn opgevangen als broekie in 2013. Miranda, Trea, Claudette en Bianca. Dank voor al jullie inzet voor de poli, maar zeker ook voor mijn onderzoek met alle brieven en telefoontjes. Mohammed, Kim, Femke, Marieke A, Willem, Eva, en Bea, jullie hebben fulltime het allerbeste voor met iedere patiënt die het Obesitascentrum binnenloopt, bewonderenswaardig! Dat geldt ook voor alle diëtisten die zich iedere dag inzetten voor een gezond leven. Dan de vele psychologen dit het centrum rijk is, waarvan ik Gerbrand speciaal ga noemen. Dank voor het mogen gebruiken van jouw database en jouw kritische visie bij het artikel over psychologische factoren! 


\section{Onderzoekers Catharina Ziekenhuis}

Een gezellige werksfeer is cruciaal en daar hebben jullie maximaal aan bijgedragen. Thijs, held. Ook al lagen onze promotieonderwerpen ver uit elkaar, ik ben blij dat wij elkaar hebben gevonden in het stappen op vrijdagavond, eindeloos veel bijnamen, le Bask en het schrijven van een paar van mijn artikelen. Respect voor de manier waarop jij de publicaties er doorheen knalt. Pieter en Yannick, de vaatboys. Dank voor al jullie gezelligheid, collegiale visie en bijdrage aan alle niet-onderzoek gerelateerde activiteiten, zoals de uitjes naar de $\mathrm{AH}$, de bitterballen en de presentaties. Geert, zowel als onderzoeker als arts-assistent een goede collega. Jij hebt net als Thijs je boekje er doorheen geknald. Respect. P.S. Bedankt voor het meebrengen van die drone... Sjaak, mijn bariatriebuddy. Dank je voor jouw wetenschappelijke input en jouw onmisbare bijdrage als 'partner in crime' bij de bezoekjes aan al die verschillende congressen. Wenen gaat ons niet vergeten.

Bedankt Teus, Emmeline, Maikel, Emiel, Lindy, David, Saskia en alle andere onderzoekers (en semi-artsen).

Alle overige medeauteurs

Dankjewel voor al jullie inzet voor de verschillende artikelen, deze was onmisbaar. Dr. Gilissen, beste Lennard, bedankt dat ik een van jouw vergevorderde onderzoeken af heb mogen maken, ik ben trots op het resultaat.

\section{De Juppies}

Bedankt mannen. Jullie zijn als team zowel op het veld als buiten het veld een welkome afleiding naast al die medische zaken!

\section{Vrienden}

Dank jullie wel allemaal. Ik ga er een aantal noemen: Jeroen, Gijs, Luco en Huib. Dank voor al die fantastische stapavonden (tenminste, voor zover ik me ze nog herinner...) en de geweldige vakanties! Jeroen, jij speciaal bedankt dat een technisch ingenieur als jij als paranimf wil fungeren bij deze medische promotie, echt top maat! Huib, jij bedankt voor je creatieve geest die ervoor heeft gezorgd dat dit boekje er zo gelikt uit ziet!

\section{Schoonfamilie}

Lieve familie Mooren, wij kennen elkaar nu al bijna 13 jaar en ik kom nog steeds met enorm veel plezier bij jullie over de vloer. Dank voor jullie interesse in mijn onderzoek en werk de afgelopen jaren. 


\section{Familie}

Lieve mama, beste Peter. Dank voor al jullie interesse in mijn onderzoek de afgelopen jaren, dat werkte erg motiverend. Bedankt ik altijd op jullie kan rekenen en dat jullie altijd voor mij klaar staan, dankjewel! Lieve opa (en oma, ook al ben je niet meer onder ons), deze woorden gelden voor jullie net zoveel! Stefan, mijn kleine (maar toch niet zo kleine) broertje. Wat hebben wij af en toe elkaar het leven zuur gemaakt. Bedankt dat jij desondanks mijn paranimf wil zijn bij deze promotie.

Iris

Lieve Iris, mijn allerbeste maatje. Wat een avontuur weer hè! Het is zo fijn om thuis te komen en daar iemand te hebben waarop je blindelings kan vertrouwen. Dankjewel voor jouw steun door dik en dun en jouw kritische blik waar ik hem nodig heb. Het is goed dat jij me met beide benen op de grond houdt. Ik hou van je, dat er nog maar heel veel mooie avonturen mogen volgen! 
List of publications 



\section{LIST OF PUBLICATIONS}

Hannemann PF, van Wezenbeek MR, Kolkman KA, Twiss EL, Berghmans $\mathrm{CH}$, Dirven PA, et al. CT scan-evaluated outcome of pulsed electromagnetic fields in the treatment of acute scaphoid fractures: a randomised, multicentre, doubleblind, placebo-controlled trial. Bone Joint J. 2014;96-B(8):1070-6.

van Wezenbeek MR, Smulders JF, de Zoete JP, Luyer MD, van Montfort G, Nienhuijs SW. Long-Term Results of Primary Vertical Banded Gastroplasty. Obes Surg. 2015;25(8):1425-30.

van Wezenbeek MR, Pouwels S, Buise MP, Smulders FJ, Nienhuijs SW, van Montfort G. Conventional versus fast track anaesthesia in an unselected group of patients undergoing revisional bariatric surgery. International Journal of Surgery Open. 2015; Vol.1:22-27.

van Wezenbeek MR, de Milliano MM, Nienhuijs SW, Friederich P, Gilissen LP. A Specifically Designed Stent for Anastomotic Leaks after Bariatric Surgery: Experiences in a Tertiary Referral Hospital. Obes Surg. 2016;26(8):1875-80.

van Wezenbeek MR, Smulders FJ, de Zoete JP, Luyer MD, van Montfort G, Nienhuijs SW. Long-term results after revisions of failed primary vertical banded gastroplasty. World J Gastrointest Surg. 2016;8(3):238-45.

van Wezenbeek MR, van Oudheusden TR, Smulders JF, Nienhuijs SW, Luyer MD. Transection versus preservation of the neurovascular bundle of the lesser omentum in primary Roux-en-Y gastric bypass surgery. Surg Obes Relat Dis. 2016;12(2):283-9.

van Wezenbeek MR, van Hout GCM, Nienhuijs SW. Medical and Psychological Predictors for Long-Term Bariatric Success Using Primary Vertical-Banded Gastroplasty as a Model. Bariatric Surgical Practice and Patient Care. 2016;11(3): 110-115.

van Wezenbeek MR, Smulders FJ, Luyer MD, van Montfort G, Vanhimbeeck FJ, Nienhuijs SW. Predictors for the occurrence of major complications after primary Roux-en-Y Gastric Bypass surgery. Minerva Chir. 2016; 71(5):286-92.

van Wezenbeek MR, van Oudheusden TR, de Zoete JP, Smulders JF, Nienhuijs SW. Conversion to Gastric Bypass After Either Failed Gastric Band or Failed Sleeve Gastrectomy. Obes Surg. 2017;27(1):83-89. 
Ceha CMM, van Wezenbeek MR, Versteegden DPA, Smulders FJ, Nienhuijs SW. Matched Short-Term Results of SADI Versus GBP After Sleeve Gastrectomy. Obes Surg. 2018;28(12):3809-3814. 
Curriculum vitae 



\section{CURRICULUM VITAE}

Martin Richard van Wezenbeek was born on November 16, 1988, in Waalwijk, the Netherlands. He grew up in Sprang-Capelle in a family of 2 children. After graduating from high school (Gymnasium, Willem van Oranje College) in Waalwijk in 2007, he started his Medicine study at Maastricht University. After internships in several hospitals in the Netherlands and in Ayr, Scotland, he spent his final year at the trauma surgery department in Maastricht. When he received his medical degree in 2013, he started working at the bariatric surgery department in the Catharina Hospital in Eindhoven. From August 2013 until April 2015, he worked as a full time PhD candidate under the direct supervision of $d r$.

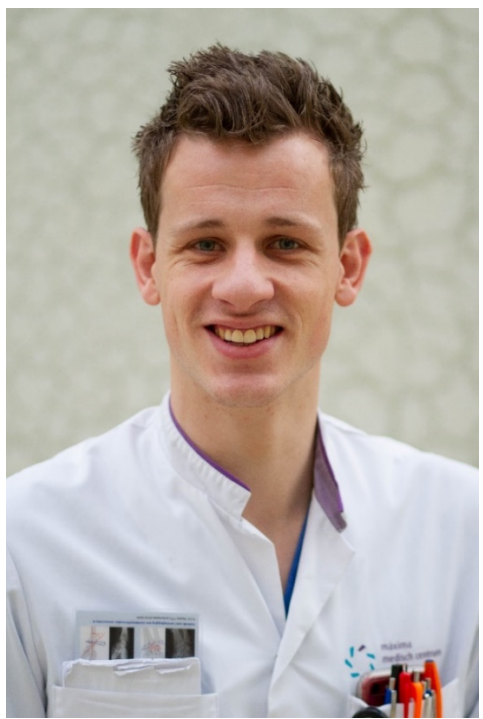
S.W. Nienhuijs, working on the current thesis and database management. From May 2015 until August 2015, he worked as a resident at the intensive care department, before transferring as a resident to the surgical department, still at the Catharina Hospital. In November 2016, he took on a new challenge as resident at the surgical department in the VieCuri Medical Center in Venlo.

January 2018, he started his specialization in general surgery at the Máxima Medical Center in Veldhoven and the Maastricht University Medical Center+, under the supervision of dr. R.M.H. Roumen and prof. dr. L.P.S. Stassen. During this period, he finished the current thesis. 
